\title{
Intermolecular C-O Bond Formation with Alkoxyl Radicals: Photoredox-Catalyzed $\alpha$-Alkoxylation of Carbonyl Compounds
}

Camille Banoun, Flavien Bourdreux, Emmanuel Magnier and Guillaume

Dagousset*

Université Paris-Saclay, UVSQ, CNRS, UMR 8180 Institut Lavoisier de Versailles, 78035

Versailles Cedex (France); E-mail: guillaume.dagousset@uvsq.fr

\section{Supporting Information}

\section{Table of contents}

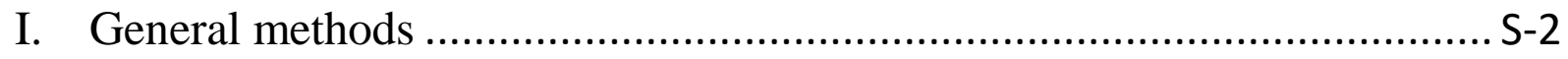

II. General procedures …...............................................................

III. Characterization of new compounds ……….................................. S-7

IV. Luminescence quenching experiments ......................................... S-27

V. Light On/Off experiment............................................................. S-28

VI. Quantum yield measurement.......................................................... S-29

VII. UV-Vis experiments ...................................................................... S-33

VIII. NMR spectra of new compounds .................................................... S-34

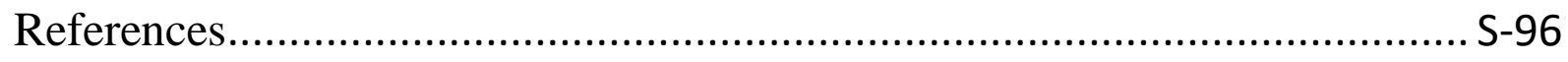




\section{General methods}

Each reaction was carried out under argon in a freshly distilled solvent, unless otherwise noted. All chemicals were purchased from Sigma-Aldrich, Fluorochem, TCI or Alfa Aesar and were used without further purification. Organic solvents were purchased from Sigma-Aldrich. $\mathrm{N}$ alkoxypyridinium salts were prepared following known procedure. ${ }^{[1]}$ Visible light irradiations were performed with a Flexled INSPIRE LED lamp $(3.45 \mathrm{~W} / \mathrm{m} ; \lambda=465 \mathrm{~nm})$. Reactions were monitored by thin-layer chromatography on silica gel $60 \mathrm{~F} 254$, or by ${ }^{19} \mathrm{~F}$ NMR spectroscopy. Unless otherwise noted, yields refer to materials purified by column chromatography. Flash chromatography was conducted on silica gel $60(40-63 \mu \mathrm{m})$ at medium pressure $(300 \mathrm{mbar}) .{ }^{1} \mathrm{H}$ and ${ }^{19} \mathrm{~F}$ NMR spectra were recorded with a Bruker AC-200 spectrometer. ${ }^{13} \mathrm{C}$ NMR spectra were recorded with a Bruker AC-300 spectrometer at $75 \mathrm{MHz}$ using a broadband decoupled mode with the multiplicities obtained using a DEPT sequence. NMR experiments were carried out in $\mathrm{CDCl}_{3}$, for which chemical shifts $(\delta)$ are reported in parts per million (ppm) with reference to $\mathrm{CHCl}_{3}\left({ }^{1} \mathrm{H}: 7.26 ;{ }^{13} \mathrm{C}: 77.07\right)$ and $\mathrm{CFCl}_{3}\left({ }^{19} \mathrm{~F}\right.$ : 0$)$. Coupling constants $(J)$ are reported in Hertz $(\mathrm{Hz})$. High-resolution electrospray mass spectra in the positive ion mode were obtained with a Xevo Q-Tof WATERS spectrometer.

\section{General procedures}

\section{GP 1: Synthesis of ketone-derived silylated enol ethers}

To a solution of ketone $(3 \mathrm{mmol})$ in dry $\mathrm{CH}_{2} \mathrm{Cl}_{2}(10 \mathrm{~mL})$ was added $\mathrm{Et}_{3} \mathrm{~N}$ (1.8 equiv) at RT under argon. Then, TIPSOTf (1.2 equiv) was slowly added, and the resulting solution was stirred at RT for $1 \mathrm{~h}$. A solution of $\mathrm{NaHCO}_{3}(5 \%, 20 \mathrm{~mL})$ was added, and extraction was carried out with $\mathrm{CH}_{2} \mathrm{Cl}_{2}(2 * 10 \mathrm{~mL})$. The organic phases were combined, dried over anhydrous $\mathrm{MgSO}_{4}$, and the solvents were evaporated under reduced pressure. The crude residue was then purified by flash chromatography $\left(\mathrm{SiO}_{2}\right.$ neutralized with $\left.\mathrm{Et}_{3} \mathrm{~N}\right)$ to afford the desired product 1a-1an. Spectroscopic data are in accordance with literature. ${ }^{[2]}$

\section{GP 2: Synthesis of silylketene $N, O$-acetals}

To a solution of amide ( $3 \mathrm{mmol}$ ) in dry THF (20 mL) was added LiHMDS (1M in THF, 1 equiv) dropwise at $-78^{\circ} \mathrm{C}$ under argon. After stirring $2 \mathrm{~h}$ at $-78^{\circ} \mathrm{C}$, TIPSOTf ( 1 equiv) was added, and stirring was continued for $2 \mathrm{~h}$ at $-78^{\circ} \mathrm{C}$. Then, the solvents were evaporated under reduced pressure. Et2O $(20 \mathrm{~mL})$ was added, and the solution was filtered on a pad of Celite. After evaporation of the solvents, the crude residue was then purified by flash chromatography $\left(\mathrm{SiO}_{2}\right.$ neutralized with $\mathrm{Et}_{3} \mathrm{~N}$ ) to afford the desired product 6a-6g. Spectroscopic data are in accordance with literature. ${ }^{[2]}$ 
GP 3: Photoredox-catalyzed $\alpha$-alkoxylation of silylated enol ethers and silylketene $N, O$ acetals

In a dry tube, under inert atmosphere, silylated enol ether 1a-1an or silylketene N,O-acetal 6a6g (0.20 mmol, 1 equiv.), $\mathrm{K}_{2} \mathrm{HPO}_{4}\left(69.7 \mathrm{mg}, 0.40 \mathrm{mmol}, 2\right.$ equiv.), $\mathrm{Ru}(\mathrm{bpy})_{3}\left(\mathrm{PF}_{6}\right)_{2} \mathbf{3 d}(1.7 \mathrm{mg}$, $0.002 \mathrm{mmol}, 1 \mathrm{~mol} \%)$, and $\mathrm{N}$-alkoxypyridinium salt 2 (0.36 mmol, 1.8 equiv.) are introduced. Then, $2 \mathrm{~mL}$ of dry MeCN are added and the solution is degazed under argon and stirred under blue LED irradiation $(7.5 \mathrm{~W}, 450 \mathrm{~nm})$ at room temperature during $18 \mathrm{~h}$. The crude mixture is purified on TLC preparative plate with a suitable eluent (pentane/ethyl acetate) to obtain the $\alpha$ alkoxylated desired product.

\section{GP 4: One-pot photoredox-catalyzed $\alpha$-alkoxylation of ketones}

In a dry tube, under inert atmosphere, a solution of ketone 5 ( $0.20 \mathrm{mmol}, 1$ equiv.) and 2,4,6collidine (1.5 equiv.) is prepared in $\mathrm{MeCN}(2 \mathrm{~mL}$ ) at $\mathrm{RT}$. Then TIPSOTf ( 1.2 equiv.) is added and the resulting solution is stirred $1 \mathrm{~h}$ at $\mathrm{RT}$. Then, $\mathrm{K}_{2} \mathrm{HPO}_{4}(69.7 \mathrm{mg}, 0.40 \mathrm{mmol}, 2$ equiv.), $\mathrm{Ru}(\mathrm{bpy})_{3}\left(\mathrm{PF}_{6}\right)_{2} \mathbf{3 d}(1.7 \mathrm{mg}, 0.002 \mathrm{mmol}, 1 \mathrm{~mol} \%)$, and $\mathrm{N}$-alkoxypyridinium salt 2 (0.40 mmol, 2 equiv.) are introduced. The solution is degazed with argon and stirred under blue LED irradiation $(7.5 \mathrm{~W}, 450 \mathrm{~nm})$ at room temperature during $18 \mathrm{~h}$. The crude mixture is purified on TLC preparative plate with a suitable eluent (pentane/ethyl acetate) to obtain the $\alpha$-alkoxylated desired product.

\section{Large-scale experiment procedure:}

In a dry 250mL flask, under inert atmosphere, silylated enol ether $\mathbf{1 a}(10 \mathrm{mmol}, 2.94 \mathrm{~g}, 1$ equiv) $\mathrm{K}_{2} \mathrm{HPO}_{4}$ (3.48 g, $20 \mathrm{mmol}, 2$ equiv.), Ru(bpy) ${ }_{3}\left(\mathrm{PF}_{6}\right)_{2} 3 \mathrm{~d}$ (86 mg, $0.1 \mathrm{mmol}, 1 \mathrm{~mol} \%$ ), and $\mathrm{N}$ methoxypyridinium salt 2a (4 g, $18 \mathrm{mmol}, 1.8$ equiv.) are introduced. Then, $100 \mathrm{~mL}$ of dry $\mathrm{MeCN}$ are added and the solution is degazed under argon and stirred under blue LED irradiation $(7.5 \mathrm{~W}, 450 \mathrm{~nm})$ at room temperature during $18 \mathrm{~h}$. Then the mixture is extracted with AtOAC and washed with water and brine. After evaporation of solvents, the crude mixture is purified on column chromatography with a pentane/ethyl acetate eluent to obtain the desired compound 4a $(1.48 \mathrm{~g}, 88 \%)$. 


\section{Synthesis of pyridinium salt 8.}

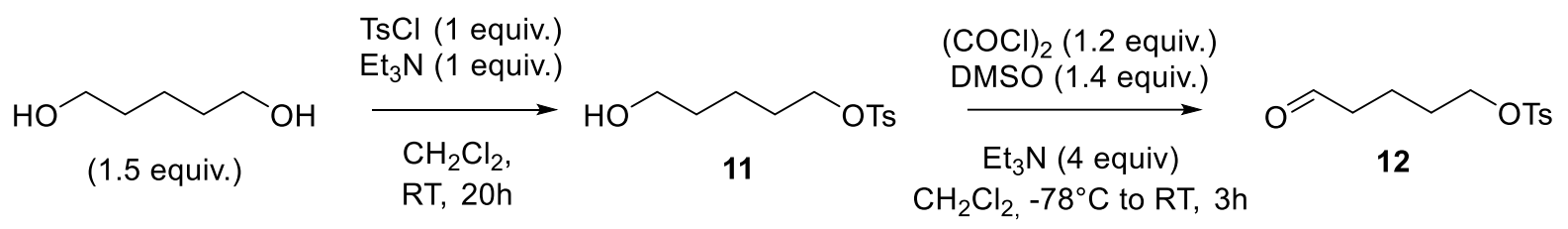

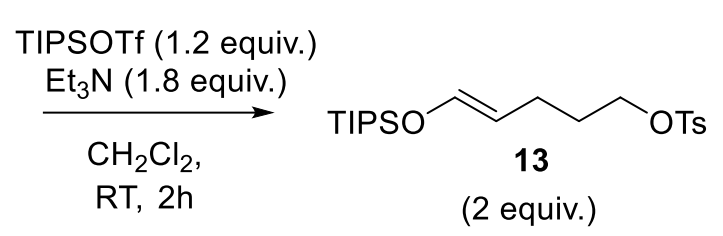

$\mathrm{RT}, 2 \mathrm{~h}$
(2 equiv.)

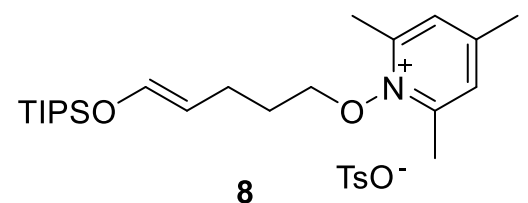

\section{5-hydroxypentyl 4-methylbenzenesulfonate 11}

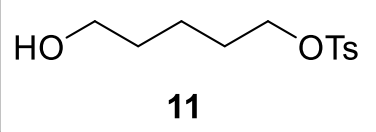

Procedure: to a mixture of 1,5-pentanediol ( $15.78 \mathrm{ml}, 0.15 \mathrm{~mol}, 1.5$ equiv.) and triethylamine (13.9 mL, $0.1 \mathrm{~mol}, 1$ equiv.) in $\mathrm{CH}_{2} \mathrm{Cl}_{2}(250 \mathrm{~mL}$ ) was added tosyl chloride (19.07 g, $0.1 \mathrm{~mol}, 1$ equiv.) slowly at $0^{\circ} \mathrm{C}$. The mixture was stirred at RT for $20 \mathrm{~h}$. The reaction was quenched with a saturated ammonium chloride solution at $0^{\circ} \mathrm{C}$ and extracted twice with DCM. The organic phase was dried over anhydrous magnesium sulfate, then filtered and concentrated under reduced pressure. The crude product was purified by a silica gel column chromatography using a mixture of pentane/ethyl acetate (50/50) as eluent to afford 11 (10.62g, 41\% yield) as a colorless oil. Spectroscopic data are in accordance with literature. ${ }^{[3]}$

${ }^{1}$ H NMR (200 MHz, CDCl3) $\delta 7.79(\mathrm{~d}, J=8.3 \mathrm{~Hz}, 2 \mathrm{H}), 7.35(\mathrm{~d}, J=8.0 \mathrm{~Hz}, 2 \mathrm{H}), 4.04(\mathrm{t}, J=$ $6.4 \mathrm{~Hz}, 2 \mathrm{H}), 3.61(\mathrm{t}, J=6.2 \mathrm{~Hz}, 2 \mathrm{H}), 2.46(\mathrm{~s}, 3 \mathrm{H}), 1.77-1.61(\mathrm{~m}, 2 \mathrm{H}), 1.58-1.33(\mathrm{~m}, 5 \mathrm{H})$.

\section{5-oxopentyl 4-methylbenzenesulfonate 12}

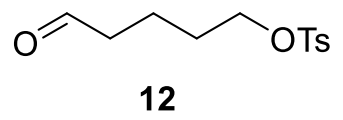


Procedure: A solution of DMSO (4.09 mL, $57.54 \mathrm{mmol}, 1.4$ equiv.) in $\mathrm{CH}_{2} \mathrm{Cl}_{2}$ (40 mL) was added dropwise in a two-neck round-bottom flask containing a solution of $(\mathrm{COCl})_{2}(4.23 \mathrm{~mL}$, $49.32 \mathrm{mmol}, 1.2$ equiv.) in $\mathrm{CH}_{2} \mathrm{Cl}_{2}(40 \mathrm{~mL})$ under atmosphere of Argon at $-78^{\circ} \mathrm{C}$ and stirred for $20 \mathrm{~min}$. Then a solution of 11 (10.62 g, $41.1 \mathrm{mmol}, 1$ equiv.) in $\mathrm{CH}_{2} \mathrm{Cl}_{2}$ (70 mL) was added dropwise, and the mixture was further stirred at $-78^{\circ} \mathrm{C}$ for $30 \mathrm{~min}$. Triethylamine $(22.9 \mathrm{~mL}$, $164.4 \mathrm{mmol}, 4$ equiv.) was added, and the reaction mixture was allowed to gradually warm to room temperature. The reaction mixture was then stirred for 1 additional hour, and quenched with a solution of $\mathrm{HCl} 1 \mathrm{M}(200 \mathrm{~mL})$. Water $(100 \mathrm{~mL})$ was then added and the reaction was extracted twice with $\mathrm{CH}_{2} \mathrm{Cl}_{2}$. The combined organic phases were washed with brine, dried over anhydrous magnesium sulfate, then filtered and concentrated under reduced pressure. The crude product was purified by a silica gel column chromatography using a mixture of pentane/ethyl acetate (70/30) as eluent to afford $\mathbf{1 2}(6.33 \mathrm{~g}, 60 \%$ yield) as a light yellow oil. Spectroscopic data are in accordance with literature. ${ }^{[4]}$

${ }^{1} \mathbf{H}$ NMR $\left(200 \mathrm{MHz}, \mathrm{CDCl}_{3}\right) \delta 9.73(\mathrm{t}, J=1.4 \mathrm{~Hz}, 1 \mathrm{H}), 7.84-7.73(\mathrm{~m}, 2 \mathrm{H}), 7.41-7.30(\mathrm{~m}, 2 \mathrm{H})$, $4.03(\mathrm{t}, J=4.2 \mathrm{~Hz}, 2 \mathrm{H}), 2.49-2.39(\mathrm{~m}, 5 \mathrm{H}), 1.68(\mathrm{dt}, J=4.7,2.5 \mathrm{~Hz}, 4 \mathrm{H})$.

\section{5-((triisopropylsilyl)oxy)pent-4-en-1-yl 4-methylbenzenesulfonate 13}

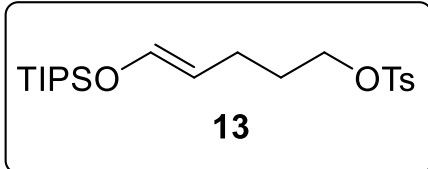

Procedure: To a solution of $\mathbf{1 2}$ (2 g, $7.8 \mathrm{mmol}, 1$ equiv.) and triethylamine (1.96 mL, 14.04 mmol, 1.8 equiv.) in $\mathrm{CH}_{2} \mathrm{Cl}_{2}(30 \mathrm{~mL})$ was added TIPSOTf $(2.52 \mathrm{~mL}, 9.36 \mathrm{mmol}, 1.2$ equiv.) slowly at RT. The reaction mixture was stirred at RT for $2 \mathrm{~h}$. The reaction was quenched with a solution of $\mathrm{NaHCO}_{3}$ sat. and extracted twice with $\mathrm{CH}_{2} \mathrm{Cl}_{2}$. The combined organic phases were dried over anhydrous magnesium sulfate, then filtered and concentrated under reduced pressure. The crude product was purified by a silica gel column chromatography using a mixture of pentane/ethyl acetate (95/5) and $1 \%$ of triethylamine as eluent to afford $\mathbf{1 3}$ (2.5g, $78 \%$ yield) as a colorless oil.

${ }^{1}$ H NMR $\left(300 \mathrm{MHz}, \mathrm{CD}_{3} \mathrm{CN}\right) \delta 7.78(\mathrm{~d}, J=8.0 \mathrm{~Hz}, 2 \mathrm{H}), 7.45(\mathrm{~d}, J=8.0 \mathrm{~Hz}, 2 \mathrm{H}), 6.34(\mathrm{~d}, J=$ $5.9 \mathrm{~Hz}, 1 \mathrm{H}), 4.35(\mathrm{dd}, J=13.6,6.8 \mathrm{~Hz}, 1 \mathrm{H}), 4.02(\mathrm{t}, J=6.7 \mathrm{~Hz}, 2 \mathrm{H}), 2.46(\mathrm{~s}, 3 \mathrm{H}), 2.08(\mathrm{q}, J=$ $7.3 \mathrm{~Hz}, 2 \mathrm{H}), 1.68$ (pent, $J=6.8 \mathrm{~Hz}, 2 \mathrm{H}), 1.16-1.04(\mathrm{~m}, 21 \mathrm{H})$.

${ }^{13}$ C NMR (75 MHz, CD 3 CN) $\delta 144.9,139.8,132.8,129.7,127.4,107.1,70.5,28.2,20.3,19.0$, $16.9,16.8$.

HRMS (ESI-TOF) m/z: [M+H]+ Calcd for $\mathrm{C}_{21} \mathrm{H}_{36} \mathrm{O}_{4} \mathrm{SSi} 413.2192$; Found 413.2182

\section{2,4,6-trimethyl-1-((5-((triisopropylsilyl)oxy)pent-4-en-1-yl)oxy)pyridin-1-ium tosylate 8}




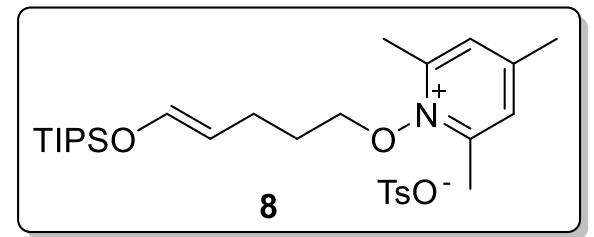

Procedure: To a solution of $\mathbf{1 3}$ (1.33 g, $3.22 \mathrm{mmol}, 2$ equiv.) in $\mathrm{MeCN}$ (10 mL) was added Collidine N-oxide (221 mg, $1.61 \mathrm{mmol}, 1$ equiv.). The reaction mixture was stirred at $80^{\circ} \mathrm{C}$ during $18 \mathrm{~h}$ thanks to an oil bath. The solvent was evaporated and the crude mixture was purified on TLC preparative plates using a mixture of $\mathrm{CH}_{2} \mathrm{Cl}_{2} / \mathrm{MeOH}(90 / 10)$ as eluent to afford $\mathbf{8}(135$ mg, $15 \%$ yield) as a colorless oil.

${ }^{1} \mathbf{H}$ NMR $\left(300 \mathrm{MHz}, \mathrm{CD}_{3} \mathrm{CN}\right) \delta 7.61(\mathrm{~d}, J=7.9 \mathrm{~Hz}, 2 \mathrm{H}), 7.58(\mathrm{~s}, 2 \mathrm{H}), 7.17(\mathrm{~d}, J=7.9 \mathrm{~Hz}, 2 \mathrm{H})$, $6.43(\mathrm{~d}, J=5.5 \mathrm{~Hz}, 1 \mathrm{H}), 4.52(\mathrm{q}, J=6.5 \mathrm{~Hz}, 1 \mathrm{H}), 4.34(\mathrm{t}, J=6.2 \mathrm{~Hz}, 2 \mathrm{H}), 2.74(\mathrm{~s}, 6 \mathrm{H}), 2.52$ (s, $3 \mathrm{H}), 2.37-2.20(\mathrm{~m}, 5 \mathrm{H}), 1.27-1.24(\mathrm{~m}, 2 \mathrm{H}), 1.16-1.04(\mathrm{~m}, 21 \mathrm{H})$.

${ }^{13}$ C NMR (75 MHz, CD 3 CN) $\delta 157.4,151.9,140.2,140.2,128.2,128.2,127.9,125.4,107.1$, 79.3, 29.6, 26.9, 20.3, 20.0, 19.1, 16.8, 16.4 .

HRMS (ESI-TOF) m/z: [M+H]+ Calcd for $\mathrm{C}_{22} \mathrm{H}_{40} \mathrm{NO}_{2} \mathrm{Si} 378.2823$; Found 378.2828. 


\section{Characterization of new compounds}

1-(4-Fluorophenyl)-2-methoxyethanone 4a

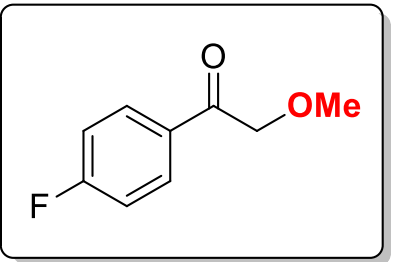

$\mathrm{m}=30 \mathrm{mg}, 89 \%$ yield, colorless oil, using pentane/EtOAc 85/15 as eluent.

${ }^{1} \mathbf{H}$ NMR (300 MHz, CDCl $\left.\mathbf{3}\right) \boldsymbol{\delta}$ (ppm): 7.98 (dd, 9.0 and $\left.5.6 \mathrm{~Hz}, 2 \mathrm{H}\right), 7.14$ (t, 8.4 Hz, 2H), 4.66 $(\mathrm{s}, 2 \mathrm{H}), 3.50(\mathrm{~s}, 3 \mathrm{H})$.

${ }^{13}$ C NMR (75 MHz, CDCl 3 ) $\boldsymbol{\delta}$ (ppm): 194.8, 166.0 (d, 255.8 Hz), 131.3 (d, 2.7 Hz), 130.7 (d, $9.3 \mathrm{~Hz}), 115.9$ (d, 22.0 Hz), 75.4, 59.5.

${ }^{19}$ F NMR (282 MHz, CDCl 3 ) $\delta$ (ppm): -104.05.

HRMS (ESI-TOF) m/z: [M-MeOH+H]+ Calcd for $\mathrm{C}_{8} \mathrm{H}_{6} \mathrm{OF}$ 137.0403; Found 137.0399.

2-Methoxy-1-(4-methoxyphenyl)ethanone 4b

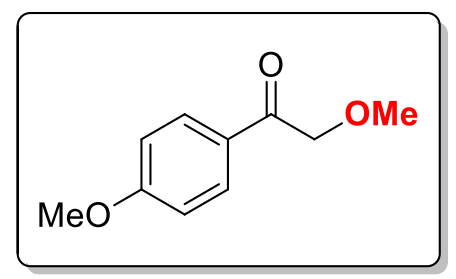

$\mathrm{m}=26 \mathrm{mg}, 72 \%$ yield, white solid, using pentane/EtOAc 80/20 as eluent.

Melting point: $42-44^{\circ} \mathrm{C}$

${ }^{1}$ H NMR (300 MHz, CDCl $)$ 反 (ppm): $7.91(\mathrm{~d}, 8.7 \mathrm{~Hz}, 2 \mathrm{H}), 6.92(\mathrm{~d}, 8.7 \mathrm{~Hz}, 2 \mathrm{H}), 4.64(\mathrm{~s}, 2 \mathrm{H})$, $3.86(\mathrm{~s}, 3 \mathrm{H}), 3.49$ (s, 3H).

${ }^{13}$ C NMR (75 MHz, CDCl 3 ) $\delta$ (ppm): 194.7, 163.8, 130.2, 127.9, 113.9, 75.2, 59.4, 55.5.

HRMS (ESI-TOF) m/z: [M+H]+Calcd for $\mathrm{C}_{10} \mathrm{H}_{13} \mathrm{O}_{3}$ 181.0865; Found 181.0862.

\section{2-Methoxy-1-(p-tolyl)ethanone 4c}

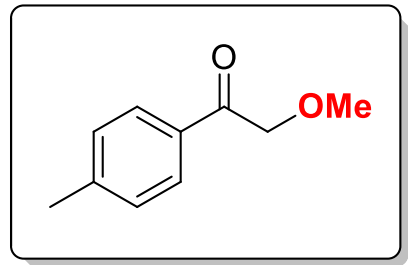

$\mathrm{m}=28 \mathrm{mg}, 85 \%$ yield, colorless oil, using pentane/EtOAc 80/20 as eluent.

${ }^{1} \mathbf{H}$ NMR (300 MHz, CDCl$) \boldsymbol{\delta}$ (ppm): $7.81(\mathrm{~d}, 8.2 \mathrm{~Hz}, 2 \mathrm{H}), 7.25(\mathrm{~d}, 8.2 \mathrm{~Hz}, 2 \mathrm{H}), 4.67$ (s, 2H), $3.49(\mathrm{~s}, 3 \mathrm{H}), 2.40(\mathrm{~s}, 3 \mathrm{H})$. 
${ }^{13}$ C NMR (75 MHz, CDCl 3 ) $\delta$ (ppm): 195.8, 144.5, 132.4, 129.4, 127.9, 75.2, 59.5, 21.8.

HRMS (ESI-TOF) m/z: [M+Na]+ Calcd for $\mathrm{C}_{10} \mathrm{H}_{12} \mathrm{O}_{2} \mathrm{Na}$ 187.0735; Found 187.0736.

\section{1-(4-Chlorophenyl)-2-methoxyethanone 4d}

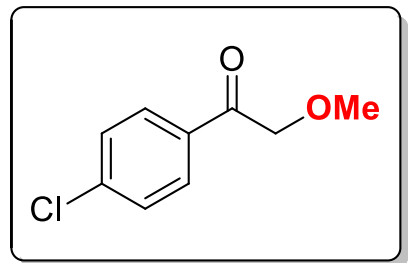

$\mathrm{m}=30 \mathrm{mg}, 81 \%$ yield, white solid, using pentane/EtOAc 80/20 as eluent.

Melting point: $62-64^{\circ} \mathrm{C}$

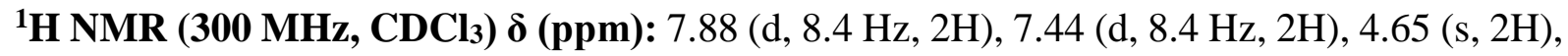
$3.49(\mathrm{~s}, 3 \mathrm{H})$.

${ }^{13}$ C NMR (75 MHz, CDCl3) $\delta$ (ppm): 195.2, 140.1, 133.1, 129.4, 129.1, 75.4, 59.5.

HRMS (ESI-TOF) m/z: [M-MeOH+H]+ Calcd for $\mathrm{C}_{8} \mathrm{H}_{6} \mathrm{OCl}$ 153.0107; Found 153.0107.

1-(4-Bromophenyl)-2-methoxyethanone 4e

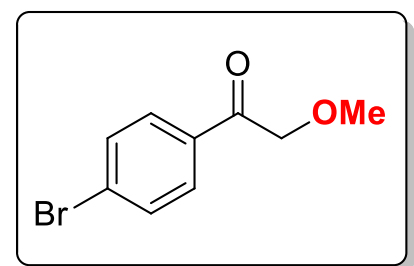

$\mathrm{m}=38 \mathrm{mg}, 83 \%$ yield, white solid, using pentane/EtOAc $80 / 20$ as eluent.

Melting point: $72-74^{\circ} \mathrm{C}$

${ }^{1}$ H NMR (300 MHz, CDCl3) $\boldsymbol{\delta}$ (ppm): $7.80(\mathrm{~d}, 8.4 \mathrm{~Hz}, 2 \mathrm{H}), 7.60$ (d, 8.4 Hz, 2H), 4.64 (s, 2H), $3.48(\mathrm{~s}, 3 \mathrm{H})$.

${ }^{13}$ C NMR (75 MHz, CDCl $)$ ) (ppm): 195.4, 133.5, 132.1, 129.5, 128.8, 75.3, 59.5.

HRMS (ESI-TOF) m/z: [M-MeOH+H]+Calcd for $\mathrm{C}_{8} \mathrm{H}_{6} \mathrm{OBr}$ 196.9602; Found 196.9597.

\section{2-Methoxy-1-(o-tolyl)ethanone 4f}

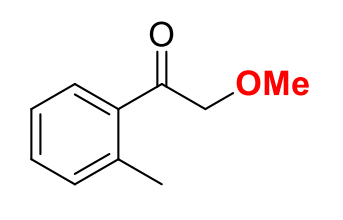

$\mathrm{m}=25 \mathrm{mg}, 76 \%$ yield, colorless oil, using pentane/EtOAc 80/20 as eluent.

${ }^{1} \mathbf{H}$ NMR (300 MHz, CDCl 3$) \boldsymbol{\delta}$ (ppm): 7.59 (d, $\left.7.7 \mathrm{~Hz}, 1 \mathrm{H}\right), 7.43$ (t, $\left.7.7 \mathrm{~Hz}, 1 \mathrm{H}\right)$, 7.31-7.26 $(\mathrm{m}, 2 \mathrm{H}), 4.59(\mathrm{~s}, 2 \mathrm{H}), 3.52(\mathrm{~s}, 3 \mathrm{H}), 2.55(\mathrm{~s}, 3 \mathrm{H})$. 
${ }^{13}$ C NMR (75 MHz, CDCl $)$ ) (ppm): 200.3, 138.6, 135.3, 132.1, 131.8, 128.2, 125.7, 76.7, 59.4, 21.1.

HRMS (ESI-TOF) m/z: [M-MeOH+H]+ Calcd for $\mathrm{C}_{9} \mathrm{H}_{9} \mathrm{O}$ 133.0653; Found 133.0653.

\section{2-Methoxy-1-phenylethanone 4g}

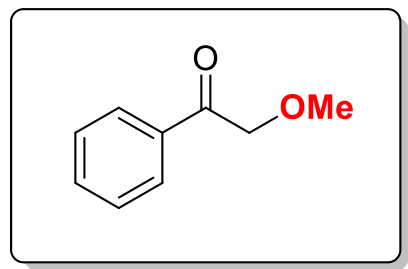

$\mathrm{m}=23 \mathrm{mg}, 77 \%$ yield, colorless oil, using pentane/EtOAc 80/20 as eluent.

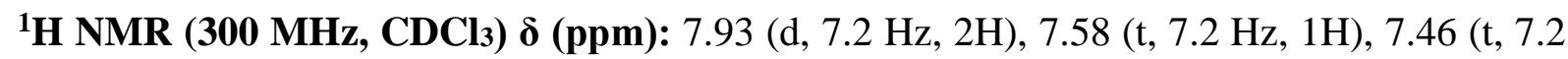
$\mathrm{Hz}, 2 \mathrm{H}), 4.71$ (s, 2H), 3.51 (s, 3H).

${ }^{13}$ C NMR (75 MHz, CDCl3) $\delta$ (ppm): 196.2, 134.9, 133.6, 128.8, 127.9, 75.3, 59.5.

HRMS (ESI-TOF) m/z: [M+Na]+ Calcd for $\mathrm{C}_{9} \mathrm{H}_{10} \mathrm{O}_{2} \mathrm{Na}$ 173.0578; Found 173.0582.

Methyl 3-(2-methoxyacetyl)benzoate 4h

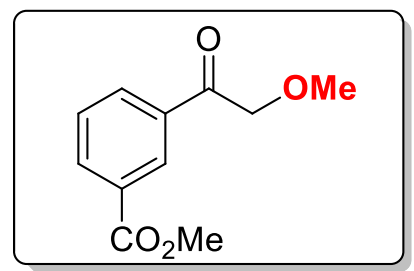

$\mathrm{m}=37 \mathrm{mg}, 89 \%$ white solid, using pentane/EtOAc 75/25 as eluent.

Melting point: $55-57^{\circ} \mathrm{C}$

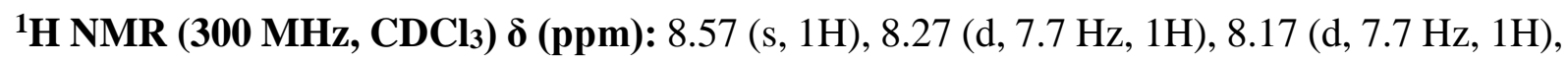
7.59 (t, $7.7 \mathrm{~Hz}, 2 \mathrm{H}), 4.77$ (s, 2H), 3.98 (s, 3H), 3.55 (s, 3H).

${ }^{13}$ C NMR (75 MHz, CDCl 3 ) $\delta$ (ppm): 195.4, 166.1, 135.0, 134.3, 132.1, 130.8, 129.1, 128.9, 75.4, 59.5, 52.5 .

HRMS (ESI-TOF) m/z: [M+H]+Calcd for $\mathrm{C}_{11} \mathrm{H}_{13} \mathrm{O}_{4}$ 209.0814; Found 209.0810.

2-Methoxy-1-(3-(trifluoromethyl)phenyl)ethanone 4i

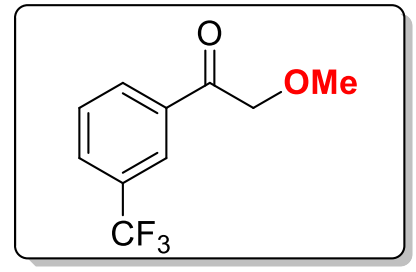

$\mathrm{m}=31 \mathrm{mg}, 71 \%$ yield, yield, colorless oil, using pentane/EtOAc 80/20 as eluent. 
${ }^{1} \mathbf{H}$ NMR (300 MHz, CDCl 3$) \boldsymbol{\delta}(\mathbf{p p m}): 8.13(\mathrm{~s}, 1 \mathrm{H}), 8.07(\mathrm{~d}, 7.7 \mathrm{~Hz}, 1 \mathrm{H}), 7.77(\mathrm{~d}, 7.7 \mathrm{~Hz}, 1 \mathrm{H})$, $7.56(\mathrm{t}, 7.7 \mathrm{~Hz}, 2 \mathrm{H}), 4.64$ (s, 2H), 3.45 (s, 3H).

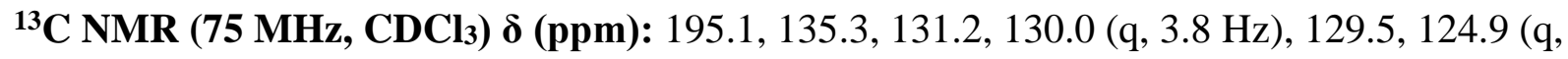
$3.8 \mathrm{~Hz}), 123.6$ (q, 272.3 Hz), 75.5, 59.6.

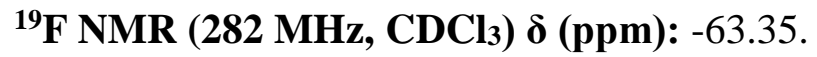

HRMS (ESI-TOF) m/z: [M+Na]+ Calcd for $\mathrm{C}_{10} \mathrm{H}_{9} \mathrm{O}_{2} \mathrm{~F}_{3} \mathrm{Na}$ 241.0452; Found 241.0460.

\section{3-(2-Methoxyacetyl)benzonitrile $4 \mathbf{j}$}

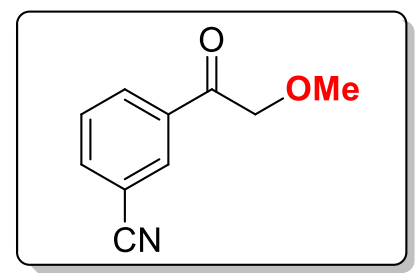

$\mathrm{m}=20 \mathrm{mg}, 57 \%$ yield, white solid, using pentane/EtOAc 75/25 as eluent.

Melting point: $103-105^{\circ} \mathrm{C}$

${ }^{1} \mathbf{H}$ NMR (300 MHz, CDCl 3$) \boldsymbol{\delta}$ (ppm): 8.24 (s, 1H), $8.18(\mathrm{~d}, 7.9 \mathrm{~Hz}, 1 \mathrm{H}), 7.86(\mathrm{~d}, 7.9 \mathrm{~Hz}, 1 \mathrm{H})$, $7.62(\mathrm{t}, 7.9 \mathrm{~Hz}, 2 \mathrm{H}), 4.66$ (s, 2H), $3.50(\mathrm{~s}, 3 \mathrm{H})$.

${ }^{13}$ C NMR (75 MHz, CDCl 3 ) $\boldsymbol{\delta}$ (ppm): 194.6, 136.4, 135.6, 132.1, 131.9, 129.8, 117.8, 113.3, 75.6, 59.6 .

HRMS (ESI-TOF) m/z: [M+Na]+ Calcd for $\mathrm{C}_{10} \mathrm{H}_{9} \mathrm{NO}_{2} \mathrm{Na}$ 198.0525; Found 198.0520.

Tert-butyl 3-(2-methoxyacetyl)-1H-indole-1-carboxylate 4k

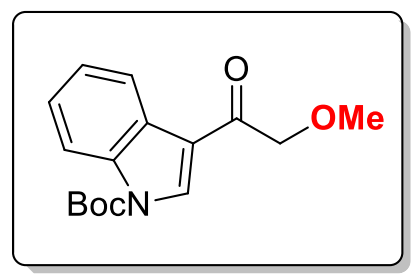

$\mathrm{m}=20 \mathrm{mg}, 35 \%$ yield, white solid, using pentane/EtOAc 80/20 as eluent.

Melting point: $148-150^{\circ} \mathrm{C}$

${ }^{1} \mathbf{H}$ NMR (300 MHz, CDCl 3 ) $\boldsymbol{\delta}$ (ppm): 8.50 (s, 1H), 8.41 (dd, 5.9 and $\left.2.3 \mathrm{~Hz}, 1 \mathrm{H}\right), 8.17$ (dd, 5.9 and $2.3 \mathrm{~Hz}, 1 \mathrm{H}), 7.44-7.40(\mathrm{~m}, 2 \mathrm{H}), 4.52(\mathrm{~s}, 2 \mathrm{H}), 3.52(\mathrm{~s}, 3 \mathrm{H}), 1.71(\mathrm{~s}, 9 \mathrm{H})$.

${ }^{13}$ C NMR (75 MHz, CDCl 3 ) $\boldsymbol{\delta}$ (ppm): 193.3, 149.1, 135.2, 127.6, 125.6, 124.5, 122.6, 117.5, 115.0, 85.6, 77.3, 59.5, 28.1 .

HRMS (ESI-TOF) m/z: [M+Na]+Calcd for $\mathrm{C}_{16} \mathrm{H}_{19} \mathrm{NO}_{4} \mathrm{Na} 312.1212$; Found 312.1208.

1-(Benzofuran-2-yl)-2-methoxyethanone 4l 


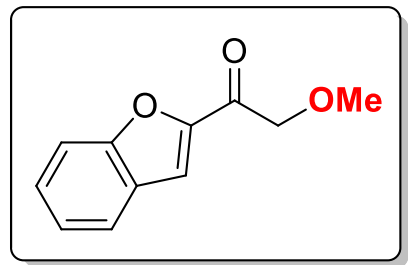

$\mathrm{m}=32 \mathrm{mg}, 84 \%$ yield, white solid, using pentane/EtOAc 80/20 as eluent.

Melting point: $80-82^{\circ} \mathrm{C}$

${ }^{1} \mathbf{H}$ NMR (300 MHz, CDCl 3$)$ 反 (ppm): $7.71(\mathrm{~d}, 7.9 \mathrm{~Hz}, 1 \mathrm{H}), 7.65(\mathrm{~s}, 1 \mathrm{H}), 7.57(\mathrm{~d}, 8.4 \mathrm{~Hz}, 1 \mathrm{H})$, $7.48(\mathrm{td}, 8.2$ and $1.0 \mathrm{~Hz}, 1 \mathrm{H}), 7.32(\mathrm{t}, 7.9 \mathrm{~Hz}, 1 \mathrm{H}), 4.67(\mathrm{~s}, 2 \mathrm{H}), 3.54(\mathrm{~s}, 3 \mathrm{H})$.

${ }^{13}$ C NMR (75 MHz, CDCl 3 ) $\delta$ (ppm): 187.7, 155.5, 150.7, 128.6, 126.8, 124.1, 123.5, 113.8, $112.5,75.4,59.7$.

HRMS (ESI-TOF) m/z: [M+H]+Calcd for $\mathrm{C}_{11} \mathrm{H}_{11} \mathrm{O}_{3}$ 191.0708; Found 191.0705.

\section{1-(Benzo[b]thiophen-2-yl)-2-methoxyethanone 4m}

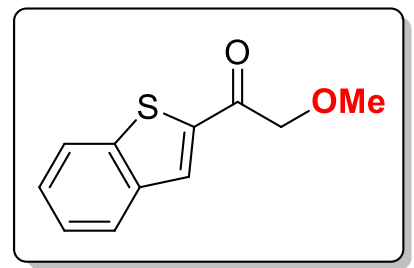

$\mathrm{m}=24 \mathrm{mg}, 58 \%$ yield, white solid, using pentane/EtOAc 85/15 as eluent.

Melting point: $90-92^{\circ} \mathrm{C}$

${ }^{1} \mathbf{H}$ NMR (300 MHz, CDCl 3 ) $\boldsymbol{\delta}$ (ppm): 8.11 (s, 1H), 7.91-7.86 (m, 2H), 7.51-7.38 (m, 2H), $4.65(\mathrm{~s}, 2 \mathrm{H}), 3.53(\mathrm{~s}, 3 \mathrm{H})$.

${ }^{13}$ C NMR (75 MHz, CDCl3) $\boldsymbol{\delta}$ (ppm): 191.4, 142.4, 140.5, 139.0, 129.8, 127.7, 126.1, 125.2, $123.0,75.9,59.7$.

HRMS (ESI-TOF) m/z: [M+H]+ Calcd for $\mathrm{C}_{11} \mathrm{H}_{11} \mathrm{O}_{2} \mathrm{~S}$ 207.0480; Found 207.0478.

\section{1-(4-Chlorophenyl)-2-methoxypropan-1-one 4n}

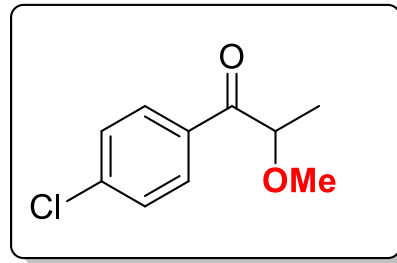

$\mathrm{m}=34 \mathrm{mg}, 86 \%$ yield, white solid, using pentane/EtOAc 85/15 as eluent.

Melting point: $64-66^{\circ} \mathrm{C}$

${ }^{1} \mathbf{H}$ NMR (300 MHz, CDCl $)$ $\boldsymbol{\delta}$ (ppm): $8.01(\mathrm{~d}, 8.4 \mathrm{~Hz}, 2 \mathrm{H}), 7.43(\mathrm{~d}, 8.4 \mathrm{~Hz}, 2 \mathrm{H}), 4.52$ (q, 6.9 $\mathrm{Hz}, 1 \mathrm{H}), 3.36$ (s, 3H), 1.47 (d, $6.9 \mathrm{~Hz}, 3 \mathrm{H})$.

${ }^{13}$ C NMR (75 MHz, CDCl 3 ) $\delta$ (ppm): 195.6, 139.9, 133.0, 130.3, 129.0, 80.7, 57.4, 18.4. 
HRMS (ESI-TOF) m/z: [M+Na]+Calcd for $\mathrm{C}_{10} \mathrm{H}_{11} \mathrm{O}_{2} \mathrm{ClNa} 221.0345$; Found 221.0340.

2-Methoxy-2-methyl-1-phenylpropan-1-one 4o

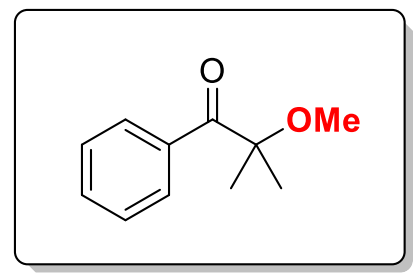

$\mathrm{m}=28 \mathrm{mg}, 79 \%$ yield, colorless oil, using pentane/EtOAc 90/10 as eluent.

${ }^{1}$ H NMR (300 MHz, CDCl3) $\boldsymbol{\delta}$ (ppm): 8.27 (d, 7.4 Hz, 2H), 7.54 (t, 7.4 Hz, 1H), 7.43 (t, 7.4 $\mathrm{Hz}, 2 \mathrm{H}), 3.19$ (s, 3H), 1.52 (s, 6H).

${ }^{13}$ C NMR (75 MHz, CDCl 3 ) $\boldsymbol{\delta}$ (ppm): 203.7, 134.9, 132.9, 129.8, 128.4, 83.4, 52.5, 24.8.

HRMS (ESI-TOF) m/z: [M+Na]+ Calcd for $\mathrm{C}_{10} \mathrm{H}_{14} \mathrm{O}_{2} \mathrm{Na} 201.0882$; Found 201.0886.

\section{2-Methoxy-3,4-dihydronaphthalen-1(2H)-one $4 p$}

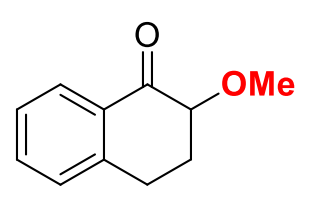

$\mathrm{m}=29 \mathrm{mg}, 82 \%$ yield, white solid, using pentane/EtOAc 80/20 as eluent.

Melting point: $34-36^{\circ} \mathrm{C}$

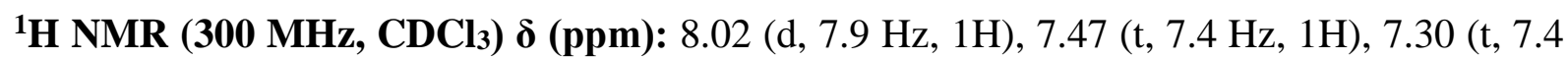
$\mathrm{Hz}, 1 \mathrm{H}), 7.23(\mathrm{~d}, 7.7 \mathrm{~Hz}, 1 \mathrm{H}), 3.94(\mathrm{dd}, 10.8$ and $3.1 \mathrm{~Hz}, 1 \mathrm{H}), 3.57(\mathrm{~s}, 3 \mathrm{H}), 3.18-2.96(\mathrm{~m}, 2 \mathrm{H})$, 2.43-2.34 (m, 1H), 2.25-2.12 (m, 1H).

${ }^{13}$ C NMR (75 MHz, CDCl3) $\delta$ (ppm): 196.6, 143.4, 133.6, 131.9, 128.6, 127.7, 126.8, 81.7, 58.2, 29.5, 27.2 .

HRMS (ESI-TOF) m/z: [M+Na]+Calcd for $\mathrm{C}_{11} \mathrm{H}_{12} \mathrm{O}_{2} \mathrm{Na}$ 199.0735; Found 199.0731.

\section{3-Methoxychroman-4-one 4q}

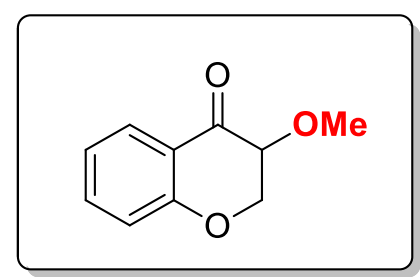

$\mathrm{m}=19 \mathrm{mg}, 53 \%$ yield, white solid, using pentane/EtOAc 80/20 as eluent.

Melting point: $47-48^{\circ} \mathrm{C}$ 
${ }^{1} \mathbf{H}$ NMR (300 MHz, CDCl 3 ) $\boldsymbol{\delta}$ (ppm): 7.89 (dd, 7.7 and $\left.1.5 \mathrm{~Hz}, 1 \mathrm{H}\right), 7.49$ (ddd, 8.4 and 7.4 and $1.5 \mathrm{~Hz}, 1 \mathrm{H}), 7.04(\mathrm{dd}, 7.7$ and $7.4 \mathrm{~Hz}, 1 \mathrm{H}), 6.97(\mathrm{~d}, 8.4 \mathrm{~Hz}, 1 \mathrm{H}), 4.54-4.43(\mathrm{~m}, 2 \mathrm{H}), 3.93$ (dd, 6.7 and $4.6 \mathrm{~Hz}, 1 \mathrm{H}), 3.58$ (s, 3H).

${ }^{13}$ C NMR (75 MHz, CDCl3) $\delta$ (ppm): 190.5, 161.1, 136.2, 127.6, 121.8, 119.8, 117.8, 77.3, 69.6, 59.0.

HRMS (ESI-TOF) m/z: [M+H]+ Calcd for $\mathrm{C}_{10} \mathrm{H}_{11} \mathrm{O}_{3}$ 179.0708; Found 179.0709.

\section{6-Methoxy-6,7,8,9-tetrahydro-5H-benzo[7]annulen-5-one 4r}

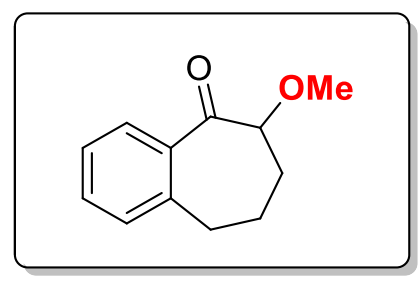

$\mathrm{m}=28 \mathrm{mg}, 74 \%$ yield, white solid, using pentane/EtOAc 90/10 as eluent.

Melting point: $84-86^{\circ} \mathrm{C}$

${ }^{1} \mathbf{H}$ NMR (300 MHz, CDCl 3$)$ 反 (ppm): 7.70 (dd, 7.7 and $\left.1.3 \mathrm{~Hz}, 1 \mathrm{H}\right), 7.39$ (td, 7.4 and $1.3 \mathrm{~Hz}$, $1 \mathrm{H}), 7.31(\mathrm{~d}, 7.4 \mathrm{~Hz}, 1 \mathrm{H}), 7.21(\mathrm{~d}, 7.4 \mathrm{~Hz}, 1 \mathrm{H}), 4.16(\mathrm{dd}, 7.7$ and $5.9 \mathrm{~Hz}, 1 \mathrm{H}), 3.32(\mathrm{~s}, 3 \mathrm{H})$, 3.11-2.89 (m, 2H), 2.25-2.15 (m, 1H), 2.05-1.80 (m, 3H).

${ }^{13}$ C NMR (75 MHz, CDCl3) $\delta$ (ppm): 204.6, 142.3, 137.7, 131.7, 130.0, 128.7, 126.5, 86.1, 57.8, 34.7, 31.2, 23.7.

HRMS (ESI-TOF) m/z: [M+Na]+ Calcd for $\mathrm{C}_{12} \mathrm{H}_{14} \mathrm{O}_{2} \mathrm{Na} 213.0891$; Found 213.0883.

\section{2-Methoxy-2,3-dihydro-1H-inden-1-one 4s}

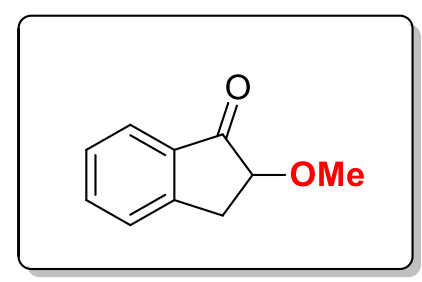

$\mathrm{m}=27 \mathrm{mg}, 83 \%$ yield white solid, using pentane/EtOAc 80/20 as eluent.

Melting point: $48-50^{\circ} \mathrm{C}$

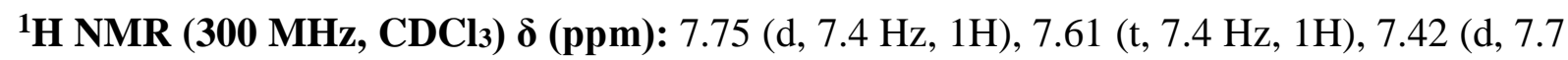
$\mathrm{Hz}, 1 \mathrm{H}), 7.37(\mathrm{t}, 7.4 \mathrm{~Hz}, 1 \mathrm{H}), 4.18(\mathrm{dd}, 7.4$ and $4.6 \mathrm{~Hz}, 1 \mathrm{H}), 3.63(\mathrm{~s}, 3 \mathrm{H}), 3.50$ (dd, 16.6 and $7.4 \mathrm{~Hz}, 1 \mathrm{H}), 3.00$ (dd, 16.6 and $4.6 \mathrm{~Hz}, 1 \mathrm{H})$.

${ }^{13}$ C NMR (75 MHz, CDCl $)$ ) (ppm): 203.9, 150.6, 135.7, 134.9, 128.0, 126.7, 124.4, 81.4, $58.3,33.3$.

HRMS (ESI-TOF) m/z: [M+Na]+ Calcd for $\mathrm{C}_{10} \mathrm{H}_{10} \mathrm{O}_{2} \mathrm{Na}$ 185.0578; Found 185.0578. 
Tert-butyl 2-methoxy-1-oxo-2,3-dihydro-1H-indene-2-carboxylate $4 \mathrm{t}$

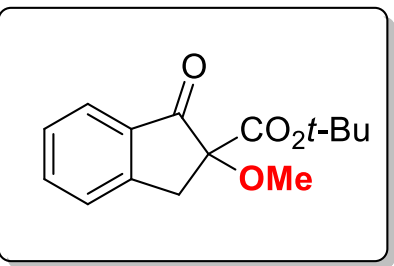

$\mathrm{m}=35 \mathrm{mg}, 67 \%$ yield, white solid, using pentane/EtOAc 80/20 as eluent.

Melting point: $54-56^{\circ} \mathrm{C}$

${ }^{1} \mathbf{H}$ NMR (300 MHz, CDCl 3 ) $\boldsymbol{\delta}$ (ppm): $7.78(\mathrm{~d}, 7.7 \mathrm{~Hz}, 1 \mathrm{H}), 7.62(\mathrm{t}, 7.4 \mathrm{~Hz}, 1 \mathrm{H}), 7.45(\mathrm{~d}, 7.7$ $\mathrm{Hz}, 1 \mathrm{H}), 7.40$ (t, $7.4 \mathrm{~Hz}, 1 \mathrm{H}), 3.58(\mathrm{~d}, 16.9 \mathrm{~Hz}, 1 \mathrm{H}), 3.53$ (s, 3H), 3.26 (d, $16.6 \mathrm{~Hz}, 1 \mathrm{H}), 1.40$ (s, 9H).

${ }^{13}$ C NMR (75 MHz, CDCl 3$) \boldsymbol{\delta}$ (ppm): 199.1, 168.6, 151.8, 135.8, 134.6, 128.0, 126.4, 125.0, 85.7, 83.0, 54.3, 38.3, 27.9.

HRMS (ESI-TOF) m/z: [M+Na]+ Calcd for $\mathrm{C}_{15} \mathrm{H}_{18} \mathrm{O}_{4} \mathrm{Na} 285.1103$; Found 285.1104.

\section{1-Methoxy-4-phenylbut-3-en-2-one 4u}<smiles>COCC(=O)/C=C/c1ccccc1</smiles>

$\mathrm{m}=22 \mathrm{mg}, 63 \%$ yield, white solid, using pentane/EtOAc 80/20 as eluent.

Melting point: $54-56^{\circ} \mathrm{C}$

${ }^{1}$ H NMR (300 MHz, CDCl3) $\boldsymbol{\delta}$ (ppm): 7.70 (d, $\left.16.1 \mathrm{~Hz}, 1 \mathrm{H}\right), 7.60-7.54$ (m, 2H), 7.41-7.38 (m, $3 \mathrm{H}), 6.94$ (d, $16.1 \mathrm{~Hz}, 1 \mathrm{H}), 4.25$ (s, 2H), 3.48 (s, 3H).

${ }^{13}$ C NMR (75 MHz, CDCl $)$ ) (ppm): 197.2, 143.7, 134.4, 130.8, 129.0, 128.6, 121.5, 77.3, 59.4.

HRMS (ESI-TOF) m/z: [M+Na]+ Calcd for $\mathrm{C}_{11} \mathrm{H}_{12} \mathrm{O}_{2} \mathrm{Na}$ 199.0735; Found 199.0741.

\section{1-Methoxynon-3-en-2-one 4v}

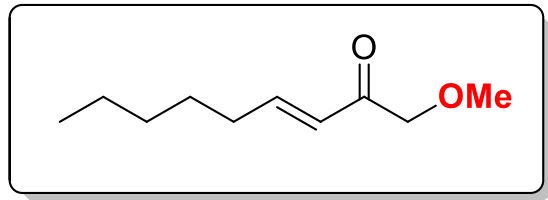

$\mathrm{m}=28 \mathrm{mg}, 82 \%$ yield, colorless oil, using pentane/EtOAc 80/20 as eluent.

${ }^{1} \mathbf{H}$ NMR (300 MHz, CDCl 3 ) $\boldsymbol{\delta}$ (ppm): 6.97 (dt, 15.9 and $\left.6.9 \mathrm{~Hz}, 1 \mathrm{H}\right), 6.24$ (dt, 15.9 and 1.3 $\mathrm{Hz}, 1 \mathrm{H}), 4.16(\mathrm{~s}, 2 \mathrm{H}), 3.42(\mathrm{~s}, 3 \mathrm{H}), 2.21(\mathrm{q}, 7.2 \mathrm{~Hz}, 2 \mathrm{H}), 1.51-1.40(\mathrm{~m}, 2 \mathrm{H}), 1.34-1.24(\mathrm{~m}, 4 \mathrm{H})$, $0.88(\mathrm{t}, 6.7 \mathrm{~Hz}, 3 \mathrm{H})$.

${ }^{13}$ C NMR (75 MHz, CDCl3) $\boldsymbol{\delta}$ (ppm): 197.0, 149.0, 125.9, 76.6, 59.3, 32.7, 31.4, 27.7, 22.4, 14.0. 
HRMS (ESI-TOF) m/z: [M+Li]+Calcd for $\mathrm{C}_{10} \mathrm{H}_{18} \mathrm{O}_{2} \mathrm{Li}$ 177.1467; Found 177.1469.

\section{Cis-2-Methoxy-4-phenylcyclohexanone 4w}

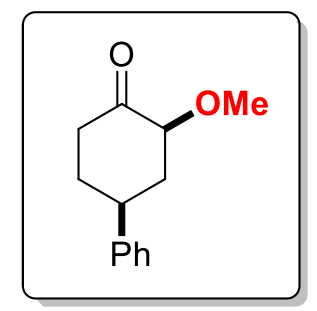

$\mathrm{m}=16.5 \mathrm{mg}, 40 \%$ yield, white solid, using pentane/EtOAc 80/20 as eluent.

Melting point: $82-84^{\circ} \mathrm{C}$

${ }^{1} \mathbf{H}$ NMR (300 MHz, CDCl 3$)$ 反 (ppm): 7.35-7.30 (m, 2H), 7.26-7.22 (m, 3H), 3.98 (dd, 12.3 and $6.1 \mathrm{~Hz}, 1 \mathrm{H}), 3.48(\mathrm{~s}, 3 \mathrm{H}), 3.15(\mathrm{tt}, 12.3$ and $3.3 \mathrm{~Hz}, 1 \mathrm{H}), 2.56-2.52(\mathrm{~m}, 3 \mathrm{H}), 2.25-2.17(\mathrm{~m}$, $1 \mathrm{H}), 2.00-1.84(\mathrm{~m}, 2 \mathrm{H})$.

${ }^{13}$ C NMR (75 MHz, CDCl 3 ) $\delta$ (ppm): 208.6, 143.5, 128.8, 126.9, 126.7, 83.5, 58.1, 42.2, 40.9, 40.3, 34.9 .

HRMS (ESI-TOF) m/z: [M-MeOH+H]+Calcd for $\mathrm{C}_{12} \mathrm{H}_{13} \mathrm{O}$ 173.0966; Found 173.0966.

\section{Cis-4-(Tert-butyl)-2-methoxycyclohexanone $4 x$}

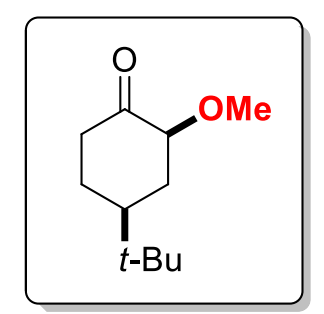

$\mathrm{m}=18.5 \mathrm{mg}, 50 \%$ yield, white solid, using pentane/EtOAc 80/20 as eluent.

Melting point: $52-54^{\circ} \mathrm{C}$

${ }^{1} \mathbf{H}$ NMR (300 MHz, CDCl 3 ) $\boldsymbol{\delta}$ (ppm): 3.80 (dd, 12.3 and $\left.6.4 \mathrm{~Hz}, 1 \mathrm{H}\right), 3.48$ (s, 3H), 2.47-2.26 (m, 2H), 2.11-2.03 (m, 1H), 1.69-1.58 (m, 2H), 1.49-1.36 (m, 2H), 0.93 (s, 9H).

${ }^{13}$ C NMR (75 MHz, CDCl 3 ) $\delta$ (ppm): 209.8, 83.8, 58.2, 46.1, 40.0, 35.6, 32.5, 28.5, 27.7.

HRMS (ESI-TOF) m/z: [M+H]+Calcd for $\mathrm{C}_{11} \mathrm{H}_{21} \mathrm{O}_{2}$ 185.1542; Found 185.1543.

\section{5-Methoxyundecan-6-one $4 y$}

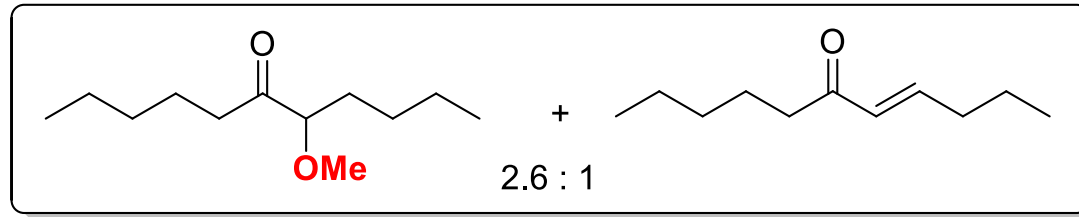

$\mathrm{m}=26 \mathrm{mg}, 47 \%$ yield $(\alpha$-alkoxyketone $)+18 \%$ yield $(\alpha, \beta$-unsaturated ketone $)$, colorless oil, using pentane/EtOAc 95/5 as eluent. 
${ }^{1} \mathbf{H}$ NMR (300 MHz, CDCl $)$ ) $(\mathbf{p p m}): 3.56(\mathrm{t}, 6.4 \mathrm{~Hz}, 1 \mathrm{H}), 3.33(\mathrm{~s}, 3 \mathrm{H}), 2.47(\mathrm{t}, 7.4 \mathrm{~Hz}, 2 \mathrm{H})$, 1.60-1.47 (m, 5H), 1.37-1.24 (m, 7H), $0.88(\mathrm{t}, 6.7 \mathrm{~Hz}, 3 \mathrm{H}), 0.88(\mathrm{t}, 6.7 \mathrm{~Hz}, 3 \mathrm{H})$.

${ }^{13}$ C NMR (75 MHz, CDCl3) $\delta$ (ppm): 213.5, 87.4, 58.2, 37.5, 31.8, 31.5, 27.4, 22.9, 22.6, $22.5,14.0,13.9$.

HRMS (ESI-TOF) m/z: [M+H]+Calcd for $\mathrm{C}_{12} \mathrm{H}_{25} \mathrm{O}_{2}$ 201.1855; Found 201.1857.

1-(6-(Tert-butyl)-1,1-dimethyl-2,3-dihydro-1H-inden-4-yl)-2-methoxyethanone 4z

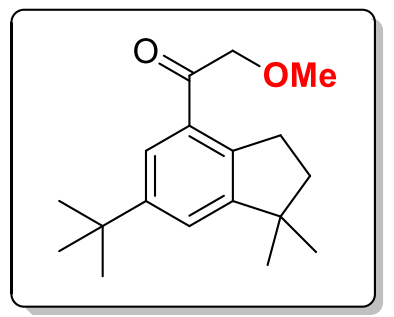

$\mathrm{m}=40 \mathrm{mg}, 73 \%$ yield, white solid, using pentane/EtOAc 93/7 as eluent.

Melting point: $88-90^{\circ} \mathrm{C}$

${ }^{1} \mathbf{H}$ NMR (300 MHz, CDCl$) \boldsymbol{\delta}$ (ppm): $7.60(\mathrm{~d}, 1.3 \mathrm{~Hz}, 1 \mathrm{H}), 7.36(\mathrm{~d}, 1.3 \mathrm{~Hz}, 1 \mathrm{H}), 4.67$ (s, 2H), 3.52 (s, 3H), 3.17 (t, 7.2 Hz, 2H), 1.94 (t, $7.2 \mathrm{~Hz}, 2 \mathrm{H}), 1.35$ (s, 9H), 1.26 (s, 6H).

${ }^{13}$ C NMR (75 MHz, CDCl3) $\delta$ (ppm): 198.0, 154.7, 150.0, 141.4, 131.3, 123.8, 123.2, 76.2, 59.5, 43.5, 41.4, 34.8, 31.5, 30.6, 28.8 .

HRMS (ESI-TOF) m/z: [M+H]+ Calcd for $\mathrm{C}_{18} \mathrm{H}_{27} \mathrm{O}_{2}$ 275.2011; Found 275.2018.

1-(1,1,2,3,3,6-Hexamethyl-2,3-dihydro-1H-inden-5-yl)-2-methoxyethanone 4aa

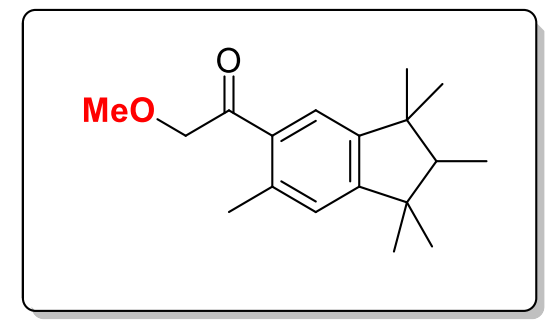

$\mathrm{m}=46 \mathrm{mg}, 84 \%$ yield, white solid, using pentane/EtOAc $90 / 10$ as eluent.

Melting point: $68-70^{\circ} \mathrm{C}$

${ }^{1} \mathbf{H}$ NMR (300 MHz, CDCl 3 ) $\delta$ (ppm): 7.33 (s, 1H), 7.03 (s, 1H), 4.58 (s, 2H), 3.50 (s, 3H), $2.51(\mathrm{~s}, 3 \mathrm{H}), 1.87$ (q, $7.2 \mathrm{~Hz}, 1 \mathrm{H}), 1.28(\mathrm{~s}, 3 \mathrm{H}), 1.27$ (s, 3H), 1.07 (s, 6H), 1.00 (d, 7.2 Hz, 3H).

${ }^{13}$ C NMR (75 MHz, CDCl3) $\delta$ (ppm): 199.9, 155.8, 149.0, 137.5, 134.1, 126.4, 122.5, 76.6, $59.4,54.4,44.9,44.5,29.1,28.7,25.9,25.6,21.4,8.5$.

HRMS (ESI-TOF) m/z: [M+H]+ Calcd for $\mathrm{C}_{18} \mathrm{H}_{27} \mathrm{O}_{2}$ 275.2011; Found 275.2006.

1-Methoxy-4-(2,6,6-trimethylcyclohex-2-en-1-yl)but-3-en-2-one 4ab 


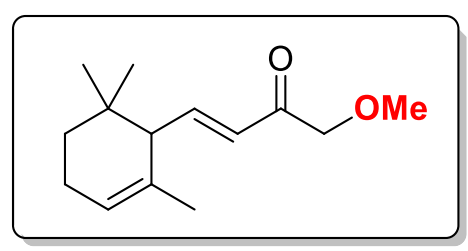

$\mathrm{m}=31 \mathrm{mg}, 70 \%$ yield, colorless oil, using pentane/EtOAc 80/20 as eluent.

${ }^{1} \mathbf{H}$ NMR (300 MHz, CDCl3) $\boldsymbol{\delta}$ (ppm): $6.79(\mathrm{dd}, 15.9$ and $9.7 \mathrm{~Hz}, 1 \mathrm{H}), 6.22(\mathrm{~d}, 15.9 \mathrm{~Hz}, 1 \mathrm{H})$, $5.48(\mathrm{~m}, 1 \mathrm{H}), 4.17(\mathrm{~s}, 2 \mathrm{H}), 3.42(\mathrm{~s}, 3 \mathrm{H}), 2.27(\mathrm{~d}, 9.7 \mathrm{~Hz}, 1 \mathrm{H}), 2.07-1.98(\mathrm{~m}, 2 \mathrm{H}), 1.54(\mathrm{~s}, 3 \mathrm{H})$, $1.50-1.40(\mathrm{~m}, 1 \mathrm{H}), 1.24-1.15(\mathrm{~m}, 1 \mathrm{H}), 0.91(\mathrm{~s}, 3 \mathrm{H}), 0.83(\mathrm{~s}, 3 \mathrm{H})$.

${ }^{13}$ C NMR (75 MHz, CDCl3) $\delta$ (ppm): 196.8, 149.4, 131.6, 127.0, 122.8, 76.7, 59.3, 54.5, 32.6, $31.1,27.9,26.8,23.0,22.8$.

HRMS (ESI-TOF) m/z: [M-MeOH+H]+ Calcd for $\mathrm{C}_{13} \mathrm{H}_{19} \mathrm{O}$ 191.1436; Found 191.1433.

(S)-4-(2-Methoxyacetyl)phenyl 2-((tert-butoxycarbonyl)amino)propanoate 4ac<smiles>COCC(=O)c1ccc(OC(=O)C(C)NC(C)(C)C)cc1</smiles>

$\mathrm{m}=41 \mathrm{mg}, 61 \%$ yield. yield, white solid, using pentane/EtOAc 85/15 as eluent.

Melting point: $102-104^{\circ} \mathrm{C}$

${ }^{1} \mathbf{H}$ NMR (300 MHz, CDCl 3$) \boldsymbol{\delta}$ (ppm): 7.98 (d, $\left.7.9 \mathrm{~Hz}, 2 \mathrm{H}\right), 7.21$ (d, $\left.7.9 \mathrm{~Hz}, 2 \mathrm{H}\right)$, 5.08-5.14 $(\mathrm{m}, 1 \mathrm{H}), 4.68(\mathrm{~s}, 2 \mathrm{H}), 4.48-4.58(\mathrm{~m}, 1 \mathrm{H}), 3.49(\mathrm{~s}, 3 \mathrm{H}), 1.56(\mathrm{~d}, 7.2 \mathrm{~Hz}, 3 \mathrm{H}), 1.45(\mathrm{~s}, 9 \mathrm{H})$.

${ }^{13}$ C NMR (75 MHz, CDCl $)$ ) (ppm): 195.1, 171.6, 155.3, 154.5, 132.6, 129.7, 121.8, 80.4, 75.3, 59.5, 49.6, 28.3, 18.2.

HRMS (ESI-TOF) m/z: [M+Na]+ Calcd for $\mathrm{C}_{17} \mathrm{H}_{23} \mathrm{O}_{6} \mathrm{Na} 360.1423$; Found 360.1434.

6,17-Dimethoxy-10,13-dimethyl-6,7,8,9,10,11,12,13,14,15,16,17-dodecahydro-1Hcyclopenta[a]phenanthren-3(2H)-one 4ad

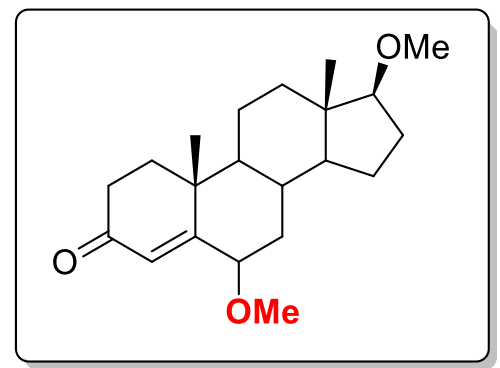

$\mathrm{m}=\mathrm{m}_{1}+\mathrm{m}_{2}=21.2+15.3=36.5 \mathrm{mg}, 55 \%$ yield, d.r. $=1.4: 1$, white solid, using pentane/EtOAc $85 / 15$ as eluent.

\section{Major diastereomer:}


${ }^{1} \mathbf{H}$ NMR (300 MHz, CDCl 3 ) $\boldsymbol{\delta}$ (ppm): $5.78(\mathrm{~s}, 1 \mathrm{H}), 3.65$ (t, $\left.2.8 \mathrm{~Hz}, 1 \mathrm{H}\right), 3.34(\mathrm{~s}, 3 \mathrm{H}), 3.23$ (t, $8.2 \mathrm{~Hz}, 1 \mathrm{H}), 3.18(\mathrm{~s}, 3 \mathrm{H}), 2.59-2.34(\mathrm{~m}, 2 \mathrm{H}), 2.07-1.88(\mathrm{~m}, 5 \mathrm{H}), 1.77-1.58(\mathrm{~m}, 3 \mathrm{H}), 1.56-1.43$ $(\mathrm{m}, 3 \mathrm{H}), 1.28(\mathrm{~s}, 3 \mathrm{H}), 1.24-1.12(\mathrm{~m}, 2 \mathrm{H}), 1.01-0.84(\mathrm{~m}, 2 \mathrm{H}), 0.81(\mathrm{~s}, 3 \mathrm{H})$.

${ }^{13}$ C NMR (75 MHz, CDCl3) $\boldsymbol{\delta}$ (ppm): 200.0, 164.3, 127.9, 90.6, 81.9, 57.9, 56.1, 54.0, 50.9, 42.9, 38.4, 37.7, 37.6, 37.1, 34.3, 30.3, 27.6, 23.2, 20.7, 17.9, 11.7.

HRMS (ESI-TOF) m/z: [M+H]+Calcd for $\mathrm{C}_{21} \mathrm{H}_{3} \mathrm{O}_{3}$ 333.2430; Found 333.2437.

Melting point: $132-134^{\circ} \mathrm{C}$

\section{Minor diastereomer:}

${ }^{1} \mathbf{H}$ NMR (300 MHz, CDCl 3$) \boldsymbol{\delta}$ (ppm): 6.10 (s, 1H), 3.77 (ddd, 12.0 and 5.4 and $\left.1.3 \mathrm{~Hz}, 1 \mathrm{H}\right)$, $3.40(\mathrm{~s}, 3 \mathrm{H}), 3.34(\mathrm{~s}, 3 \mathrm{H}), 3.23(\mathrm{t}, 8.4 \mathrm{~Hz}, 1 \mathrm{H}), 2.47-2.30(\mathrm{~m}, 2 \mathrm{H}), 2.20-2.16(\mathrm{~m}, 1 \mathrm{H}), 2.06-1.92$ $(\mathrm{m}, 3 \mathrm{H}), 1.76(\mathrm{td}, 13.3$ and $5.6 \mathrm{~Hz}, 1 \mathrm{H}), 1.68-1.44(\mathrm{~m}, 5 \mathrm{H}), 1.41-1.25(\mathrm{~m}, 2 \mathrm{H}), 1.17(\mathrm{~s}, 3 \mathrm{H})$, $1.08-0.90(\mathrm{~m}, 3 \mathrm{H}), 0.79(\mathrm{~s}, 3 \mathrm{H})$.

${ }^{13}$ C NMR (75 MHz, CDCl $)$ ) (ppm): 199.5, 169.0, 120.0, 90.4, 77.7, 57.9, 57.8, 53.9, 50.5, $42.9,39.1,37.9,37.5,36.1,34.1,33.8,27.6,23.3,20.9,18.5,11.6$.

HRMS (ESI-TOF) m/z: [M+H]+ Calcd for $\mathrm{C}_{21} \mathrm{H}_{3} \mathrm{O}_{3}$ 333.2430; Found 333.2437.

8-Methoxy-4,4a-dimethyl-6-(prop-1-en-2-yl)-4,4a,5,6,7,8-hexahydronaphthalen-2(3H)one 4ae

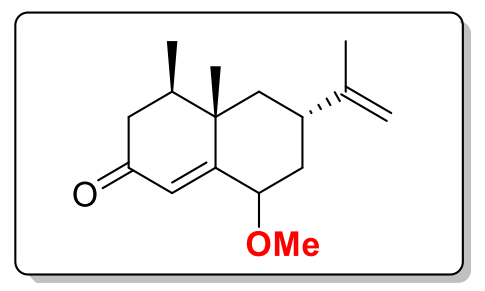

$\mathrm{m}=\mathrm{m}_{1}+\mathrm{m}_{2}=28+13=41 \mathrm{mg}, 83 \%$ yield, d.r. $=2.9: 1$, white solid, using pentane $/$ EtOAc 80/20 as eluent.

Major diastereomer:

${ }^{1} \mathbf{H}$ NMR (300 MHz, CDCl $)$ ) (ppm): 5.82 (s, 1H), 4.75-4.72 (m, 2H), $3.76(\mathrm{t}, 2.6 \mathrm{~Hz}, 1 \mathrm{H})$, $3.19(\mathrm{~s}, 3 \mathrm{H}), 2.68(\mathrm{tt}, 13.1$ and $2.6 \mathrm{~Hz}, 1 \mathrm{H}), 2.42-2.23(\mathrm{~m}, 2 \mathrm{H}), 2.16-2.07(\mathrm{~m}, 1 \mathrm{H}), 2.04-1.92$ $(\mathrm{m}, 2 \mathrm{H}), 1.74(\mathrm{~s}, 3 \mathrm{H}), 1.50(\mathrm{td}, 13.1 \mathrm{and} 3.1 \mathrm{~Hz}, 1 \mathrm{H}), 1.21(\mathrm{~s}, 3 \mathrm{H}), 1.12-1.06(\mathrm{~m}, 1 \mathrm{H}), 0.94(\mathrm{~d}$, $6.9 \mathrm{~Hz}, 3 \mathrm{H})$.

${ }^{13}$ C NMR (75 MHz, CDCl3) $\boldsymbol{\delta}$ (ppm): 200.0, 163.8, 149.0, 128.7, 109.3, 82.3, 56.1, 43.9, 42.4, 41.3, 39.1, 37.3, 34.6, 21.0, 16.7, 14.6.

HRMS (ESI-TOF) m/z: [M+H]+ Calcd for $\mathrm{C}_{16} \mathrm{H}_{25} \mathrm{O}_{2}$ 249.1855; Found 249.1862.

Melting point: $43-45^{\circ} \mathrm{C}$ 


\section{Minor diastereomer:}

${ }^{1} \mathbf{H}$ NMR (300 MHz, CDCl 3 ) $\boldsymbol{\delta}$ (ppm): 6.19 (s, 1H), 4.76-4.74 (m, 2H), 3.90 (ddd, 12.3 and 5.1 and $1.8 \mathrm{~Hz}, 1 \mathrm{H}), 3.43(\mathrm{~s}, 3 \mathrm{H}), 2.41-2.23(\mathrm{~m}, 4 \mathrm{H}), 2.08-2.01(\mathrm{~m}, 1 \mathrm{H}), 1.99-1.91(\mathrm{~m}, 1 \mathrm{H})$, $1.73(\mathrm{~s}, 3 \mathrm{H}), 1.34-1.30(\mathrm{~m}, 1 \mathrm{H}), 1.14-1.09(\mathrm{~m}, 1 \mathrm{H}), 1.12(\mathrm{~s}, 3 \mathrm{H}), 0.97(\mathrm{~d}, 6.7 \mathrm{~Hz}, 3 \mathrm{H})$.

${ }^{13} \mathbf{C}$ NMR (75 MHz, CDCl3) $\delta$ (ppm): 199.6, 168.5, 148.0, 121.2, 109.9, 78.2, 57.9, 44.1, 41.9, 40.9, 39.7, 38.5, 37.4, 20.7, 17.6, 15.2.

HRMS (ESI-TOF) m/z: [M+H]+ Calcd for $\mathrm{C}_{16} \mathrm{H}_{25} \mathrm{O}_{2}$ 249.1855; Found 249.1860.

\section{2-Ethoxy-1-(4-fluorophenyl)ethanone 4af}

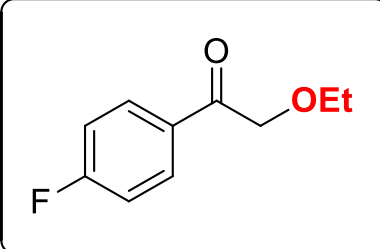

$\mathrm{m}=24 \mathrm{mg}$, 66\% yield, colorless oil, using pentane/EtOAc 85/15 as eluent.

${ }^{1} \mathbf{H}$ NMR (300 MHz, CDCl $\left.\mathbf{3}\right) \boldsymbol{\delta}$ (ppm): 7.99 (dd, 8.7 and $\left.5.4 \mathrm{~Hz}, 2 \mathrm{H}\right), 7.14(\mathrm{t}, 8.4 \mathrm{~Hz}, 2 \mathrm{H}), 4.69$ (s, 2H), $3.63(\mathrm{q}, 6.9 \mathrm{~Hz}, 2 \mathrm{H}), 1.28(\mathrm{t}, 6.9 \mathrm{~Hz}, 3 \mathrm{H})$.

${ }^{13}$ C NMR (75 MHz, CDCl 3 ) $\delta$ (ppm): 195.2, 165.9 (d, 255.2 Hz), 131.4 (d, 2.7 Hz), 130.8 (d, 9.3 Hz), 115.9 (d, 22.0 Hz), 73.7, 67.3, 15.1.

${ }^{19}$ F NMR (282 MHz, CDCl 3$) \delta$ (ppm): -104.74.

HRMS (ESI-TOF) m/z: [M+H]+ Calcd for $\mathrm{C}_{10} \mathrm{H}_{12} \mathrm{O}_{2} \mathrm{~F}$ 183.0821; Found 183.0821.

\section{3-(2-(4-Fluorophenyl)-2-oxoethoxy)propanenitrile 4ag}

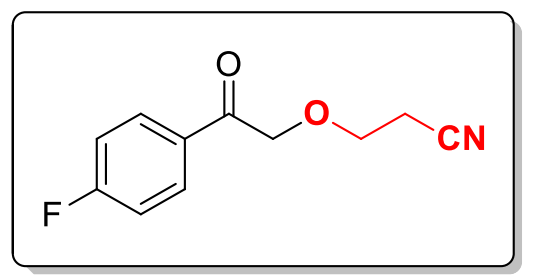

$\mathrm{m}=25 \mathrm{mg}, 60 \%$ yield, white solid, using pentane/EtOAc 75/25 as eluent.

${ }^{1} \mathbf{H}$ NMR (300 MHz, CDCl $)$ ) $\boldsymbol{\delta}$ (ppm): $7.96(\mathrm{dd}, 9.0$ and $5.6 \mathrm{~Hz}, 2 \mathrm{H}), 7.16(\mathrm{t}, 8.4 \mathrm{~Hz}, 2 \mathrm{H}), 4.80$ (s, 2H), $3.83(\mathrm{t}, 6.4 \mathrm{~Hz}, 2 \mathrm{H}), 2.71(\mathrm{t}, 6.4 \mathrm{~Hz}, 2 \mathrm{H})$.

${ }^{13}$ C NMR (75 MHz, CDCl3) $\delta$ (ppm): 194.1, 166.1 (d, 256.3 Hz), 130.9 (d, 2.7 Hz), 130.7 (d, 9.3 Hz), 117.7, 116.6 (d, 22.0 Hz), 73.9, 66.3, 19.1.

${ }^{19}$ F NMR (282 MHz, CDCl3) $\delta$ (ppm): -103.86.

HRMS (ESI-TOF) m/z: [M+H]+ Calcd for $\mathrm{C}_{11} \mathrm{H}_{11} \mathrm{O}_{2} \mathrm{NF}$ 208.0774; Found 208.0770. 


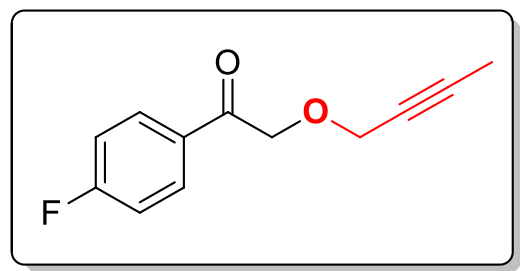

$\mathrm{m}=16.5 \mathrm{mg}, 40 \%$ yield, colorless oil, using pentane/EtOAc 80/20 as eluent.

${ }^{1} \mathbf{H}$ NMR (300 MHz, CDCl $)$ ) $(\mathbf{p p m}): 8.00$ (dd, 9.0 and $\left.5.6 \mathrm{~Hz}, 2 \mathrm{H}\right), 7.15(\mathrm{t}, 8.4 \mathrm{~Hz}, 2 \mathrm{H}), 4.79$ (s, 2H), $4.30(\mathrm{q}, 2.3 \mathrm{~Hz}, 2 \mathrm{H}), 1.85(\mathrm{t}, 2.3 \mathrm{~Hz}, 3 \mathrm{H})$.

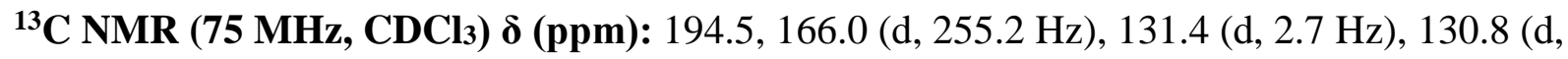
9.3 Hz), 115.9 (d, 22.0 Hz), 84.0, 74.2, 71.7, 59.1, 3.7.

${ }^{19}$ F NMR (282 MHz, CDCl3) $\delta$ (ppm): -104.57.

HRMS (ESI-TOF) m/z: [M+Na]+Calcd for $\mathrm{C}_{12} \mathrm{H}_{11} \mathrm{O}_{2} \mathrm{FNa} 229.0641$; Found 229.0638.

1-(4-Fluorophenyl)-2-(2-phenoxyethoxy)ethanone 4ai

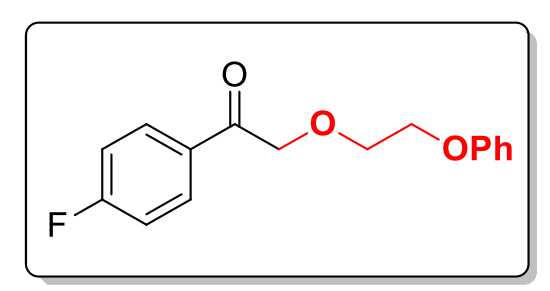

$\mathrm{m}=35 \mathrm{mg}, 64 \%$ yield, white solid, using pentane/EtOAc 80/20 as eluent.

Melting point: $77-79^{\circ} \mathrm{C}$

${ }^{1} \mathbf{H}$ NMR (300 MHz, CDCl 3 ) $\boldsymbol{\delta}$ (ppm): 7.99 (dd, 8.7 and $\left.5.4 \mathrm{~Hz}, 2 \mathrm{H}\right), 7.28$ (t, $\left.7.4 \mathrm{~Hz}, 2 \mathrm{H}\right), 7.13$ $(\mathrm{t}, 8.4 \mathrm{~Hz}, 2 \mathrm{H}), 6.95(\mathrm{t}, 7.4 \mathrm{~Hz}, 1 \mathrm{H}), 6.93-6.87(\mathrm{~m}, 2 \mathrm{H}), 4.85$ (s, 2H), 4.19 (t, $4.6 \mathrm{~Hz}, 2 \mathrm{H}), 3.97$ (t, $4.6 \mathrm{~Hz}, 2 \mathrm{H})$.

${ }^{13}$ C NMR (75 MHz, CDCl3) $\boldsymbol{\delta}$ (ppm): 195.0, 166.0 (d, 255.2 Hz), 158.6, 131.3 (d, $\left.2.7 \mathrm{~Hz}\right)$, 130.8 (d, 9.3 Hz), 129.5, 121.1, 115.9 (d, 22.0 Hz), 114.6, 74.4, 70.2, 67.4.

HRMS (ESI-TOF) m/z: [M+H]+ Calcd for $\mathrm{C}_{16} \mathrm{H}_{16} \mathrm{O}_{3} \mathrm{~F} 275.1083$; Found 275.1083.

\section{Methyl 3-(2-(3-phenylpropoxy)acetyl)benzoate 4aj}

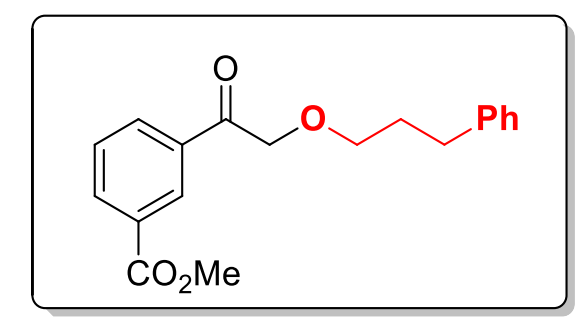

$\mathrm{m}=45 \mathrm{mg}$, 72\% yield, white solid, using pentane/EtOAc 80/20 as eluent.

Melting point: $88-90^{\circ} \mathrm{C}$ 
${ }^{1} \mathbf{H}$ NMR (300 MHz, CDCl 3 ) $\boldsymbol{\delta}$ (ppm): 8.61 (s, 1H), 8.29 (d, $\left.7.7 \mathrm{~Hz}, 1 \mathrm{H}\right), 8.20(\mathrm{~d}, 7.7 \mathrm{~Hz}, 1 \mathrm{H})$, $7.61(\mathrm{t}, 7.7 \mathrm{~Hz}, 1 \mathrm{H}), 7.30-7.21(\mathrm{~m}, 5 \mathrm{H}), 4.79(\mathrm{~s}, 2 \mathrm{H}), 3.99(\mathrm{~s}, 3 \mathrm{H}), 3.64(\mathrm{t}, 6.4 \mathrm{~Hz}, 2 \mathrm{H}), 2.77(\mathrm{t}$, $6.4 \mathrm{~Hz}, 2 \mathrm{H}), 2.08-2.00(\mathrm{~m}, 2 \mathrm{H})$.

${ }^{13}$ C NMR (75 MHz, CDCl3) $\boldsymbol{\delta}$ (ppm): 196.0, 166.2, 141.7, 135.2, 134.3, 132.3, 130.8, 129.2, 129.0, 128.5, 128.4, 125.9, 74.1, 71.2, 52.5, 32.2, 31.2.

HRMS (ESI-TOF) m/z: [M+H]+ Calcd for $\mathrm{C}_{19} \mathrm{H}_{21} \mathrm{O}_{4}$ 313.1440; Found 313.1429.

Methyl 3-(2-(3-(benzoyloxy)propoxy)acetyl)benzoate 4ak

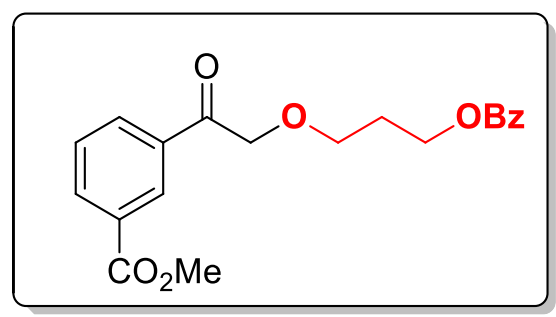

$\mathrm{m}=29 \mathrm{mg}, 41 \%$ yield, white solid, using pentane/EtOAc 75/25 as eluent.

Melting point: $95-97^{\circ} \mathrm{C}$

${ }^{1} \mathbf{H}$ NMR (300 MHz, CDCl $)$ 反 (ppm): $8.56(\mathrm{~s}, 1 \mathrm{H}), 8.22(\mathrm{~d}, 7.7 \mathrm{~Hz}, 1 \mathrm{H}), 8.15(\mathrm{~d}, 7.7 \mathrm{~Hz}, 1 \mathrm{H})$, $8.00(\mathrm{~d}, 7.4 \mathrm{~Hz}, 2 \mathrm{H}), 7.59-7.51(\mathrm{~m}, 2 \mathrm{H}), 7.42(\mathrm{t}, 7.9 \mathrm{~Hz}, 2 \mathrm{H}), 4.78(\mathrm{~s}, 2 \mathrm{H}), 4.45(\mathrm{t}, 6.1 \mathrm{~Hz}, 2 \mathrm{H})$, $3.95(\mathrm{~s}, 3 \mathrm{H}), 3.75(\mathrm{t}, 6.1 \mathrm{~Hz}, 2 \mathrm{H}), 2.13$ (quint, $6.1 \mathrm{~Hz}, 2 \mathrm{H})$.

${ }^{13}$ C NMR (75 MHz, CDCl3) $\boldsymbol{\delta}$ (ppm): 195.9, 166.5, 166.1, 135.1, 134.4, 132.9, 132.2, 130.8, 130.3, 129.6, 129.1, 129.0, 128.4, 74.2, 68.3, 61.9, 52.5, 29.2 .

HRMS (ESI-TOF) m/z: [M+H]+Calcd for $\mathrm{C}_{20} \mathrm{H}_{21} \mathrm{O}_{6}$ 357.1338; Found 357.1343.

Methyl 3-(2-(3-(phenylsulfonyl)propoxy)acetyl)benzoate 4al

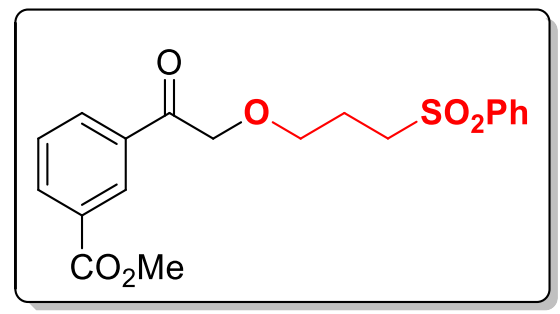

$\mathrm{m}=40 \mathrm{mg}$, 53\% yield, white solid, using pentane/EtOAc 50/50 as eluent.

${ }^{1}$ H NMR (300 MHz, CDCl $)$ ) (ppm): 8.49 (s, 1H), 8.24 (d, 7.7 Hz, 1H), 8.09 (d, 7.7 Hz, 1H), $7.91(\mathrm{~d}, 7.4 \mathrm{~Hz}, 2 \mathrm{H}), 7.67-7.53(\mathrm{~m}, 4 \mathrm{H}), 4.73(\mathrm{~s}, 2 \mathrm{H}), 3.95$ (s, 3H), 3.66 (t, $5.9 \mathrm{~Hz}, 2 \mathrm{H}), 3.32-$ $3.26(\mathrm{~m}, 2 \mathrm{H}), 2.13-2.04(\mathrm{~m}, 2 \mathrm{H})$.

${ }^{13}$ C NMR (75 MHz, CDCl 3 ) $\boldsymbol{\delta}$ (ppm): 195.4, 166.1, 139.1, 134.9, 134.5, 133.8, 132.0, 130.8, 129.3, 129.1, 128.9, 128.1, 73.7, 69.3, 53.3, 52.6, 23.3.

HRMS (ESI-TOF) m/z: [M+H]+Calcd for $\mathrm{C}_{19} \mathrm{H}_{21} \mathrm{O}_{6} \mathrm{~S} 377.1059$; Found 377.1057. 


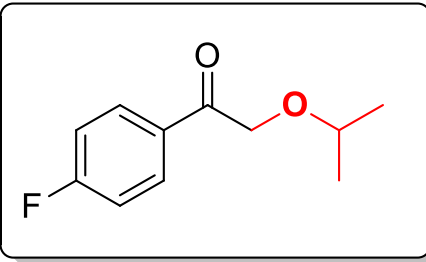

$\mathrm{m}=24 \mathrm{mg}$, 61\% yield, colorless oil, using pentane/EtOAc 85/15 as eluent.

${ }^{1} \mathbf{H}$ NMR (300 MHz, CDCl $) \boldsymbol{\delta}$ (ppm): $8.00(\mathrm{dd}, 8.7$ and $5.6 \mathrm{~Hz}, 2 \mathrm{H}), 7.13(\mathrm{t}, 8.4 \mathrm{~Hz}, 2 \mathrm{H}), 4.67$ (s, 2H), 3.71 (quint, $6.1 \mathrm{~Hz}, 1 \mathrm{H}), 1.23(\mathrm{~d}, 6.1 \mathrm{~Hz}, 6 \mathrm{H})$.

${ }^{13}$ C NMR (75 MHz, CDCl 3 ) $\delta$ (ppm): 195.6, 165.9 (d, 254.7 Hz), 131.6 (d, 2.7 Hz), 130.9 (d, 9.3 Hz), 115.8 (d, 22.0 Hz), 72.8, 71.5, 21.9.

${ }^{19}$ F NMR (282 MHz, CDCl3) $\delta$ (ppm): -104.95.

HRMS (ESI-TOF) m/z: [M+Na]+ Calcd for $\mathrm{C}_{11} \mathrm{H}_{13} \mathrm{O}_{2} \mathrm{FNa} 219.0797$; Found 219.0794.

\section{2-((1,3-Difluoropropan-2-yl)oxy)-1-(4-fluorophenyl)ethanone 4an}

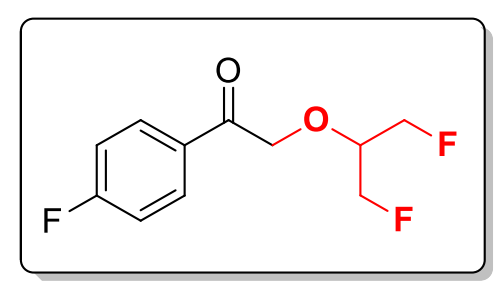

$\mathrm{m}=37.5 \mathrm{mg}, 81 \%$ yield, colorless oil, using pentane/EtOAc 80/20 as eluent.

${ }^{1} \mathbf{H}$ NMR (300 MHz, CDCl $)$ ) $\mathbf{~ ( p p m ) : ~} 7.95$ (dd, 8.7 and 5.4 Hz, 2H), 7.15 (t, 8.4 Hz, 2H), 4.96 (s, 2H), $4.72(\mathrm{~d}, 4.9 \mathrm{~Hz}, 2 \mathrm{H}), 4.56(\mathrm{~d}, 4.9 \mathrm{~Hz}, 2 \mathrm{H}), 4.04-3.88(\mathrm{~m}, 1 \mathrm{H})$.

${ }^{13}$ C NMR (75 MHz, CDCl 3 ) $\delta$ (ppm): 194.3, 166.1 (d, 255.8 Hz), 131.0 (d, 2.7 Hz), 130.6 (d, 9.3 Hz), 116.0 (d, 22.0 Hz), 82.3 (d, $170.7 \mathrm{~Hz}), 82.2$ (d, $170.7 \mathrm{~Hz}), 77.4$ (t, 19.8 Hz), 73.4.

${ }^{19}$ F NMR (282 MHz, CDCl3) $\boldsymbol{\delta}$ (ppm): -104.16 (1F), -231.64 (2F).

HRMS (ESI-TOF) m/z: [M+Na]+ Calcd for $\mathrm{C}_{11} \mathrm{H}_{12} \mathrm{O}_{2} \mathrm{~F}_{3}$ 233.0789; Found 233.0798.

\section{3-(2-Methoxyhexanoyl)oxazolidin-2-one 7a}

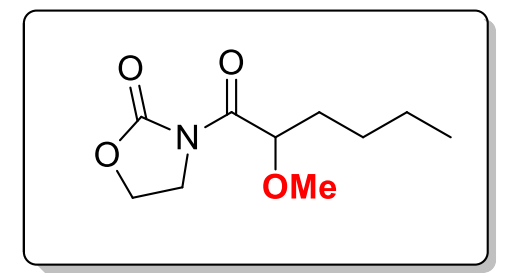

$\mathrm{m}=32 \mathrm{mg}, 74 \%$ yield, white solid, using pentane/EtOAc 70/30 as eluent.

Melting point: $51-53^{\circ} \mathrm{C}$

${ }^{1} \mathbf{H}$ NMR (300 MHz, CDCl $)$ ) $(\mathbf{p p m}): 4.87(\mathrm{dd}, 7.9$ and $3.6 \mathrm{~Hz}, 1 \mathrm{H}), 4.46(\mathrm{t}, 8.2 \mathrm{~Hz}, 2 \mathrm{H})$, 4.14-3.99 (m, 2H), 3.35 (s, 3H), 1.77-1.58 (m, 2H), 1.49-1.39 (m, 2H), 1.38-1.23 (m, 2H), 0.88 (t, 7.4 Hz, 3H). 
${ }^{13}$ C NMR (75 MHz, CDCl3) $\boldsymbol{\delta}$ (ppm): 173.3, 153.2, 79.7, 62.7, 58.1, 42.5, 32.7, 27.6, 22.4, 14.0.

HRMS (ESI-TOF) m/z: [M+H]+Calcd for $\mathrm{C}_{10} \mathrm{H}_{18} \mathrm{O}_{4} \mathrm{~N}$ 216.1236; Found 216.1237.

3-(6-Bromo-2-methoxyhexanoyl)oxazolidin-2-one 7b

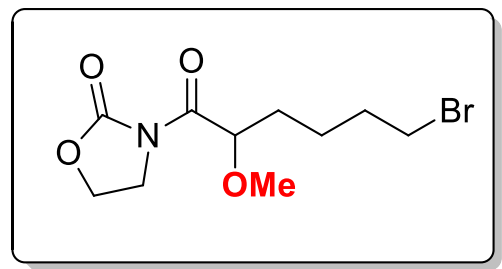

$\mathrm{m}=40 \mathrm{mg}, 68 \%$ yield, white solid, using pentane/EtOAc 70/30 as eluent.

Melting point: $68-70^{\circ} \mathrm{C}$

${ }^{1} \mathbf{H}$ NMR (300 MHz, CDCl 3$) \boldsymbol{\delta}$ (ppm): 4.87-4.84 (m, 1H), $4.48(\mathrm{t}, 7.9 \mathrm{~Hz}, 2 \mathrm{H})$, 4.16-3.97 (m, $2 \mathrm{H}), 3.41(\mathrm{t}, 6.7 \mathrm{~Hz}, 2 \mathrm{H}), 3.36(\mathrm{~s}, 3 \mathrm{H}), 1.95-1.86(\mathrm{~m}, 2 \mathrm{H}), 1.78-1.63(\mathrm{~m}, 4 \mathrm{H})$.

${ }^{13}$ C NMR (75 MHz, CDCl3) $\boldsymbol{\delta}$ (ppm): 172.9, 153.3, 79.4, 62.7, 58.2, 42.5, 33.5, 32.2, 31.9, 24.1.

HRMS (ESI-TOF) m/z: [M+H]+ Calcd for $\mathrm{C}_{10} \mathrm{H}_{17} \mathrm{O}_{4} \mathrm{NBr} 294.0341$; Found 294.0345.

3-(2-Methoxy-3-(methylthio)propanoyl)oxazolidin-2-one 7c<smiles>COCC(OC)C(=O)N1CCOC1=O</smiles>

$\mathrm{m}=26 \mathrm{mg}$, 59\% yield, colorless oil, using pentane/EtOAc 50/50 as eluent.

${ }^{1}$ H NMR (300 MHz, CDCl3) $\boldsymbol{\delta}$ (ppm): 5.18-5.14 (m, 1H), $4.48(\mathrm{t}, 8.1 \mathrm{~Hz}, 2 \mathrm{H}), 4.16-4.00$ (m, 2H), 3.43 (s, 3H), 2.93 (dd, 14.0 and $5.0 \mathrm{~Hz}, 1 \mathrm{H}), 2.81$ (dd, 14.0 and $6.8 \mathrm{~Hz}, 1 \mathrm{H}), 2.21(\mathrm{~s}, 3 \mathrm{H})$.

${ }^{13}$ C NMR (75 MHz, $\mathbf{C D C l}_{3}$ ) $\delta$ (ppm): 171.3, 153.3, 79.0, 62.7, 58.4, 42.6, 35.9, 16.6.

HRMS (ESI-TOF) m/z: [M+H]+Calcd for $\mathrm{C}_{8} \mathrm{H}_{14} \mathrm{O}_{4} \mathrm{NS} 220.0644$; Found 220.0639.

\section{3-(4,4,4-trifluoro-2-methoxybutanoyl)oxazolidin-2-one 7d}

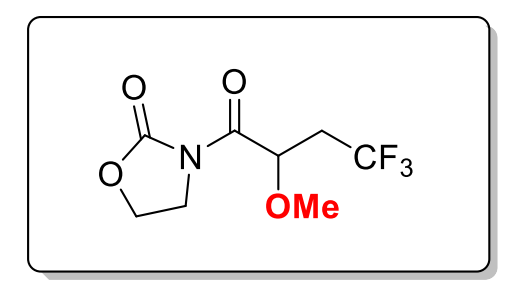

$\mathrm{m}=25 \mathrm{mg}$, 52\% yield, colorless oil, using pentane/EtOAc 70/30 as eluent.

${ }^{1} \mathbf{H}$ NMR (300 MHz, CDCl3) $\boldsymbol{\delta}$ (ppm): 5.23 (t, $\left.5.7 \mathrm{~Hz}, 1 \mathrm{H}\right), 4.51$ (t, 8.1 Hz, 2H), 4.18-4.03 (m, $2 \mathrm{H}), 3.44$ (s, 3H), 2.77-2.50 (m, 2H). 
${ }^{13}$ C NMR (75 MHz, CDCl3) $\boldsymbol{\delta}$ (ppm): 170.1, 153.1, 126.2 (q, 276.6 Hz), 73.6, 62.8, 58.4, 42.6, $36.2(\mathrm{q}, 29.1 \mathrm{~Hz})$.

${ }^{19}$ F NMR (282 MHz, CDCl3) $\delta$ (ppm): -63.2 (t, $10.3 \mathrm{~Hz}$,)

HRMS (ESI-TOF) m/z: [M+H]+ Calcd for $\mathrm{C}_{8} \mathrm{H}_{11} \mathrm{O}_{4} \mathrm{NF}_{3} 242.0640$; Found 242.0633.

\section{3-(2-Methoxyundec-10-enoyl)oxazolidin-2-one 7e}

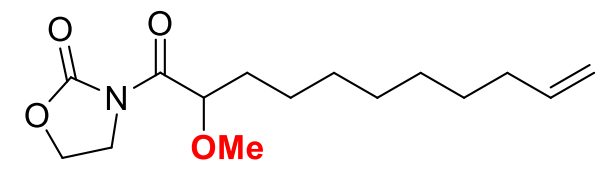

$\mathrm{m}=35 \mathrm{mg}, 62 \%$ yield, white solid, using pentane/EtOAc 70/30 as eluent.

Melting point: $48-50^{\circ} \mathrm{C}$

${ }^{1}$ H NMR (300 MHz, CDCl$)$ ) $(\mathbf{p p m})$ : 5.84-5.75 (m, 1H), 5.00-4.85 (m, 3H), 4.46 (t, $8.1 \mathrm{~Hz}$, $2 \mathrm{H}), 4.15-3.96(\mathrm{~m}, 2 \mathrm{H}), 3.35(\mathrm{~s}, 3 \mathrm{H}), 2.03-1.98(\mathrm{~m}, 2 \mathrm{H}), 1.76-1.56(\mathrm{~m}, 2 \mathrm{H}), 1.48-1.22(\mathrm{~m}, 10 \mathrm{H})$.

${ }^{13}$ C NMR (75 MHz, CDCl 3 ) $\delta$ (ppm): 173.3, 153.2, 139.2, 114.1, 79.7, 62.7, 58.1, 42.5, 33.8, 33.0, 29.3, 29.2, 29.0, 28.9, 25.4 .

HRMS (ESI-TOF) m/z: [M+H]+Calcd for $\mathrm{C}_{15} \mathrm{H}_{26} \mathrm{O}_{4} \mathrm{~N} 284.1862$; Found 284.1851.

\section{3-(2-Cyclohexyl-2-methoxyacetyl)oxazolidin-2-one $7 f$}<smiles>COC(C(=O)N1CCOC1=O)C1CCCCC1</smiles>

$\mathrm{m}=21 \mathrm{mg}$, 44\% yield, white solid, using pentane/EtOAc 70/30 as eluent.

Melting point: $48-50^{\circ} \mathrm{C}$

${ }^{1} \mathbf{H}$ NMR (300 MHz, CDCl 3$) \boldsymbol{\delta}$ (ppm): 4.83 (d, $\left.3.8 \mathrm{~Hz}, 1 \mathrm{H}\right), 4.48$ (t, 8.2 Hz, 2H), 4.17-3.98 (m, 2H), 3.35 (s, 3H), 1.79-1.56 (m, 6H), 1.34-1.12 (m, 5H).

${ }^{13}$ C NMR (75 MHz, CDCl3) $\boldsymbol{\delta}$ (ppm): 173.0, 153.3, 83.5, 62.5, 58.6, 42.6, 41.2, 29.4, 26.8, 26.3, 26.1, 26.1.

HRMS (ESI-TOF) m/z: [M+H]+ Calcd for $\mathrm{C}_{12} \mathrm{H}_{20} \mathrm{O}_{4} \mathrm{~N}$ 242.1392; Found 242.1388. 


\section{5-((3,5-dimethylphenoxy)methyl)-3-(2-methoxyhexanoyl)oxazolidin-2-one $7 \mathrm{~g}$}

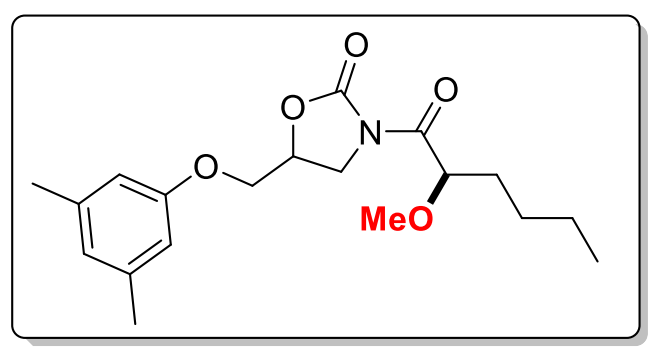

$\mathrm{m}=39 \mathrm{mg}, 56 \%$ yield, white solid, using pentane/EtOAc $60 / 40$ as eluent.

Melting point: $122-124^{\circ} \mathrm{C}$

${ }^{1}$ H NMR (300 MHz, CDCl3) $\boldsymbol{\delta}$ (ppm): 6.66 (s, 1H), 6.52 (s, 2H), 4.96-4.88 (m, 2H), 4.24-4.10 $(\mathrm{m}, 4 \mathrm{H}), 3.40(\mathrm{~s}, 3 \mathrm{H}), 2.29(\mathrm{~s}, 6 \mathrm{H}), 1.84-1.61(\mathrm{~m}, 2 \mathrm{H}), 1.53-1.43(\mathrm{~m}, 2 \mathrm{H}), 1.38-1.26(\mathrm{~m}, 2 \mathrm{H})$, $0.91(\mathrm{t}, 7.2 \mathrm{~Hz}, 3 \mathrm{H})$.

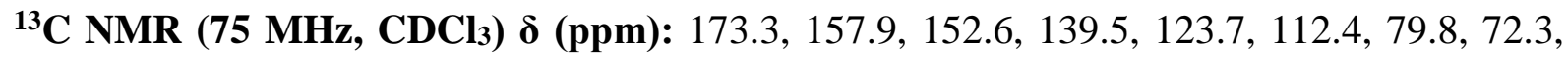
67.7, 58.1, 44.6, 32.8, 27.6, 22.5, 21.4, 14.0.

HRMS (ESI-TOF) m/z: [M+H]+ Calcd for $\mathrm{C}_{19} \mathrm{H}_{28} \mathrm{O}_{5} \mathrm{~N}$ 350.1967; Found 350.1974.

\section{Tetrahydrofuran-2-carbaldehyde 9}

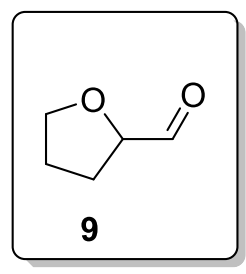

Procedure : In a dry tube, 8 (48 mg, $0.09 \mathrm{mmol}, 1$ equiv.), $\operatorname{Ir}(\text { ppy })_{3}(1.3 \mathrm{mg}, 0.002 \mathrm{mmol}, 2$ mol\%) and $\mathrm{K}_{2} \mathrm{HPO}_{4}\left(31 \mathrm{mg}, 0.18 \mathrm{mmol}, 2\right.$ equiv.) were introduced. Then $\mathrm{CD}_{3} \mathrm{CN}(0.9 \mathrm{~mL})$ was added and the reaction mixture was degazed under argon and agitated under blue LEDs irradiation $(450 \mathrm{~nm})$ at room temperature during $18 \mathrm{~h}$. Due to the volatility of compound 9, attempts for the purification of the product were unsuccessful, so the yield was evaluated using ${ }^{1} \mathrm{H}$ NMR (300 MHz) of the crude reaction mixture, using 1,3,5-trimethoxybenzene as internal standard in $\mathrm{CD}_{3} \mathrm{CN}$. A yield of $52 \%$ was found for tetrahydrofuran-2-carbaldehyde 9.

Spectroscopic data are in accordance with literature. ${ }^{[5]}$ 


\section{Crude ${ }^{1} \mathrm{H}$ NMR (300 MHz, $\left.\mathrm{CDCl}_{3}\right)$}

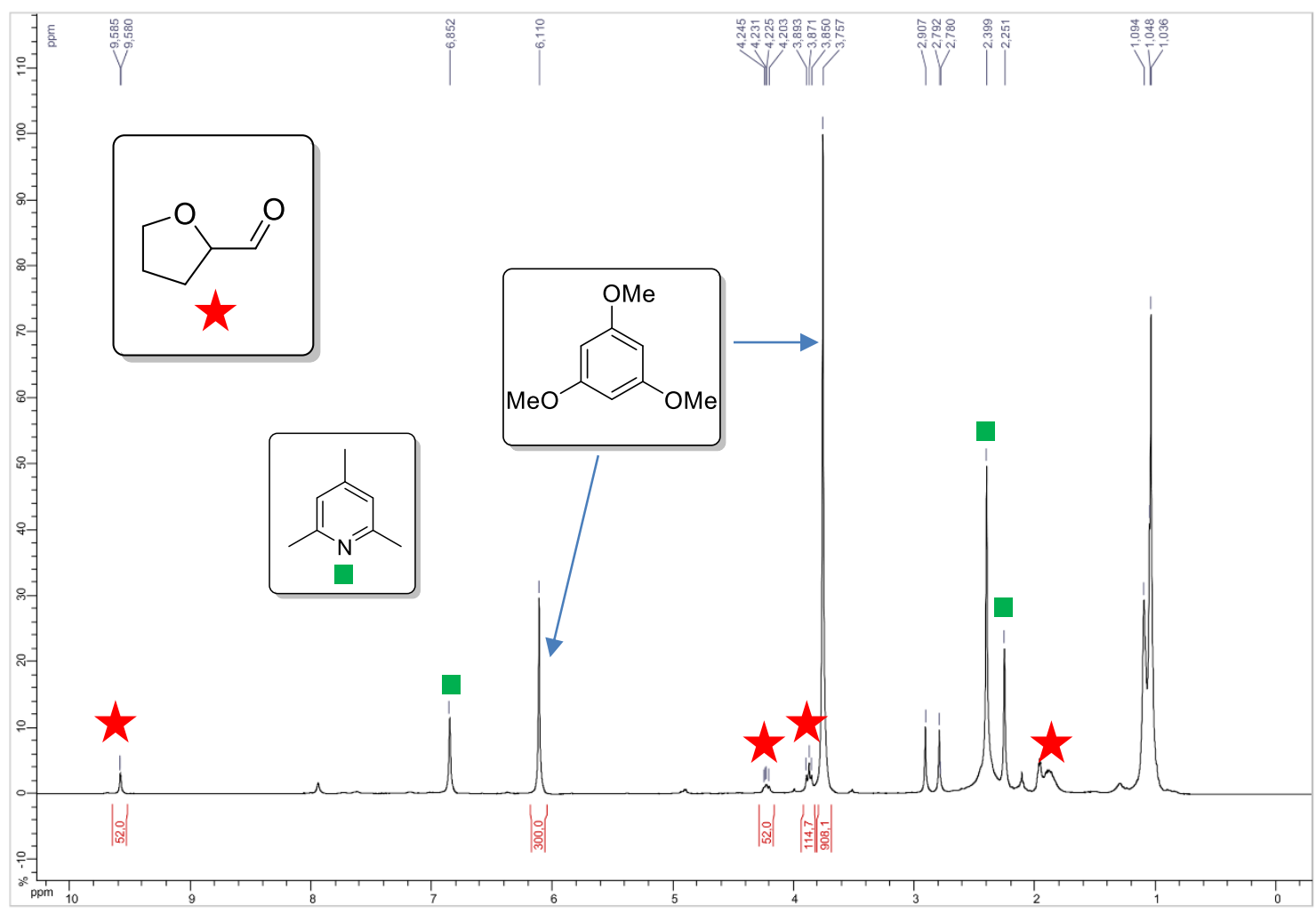

\section{Competition with 1,5-HAT:}
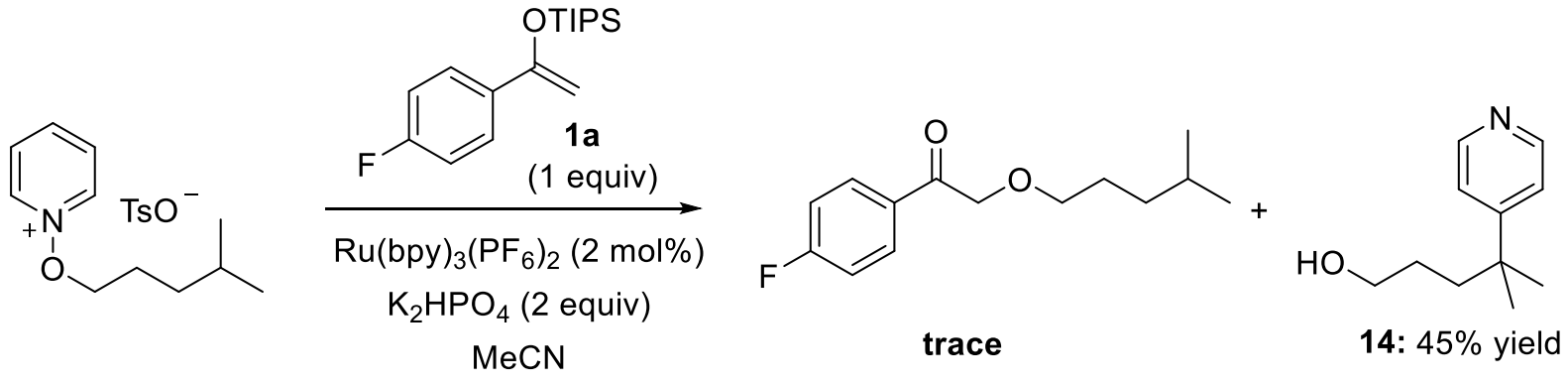

\section{4-Methyl-4-(pyridin-4-yl)pentan-1-ol 14}

Prepared according to general procedure GP3. $16 \mathrm{mg}$ (Yield $=45 \%$ ), Colorless oil. Spectroscopic data are in accordance with literature. ${ }^{[6]}$

${ }^{1}$ H NMR (200 MHz, acetone-d6) $\delta 8.48(\mathrm{~d}, J=5.8 \mathrm{~Hz}, 2 \mathrm{H}), 7.34(\mathrm{~d}, J=5.8 \mathrm{~Hz}, 2 \mathrm{H}), 3.57(\mathrm{~s}$, $1 \mathrm{H}), 3.46(\mathrm{t}, J=6.3 \mathrm{~Hz}, 2 \mathrm{H}), 1.79-1.60(\mathrm{~m}, 2 \mathrm{H}), 1.31(\mathrm{~s}, 6 \mathrm{H}), 1.31-1.20(\mathrm{~m}, 2 \mathrm{H})$. 


\section{Luminescence quenching experiments}

Rates of quenching $\left(\mathrm{k}_{\mathrm{q}}\right)$ were determined using Stern-Volmer kinetics:

$$
\mathrm{I}_{0} / \mathrm{I}=\mathrm{k}_{\mathrm{q}} \tau_{0}[\text { quencher }]+1
$$

Where $\mathrm{I}_{0}$ is the luminescene intensity without the quencher, $\mathrm{I}$ is the intensity with the quencher, and $\tau_{0}$ is the excited state lifetime of the photocatalyst.

The following stock solutions were prepared in distilled MeCN and degassed by three freezepump-thaw cycles.

General procedure: A stock solution of $\mathbf{3 a}$ was prepared by dissolving $\mathrm{Ru}(\mathrm{bpy})_{3}\left(\mathrm{PF}_{6}\right)_{2} \mathbf{3 d}(25$ $\mu \mathrm{mol})$ in $10 \mathrm{~mL}$ of $\mathrm{MeCN}$. Of this solution, $0.1 \mathrm{~mL}$ were further diluted with $\mathrm{MeCN}$ to give a total volume of $10 \mathrm{~mL}$. Concentration of $[\mathbf{3 d}]=2.5 \times 10^{-5} \mathrm{M}$. A stock solution of $\mathrm{N}$ methoxypyridinium salt 2a was prepared by dissolving 2a $(30 \mu \mathrm{mol})$ in $10 \mathrm{~mL}$ of $\mathrm{MeCN}$. Concentration of $[\mathbf{2 a}]=3 \times 10^{-3} \mathrm{M}$. A stock solution of $\mathbf{1 a}$ was prepared by dissolving $1 \mathbf{a}$ (30 $\mu \mathrm{mol}$ ) in $10 \mathrm{~mL}$ of $\mathrm{MeCN}$. Concentration of $[\mathbf{1 a}]=3 \times 10^{-3} \mathrm{M}$. For each experiment, 6 samples were prepared in the dark. Quartz cuvettes $(3.5 \mathrm{~mL})$ were filled with photocatalyst stock solution $(0.3 \mathrm{~mL})$, reagent stock solution $(0 \mathrm{~mL}, 0.05 \mathrm{~mL}, 0.1 \mathrm{~mL}, 0.15 \mathrm{~mL}, 0.2 \mathrm{~mL}$ et 0.25 $\mathrm{mL}$ ) and $\mathrm{MeCN}(2.7 \mathrm{~mL}, 2.65 \mathrm{~mL}, 2.6 \mathrm{~mL}, 2.55 \mathrm{~mL}, 2.5 \mathrm{~mL}, 2.45 \mathrm{~mL})$ to obtain a total volume of $3 \mathrm{~mL}$. The final concentrations were $[3 \mathbf{d}]=2.510^{-6} \mathrm{M}$ and [quencher] $=5.10^{-5} \mathrm{M}, 1.10^{-4} \mathrm{M}$, 1.5.10 $10^{-4} \mathrm{M}, 2.10^{-4} \mathrm{M}, 2.5 .10^{-3} \mathrm{M}$. For each sample, emission spectra were acquired between 450 $\mathrm{nm}$ and $650 \mathrm{~nm}$ (excitation at $450 \mathrm{~nm}$ ).

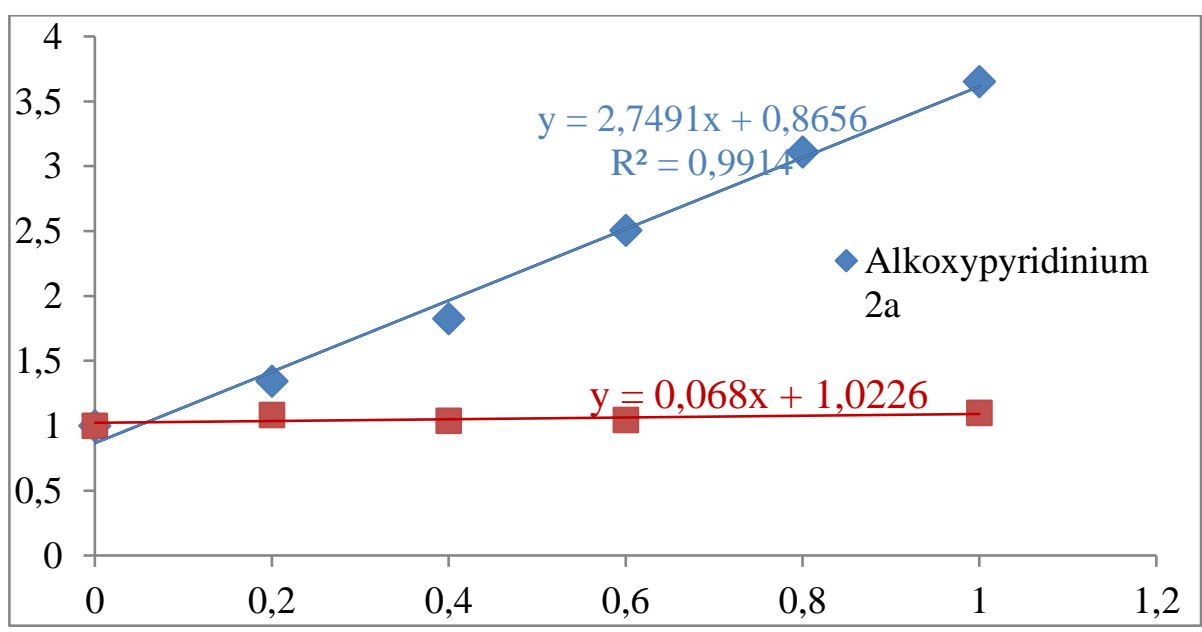

Figure S1: Stern-Volmer plots for N-methoxypyridinium 2a and silyl enol ether 1a as quenchers 


\section{Light On/Off experiment}

Silylated enol ether 1a (0.20 mmol, 1 equiv.), $\mathrm{K}_{2} \mathrm{HPO}_{4}(69.7 \mathrm{mg}, 0.40 \mathrm{mmol}, 2$ equiv.), $\mathrm{Ru}(\mathrm{bpy})_{3}\left(\mathrm{PF}_{6}\right)_{2}$ 3d $(1.7 \mathrm{mg}, 0.002 \mathrm{mmol}, 1 \mathrm{~mol} \%)$, and $\mathrm{N}$-methoxypyridinium salt 2a ( 0.36 mmol, 1.8 equiv.) are introduced in a dry tube. Then, $2 \mathrm{~mL}$ of dry $\mathrm{MeCN}$ are added and the solution is degazed under argon and stirred at room temperature under blue LED irradiation $(7.5 \mathrm{~W}, 450 \mathrm{~nm})$ or under dark (aluminum foil) during the indicated time. Yield is calculated by ${ }^{19} \mathrm{~F}$ NMR using PhF as internal standard.

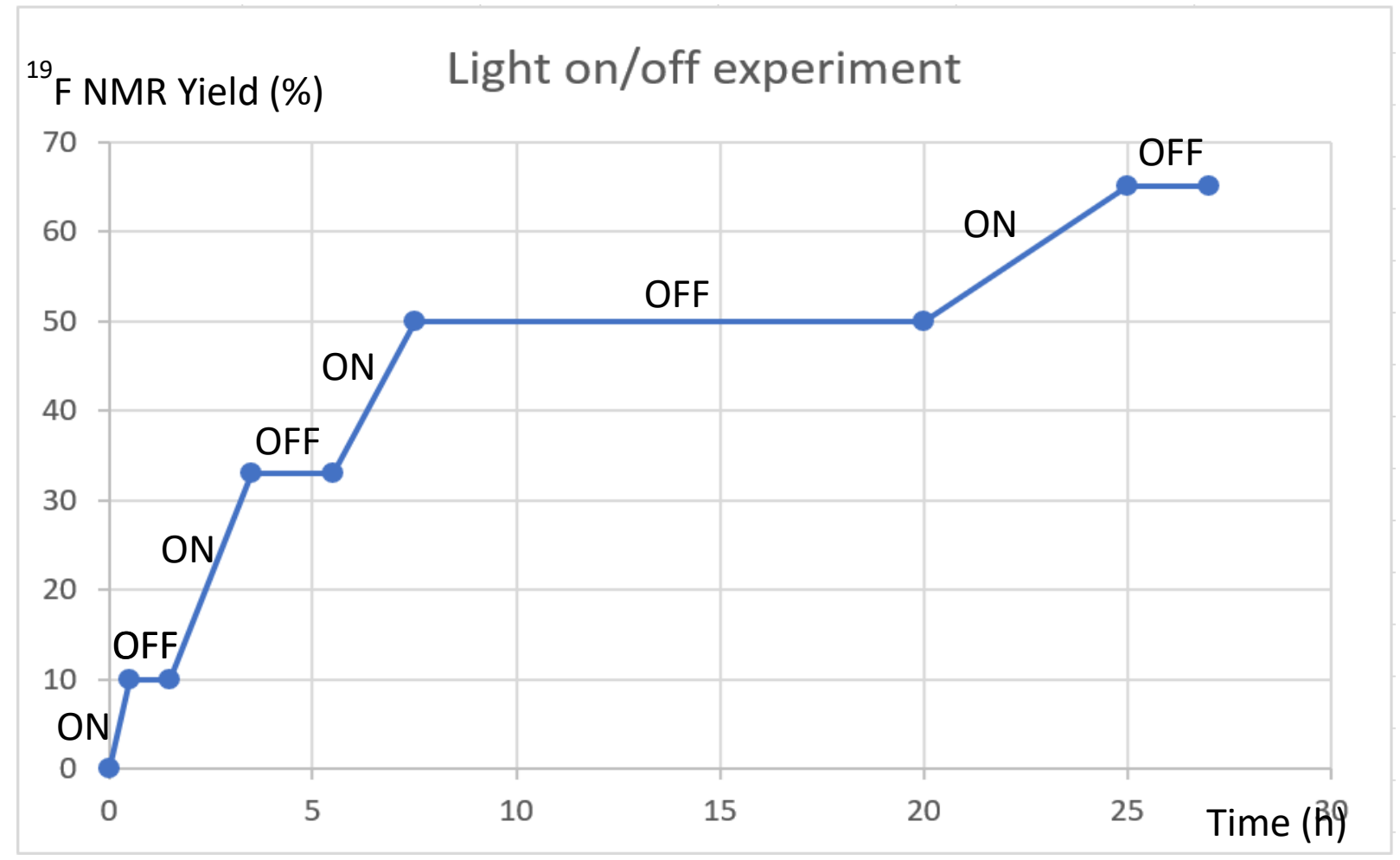




\section{Quantum yield measurement}

The photon flux was determined by standard ferrioxalate actinometry. ${ }^{[7]}$

\section{Solutions needed:}

$0.05 \mathrm{M}$ sulfuric acid stock solution:

In a $100 \mathrm{~mL}$ volumetric flask, $0.281 \mathrm{~mL}$ of concentrated sulfuric acid $(17.8 \mathrm{M})$ was added to $90 \mathrm{~mL}$ deionized water. Then, water was added until the $100 \mathrm{~mL}$ graduation mark was reached.

\section{Ferrioxolate solution:}

A $0.15 \mathrm{M}$ solution of potassium ferrioxolate was prepared in a $25 \mathrm{~mL}$ volumetric flask by dissolving potassium ferrioxolate $\left(\mathrm{K}_{3} \mathrm{FeC}_{2} \mathrm{O}_{4} * 3 \mathrm{H}_{2} \mathrm{O}\right)(1.842 \mathrm{~g}, 3.75 \mathrm{mmol})$ with the $0.05 \mathrm{M}$ sulfuric acid solution. The solution was prepared and in the dark.

\section{Developer solution:}

$22.5 \mathrm{~g}$ of sodium acetate trihydrate was dissolved in $100 \mathrm{~mL}$ of $0.5 \mathrm{M}$ sulfuric acid. $1 \mathrm{~g}$ of 1,10phenantroline was added to this solution. Store the solution in the dark.

\section{Typical Experiment carried out under dark:}

$200 \mu \mathrm{L}$ of $0.15 \mathrm{M}$ aqueous potassium ferrioxalate was transferred to a $5 \mathrm{~mm}$ thin wall NMR tube followed by the placement of the coaxial insert. Then the sample was irradiated with 448 $\mathrm{nm}$ LED at room temperature. The procedure was repeated with different irradiations times for different samples.

$100 \mu \mathrm{L}$ aliquots of the solution were taken from each solution and added immediately to $3 \mathrm{~mL}$ of a developer solution of sodium acetate and 1,10-phenanthroline and the flask was quickly wrapped in aluminum foil. Concurrently, a "blank" sample was prepared by diluting $100 \mu \mathrm{L}$ of the stock solution (kept in the dark) into $3 \mathrm{~mL}$ of developer solution. The solutions were left in the dark for $30 \mathrm{~min}-1 \mathrm{hr}$, becoming bright red. The solutions were transferred to a cuvette and the absorbance spectrum of the $\mathrm{Fe}(\text { phen })_{3}{ }^{2+}$ complex was obtained. The absorbance at $510 \mathrm{~nm}$ $\left(\varepsilon=11,100 \mathrm{M}^{-1} \cdot \mathrm{cm}^{-1}\right)$ was measured for every sample.

\section{Data analysis:}

To calculate photon flux from your chemical actinometry data, first determine the number of $\mathrm{Fe}^{2+}$ ions produced by ferrioxolate photo-degradation:

$$
\text { moles } \mathrm{Fe}^{2+}=\frac{\Delta A_{510 \mathrm{~nm}} V_{1} V_{3}}{\varepsilon_{510 \mathrm{~nm}} l V_{2}}
$$

$\Delta A=$ difference in absorbance at $510 \mathrm{~nm}$ between sample and 'blank' 
$1=$ path length of cuvette $(1 \mathrm{~cm})$

$\varepsilon=$ Extinction coefficient of $\mathrm{Fe}(\text { phen })_{3}$ complex at $510 \mathrm{~nm}\left(\varepsilon=11,100 \mathrm{M}^{-1} \cdot \mathrm{cm}^{-1}\right)$

$\mathrm{V}_{1}=$ total volume of irradiated solution $(200 \mu \mathrm{L})$

$\mathrm{V}_{2}=$ volume of aliquot taken from $\mathrm{V}_{1}(100 \mu \mathrm{L})$

$\mathrm{V}_{3}=$ the volume that $\mathrm{V}_{2}$ is diluted into $(3 \mathrm{~mL})$

The photon flux can be determined:

$$
\text { photon flux }=\frac{\text { moles of } \mathrm{Fe}^{2+}}{\Phi_{4 \mathrm{ssm}} \times t \times F}
$$

with:

$\mathrm{F}_{448 \mathrm{~nm}}=1.01$ (reported literature value) $^{[8]}$

$\mathrm{t}=$ time of irradiation (seconds)

$\mathrm{F}=$ mean fraction of light absorbed by the ferrioxalate solution $(\mathrm{F} \approx 1$ at $448 \mathrm{~nm}$ at $0.15 \mathrm{M}$ ferrioxolate).

The linear dependence of $\mathrm{Fe}^{2+}$ accumulation on LED irradiation time at $448 \mathrm{~nm}$ is plotted:

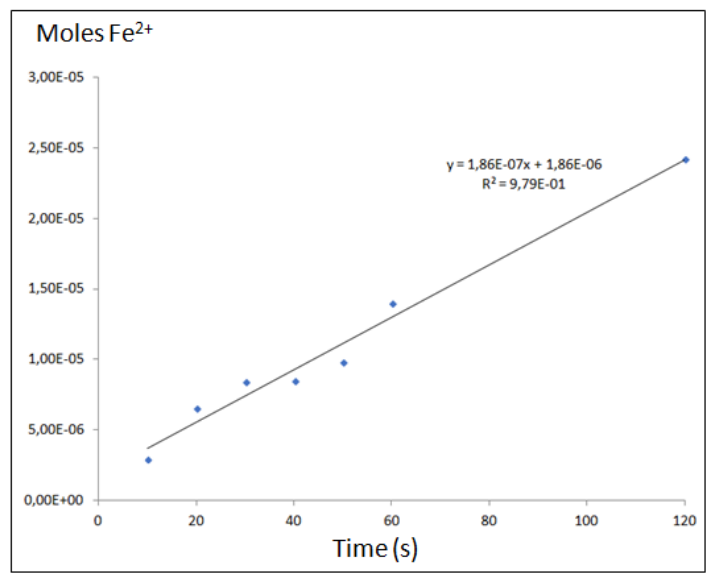

Figure S2: linear dependence of $\mathrm{Fe}^{2+}$ accumulation on LED irradiation time at $448 \mathrm{~nm}$

From the slope of the linear regression line, we finally find the photon flux:

$$
\text { Photon flux }=1.86 .10^{-7} \text { mol.s }^{-1}
$$

\section{Quantum yield measurement}

$$
\Phi=(\text { rate of substrate conversion }) /(\text { absorbed photon flux })
$$

The rate of substrate conversion was measured by analyzing the reaction mixture as a function of time thanks to in situ NMR irradiation. 
General procedure: A solution of N-methoxypyridinium salt $\mathbf{2 a}(0.36 \mathrm{mmol})$, silyl enol ether $1 \mathbf{a}(0.20 \mathrm{mmol}), \mathrm{K}_{2} \mathrm{HPO}_{4}(0.40 \mathrm{mmol})$ and the photocatalyst $\mathbf{3 d}(0.002 \mathrm{mmol})$ in $\mathrm{CD}_{3} \mathrm{CN}(2 \mathrm{~mL})$ was prepared and $0.25 \mathrm{~mL}$ of this solution was introduced in an NMR tube and the mixture was degassed with argon.

Excitation was performed at $448 \mathrm{~nm}$ and substrate conversion was periodically determined (after 60 seconds, then each 180 seconds) by comparison of the integration of ${ }^{19} \mathrm{~F}$ NMR of silyl enol ether 1a peak and fluorobenzene peak, used as internal standard (Brucker AC-300, operating at $282 \mathrm{MHz}$ for ${ }^{19} \mathrm{~F} \mathrm{NMR}$ ).

The substrate conversion (as well as the product formation) was then plotted against time (see Figure S3):

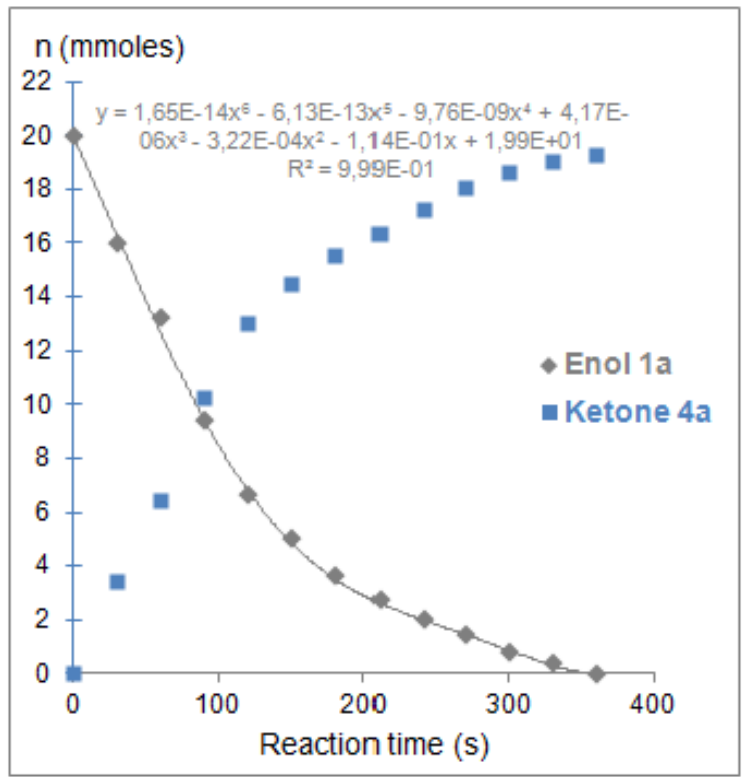

Figure S3: Quantity of starting material 1a and product $4 \mathbf{a}$ versus time. The plot is fitted to a 6th order polynomial

The derivative of the polynomial of Figure S3 is used to calculate the rate of substrate conversion.

Then the absorbed photon flux has to be calculated. Note that in the case of NMR experiments with an optical fiber, the path length of irradiated solution is very small $(0.06 \mathrm{~cm})$ and the fraction (f) of light absorbed by this solution has to be first calculated using the equation below, where $\mathrm{A}$ is the measured absorbance at $448 \mathrm{~nm}$.

$$
\mathbf{f}=\mathbf{1}-\mathbf{1 0}^{-\mathbf{A}}
$$

with $\mathrm{A}=\varepsilon_{448 \mathrm{~nm} .1 .[\mathrm{Ru}]}$

The molar absorptivity $\varepsilon_{448 \mathrm{~nm}}$ is $13000 \mathrm{M}^{-1} \cdot \mathrm{cm}^{-1} \cdot{ }^{[9]}$

Thus, the fraction $\mathrm{f}$ of light absorbed was calculated:

$$
\mathbf{f}=\mathbf{0 . 8 3}
$$


Then, absorbed photon flux $=$ photon flux $\bullet \mathrm{f}=\mathbf{1 . 5 4 . 1 0 ^ { - 7 }} \mathbf{m o l}^{\mathbf{- 1}}$.

Finally, the quantum yield of the reaction can be calculated. The initial value is $\mathbf{\Phi = \mathbf { 0 . 7 4 }}$ and it decreases when the concentration of substrate 1a decreases. 


\section{UV/Vis experiments}

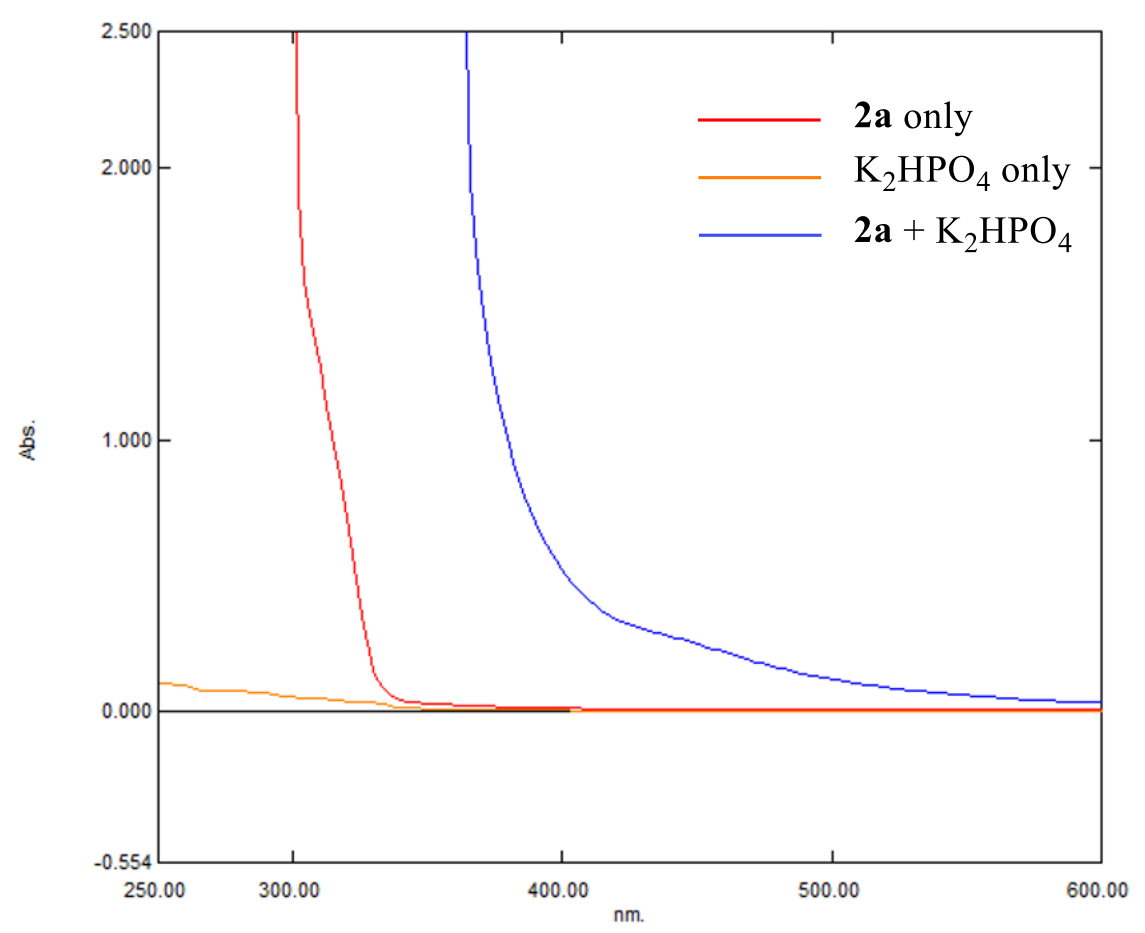

Uv-vis spectra of a solution of a solution $N$-methoxypyridinium $2 \mathbf{a}$ (red curve, $5.10^{-2} \mathrm{M}$ in a $1: 1$ mixture of $\mathrm{CH}_{2} \mathrm{Cl}_{2} / \mathrm{MeCN}$ ), $\mathrm{K}_{2} \mathrm{HPO}_{4}$ (orange curve, $5.10^{-2} \mathrm{M}$ in a $1: 1$ mixture of $\mathrm{CH}_{2} \mathrm{Cl}_{2} / \mathrm{MeCN}$ ), and a mixture of $2 \mathrm{a}+\mathrm{K}_{2} \mathrm{HPO}_{4}$ (blue curve, $5.10^{-2} \mathrm{M}$ in a $1: 1$ mixture of $\left.\mathrm{CH}_{2} \mathrm{Cl}_{2} / \mathrm{MeCN}\right)$.

Based on these spectra, an EDA complex was formed between $\mathrm{K}_{2} \mathrm{HPO}_{4}$ and $\mathbf{2 a}$. 
VIII. NMR spectra of new compounds

1-(4-fluorophenyl)-2-methoxyethanone 4a

${ }^{1} \mathrm{H}$ NMR (300 MHz, $\left.\mathrm{CDCl}_{3}\right)$

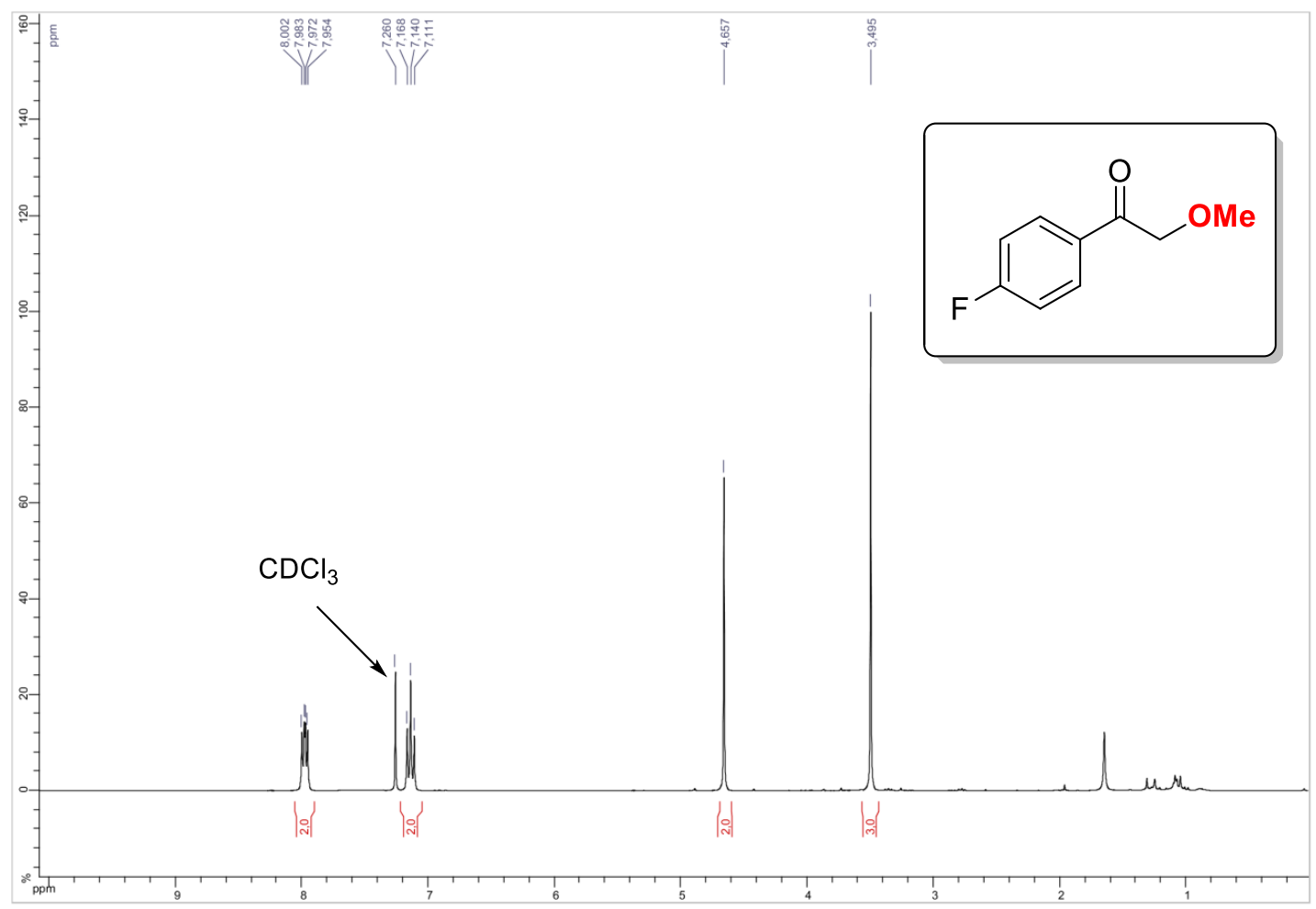


${ }^{13} \mathrm{C}$ NMR (75 MHz, $\left.\mathrm{CDCl}_{3}\right)$

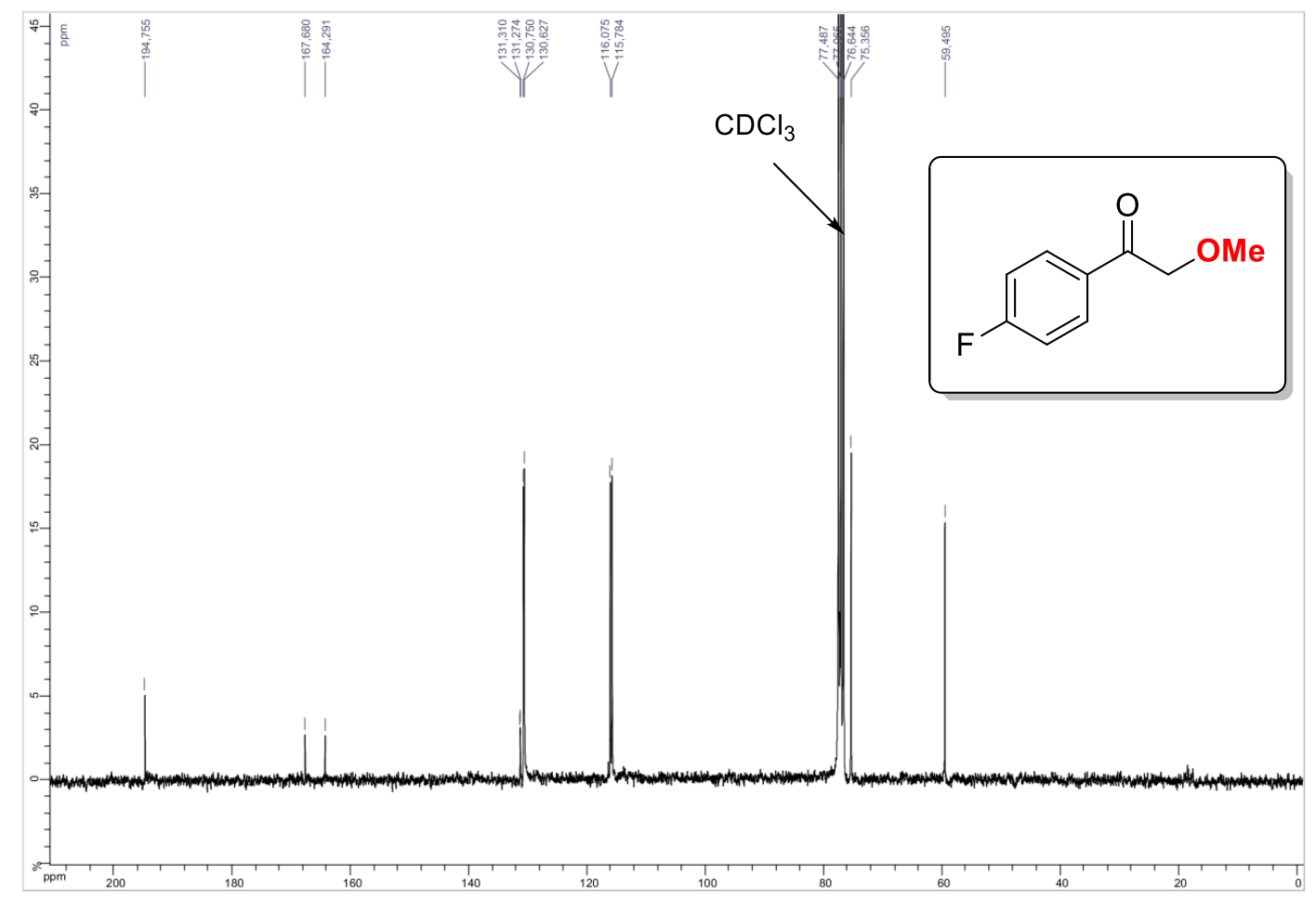

${ }^{19}$ F NMR (282 MHz, $\left.\mathrm{CDCl}_{3}\right)$

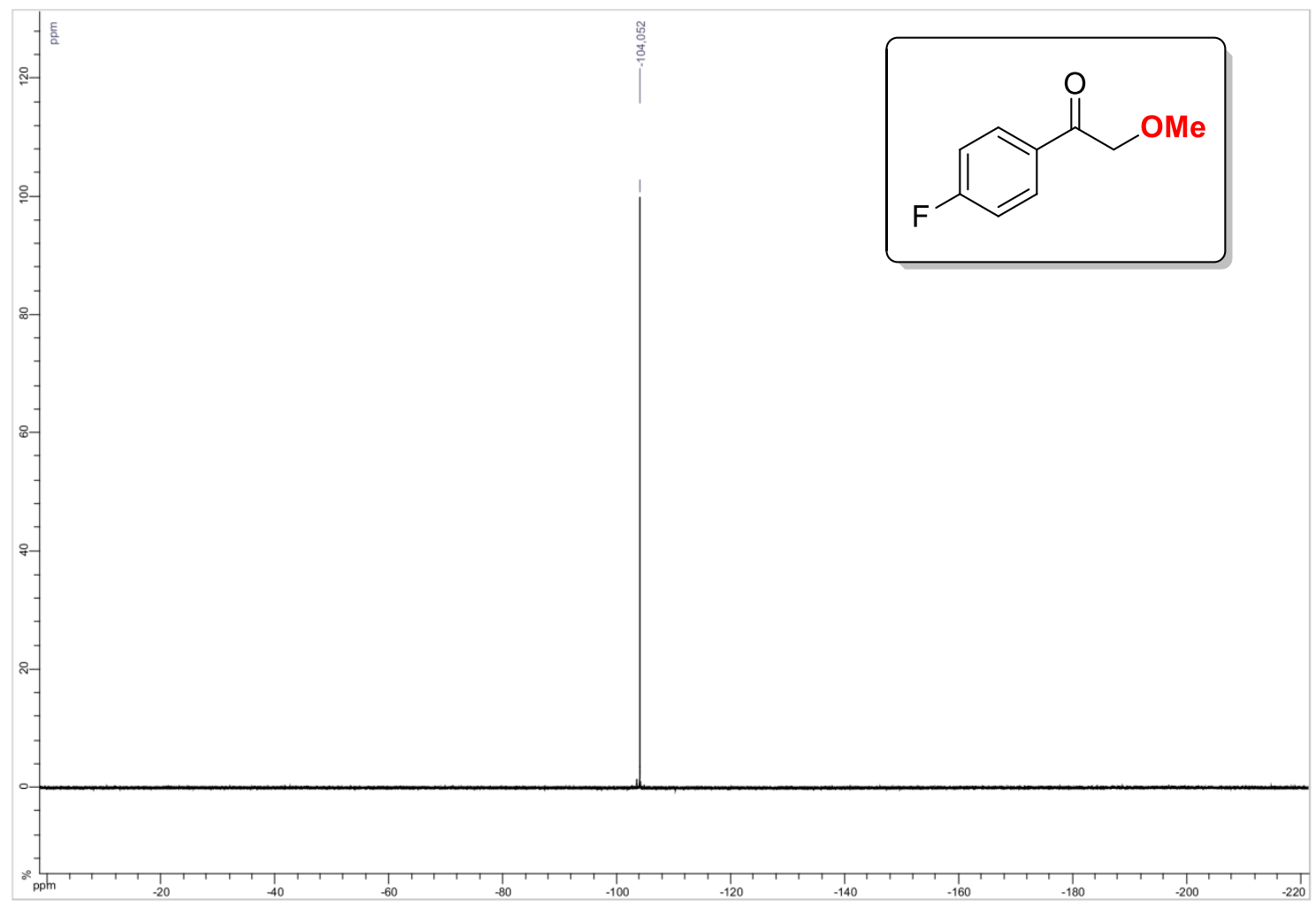


2-Methoxy-1-(4-methoxyphenyl)ethanone 4b

${ }^{1} \mathrm{H}$ NMR (300 MHz, $\left.\mathrm{CDCl}_{3}\right)$

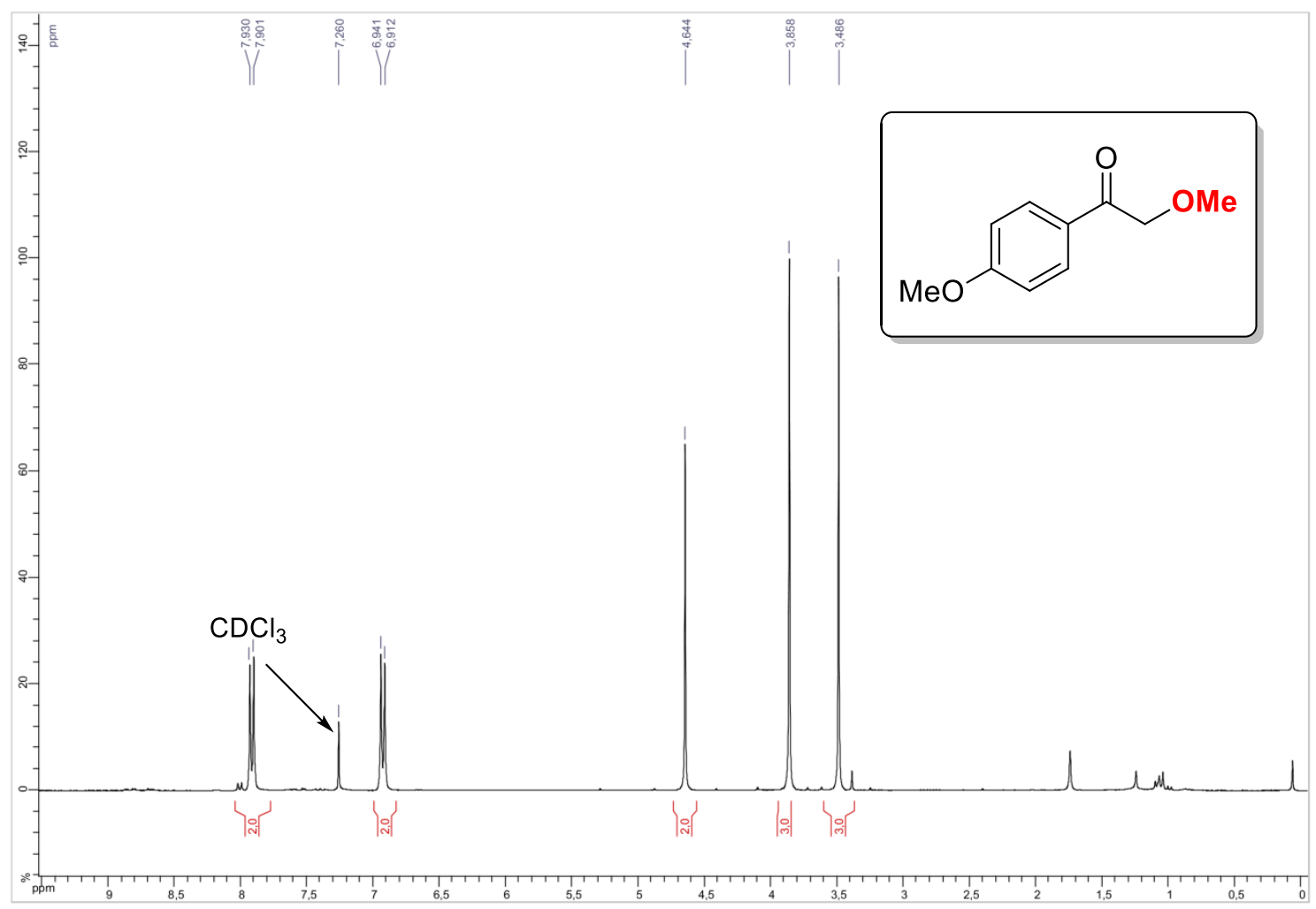

${ }^{13} \mathrm{C}$ NMR (75 MHz, CDCl3)

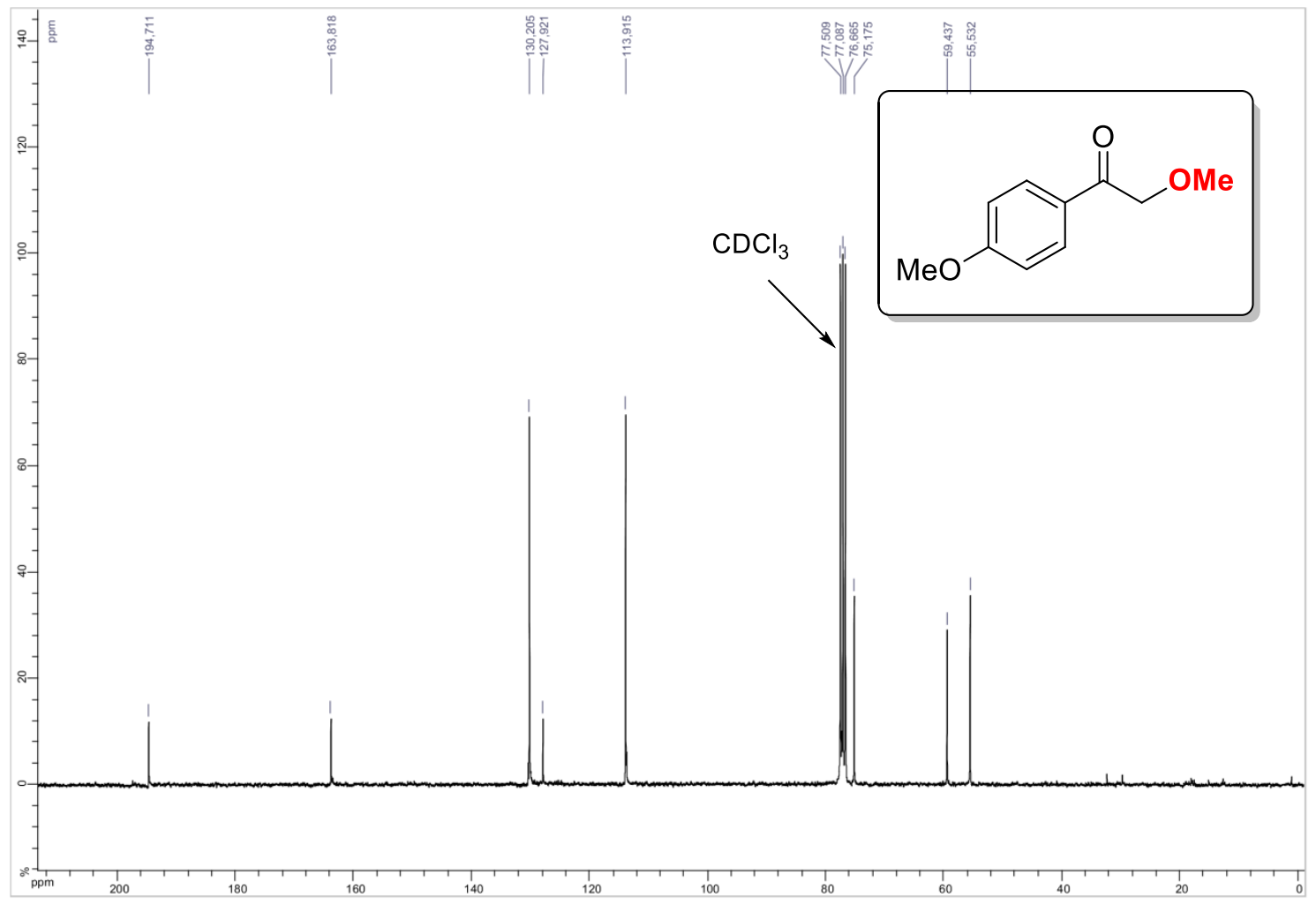


2-Methoxy-1-(p-tolyl)ethanone 4c

${ }^{1}$ H NMR (300 MHz, CDCl3)

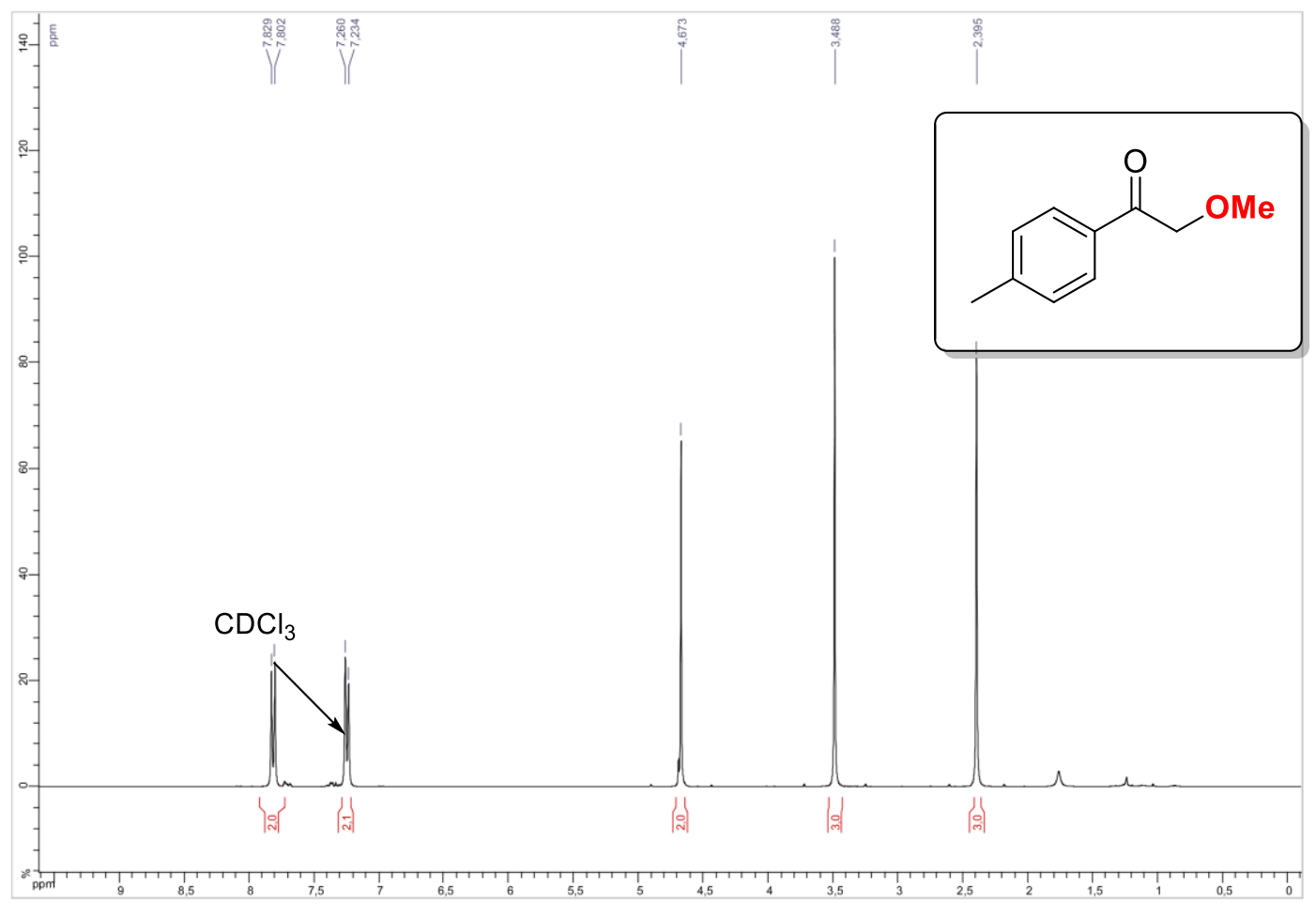

${ }^{13} \mathrm{C}$ NMR (75 MHz, CDCl3)

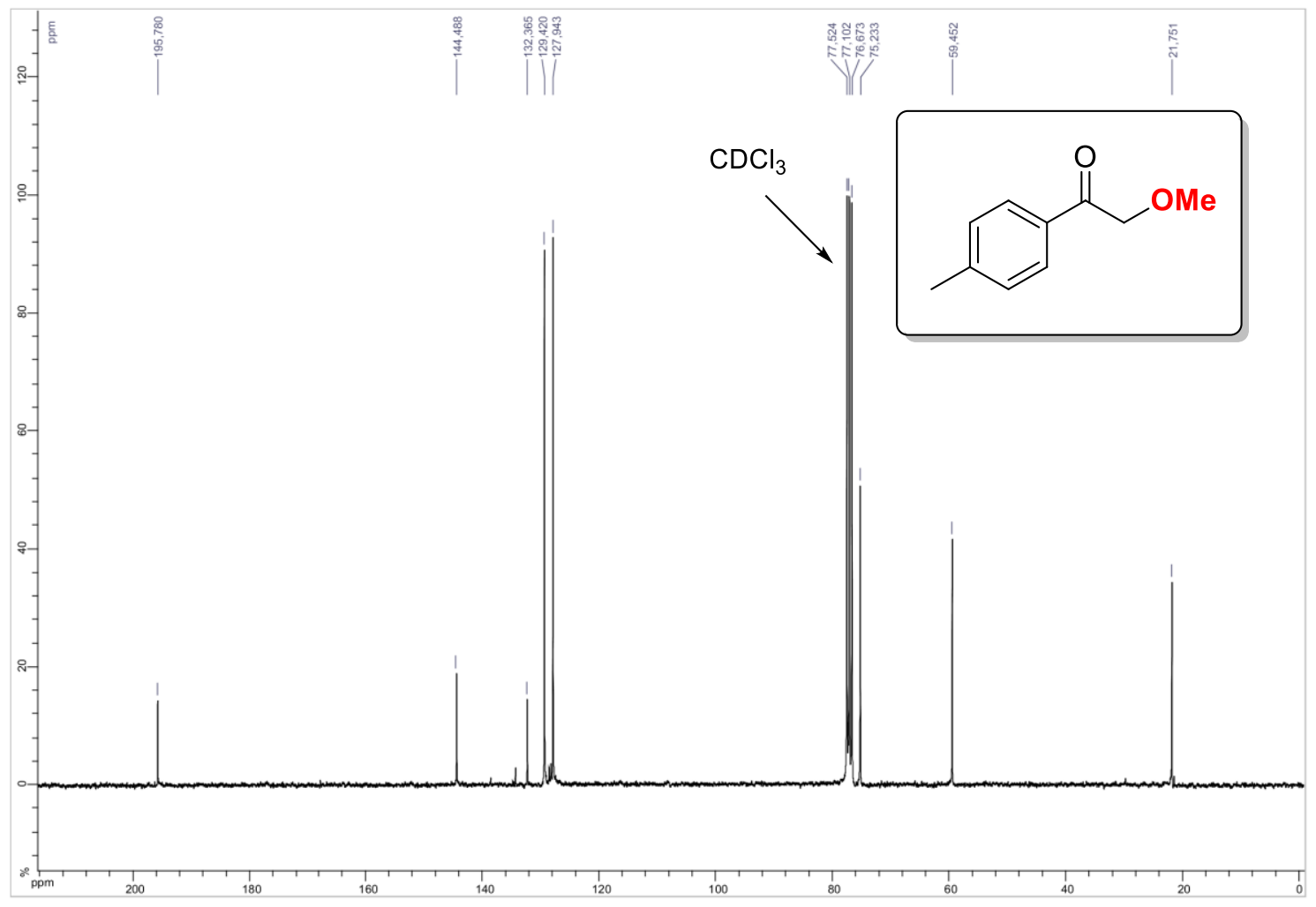


1-(4-Chlorophenyl)-2-methoxyethanone 4d

${ }^{1} \mathrm{H}$ NMR (300 MHz, CDCl 3 )

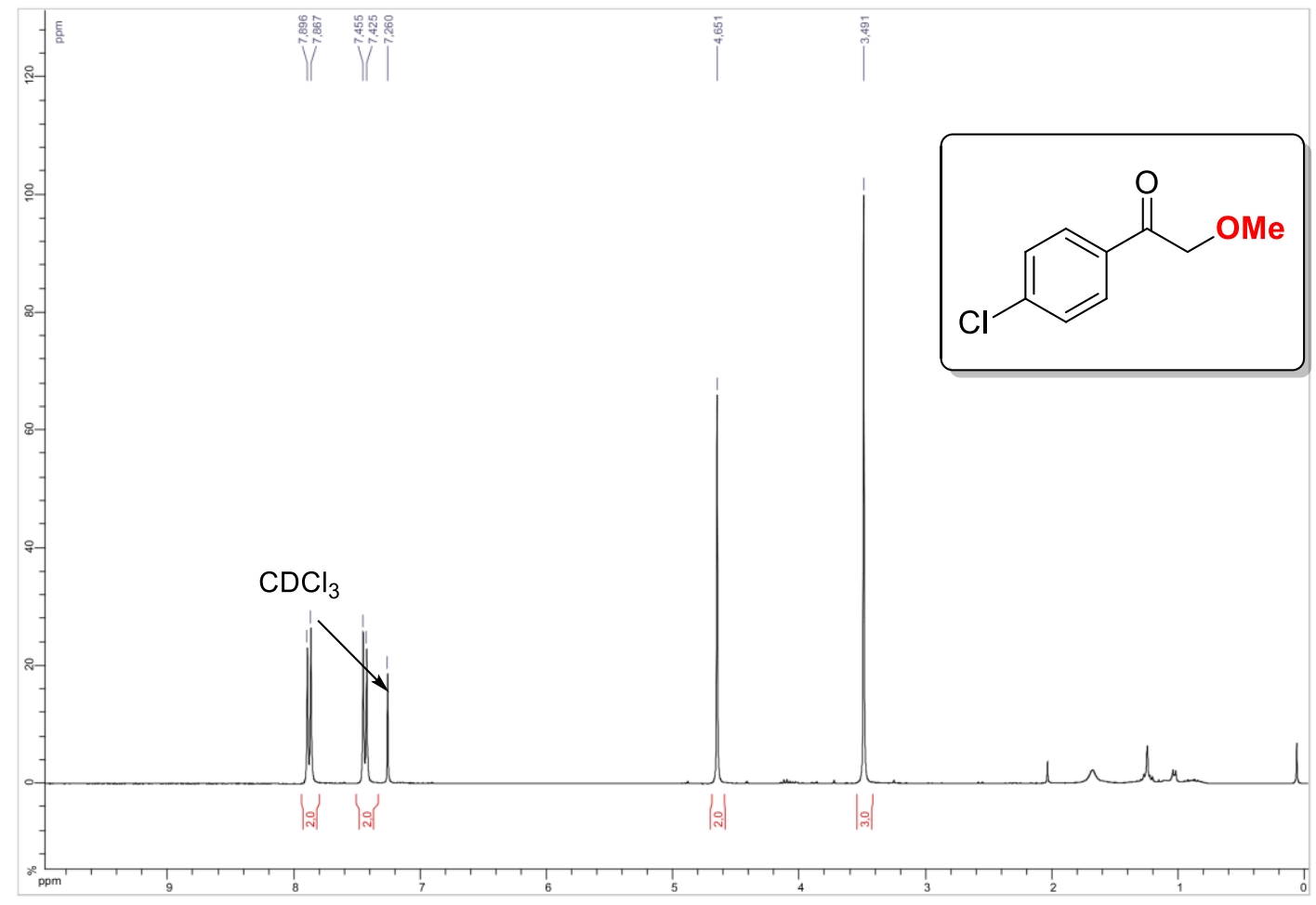

${ }^{13} \mathrm{C}$ NMR (75 MHz, $\left.\mathrm{CDCl}_{3}\right)$

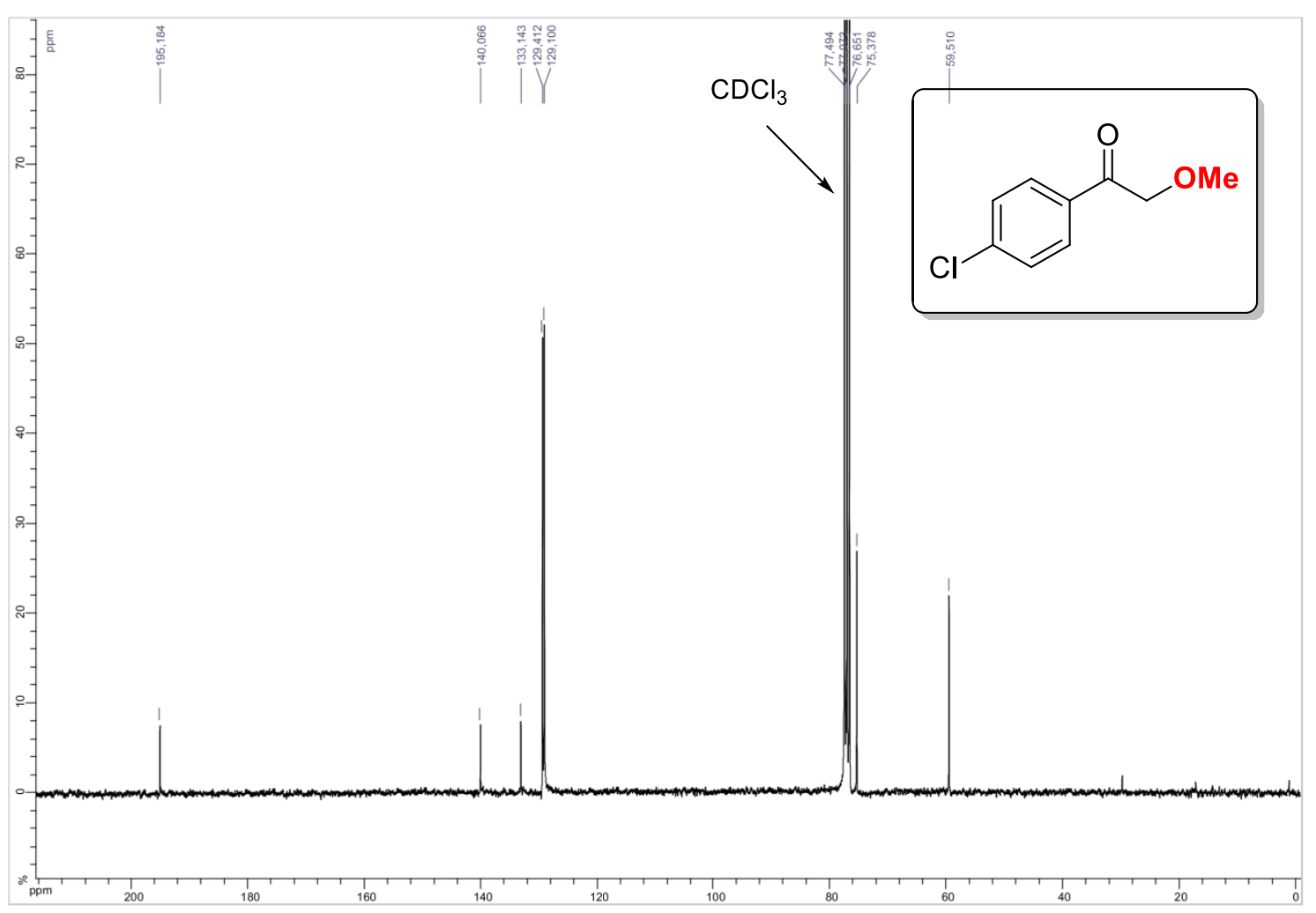


1-(4-Bromophenyl)-2-methoxyethanone 4e

${ }^{1} \mathrm{H}$ NMR (300 MHz, CDCl 3 )

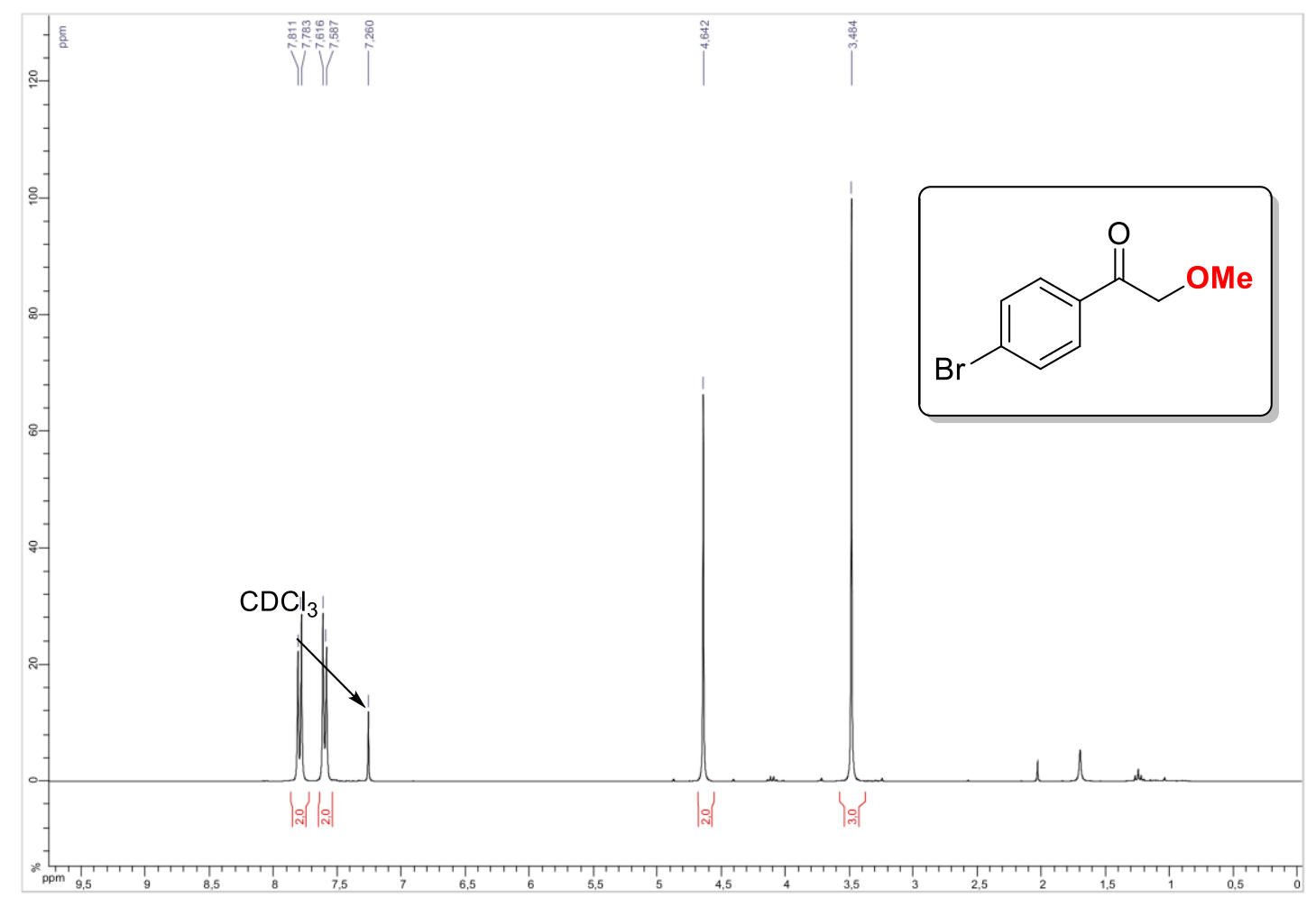

${ }^{13} \mathrm{C}$ NMR (75 MHz, CDCl3)

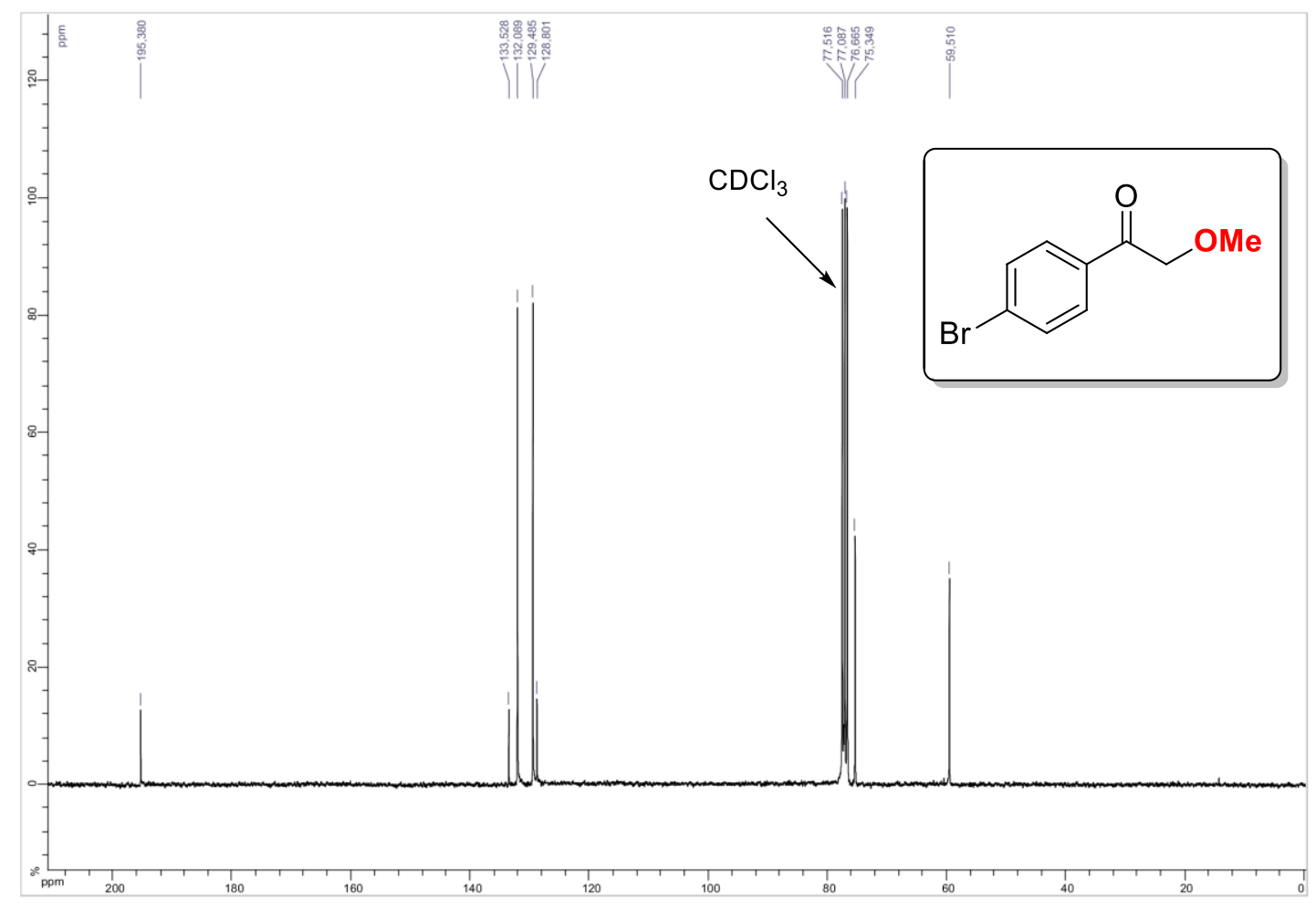


2-Methoxy-1-(o-tolyl)ethanone 4 f

${ }^{1} \mathrm{H}$ NMR (300 MHz, $\left.\mathrm{CDCl}_{3}\right)$

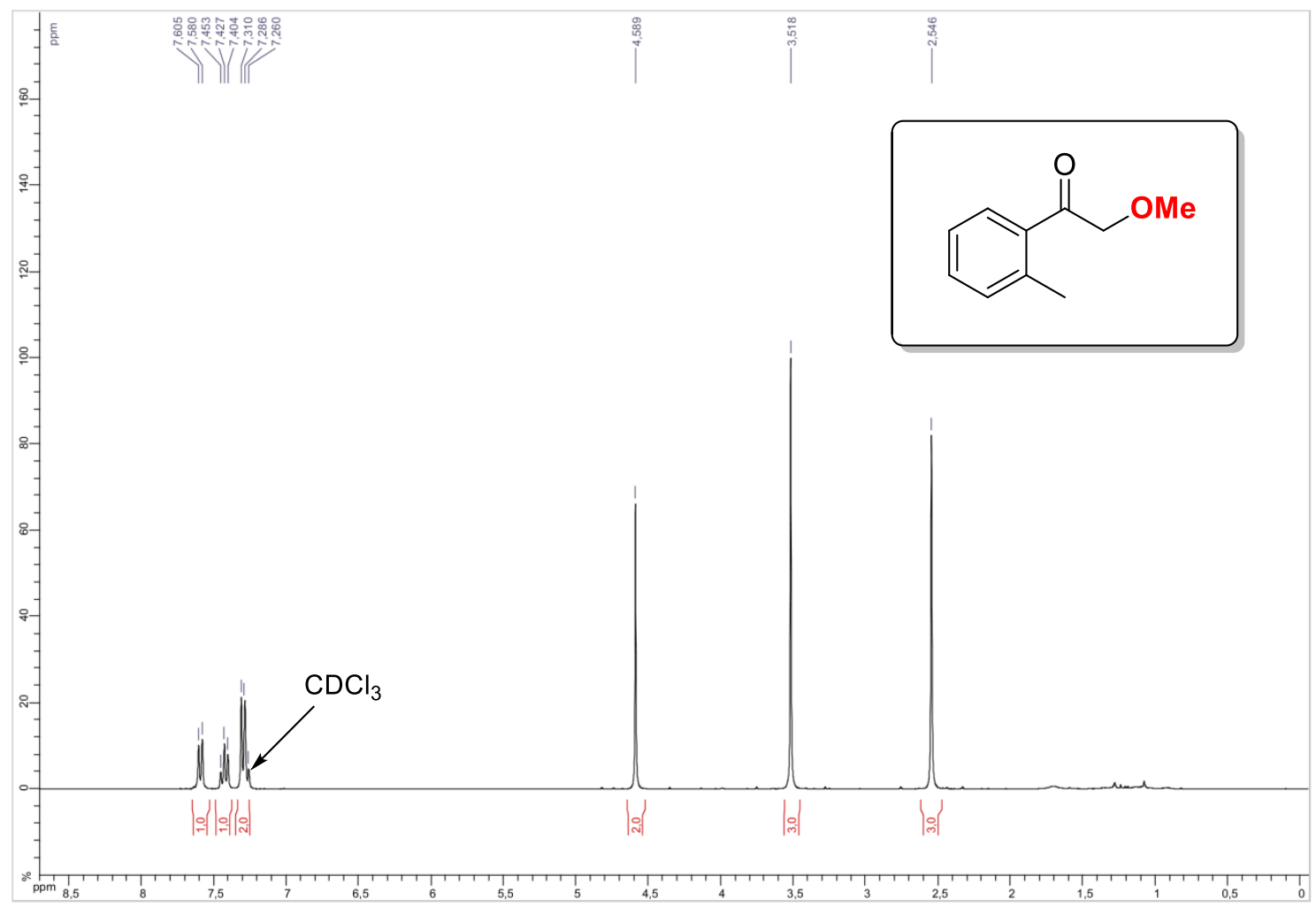

${ }^{13} \mathrm{C}$ NMR (75 MHz, CDCl3)

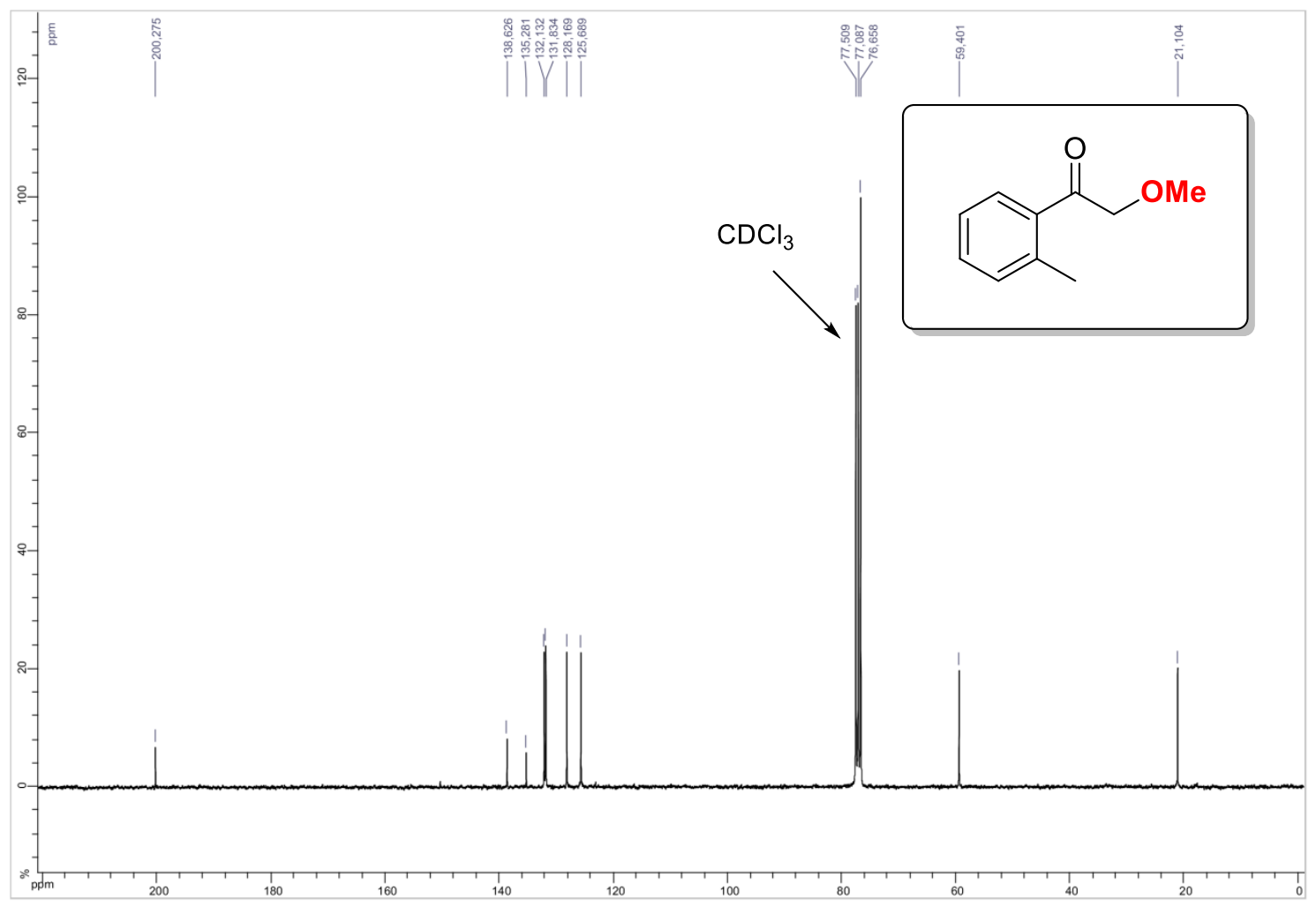


2-Methoxy-1-phenylethanone 4g

${ }^{1} \mathrm{H}$ NMR (300 MHz, $\left.\mathrm{CDCl}_{3}\right)$

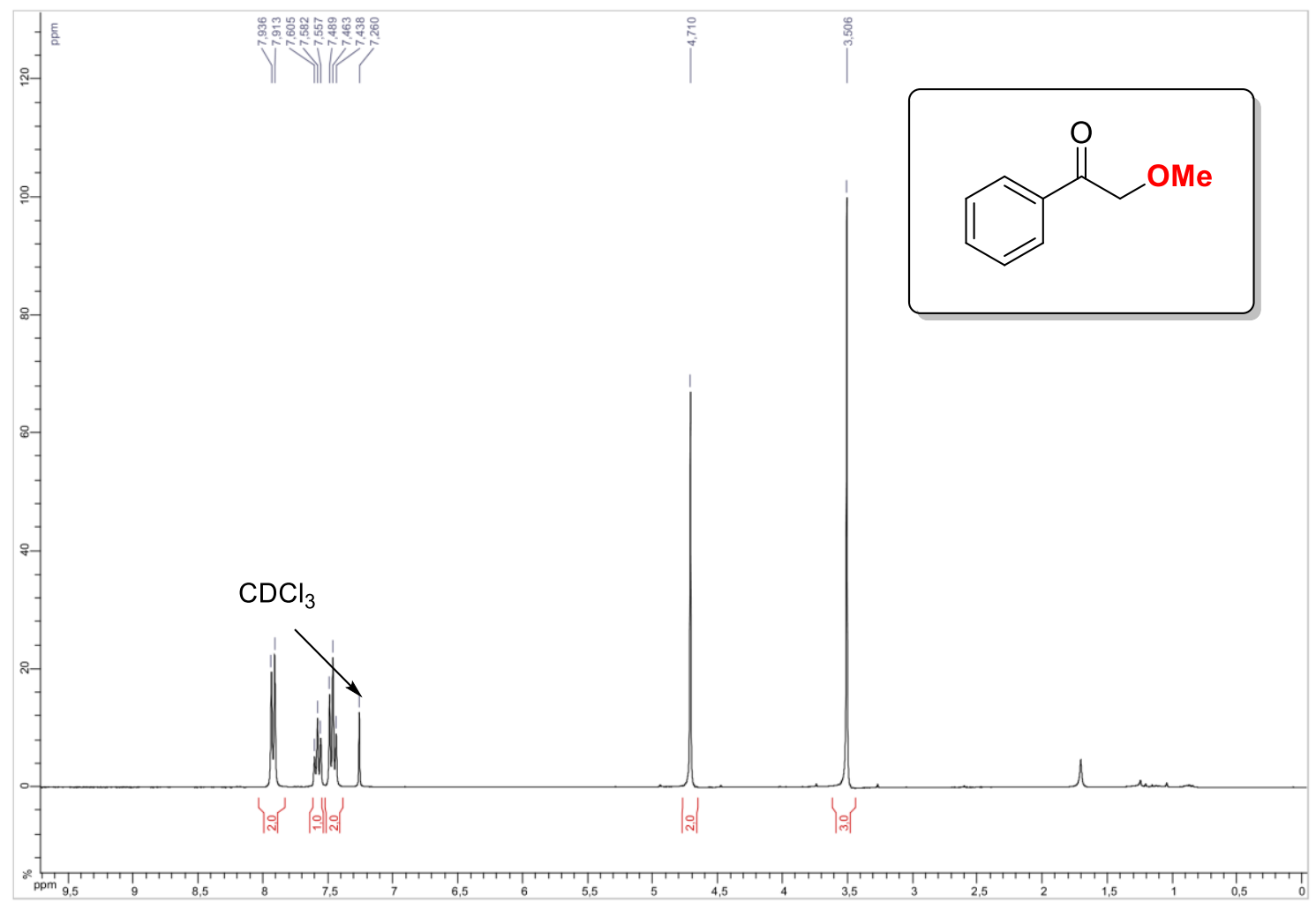

${ }^{13} \mathrm{C}$ NMR (75 MHz, CDCl3)

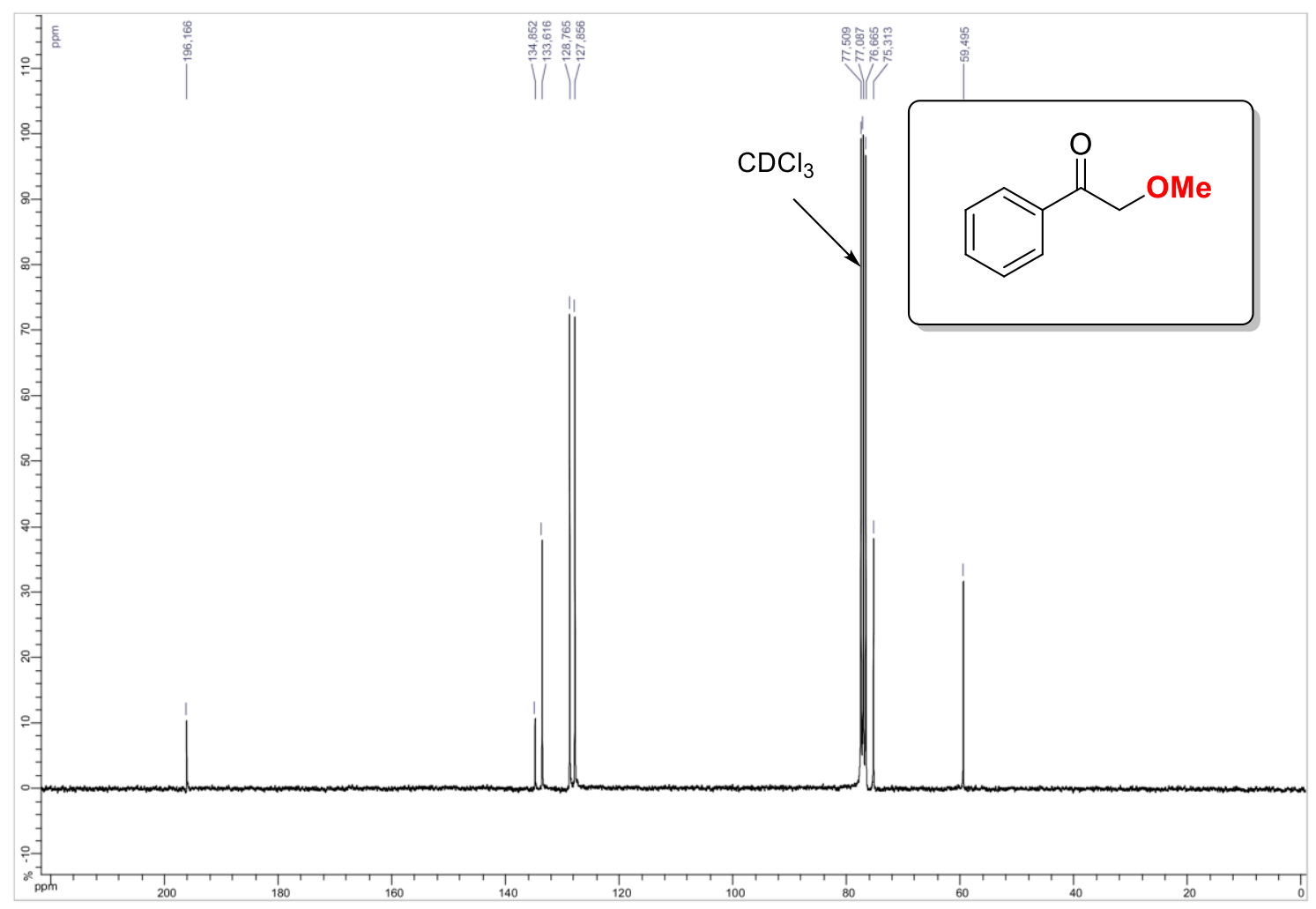


Methyl 3-(2-methoxyacetyl)benzoate 4h

${ }^{1} \mathrm{H}$ NMR (300 MHz, $\left.\mathrm{CDCl}_{3}\right)$

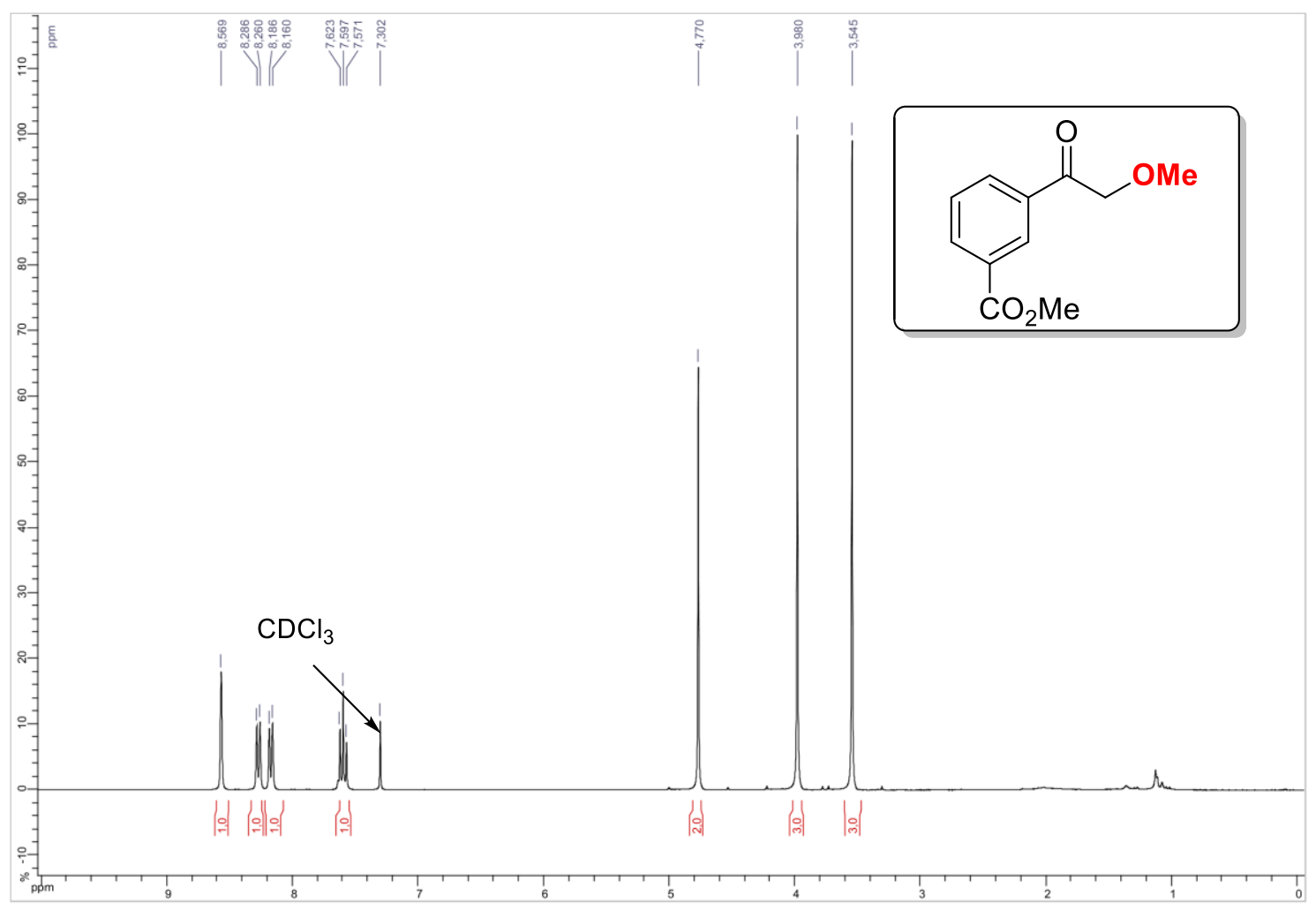

${ }^{13} \mathrm{C}$ NMR (75 MHz, CDCl3)

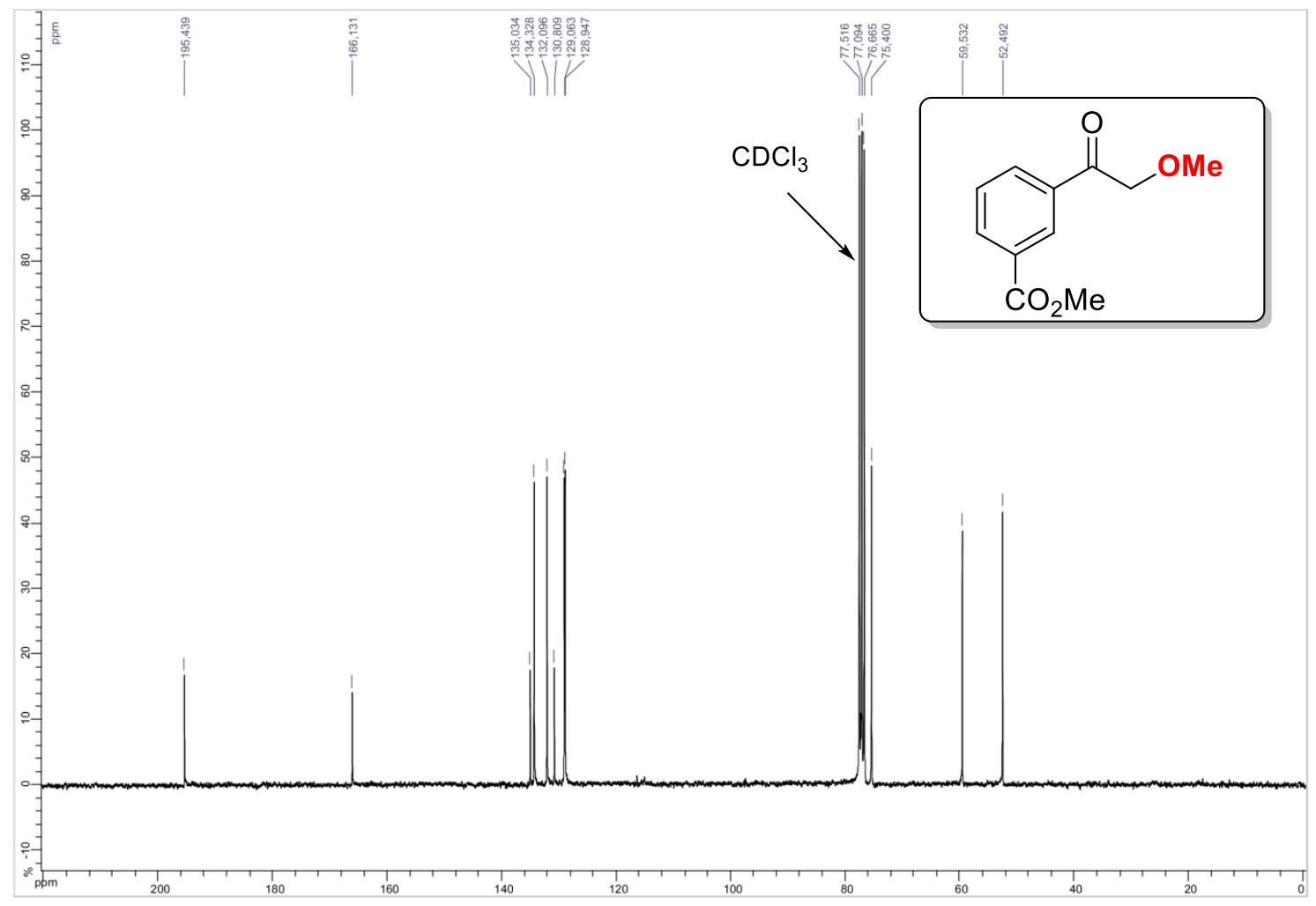


2-Methoxy-1-(3-(trifluoromethyl)phenyl)ethanone 4i

${ }^{1} \mathrm{H}$ NMR (300 MHz, $\left.\mathrm{CDCl}_{3}\right)$

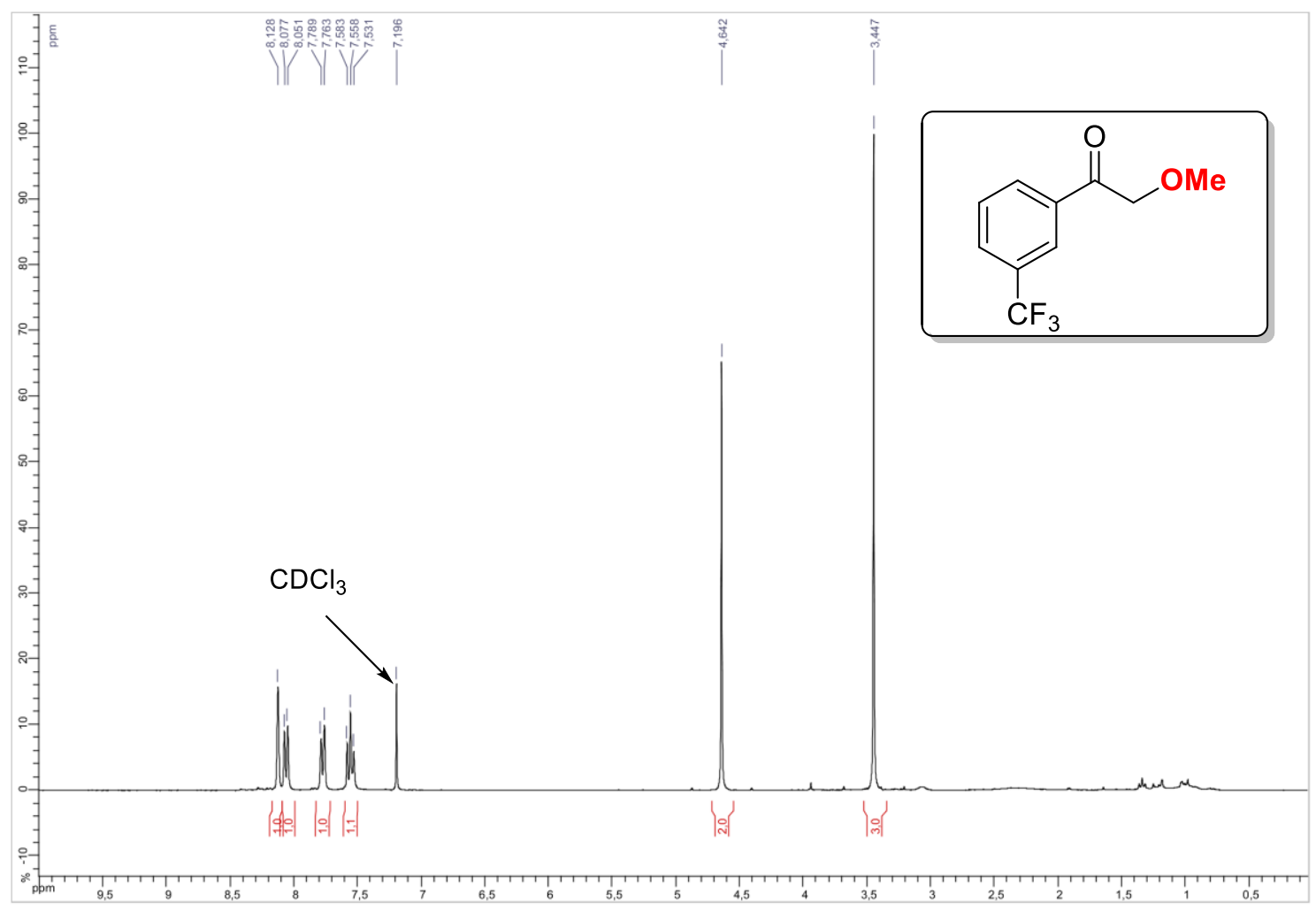

${ }^{13} \mathrm{C}$ NMR (75 MHz, CDCl3)

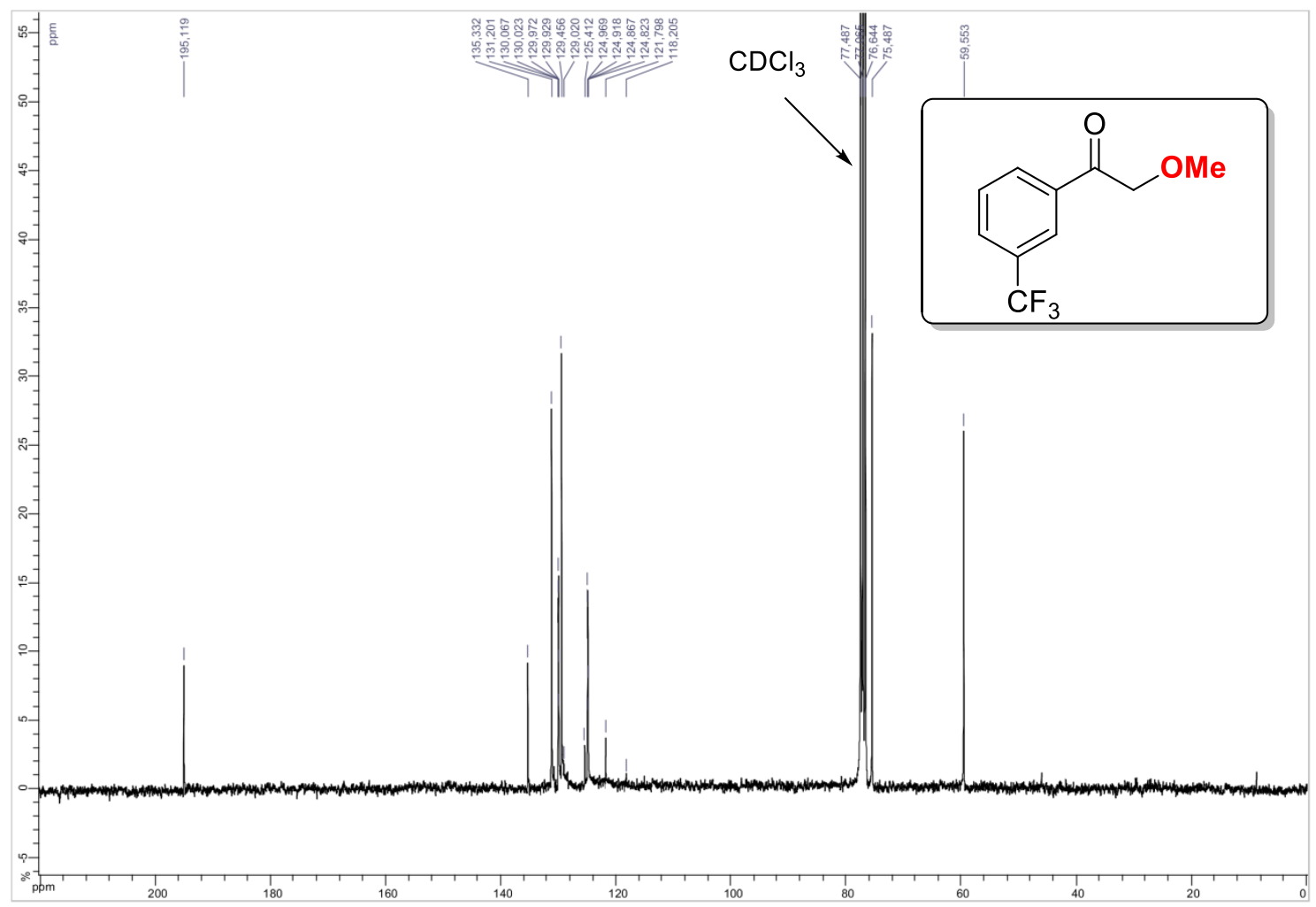


${ }^{19}$ F NMR (282 MHz, CDCl 3$)$

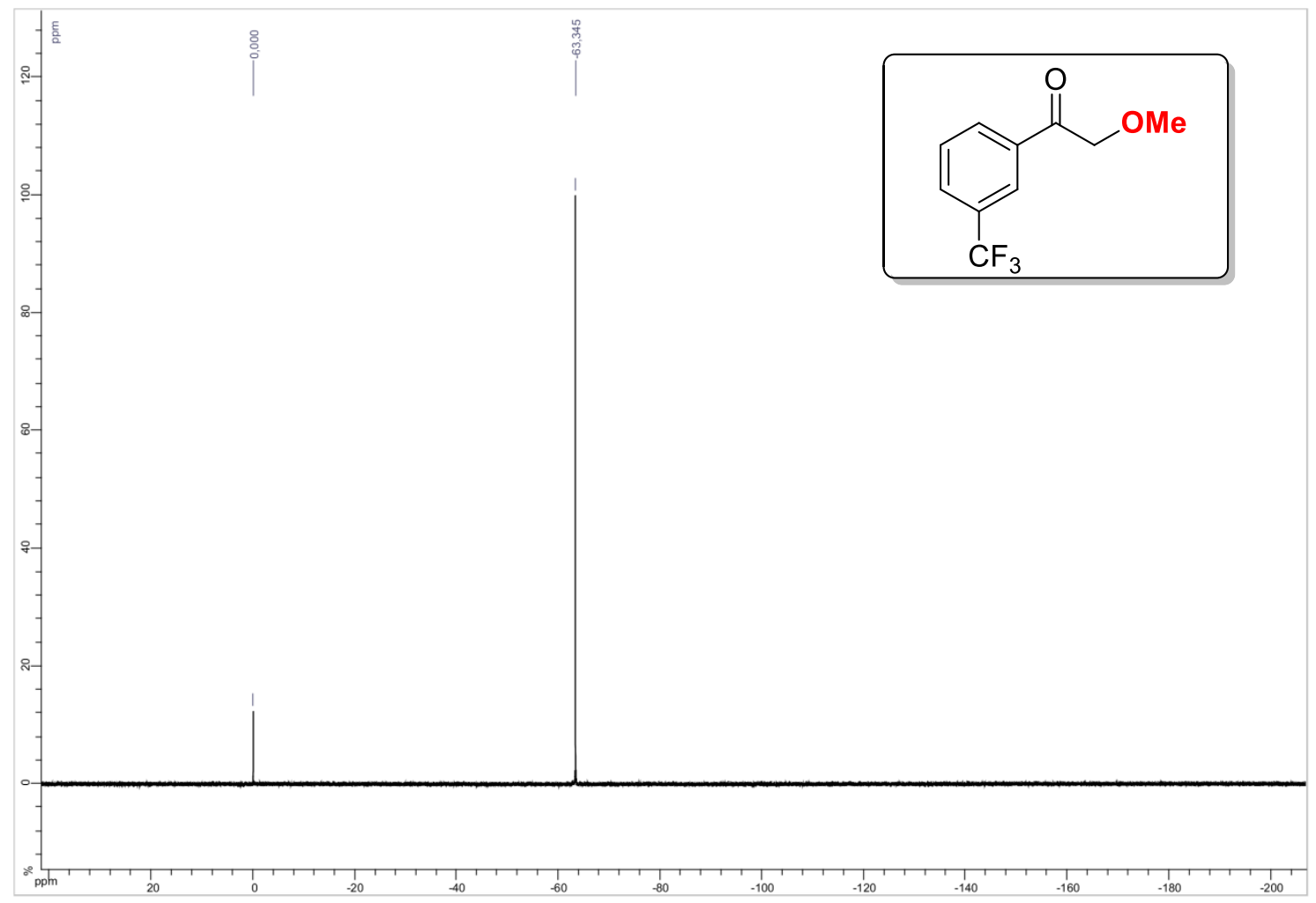


3-(2-Methoxyacetyl)benzonitrile 4j

${ }^{1}$ H NMR (300 MHz, CDCl 3 )

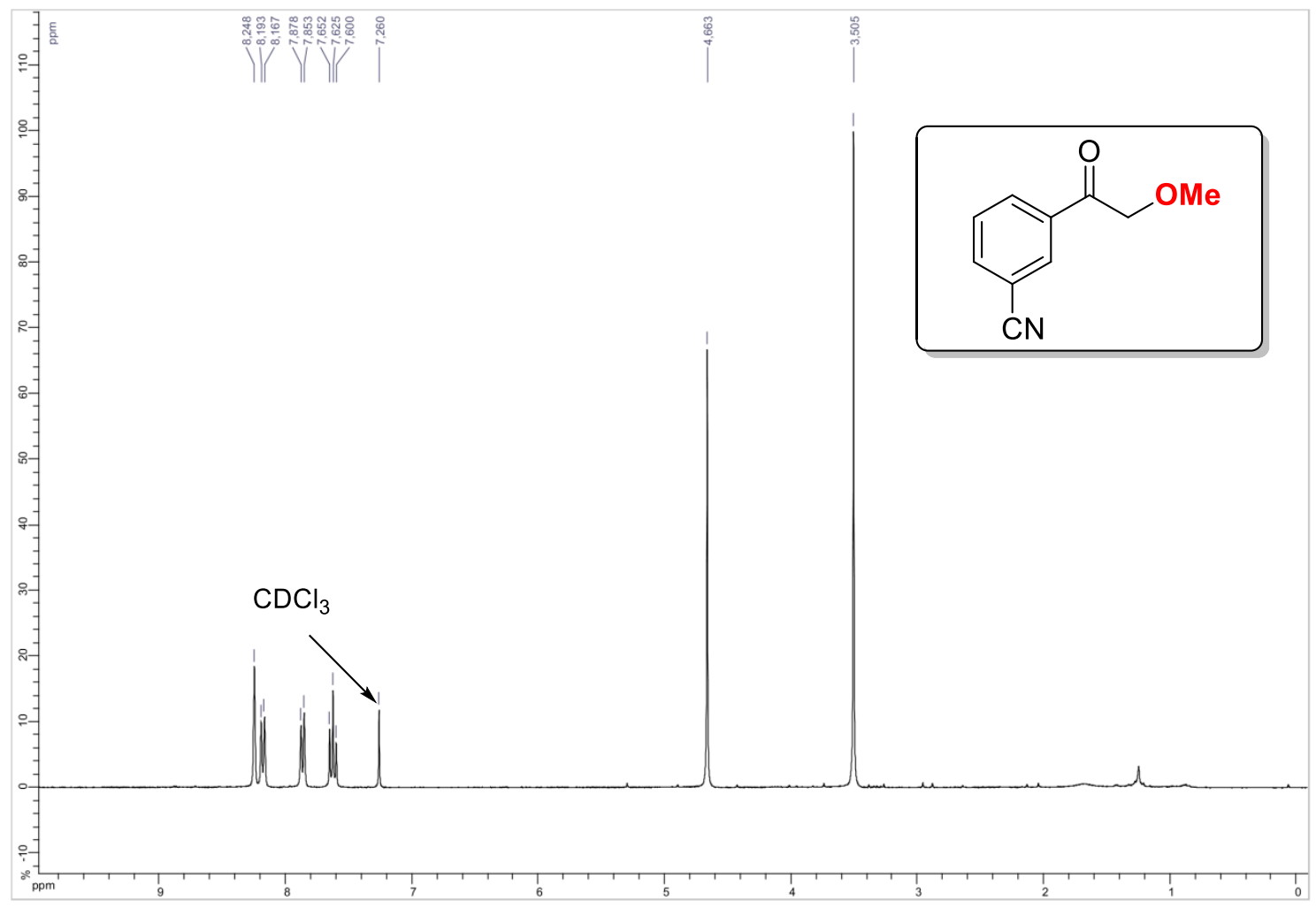

${ }^{13} \mathrm{C}$ NMR (75 MHz, CDCl3)

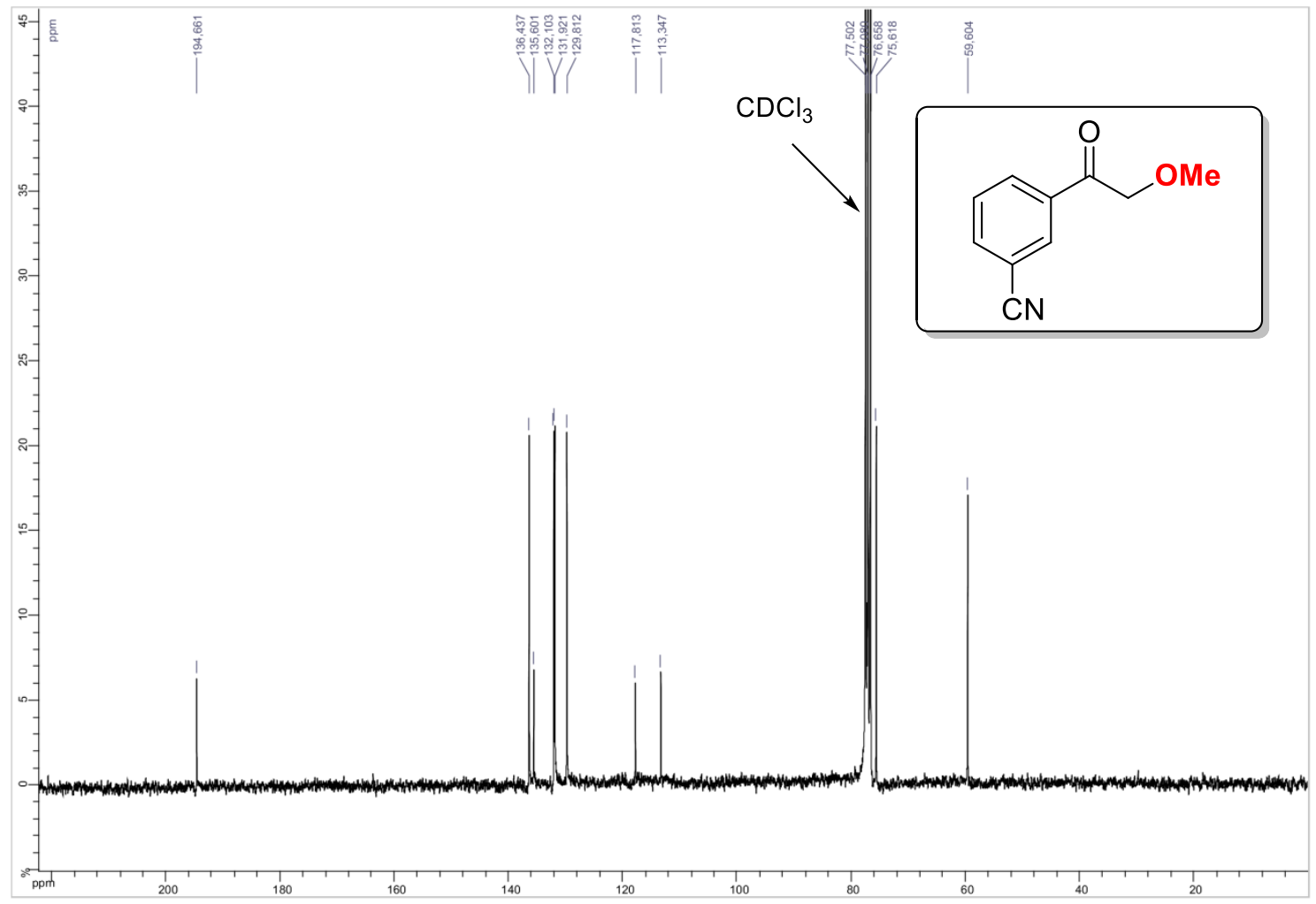


Tert-butyl 3-(2-methoxyacetyl)-1H-indole-1-carboxylate 4k

${ }^{1} \mathrm{H}$ NMR (300 MHz, $\left.\mathrm{CDCl}_{3}\right)$

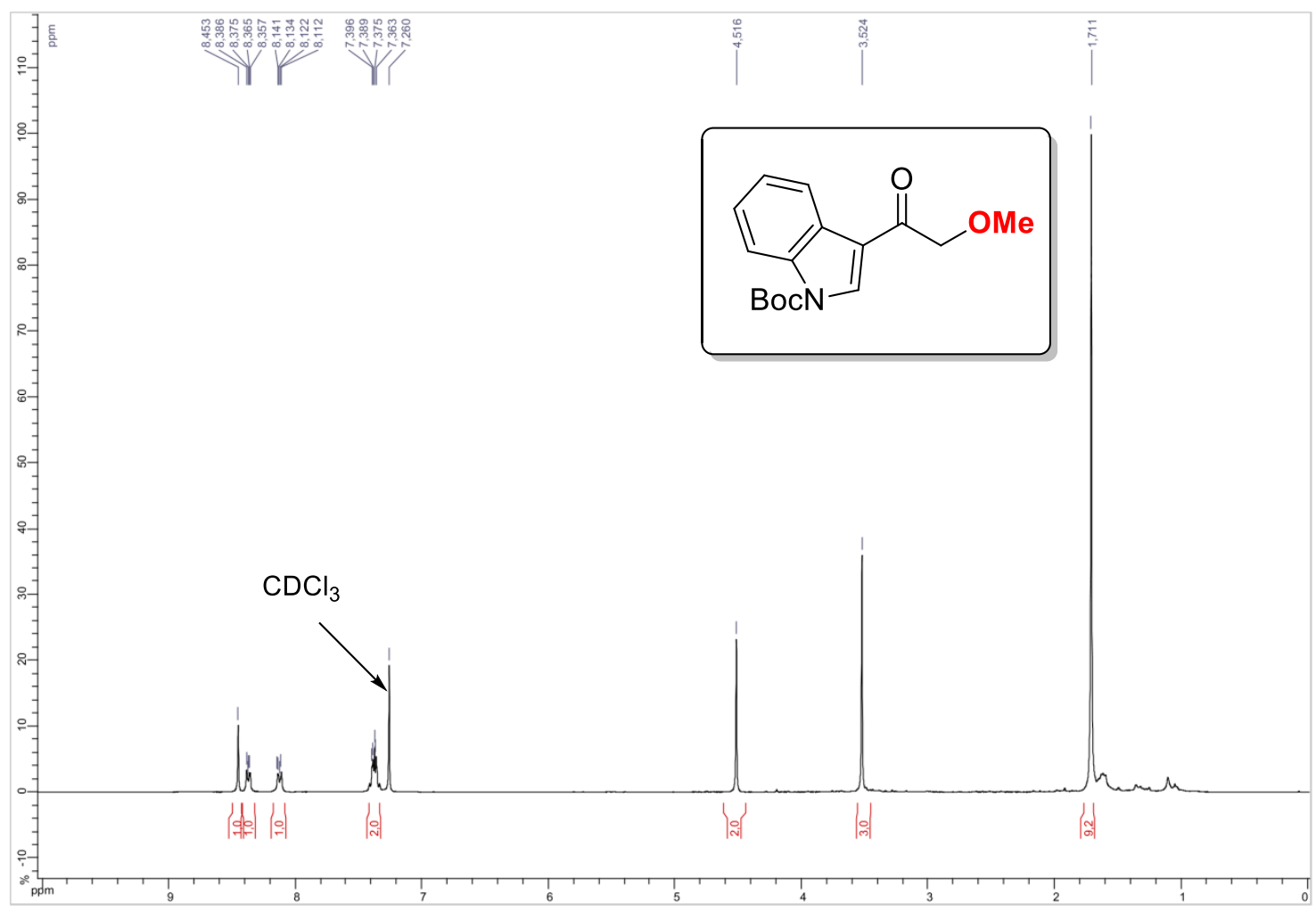

${ }^{13} \mathrm{C}$ NMR (75 $\left.\mathrm{MHz}, \mathrm{CDCl}_{3}\right)$

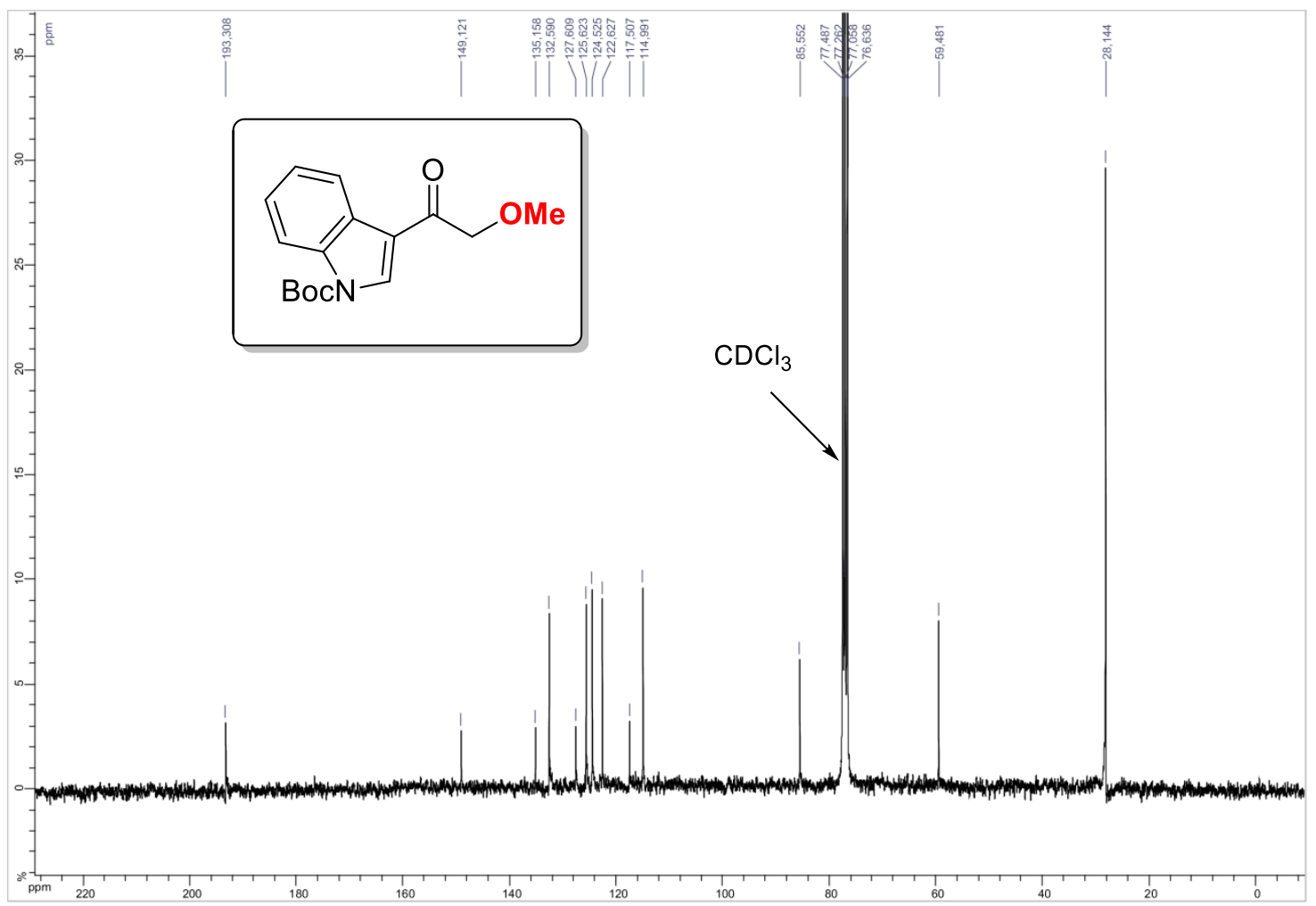


1-(Benzofuran-2-yl)-2-methoxyethanone 4l

${ }^{1} \mathrm{H}$ NMR (300 MHz, $\left.\mathrm{CDCl}_{3}\right)$

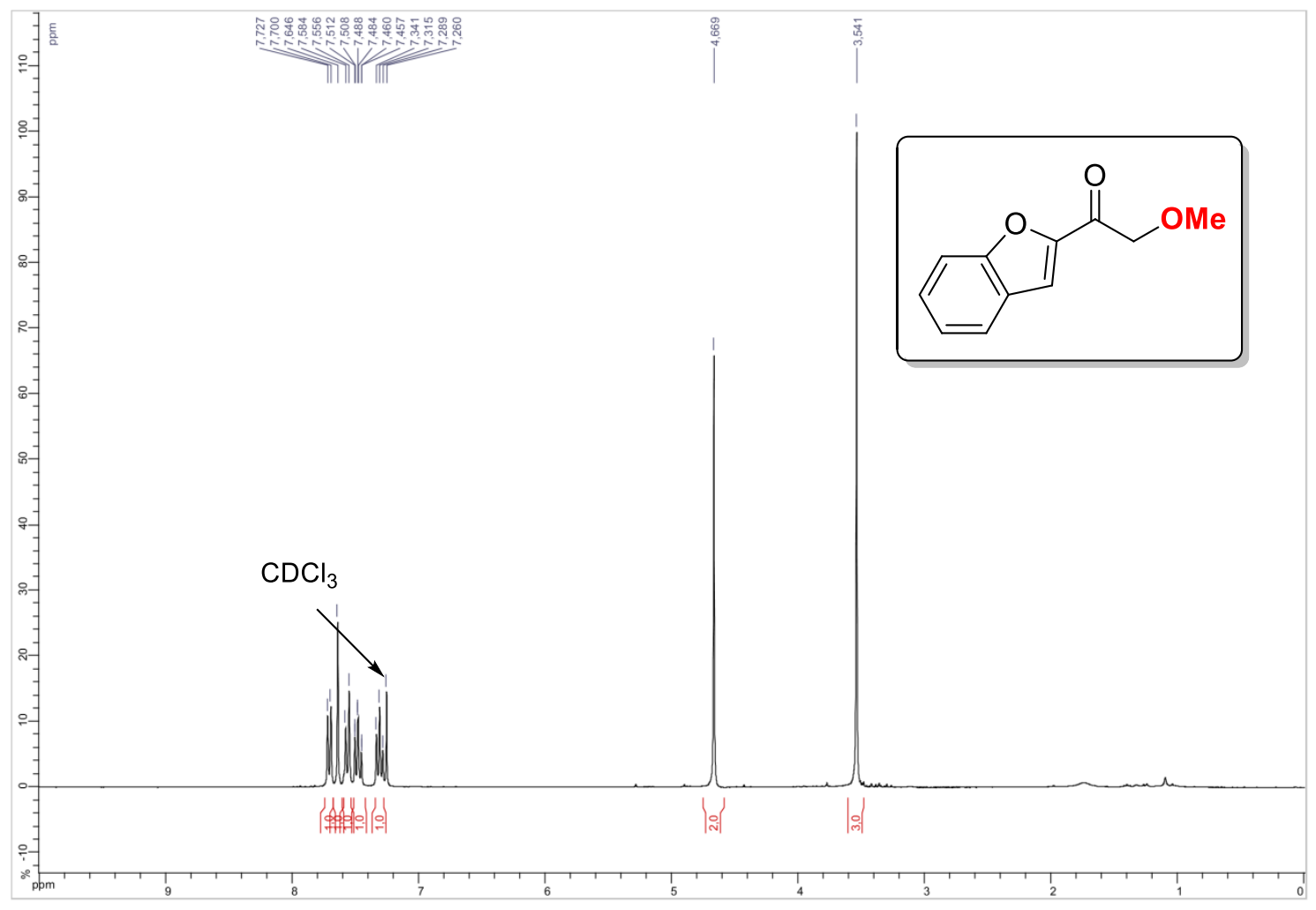

${ }^{13} \mathrm{C}$ NMR (75 MHz, CDCl3)

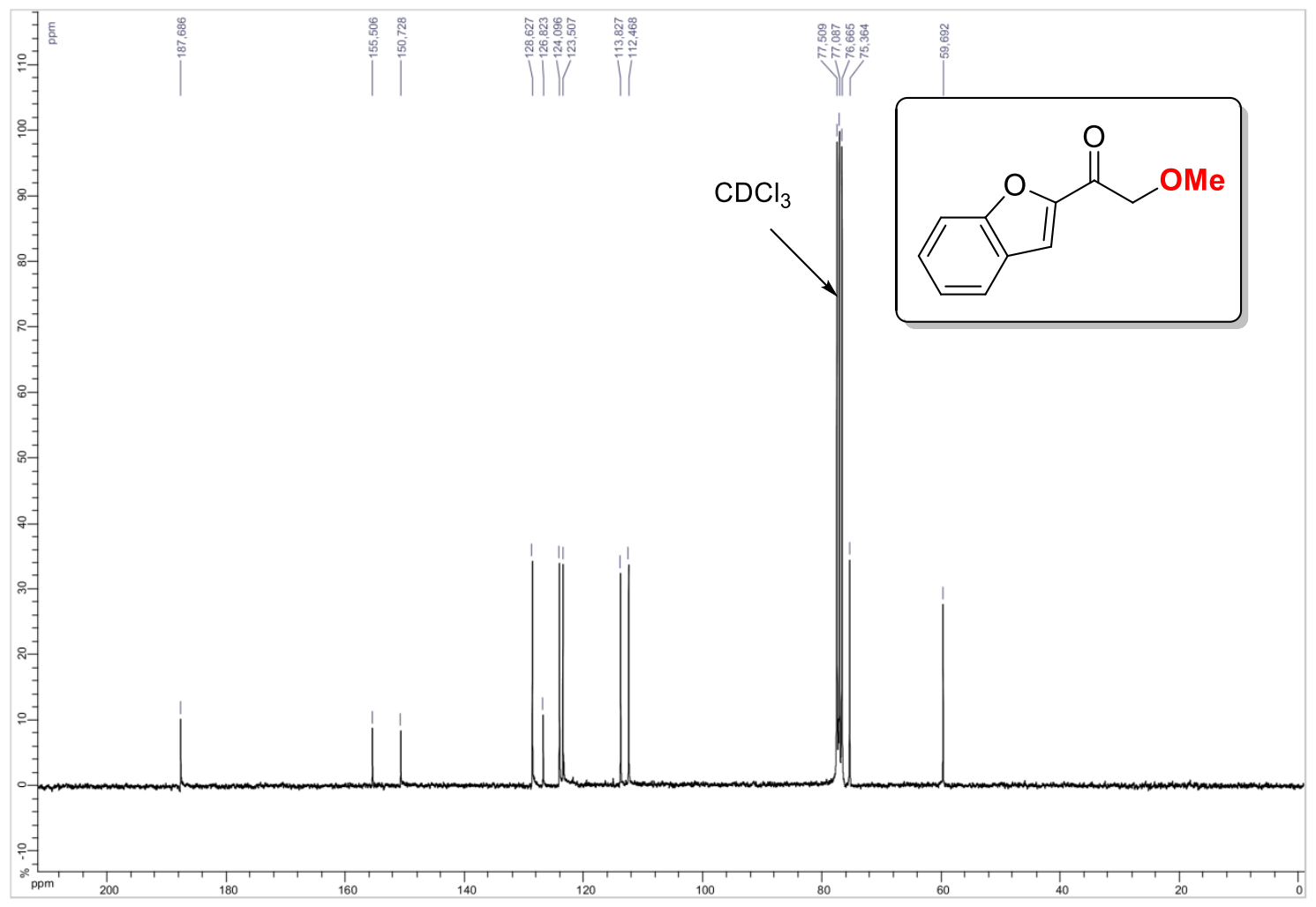


1-(Benzo[b]thiophen-2-yl)-2-methoxyethanone 4m

${ }^{1} \mathrm{H}$ NMR (300 MHz, $\left.\mathrm{CDCl}_{3}\right)$

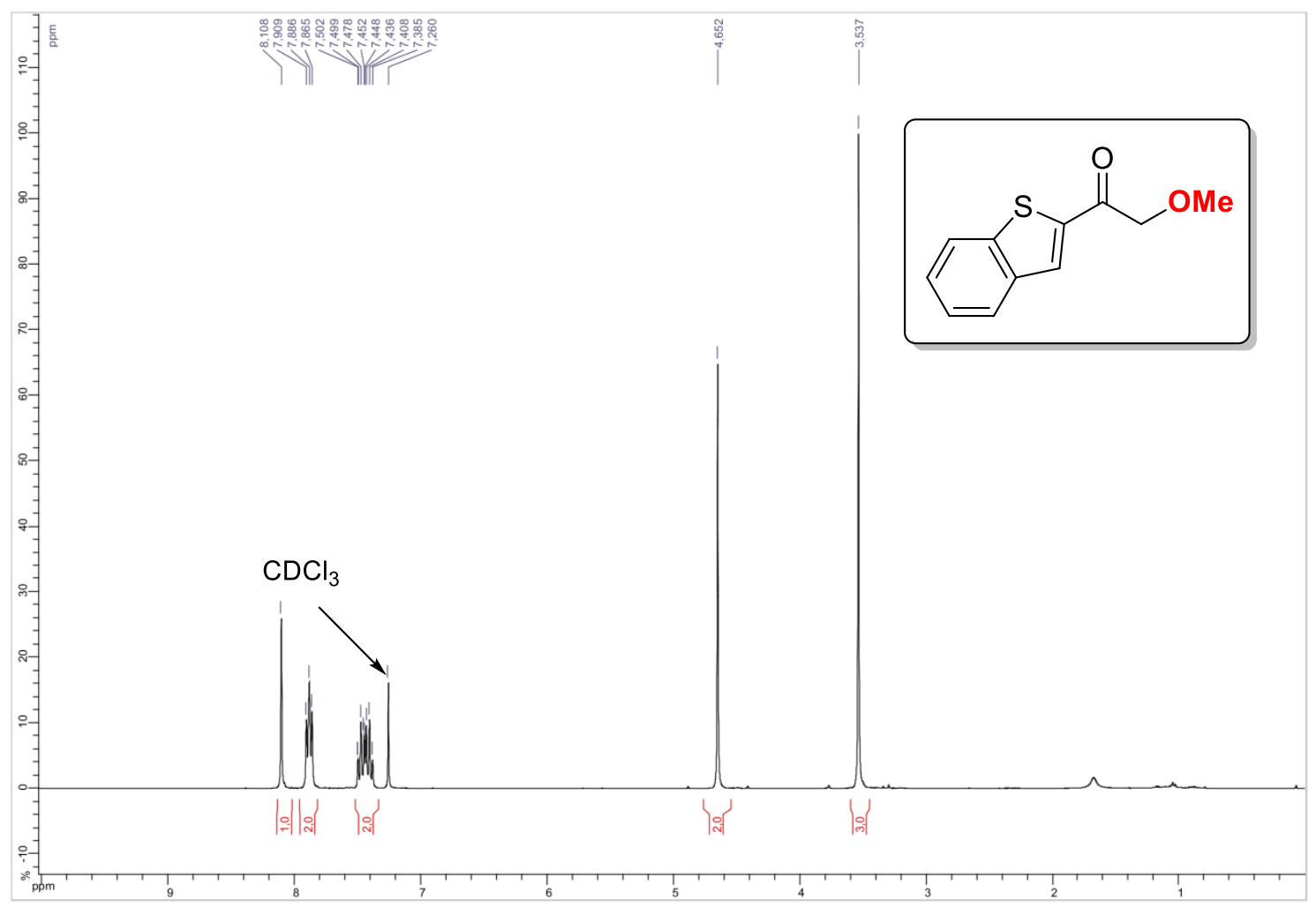

${ }^{13} \mathrm{C}$ NMR (75 MHz, CDCl3)

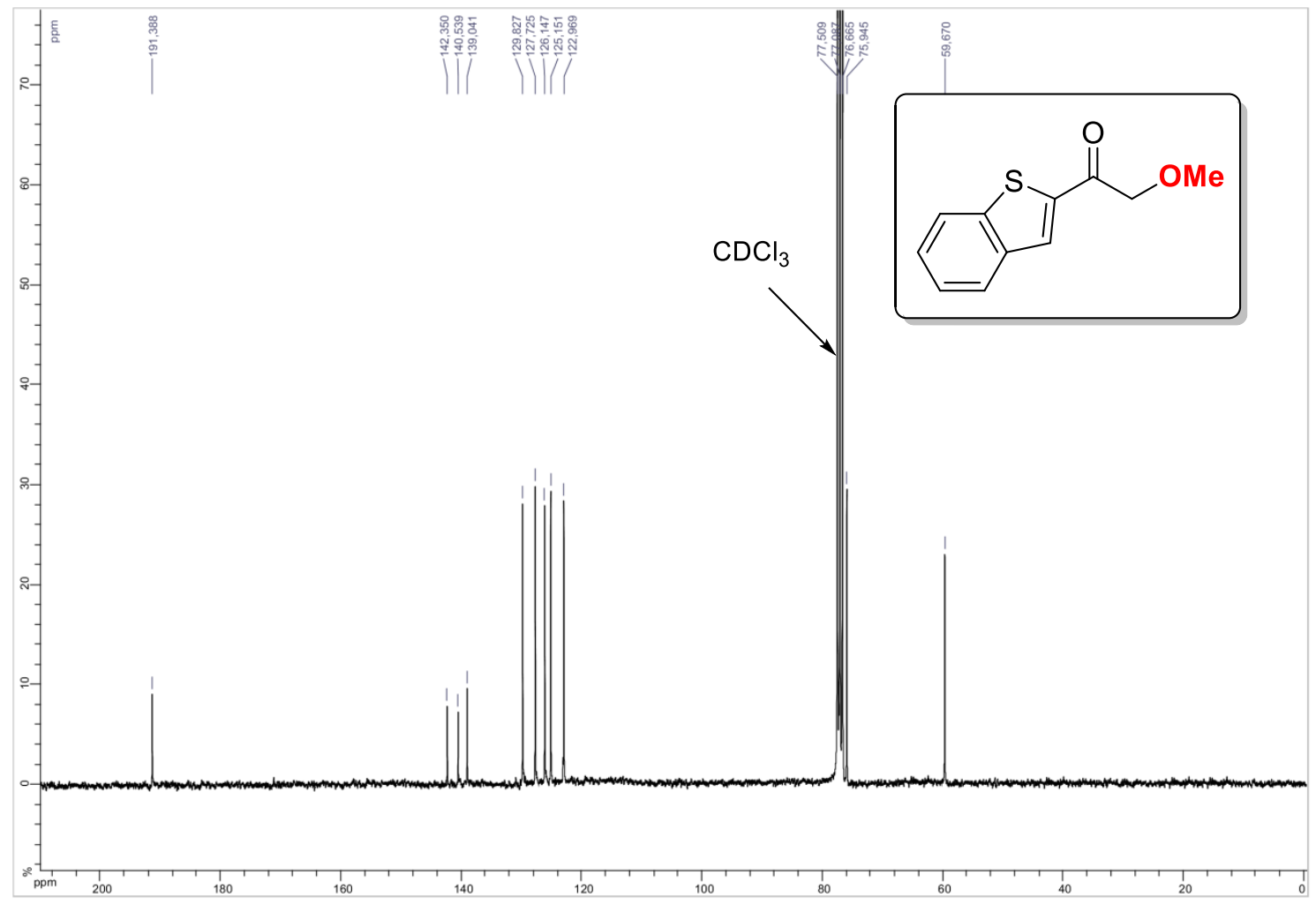


1-(4-Chlorophenyl)-2-methoxypropan-1-one 4n

${ }^{1}$ H NMR (300 MHz, CDCl 3 )

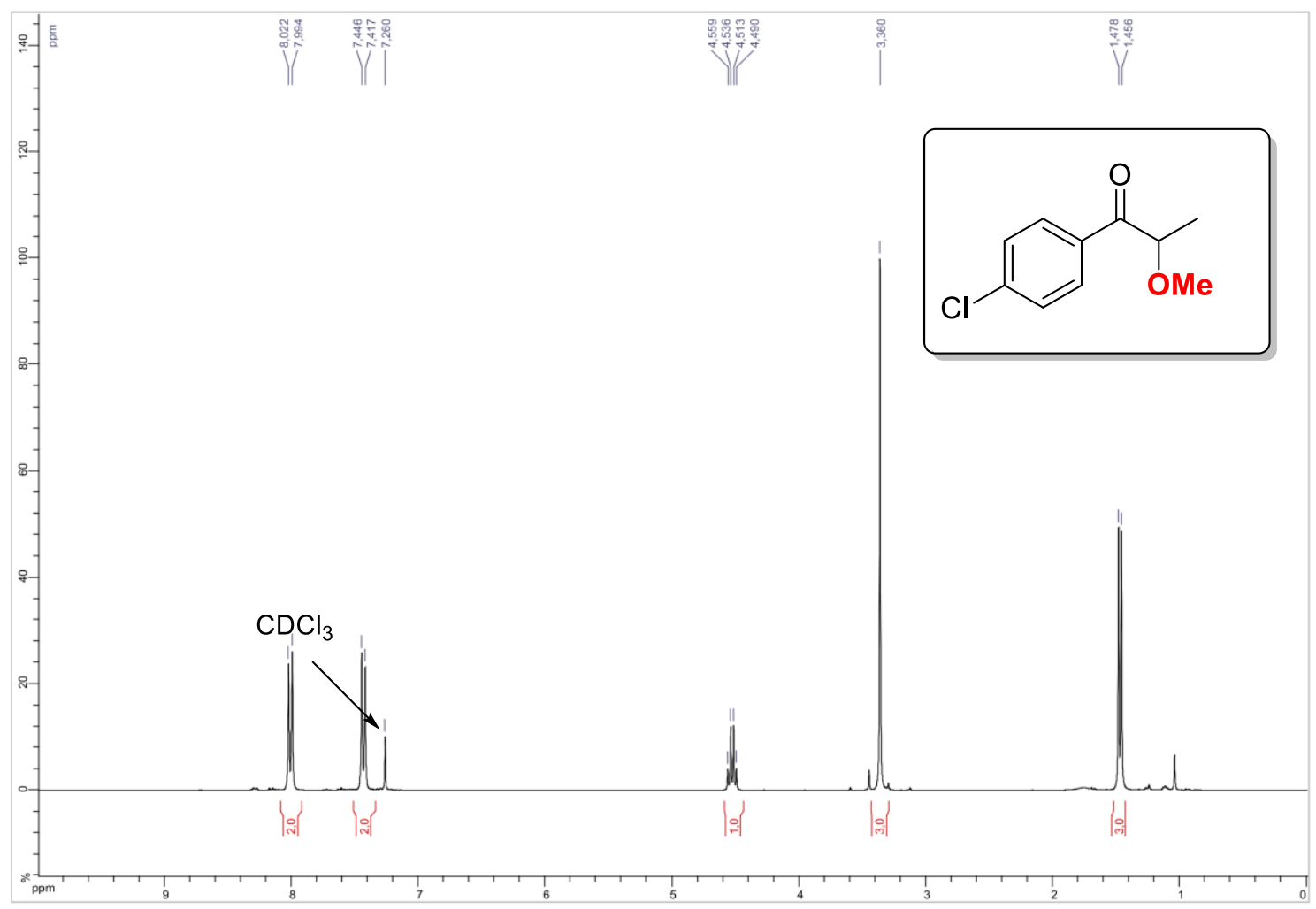

${ }^{13} \mathrm{C}$ NMR (75 MHz, CDCl3)

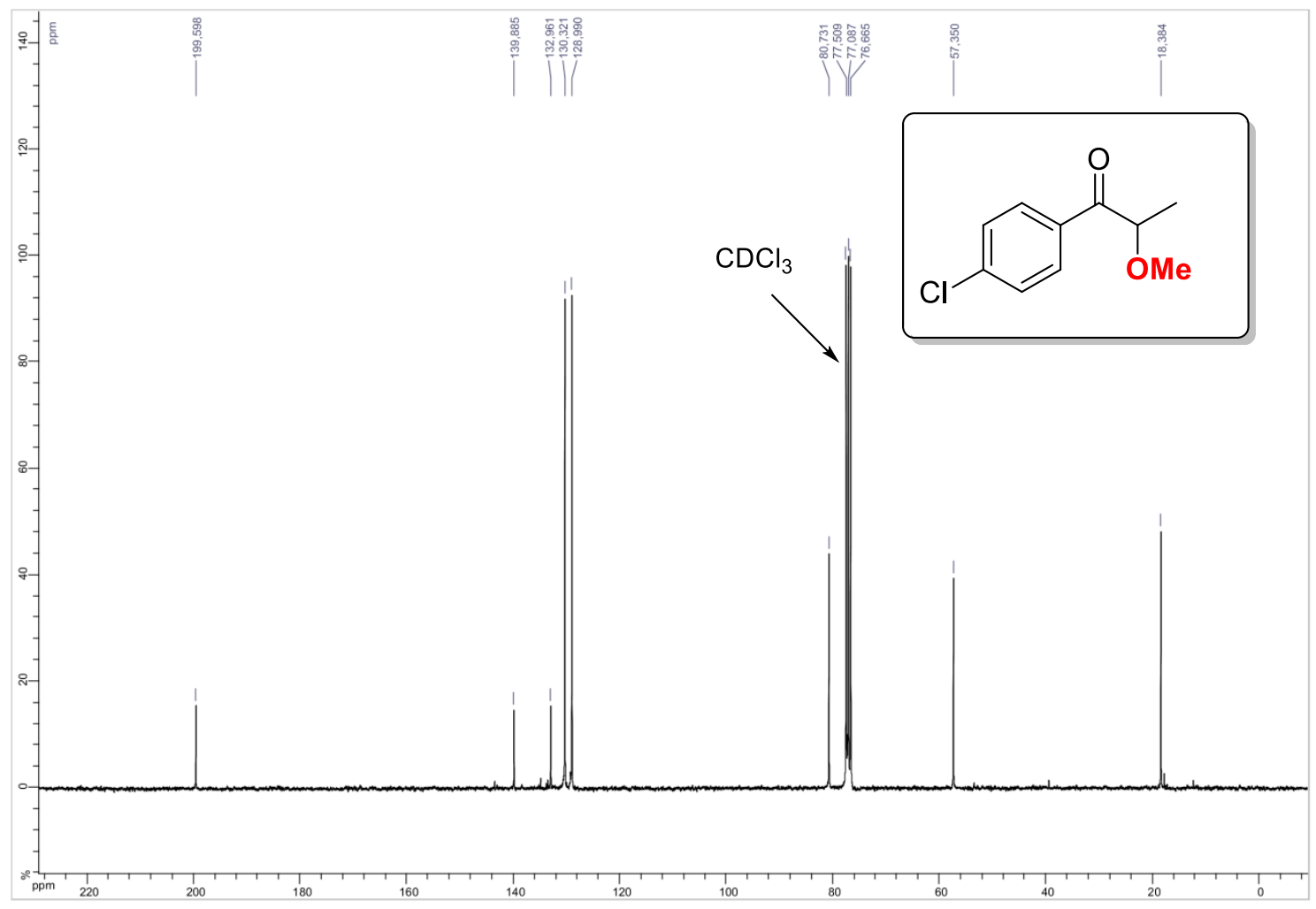


2-Methoxy-2-methyl-1-phenylpropan-1-one 4o

${ }^{1} \mathrm{H}$ NMR (300 MHz, $\left.\mathrm{CDCl}_{3}\right)$

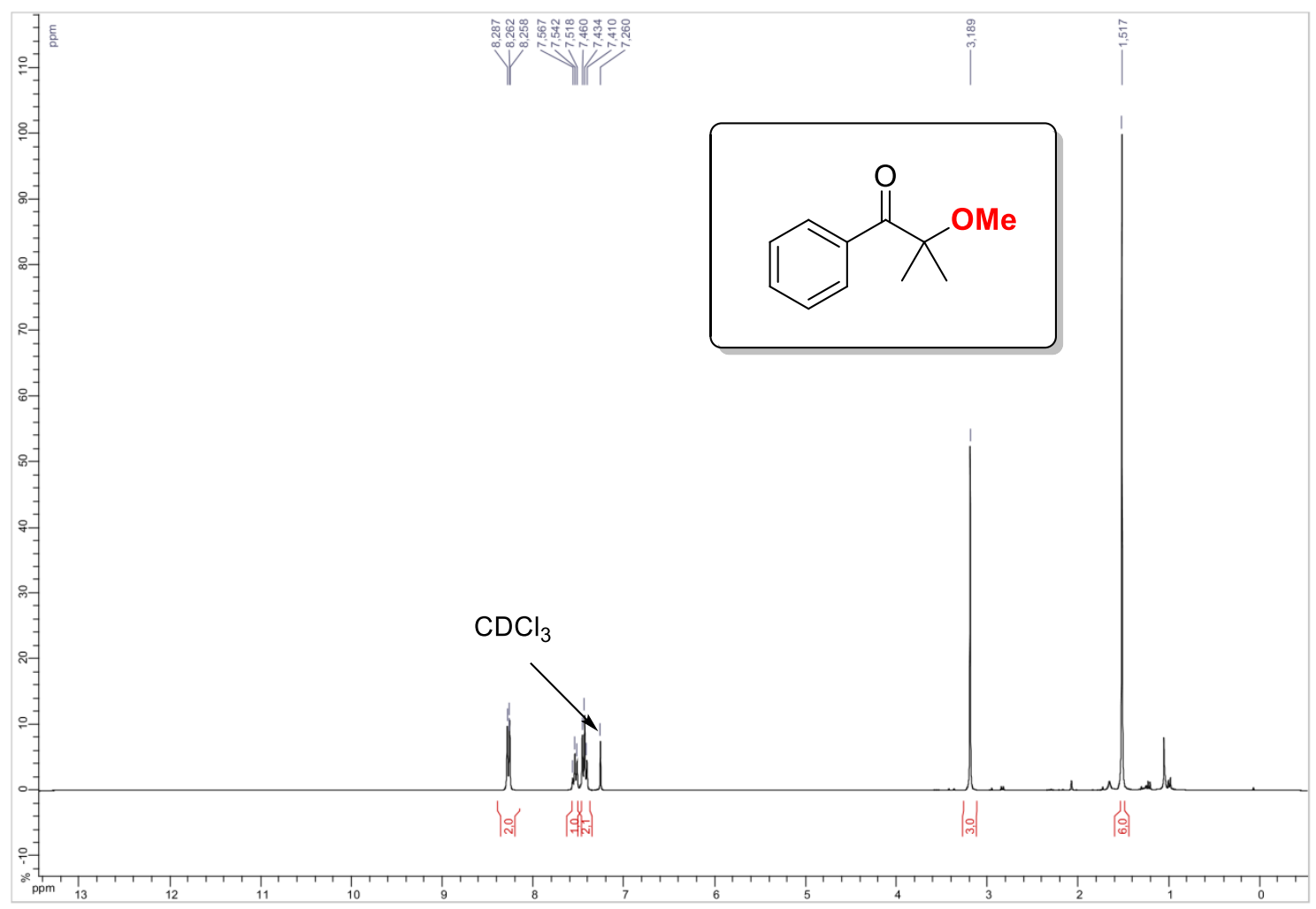

${ }^{13} \mathrm{C}$ NMR (75 MHz, CDCl3)

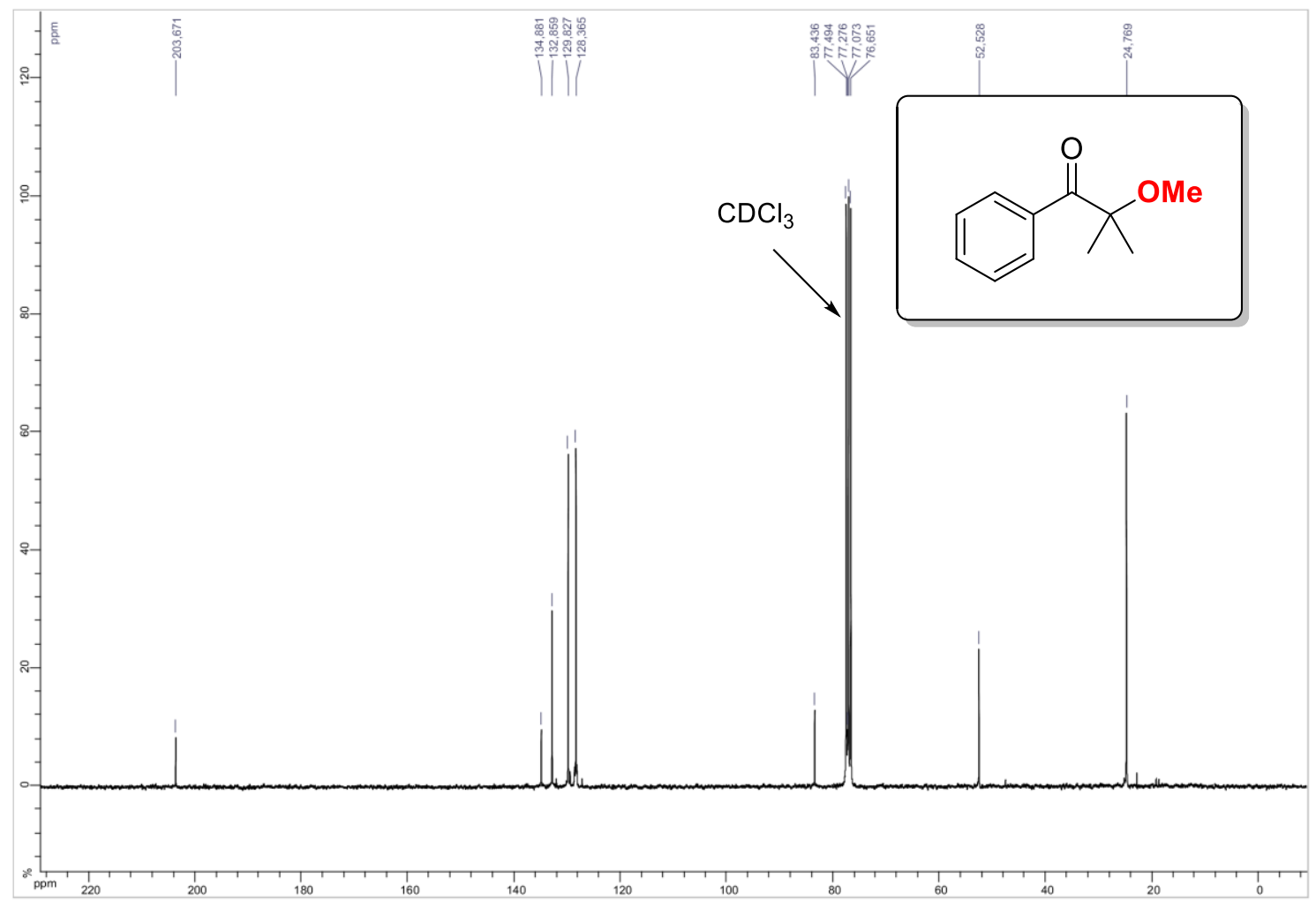


2-Methoxy-3,4-dihydronaphthalen-1(2H)-one 4p

${ }^{1} \mathrm{H}$ NMR (300 MHz, $\left.\mathrm{CDCl}_{3}\right)$

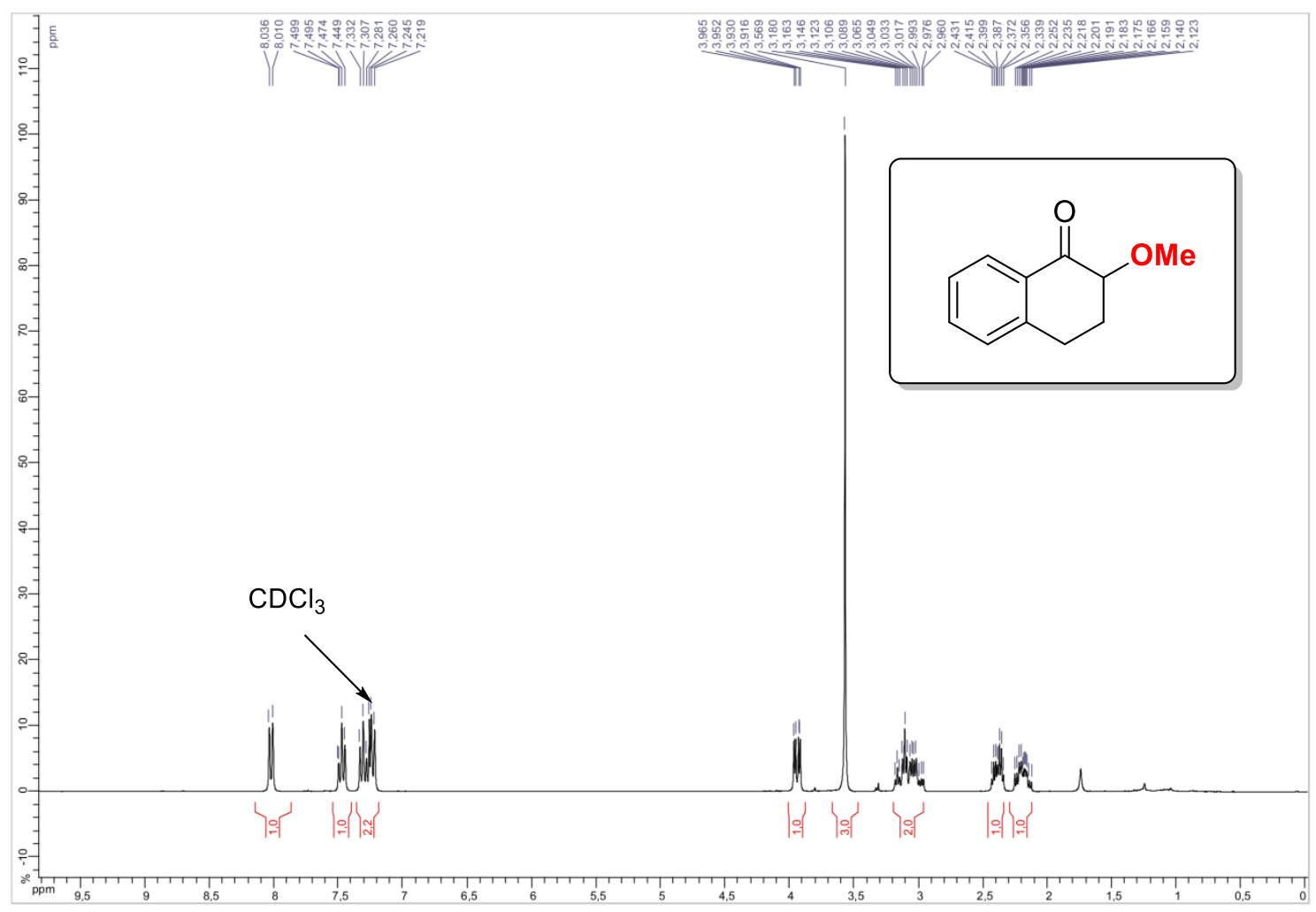

${ }^{13} \mathrm{C}$ NMR (75 MHz, CDCl3)

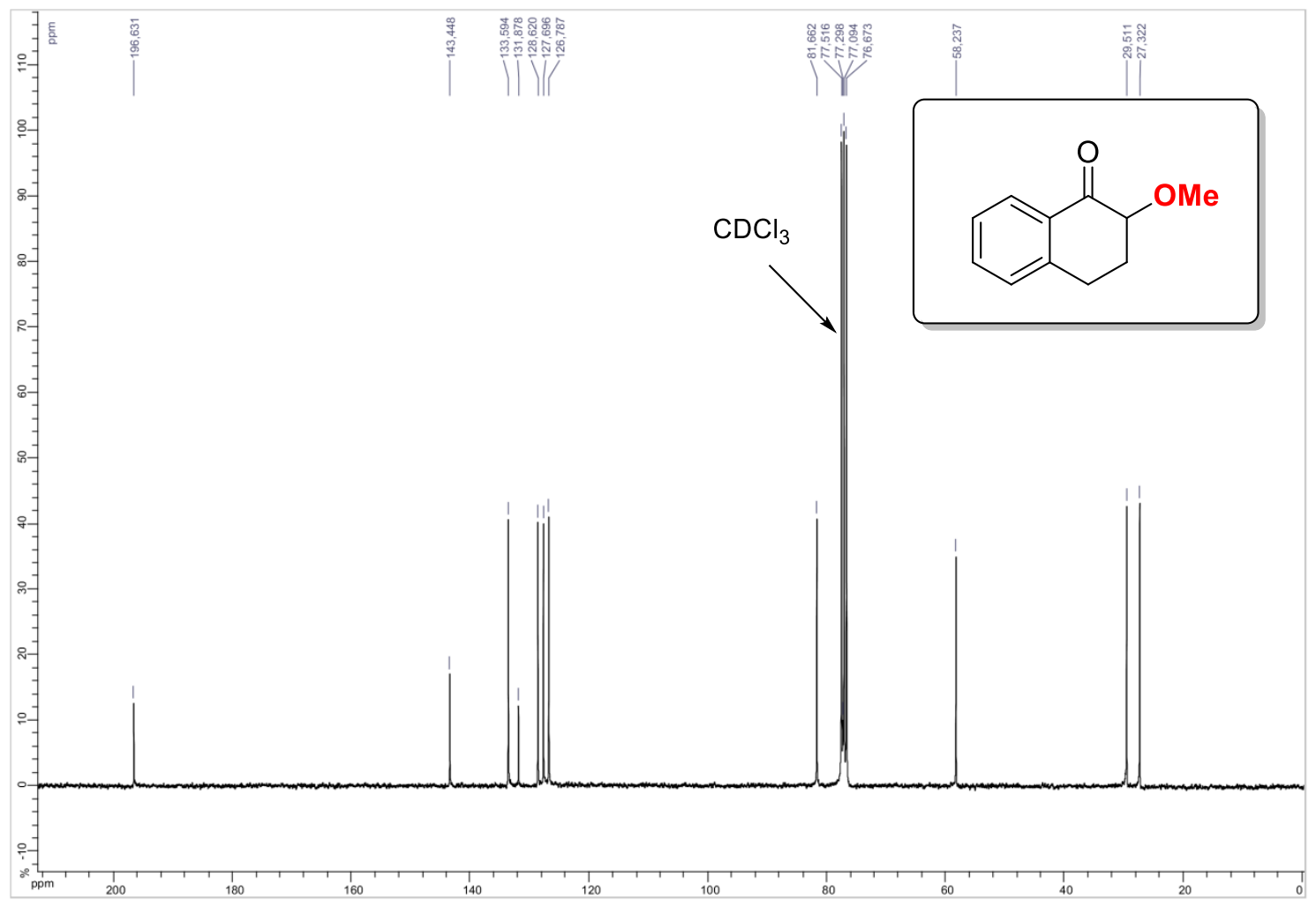


3-Methoxychroman-4-one 4q

${ }^{1} \mathrm{H}$ NMR (300 MHz, $\left.\mathrm{CDCl}_{3}\right)$

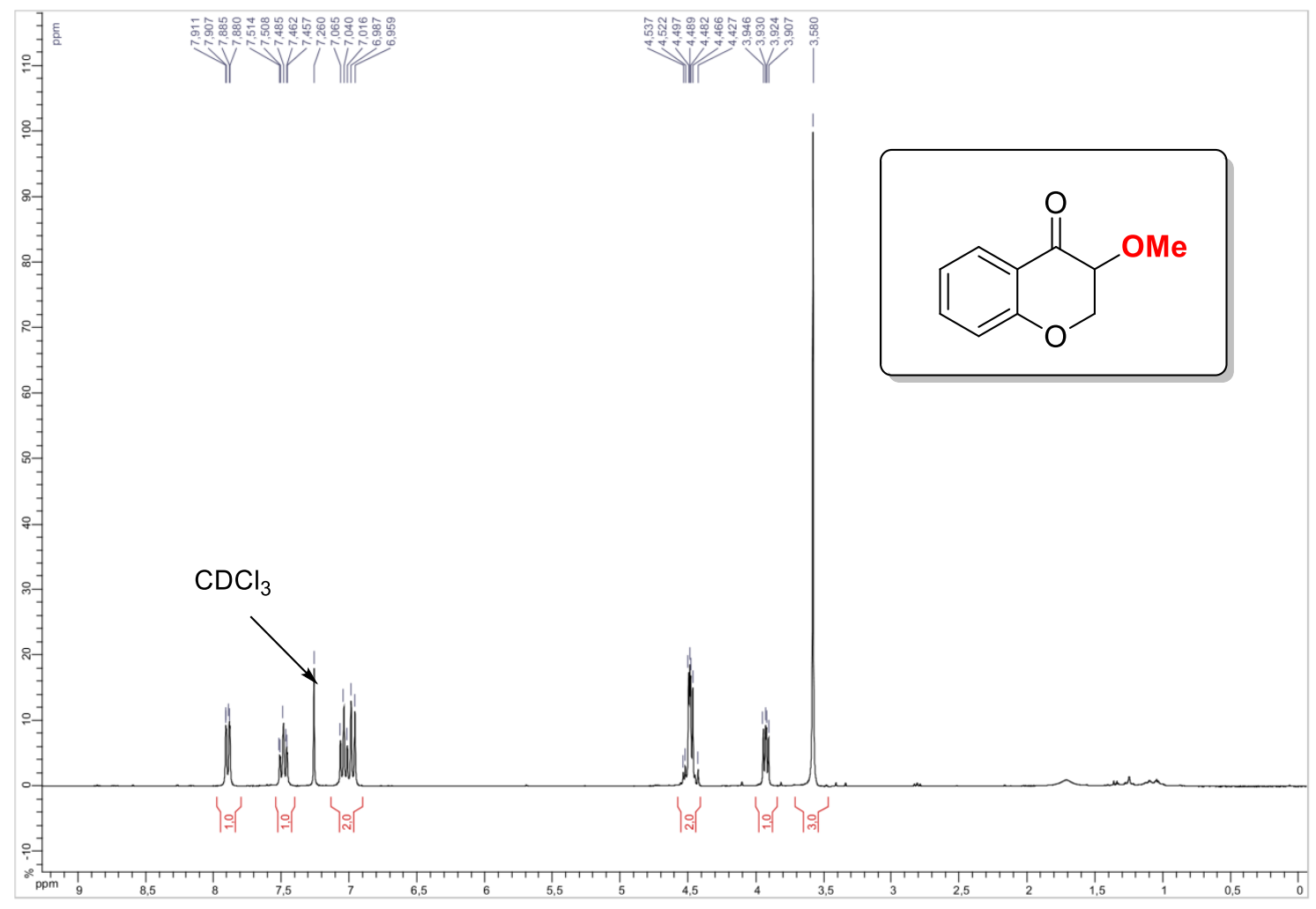

${ }^{13} \mathrm{C}$ NMR (75 MHz, CDCl3)

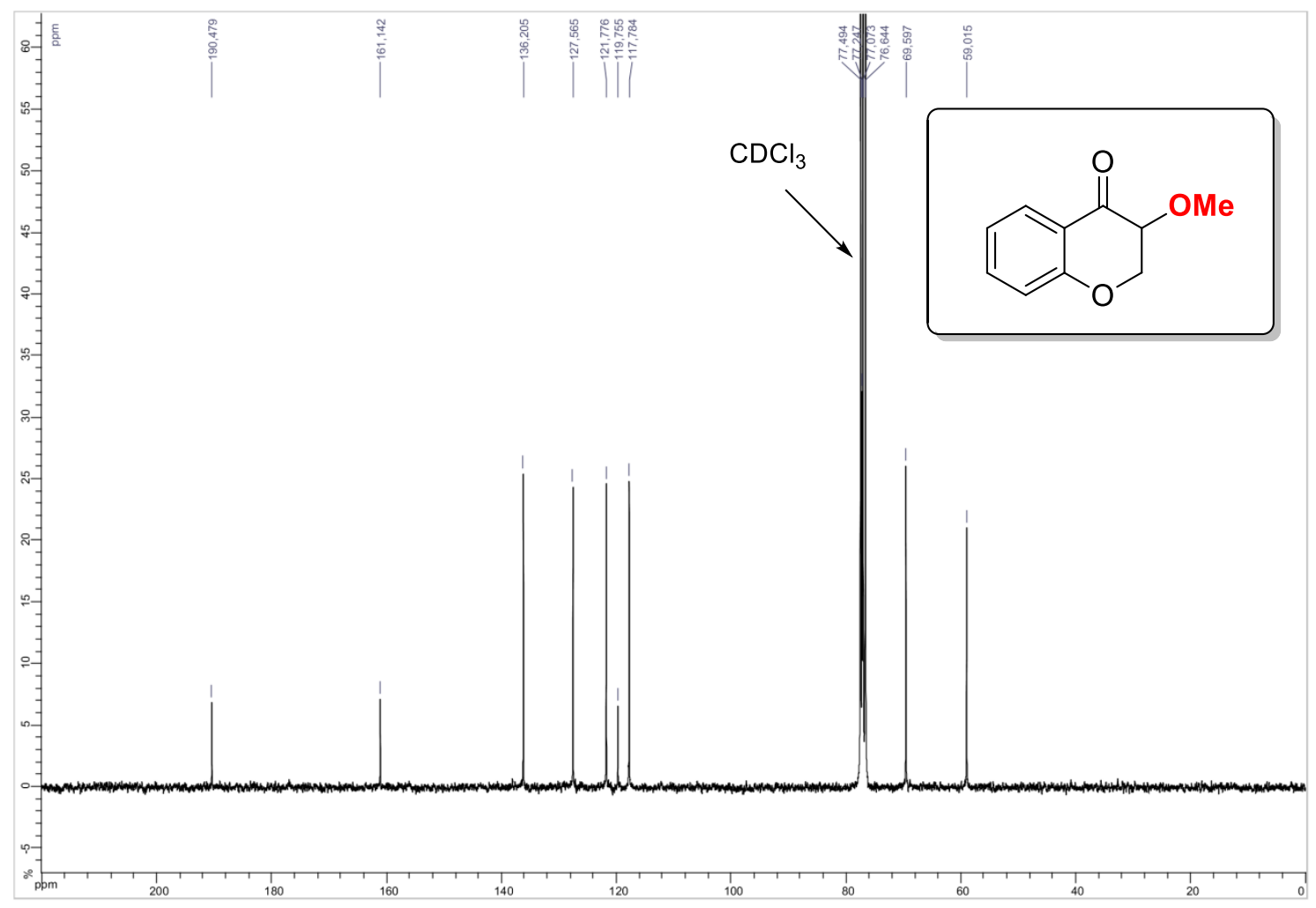


6-Methoxy-6,7,8,9-tetrahydro-5H-benzo[7]annulen-5-one 4r

${ }^{1} \mathrm{H}$ NMR (300 MHz, $\left.\mathrm{CDCl}_{3}\right)$

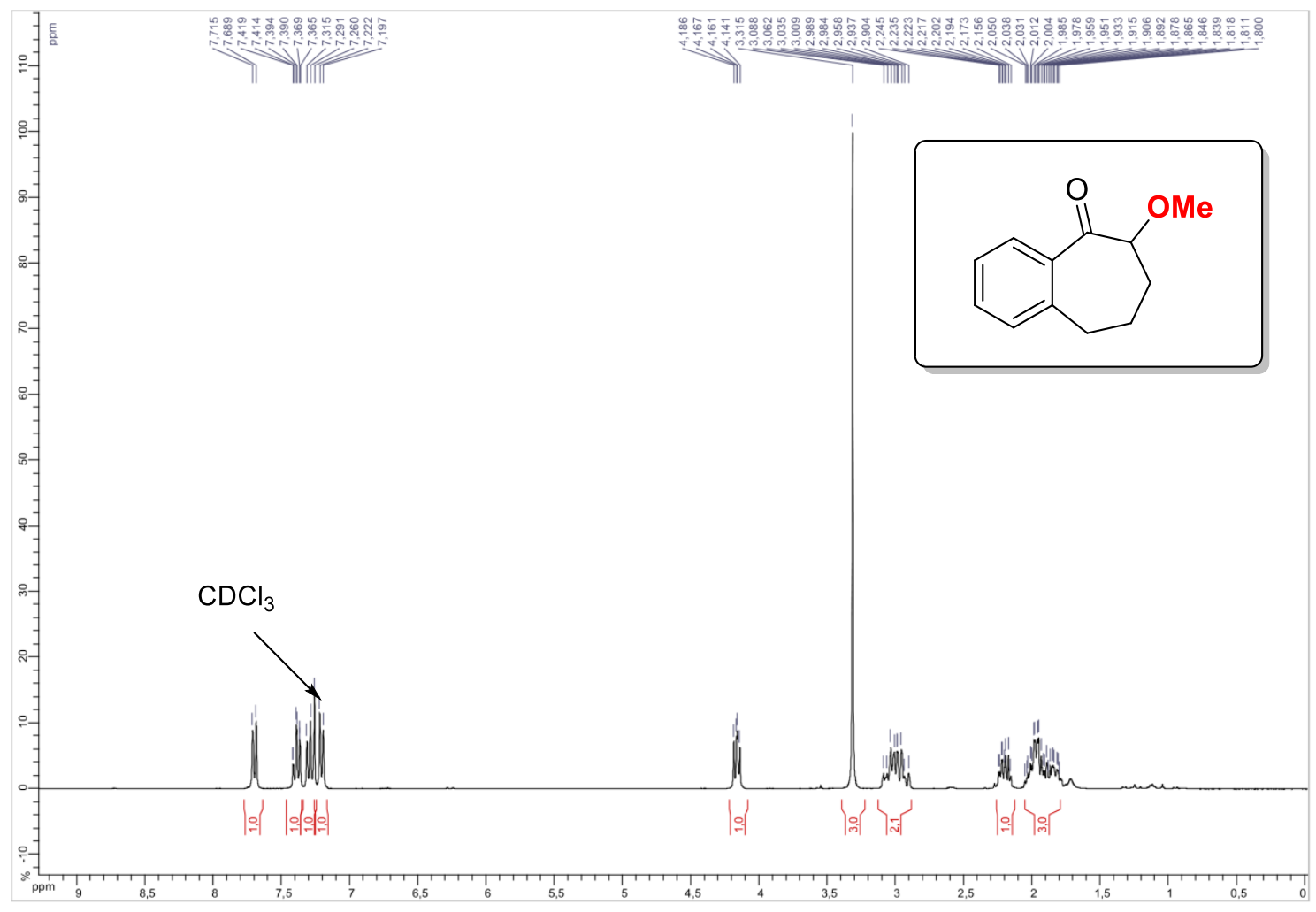

${ }^{13} \mathrm{C}$ NMR (75 MHz, CDCl3)

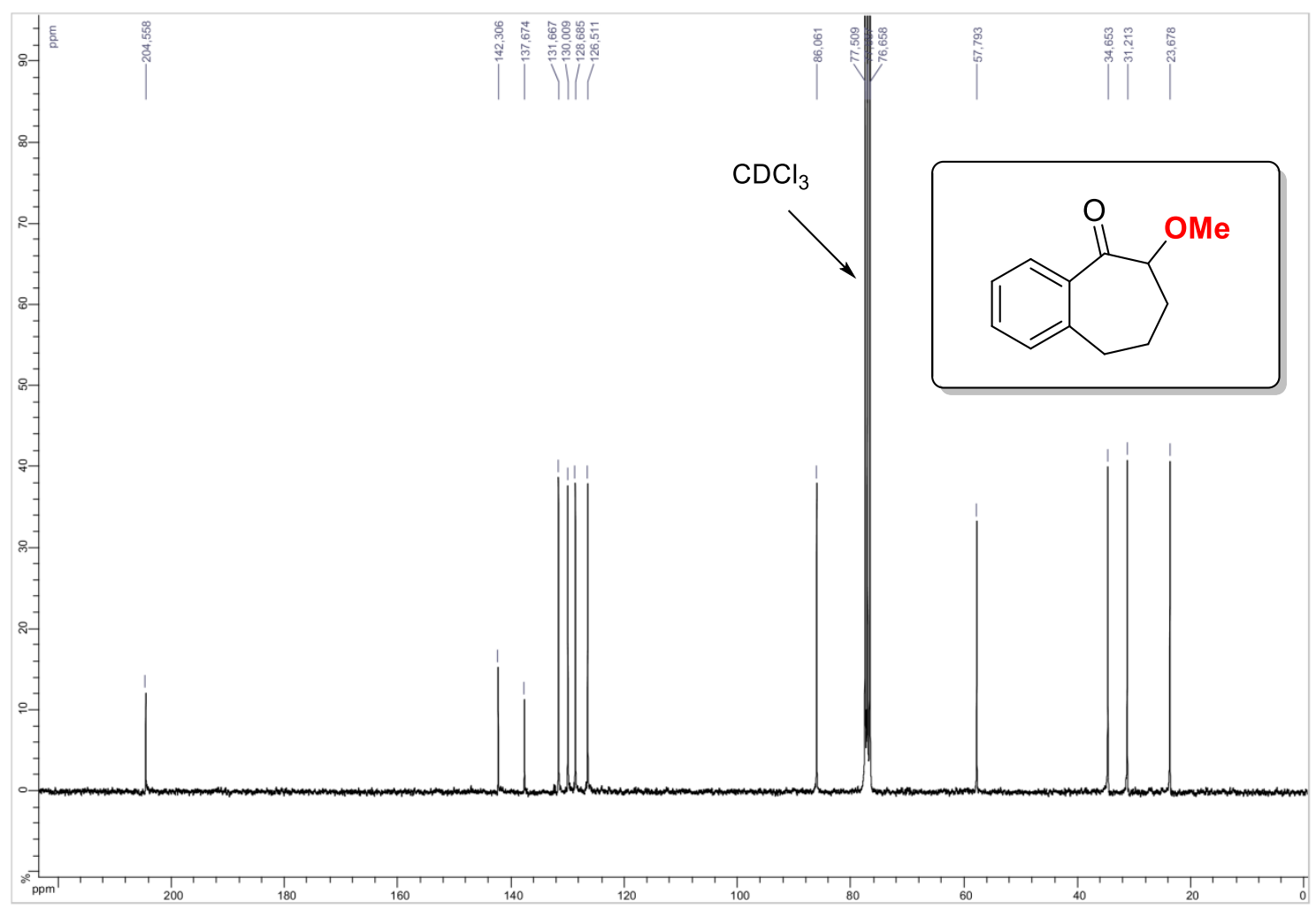


2-Methoxy-2,3-dihydro-1H-inden-1-one 4s

${ }^{1} \mathrm{H}$ NMR (300 MHz, $\left.\mathrm{CDCl}_{3}\right)$

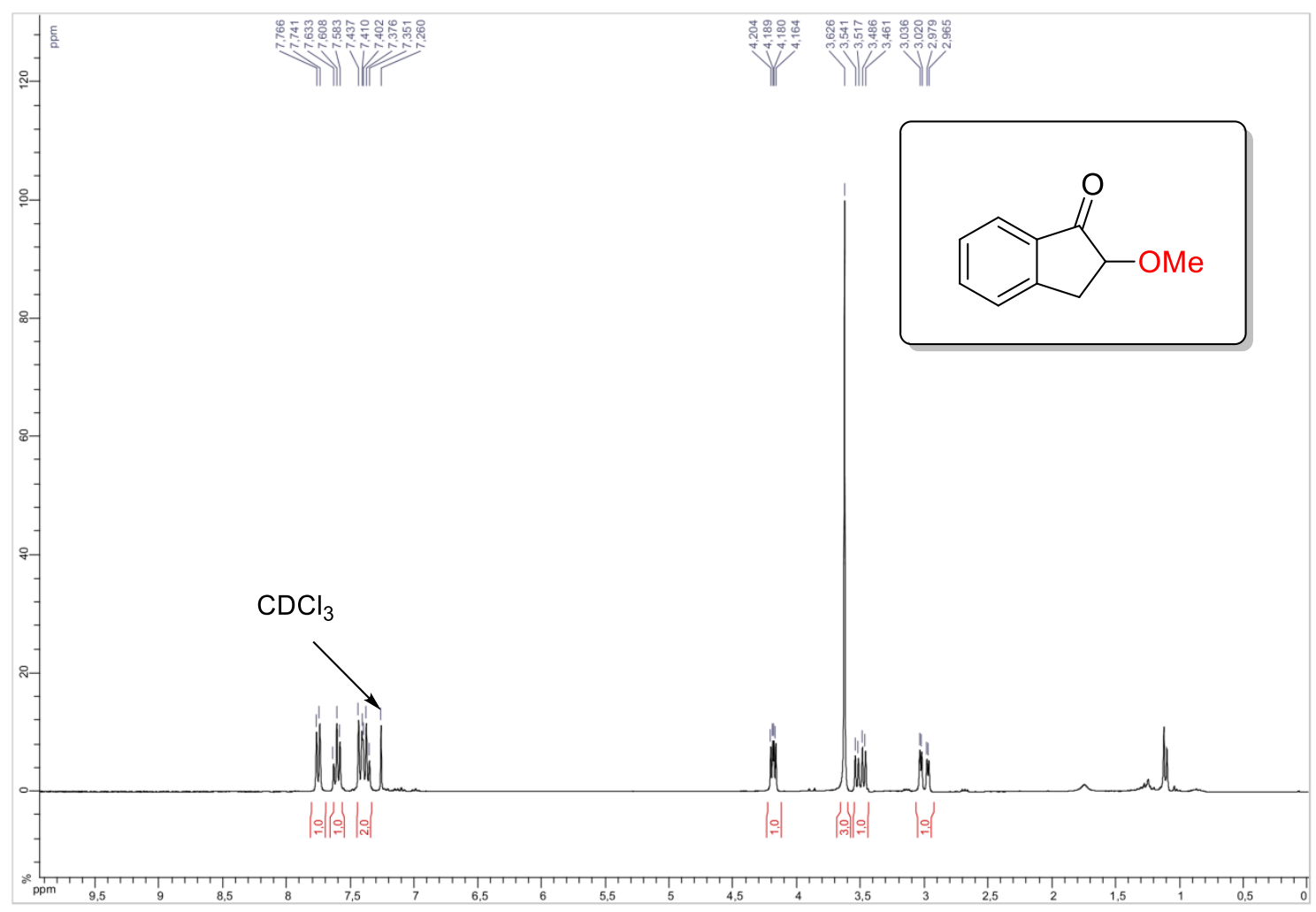

${ }^{13} \mathrm{C}$ NMR (75 MHz, CDCl3)

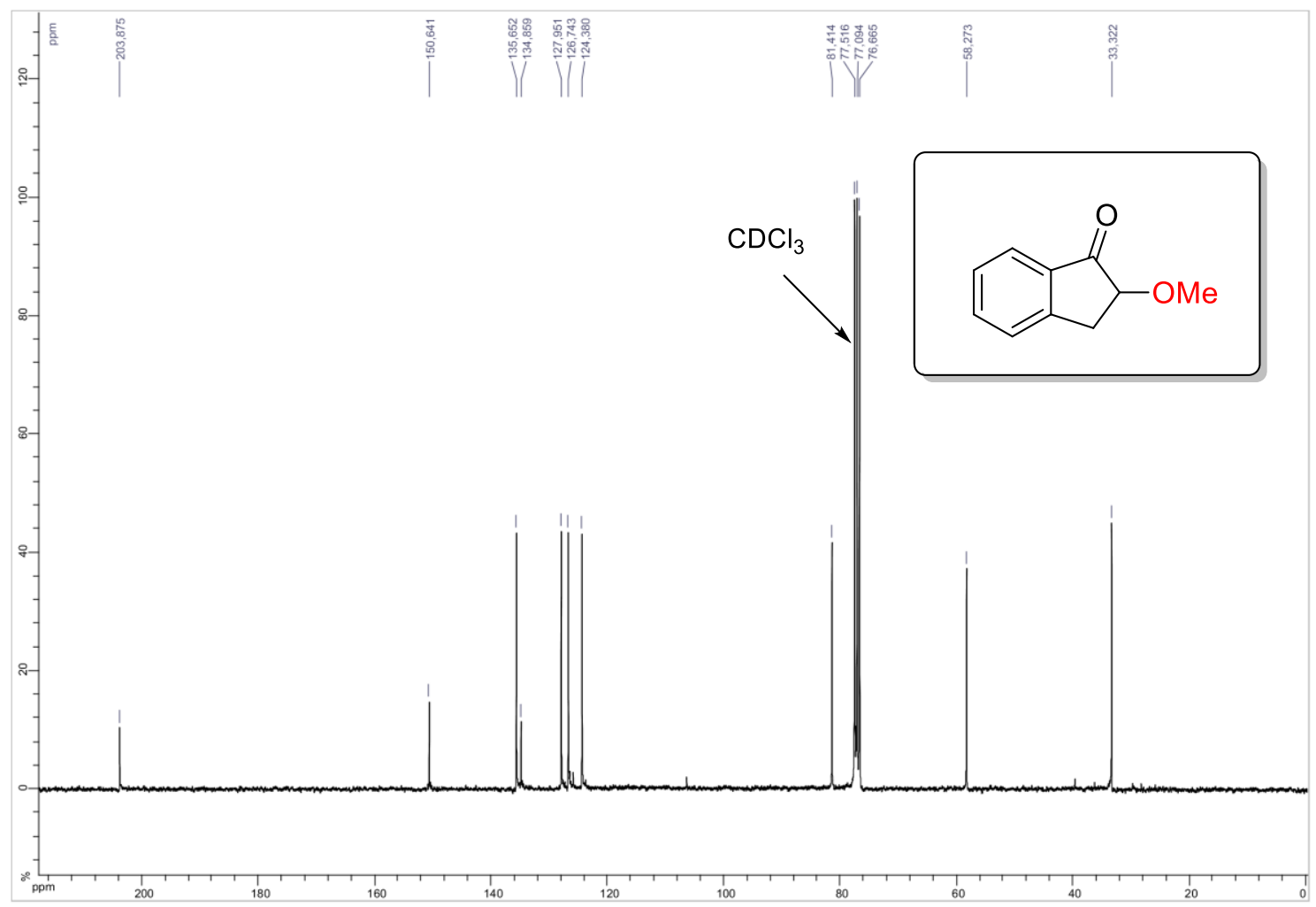


Tert-butyl 2-methoxy-1-oxo-2,3-dihydro-1H-indene-2-carboxylate $4 \mathrm{t}$ ${ }^{1} \mathrm{H}$ NMR (300 MHz, CDCl 3$)$

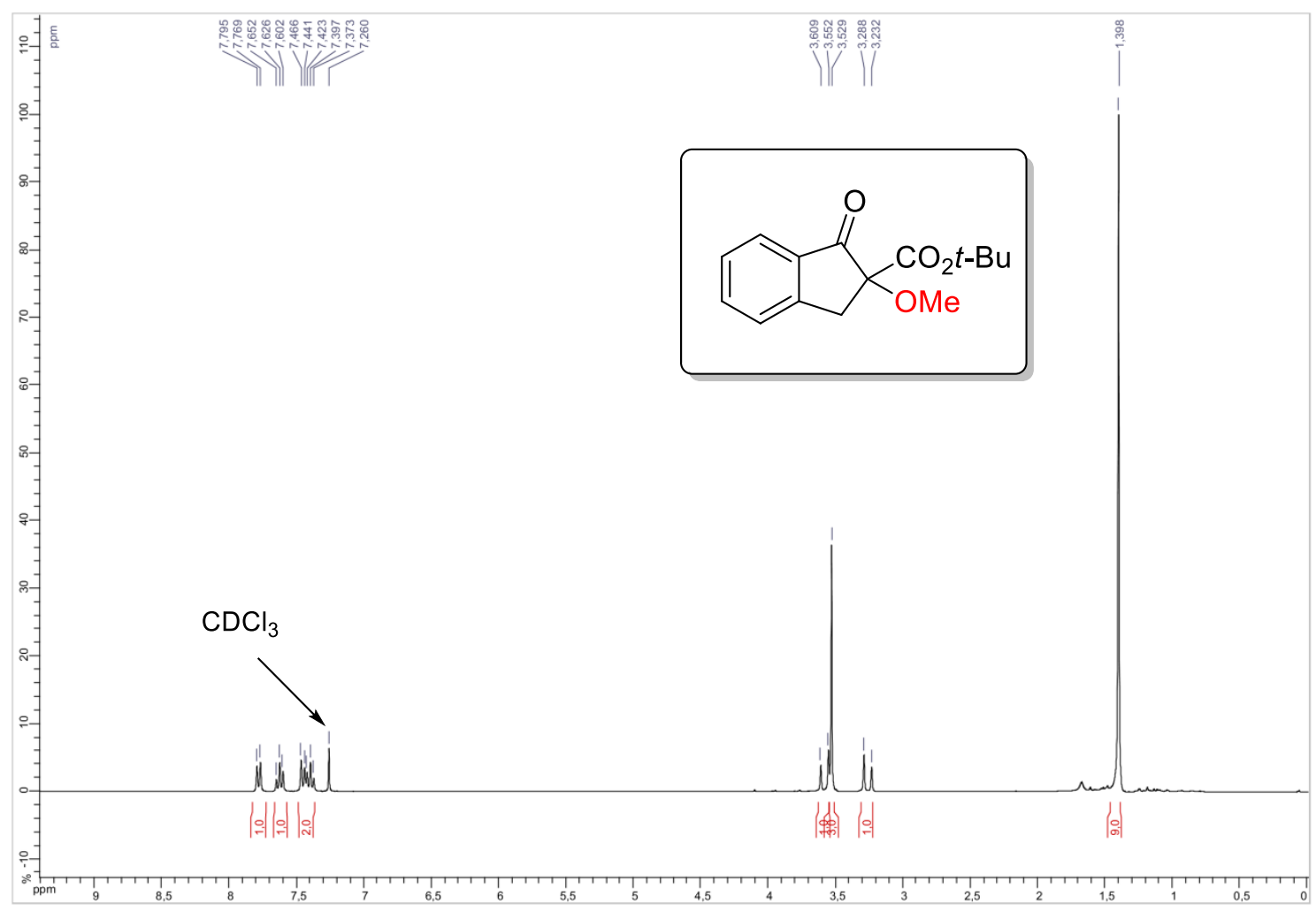

${ }^{13} \mathrm{C}$ NMR (75 MHz, CDCl3)

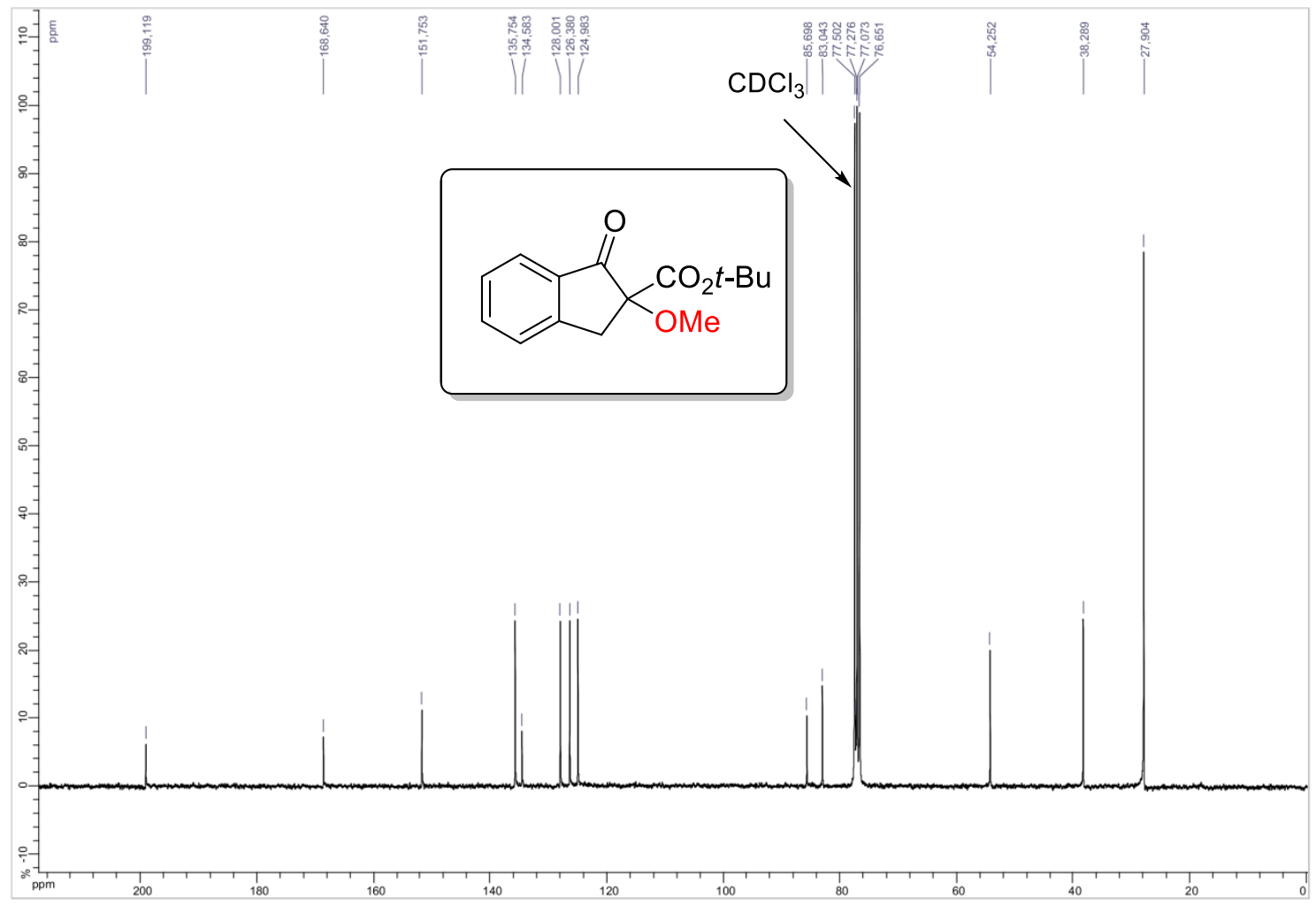


1-Methoxy-4-phenylbut-3-en-2-one 4u

${ }^{1} \mathrm{H}$ NMR (300 MHz, $\left.\mathrm{CDCl}_{3}\right)$

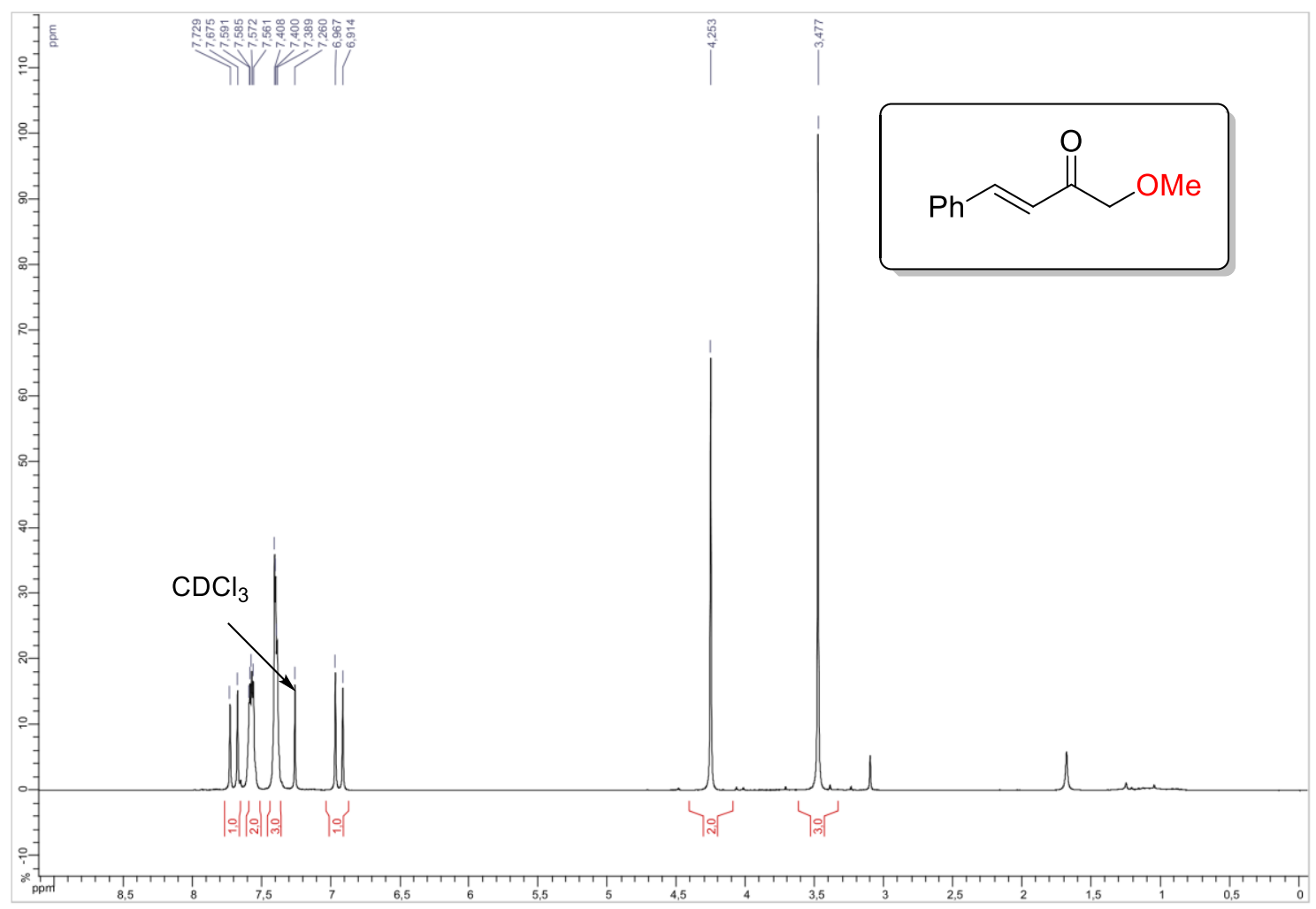

${ }^{13} \mathrm{C}$ NMR (75 MHz, CDCl3)

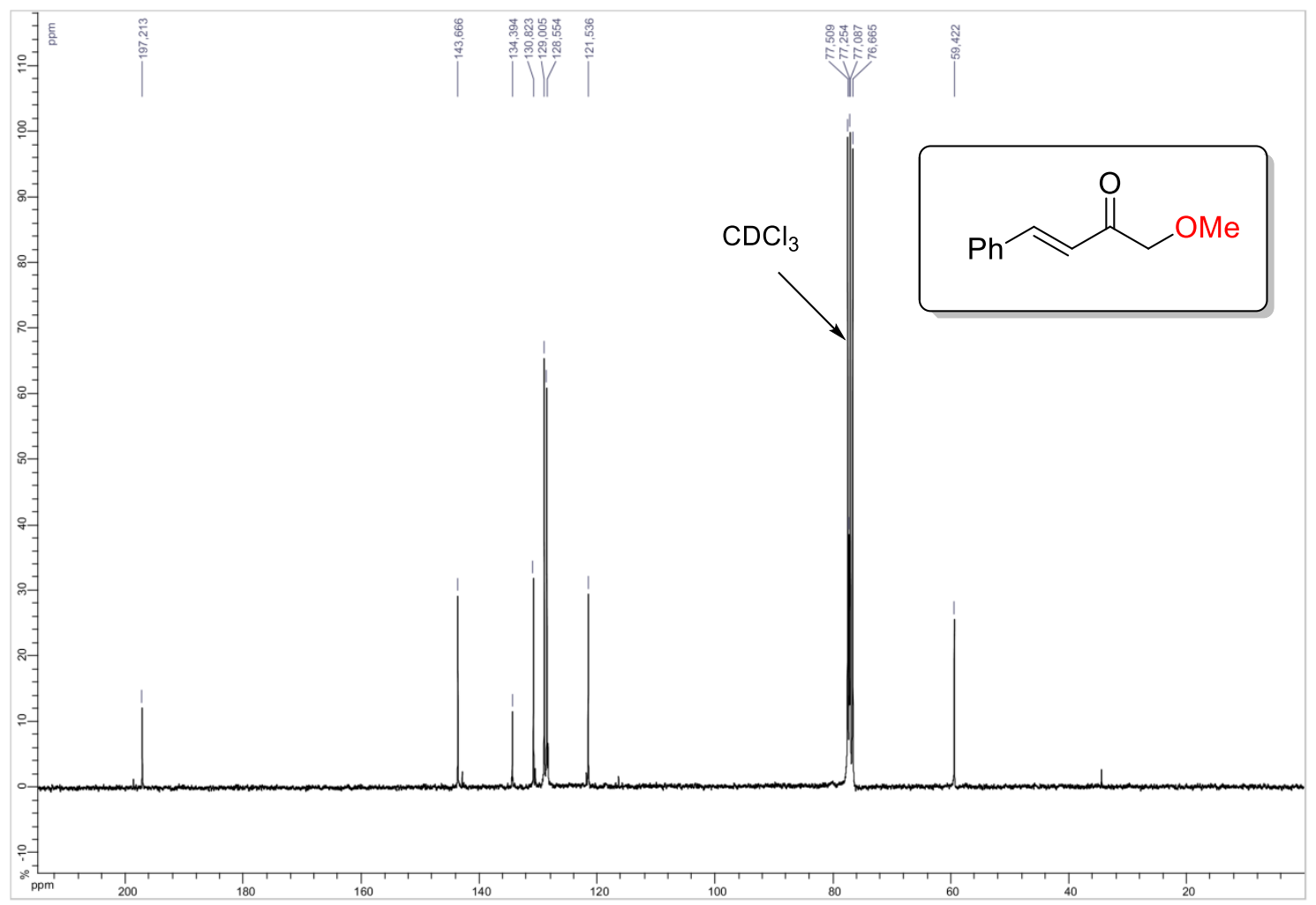


1-Methoxynon-3-en-2-one 4v

${ }^{1} \mathrm{H}$ NMR (300 MHz, CDCl 3$)$

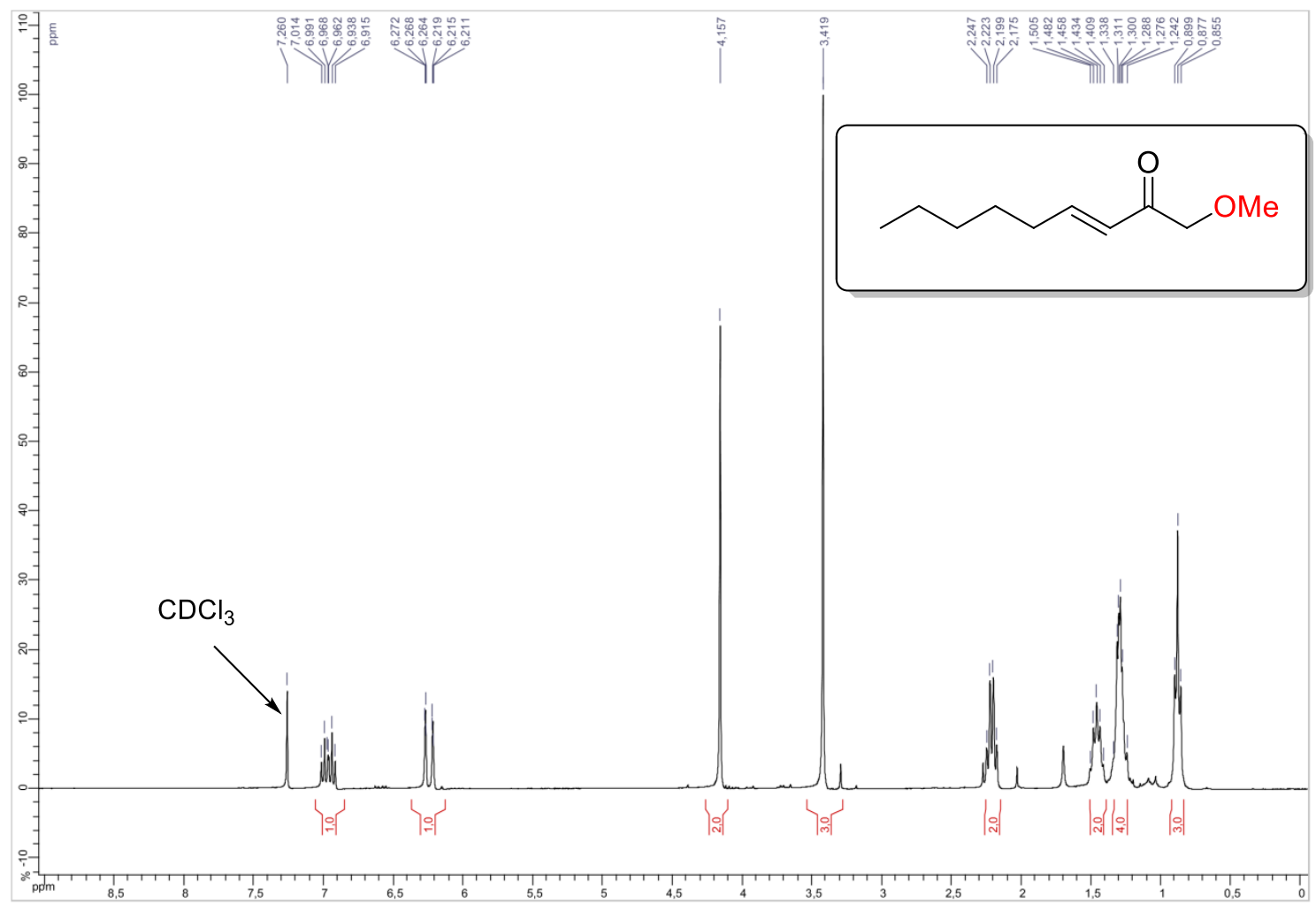

${ }^{13} \mathrm{C}$ NMR (75 MHz, CDCl3)

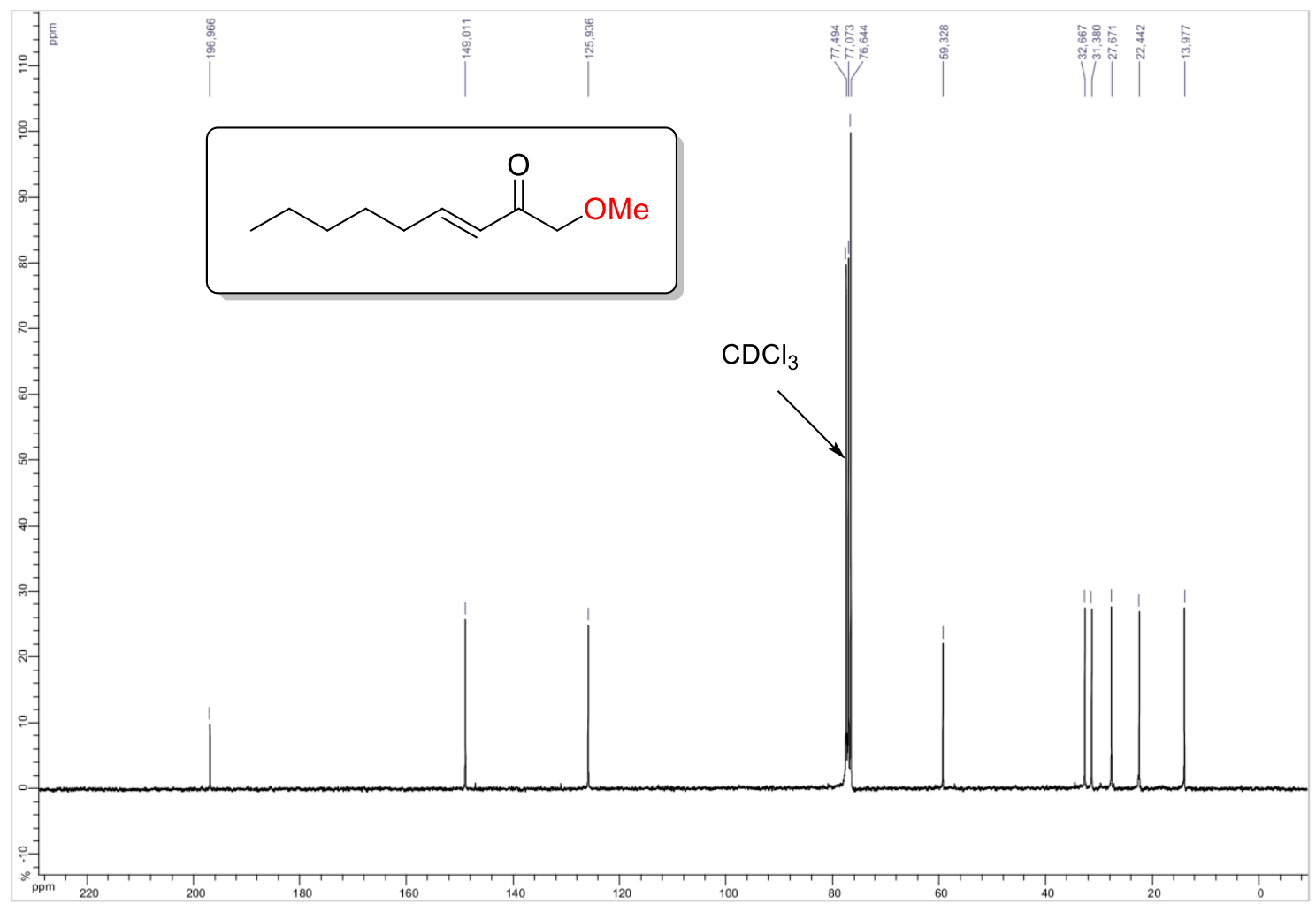


Cis-2-Methoxy-4-phenylcyclohexanone 4w

${ }^{1} \mathrm{H}$ NMR (300 MHz, $\left.\mathrm{CDCl}_{3}\right)$

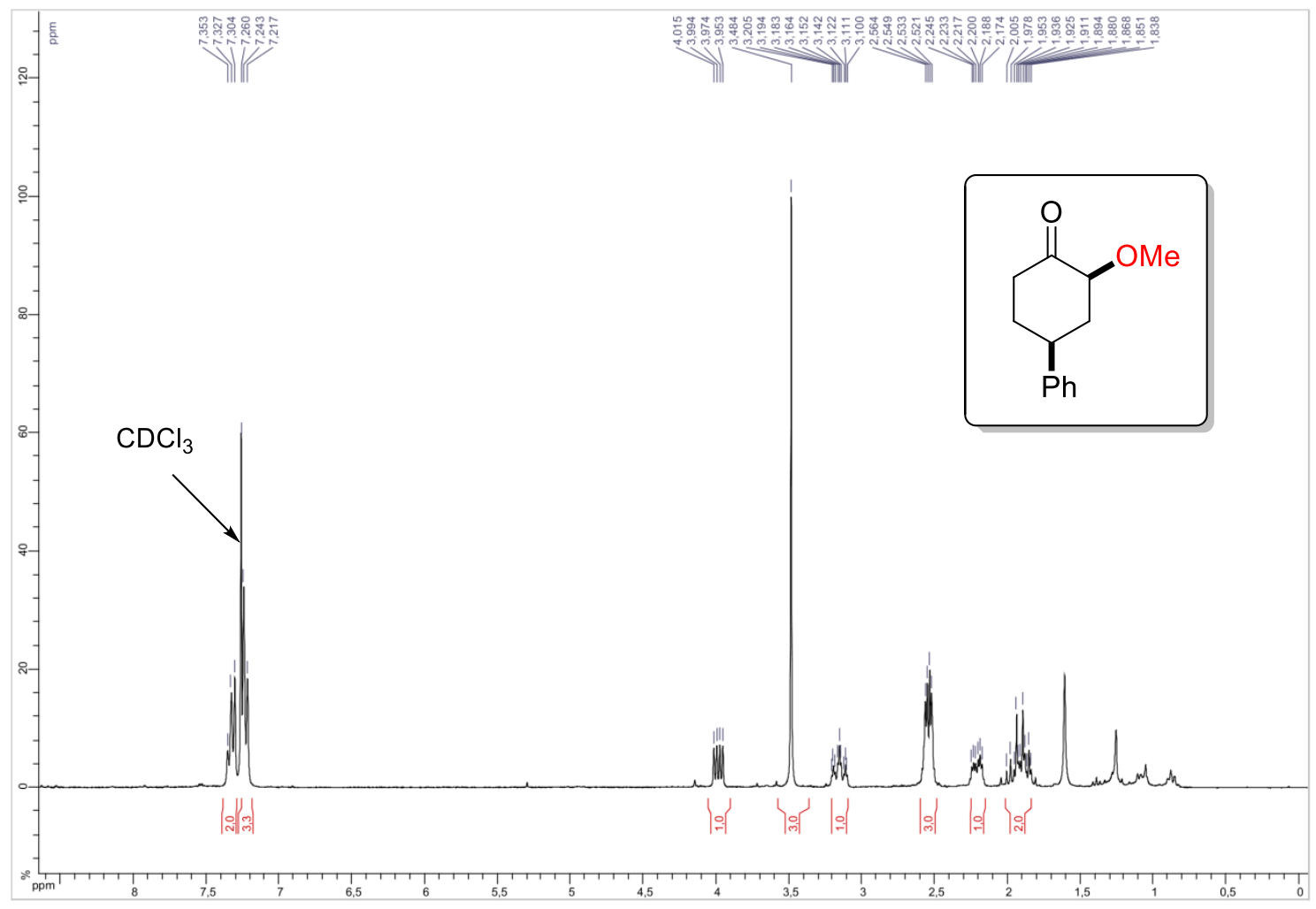

${ }^{13} \mathrm{C}$ NMR (75 MHz, CDCl3)

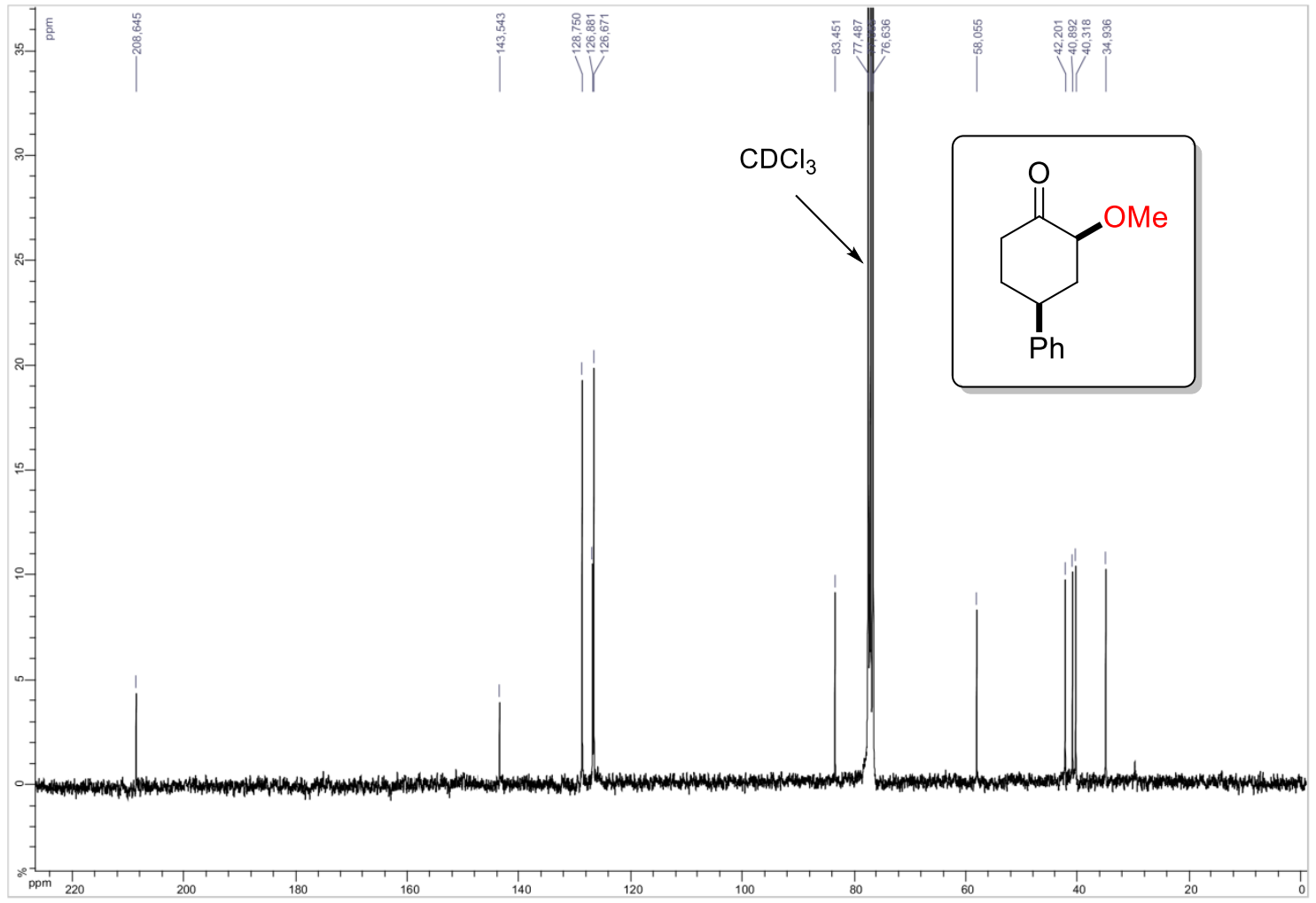


Cis-4-(Tert-butyl)-2-methoxycyclohexanone 4x

${ }^{1} \mathrm{H}$ NMR (300 MHz, $\left.\mathrm{CDCl}_{3}\right)$

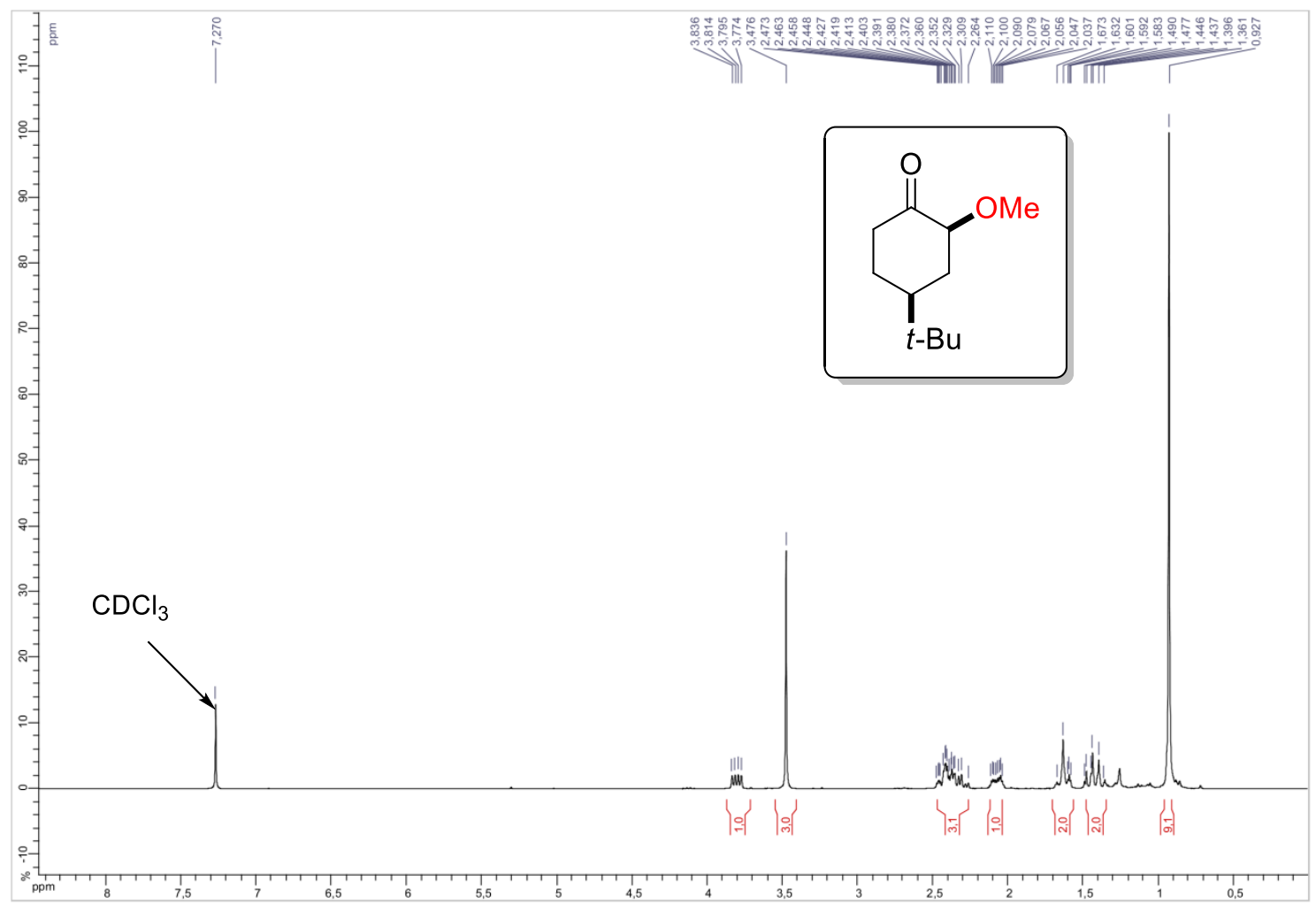

${ }^{13} \mathrm{C}$ NMR (75 MHz, CDCl3)

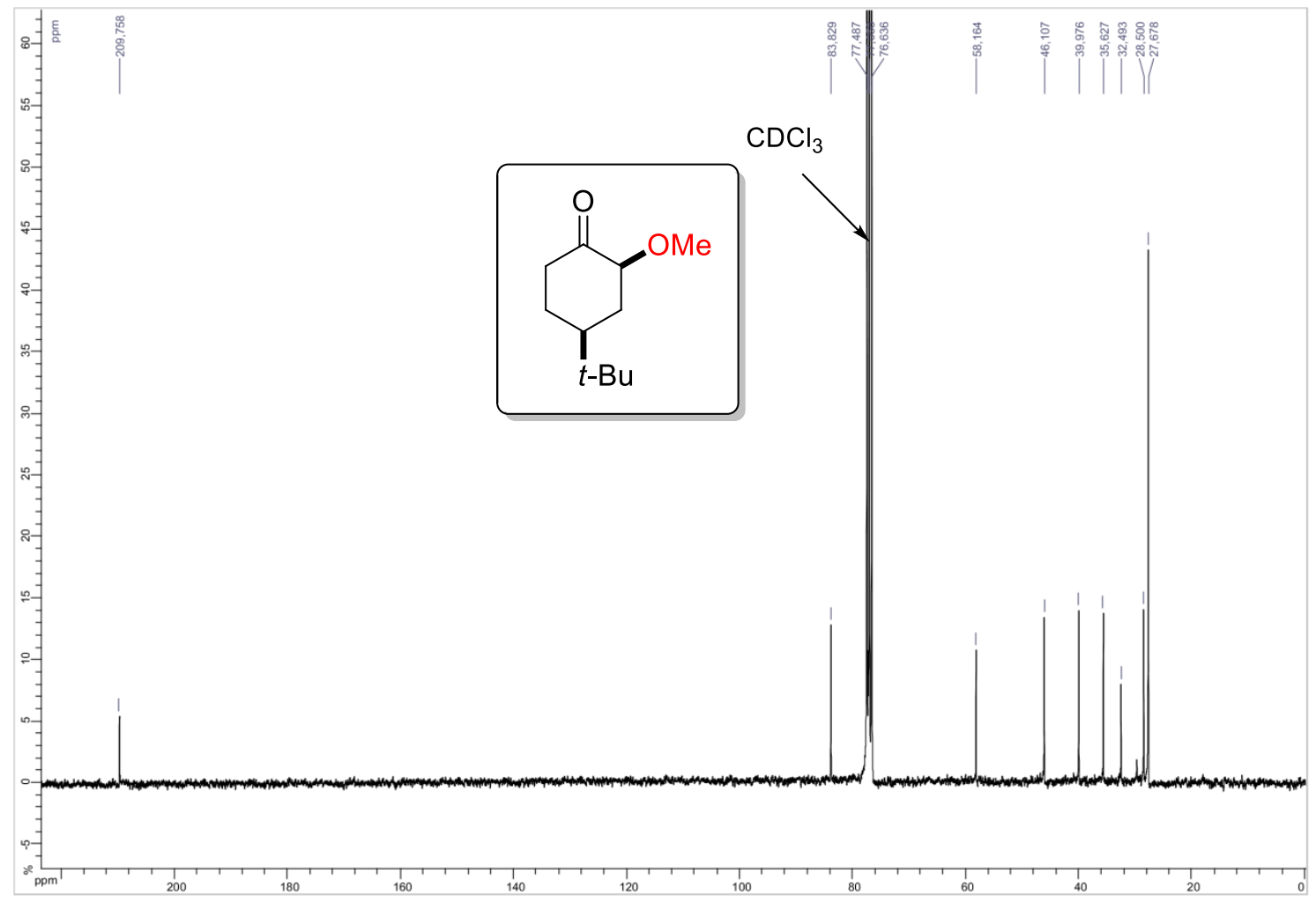


5-Methoxyundecan-6-one 4y

${ }^{1} \mathrm{H}$ NMR (300 MHz, $\left.\mathrm{CDCl}_{3}\right)$

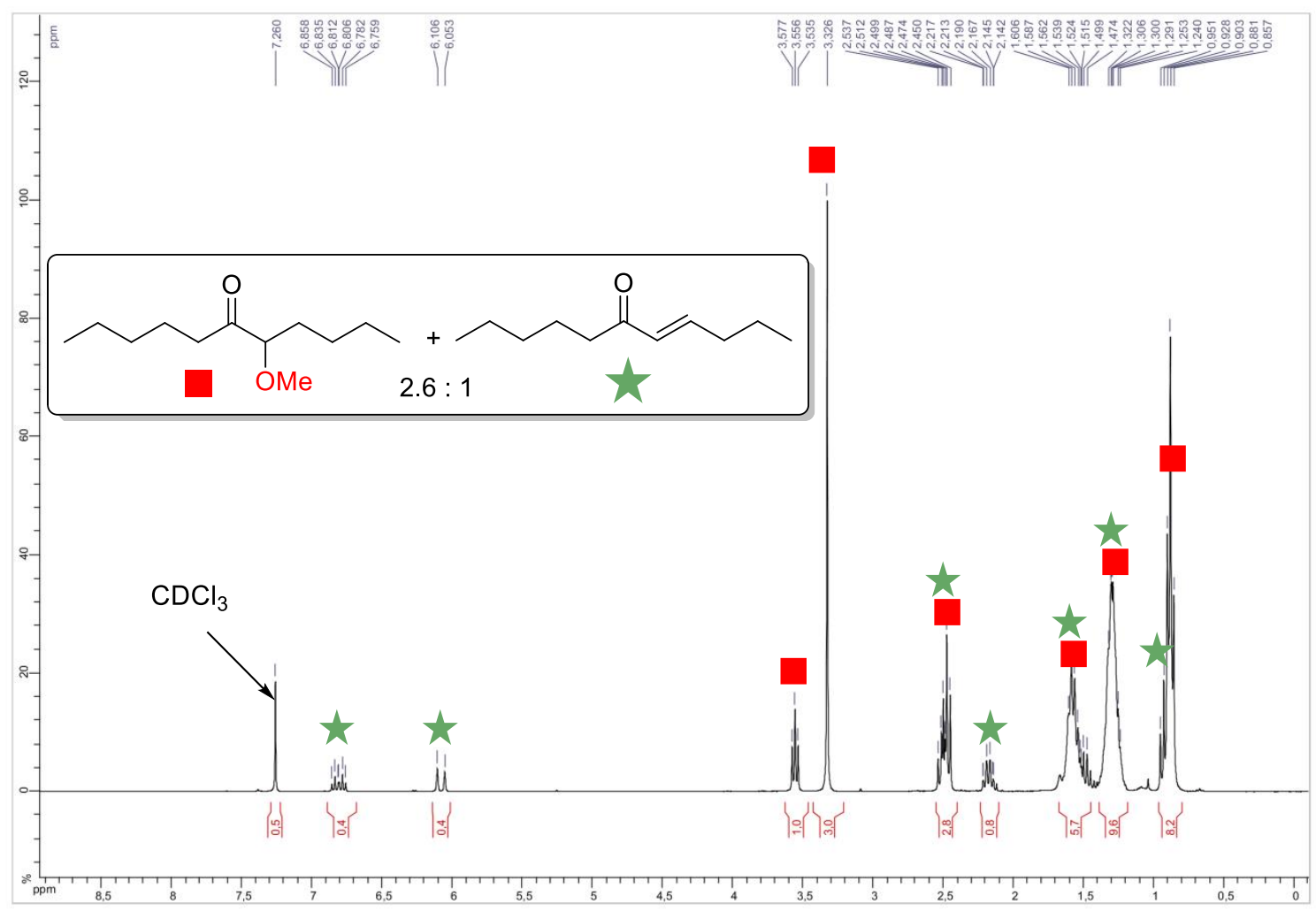

${ }^{13} \mathrm{C}$ NMR (75 MHz, CDCl3)

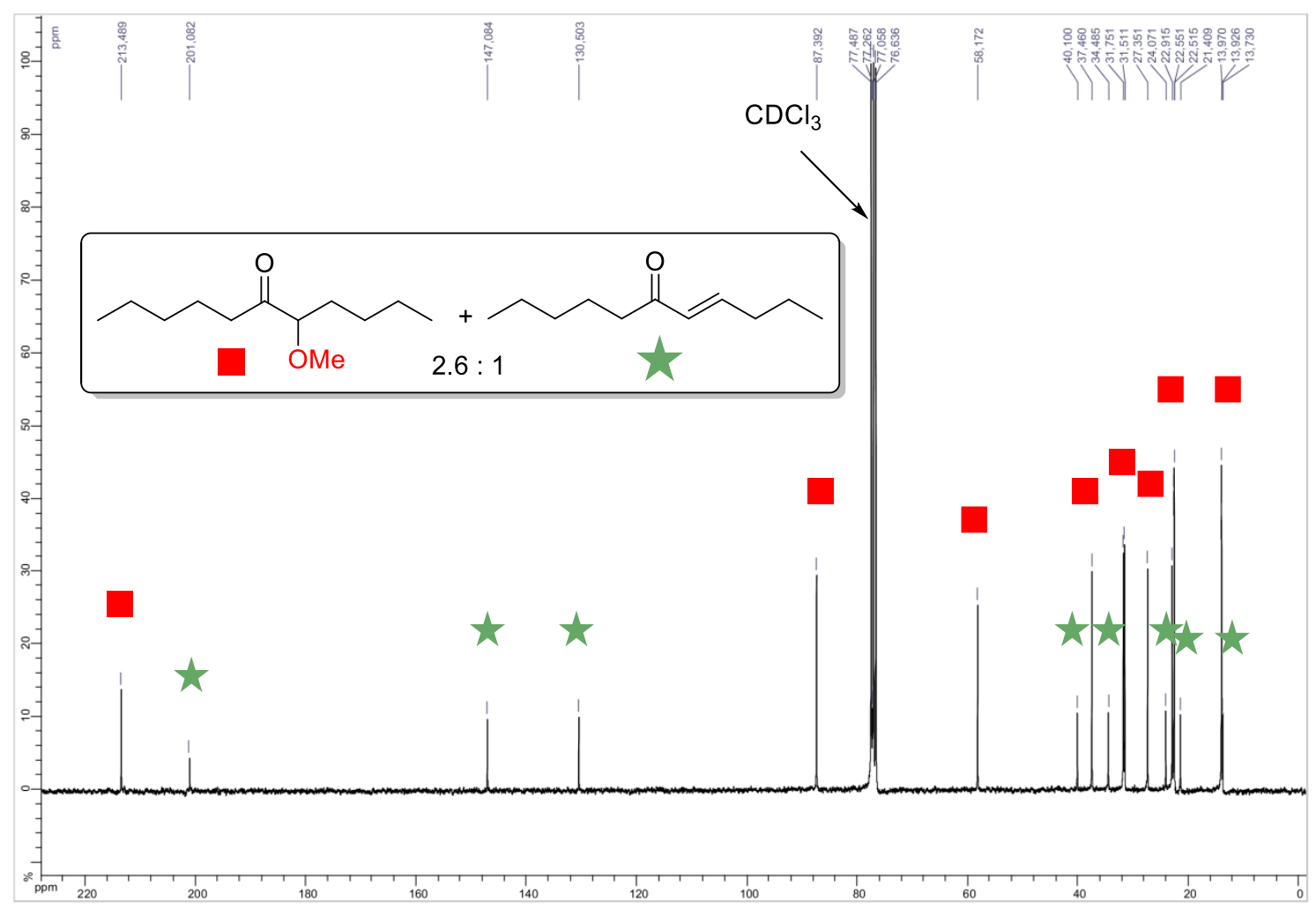


1-(6-(tert-butyl)-1,1-dimethyl-2,3-dihydro-1H-inden-4-yl)-2-methoxyethanone 4z ${ }^{1} \mathrm{H}$ NMR (300 MHz, $\left.\mathrm{CDCl}_{3}\right)$

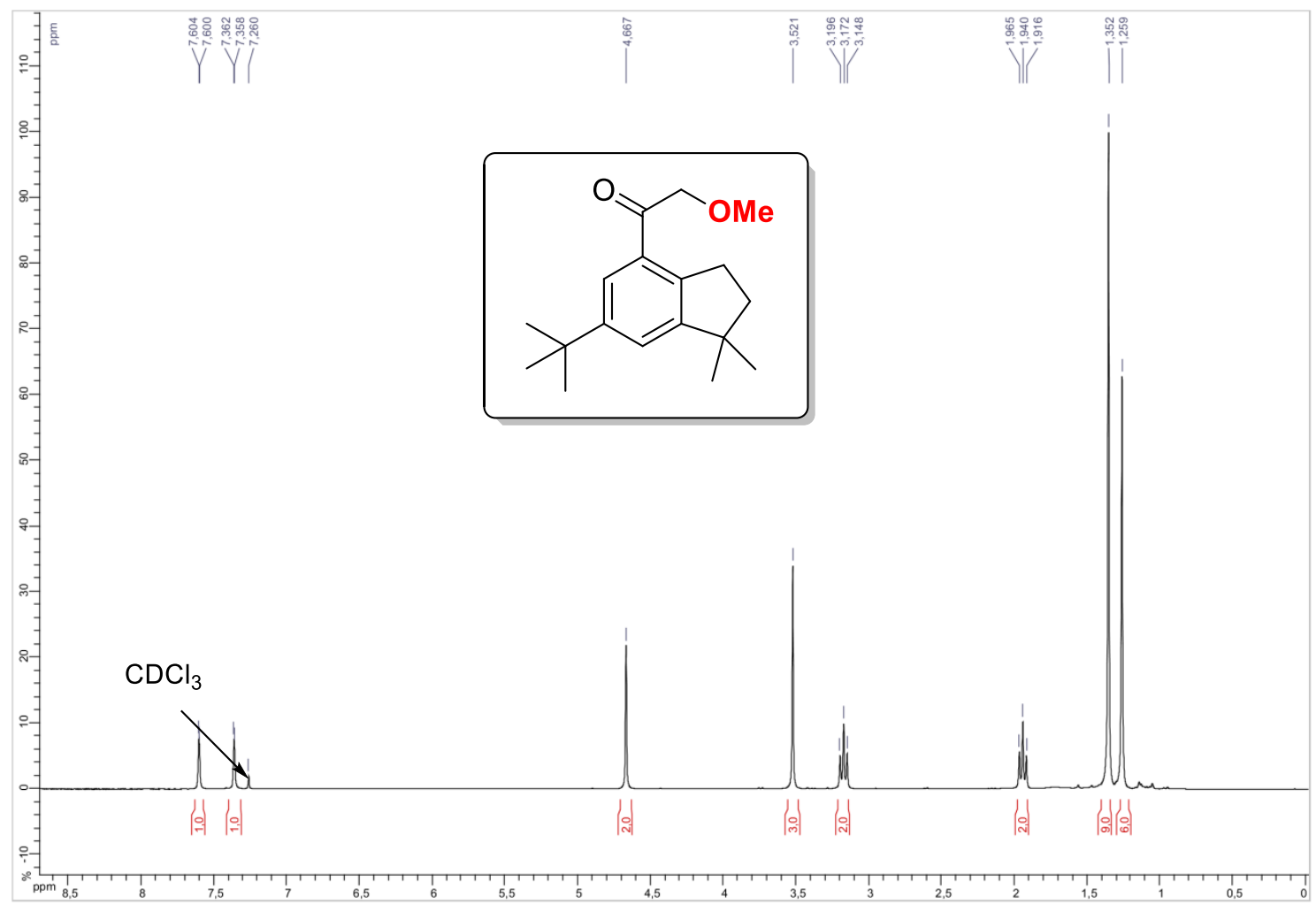

${ }^{13} \mathrm{C}$ NMR (75 MHz, $\left.\mathrm{CDCl}_{3}\right)$

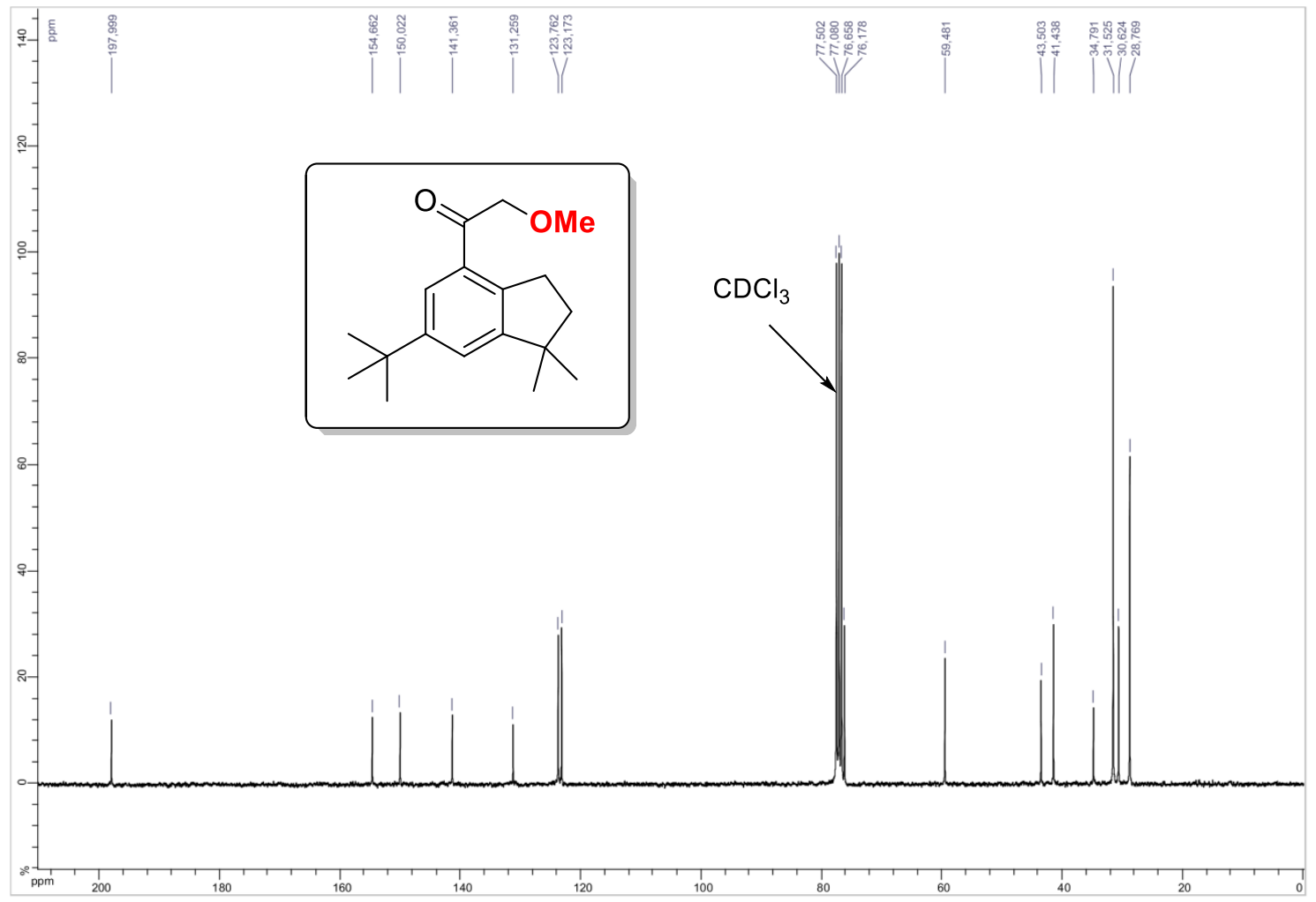


1-(1,1,2,3,3,6-hexamethyl-2,3-dihydro-1H-inden-5-yl)-2-methoxyethanone 4aa ${ }^{1} \mathrm{H}$ NMR (300 MHz, $\left.\mathrm{CDCl}_{3}\right)$

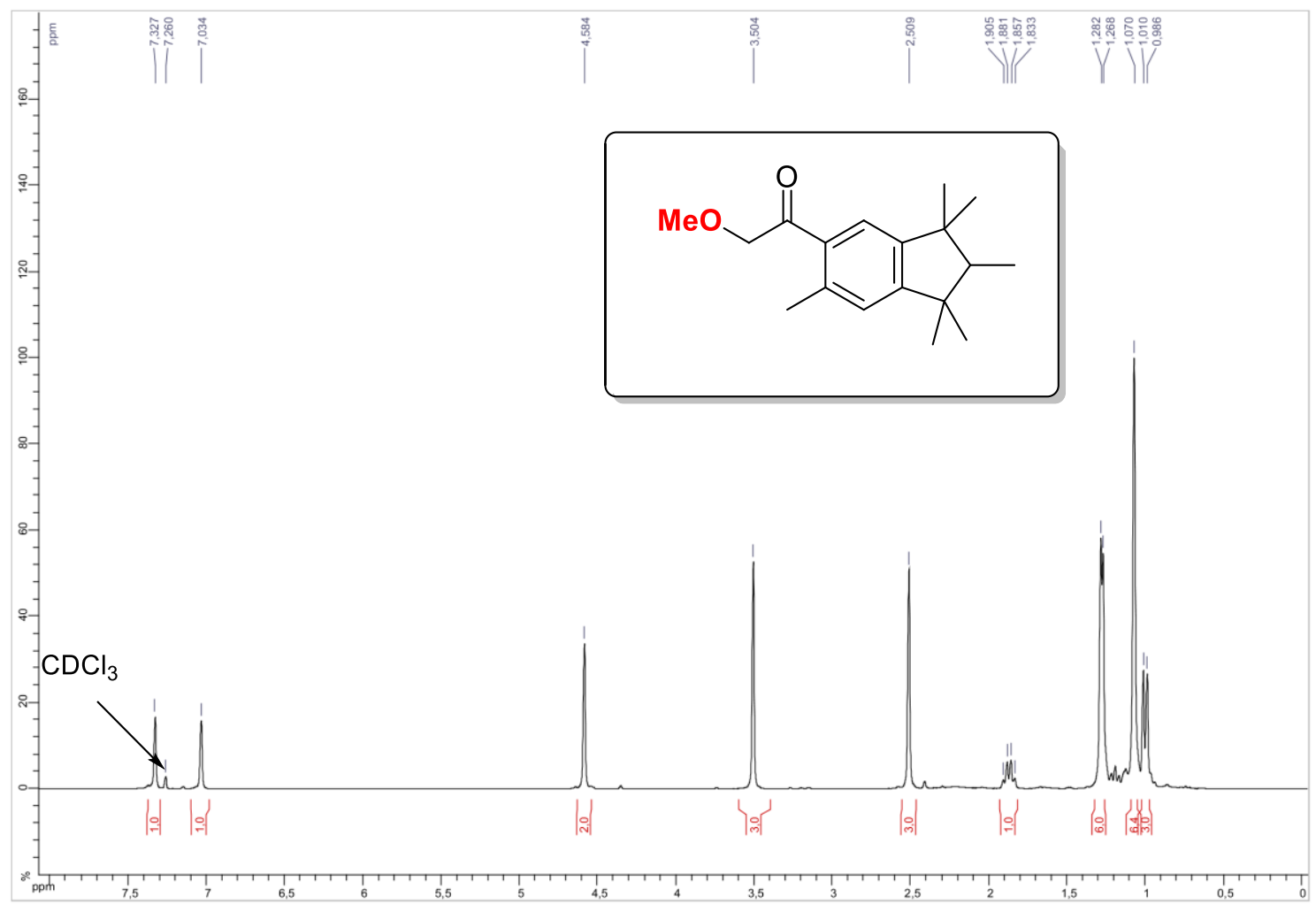

${ }^{13} \mathrm{C}$ NMR (75 MHz, $\left.\mathrm{CDCl}_{3}\right)$

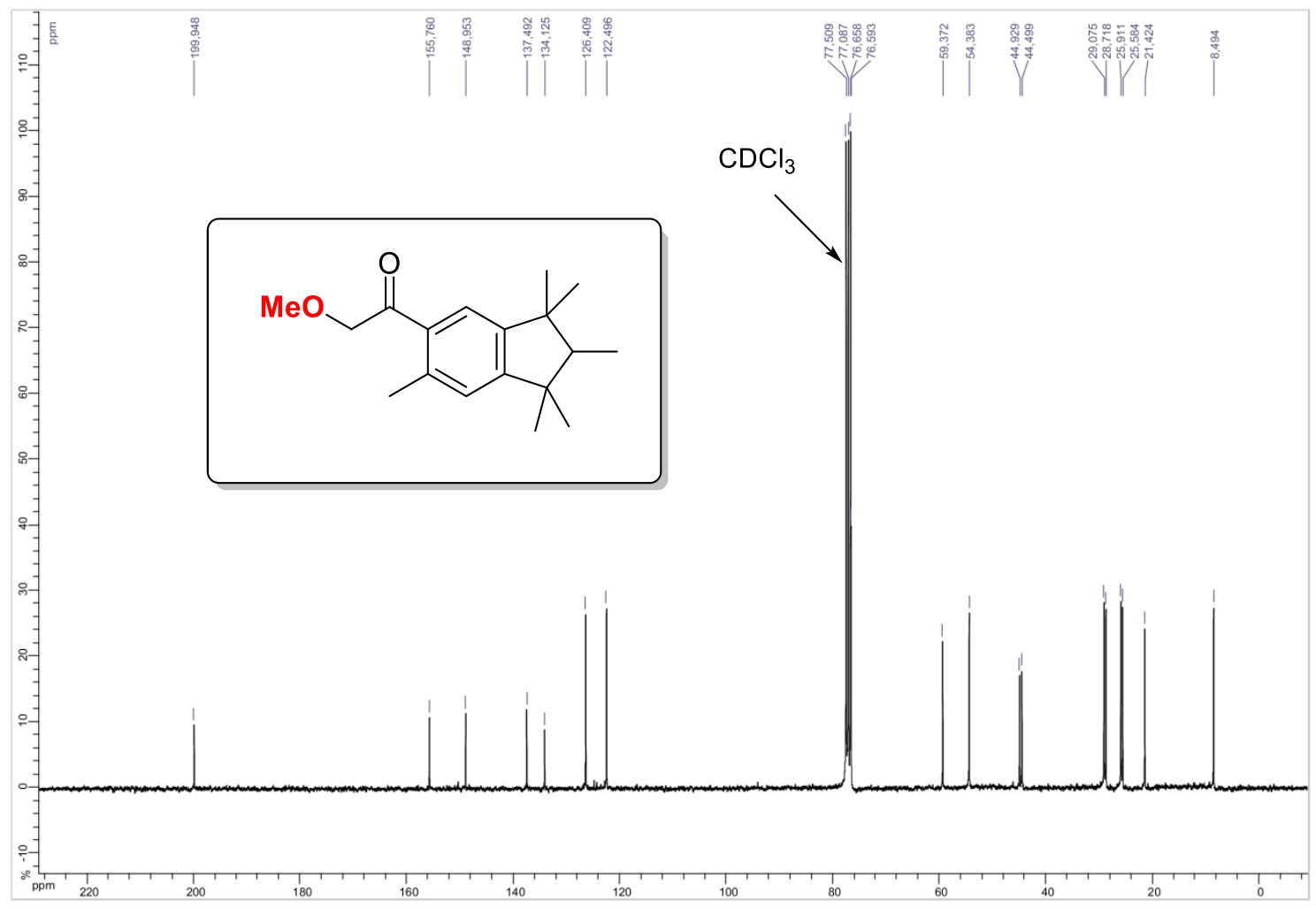


1-Methoxy-4-(2,6,6-trimethylcyclohex-2-en-1-yl)but-3-en-2-one 4ab ${ }^{1} \mathrm{H}$ NMR (300 MHz, $\left.\mathrm{CDCl}_{3}\right)$

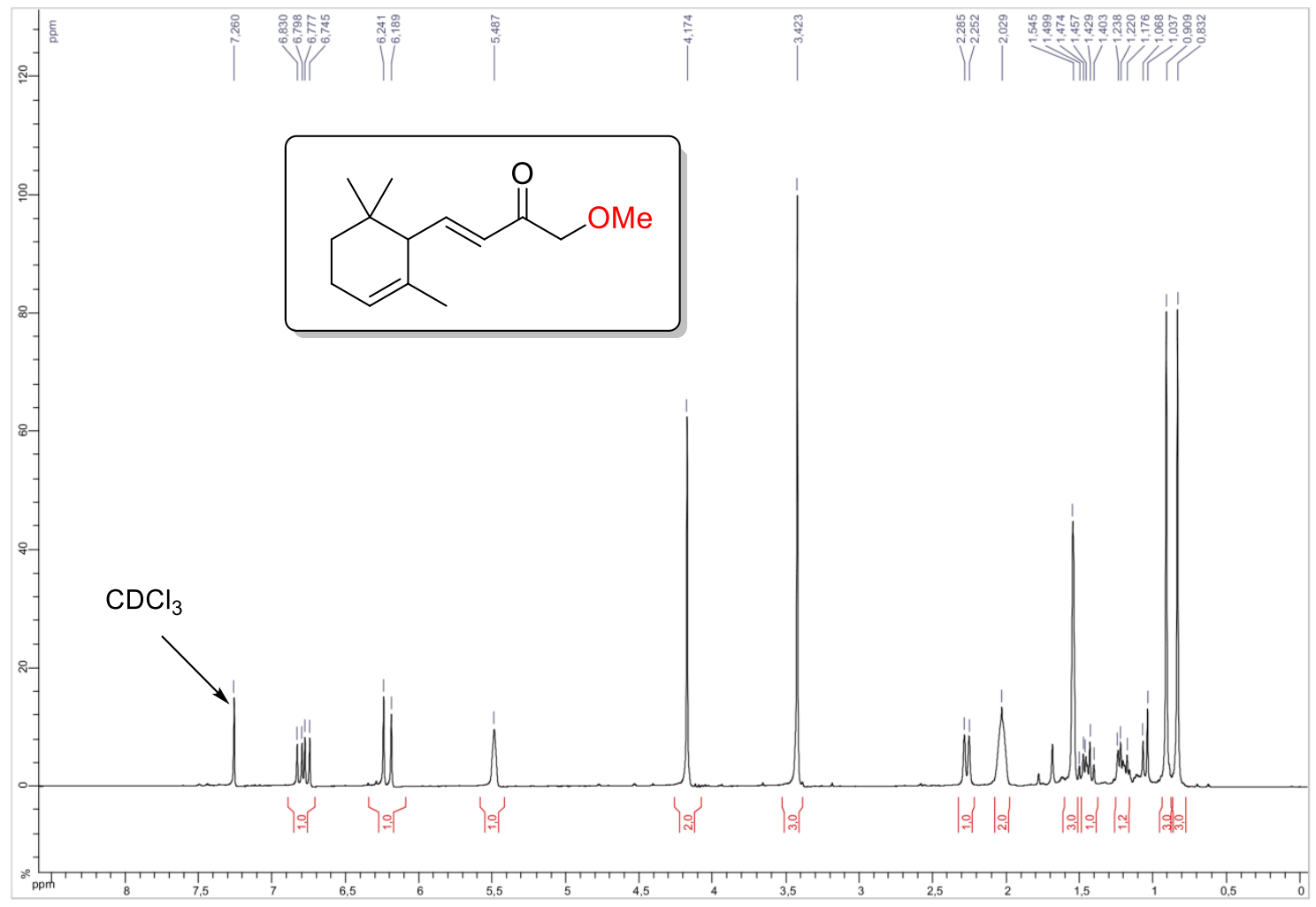

${ }^{13} \mathrm{C}$ NMR (75 MHz, CDCl3)

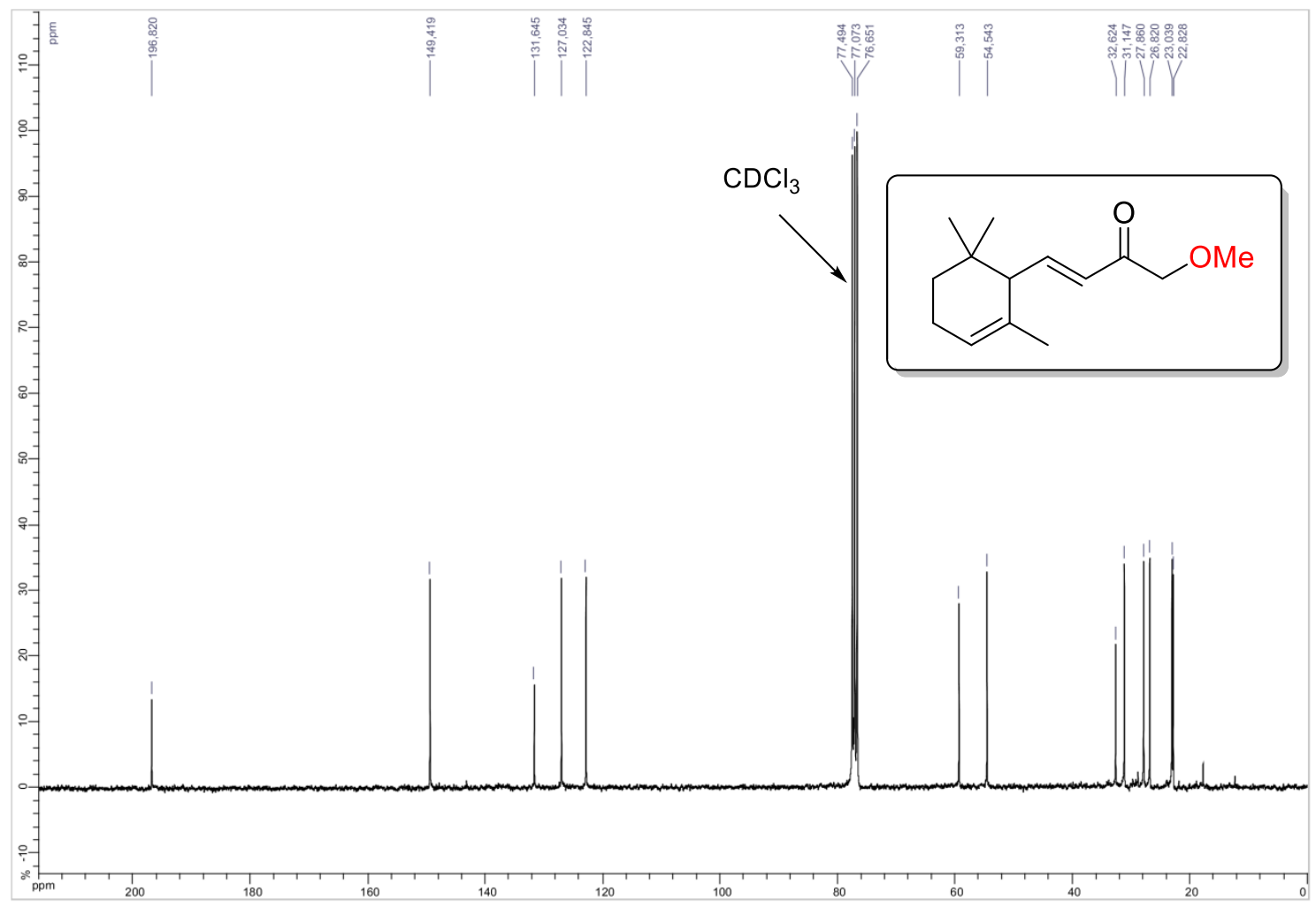


(S)-4-(2-methoxyacetyl)phenyl 2-((tert-butoxycarbonyl)amino)propanoate 4ac ${ }^{1} \mathrm{H}$ NMR (300 MHz, CDCl 3 )

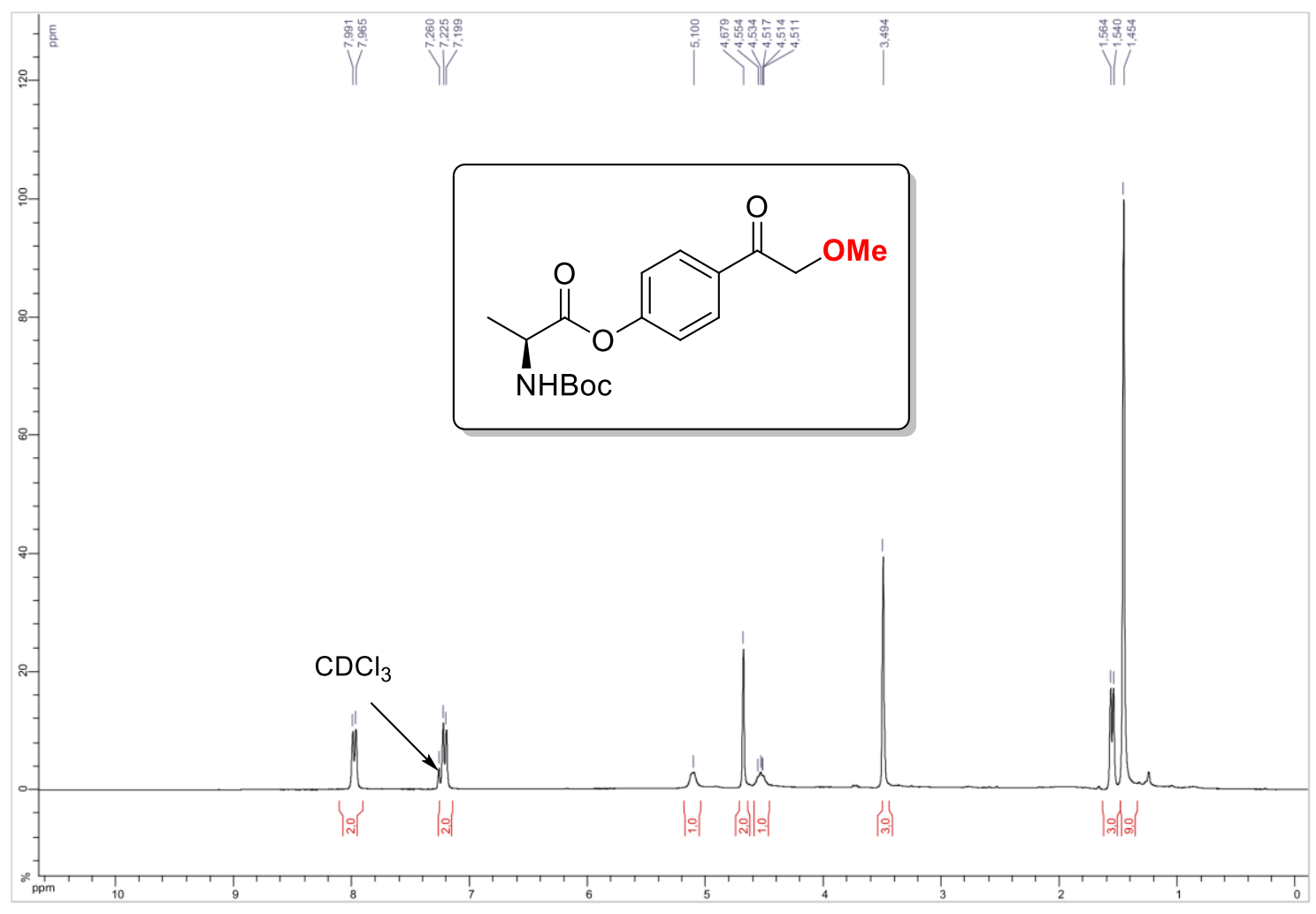

${ }^{13} \mathrm{C}$ NMR (75 MHz, CDCl3)

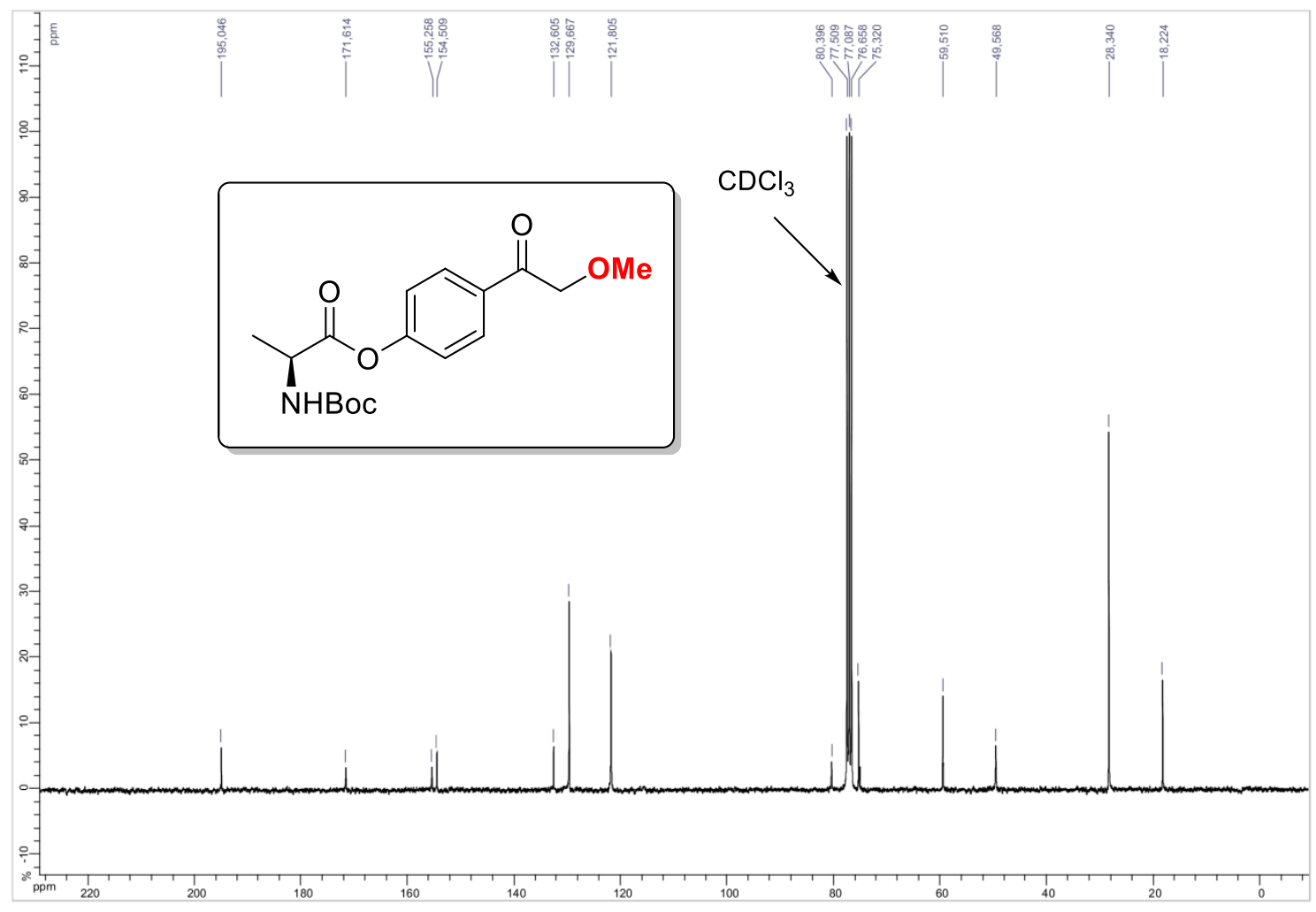


6,17-Dimethoxy-10,13-dimethyl-6,7,8,9,10,11,12,13,14,15,16,17-dodecahydro-1Hcyclopenta[a]phenanthren-3(2H)-one $4 \mathrm{ad}$

${ }^{1} \mathrm{H}$ NMR (300 MHz, $\mathrm{CDCl}_{3}$ )

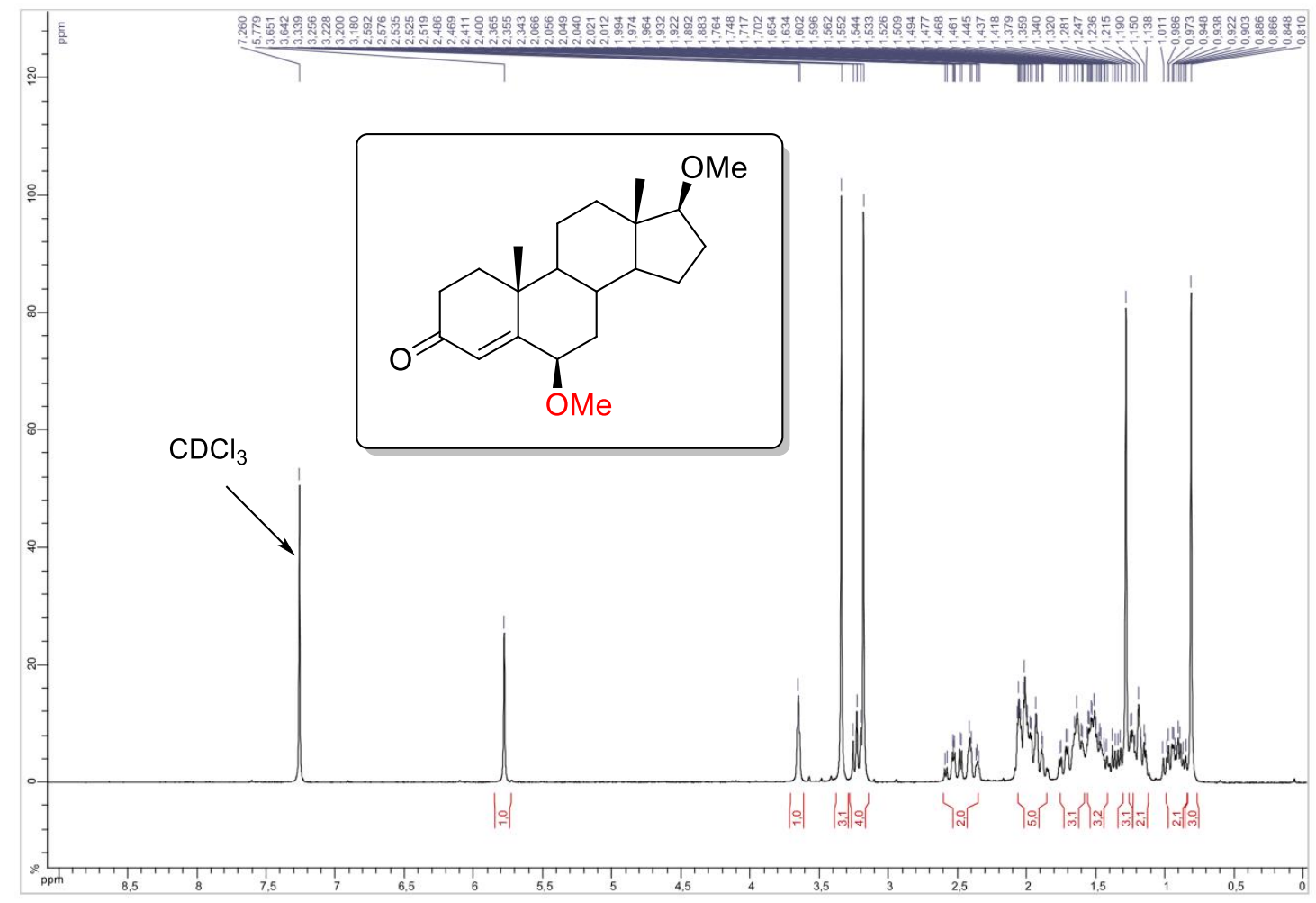

${ }^{13} \mathrm{C}$ NMR (75 MHz, $\left.\mathrm{CDCl}_{3}\right)$

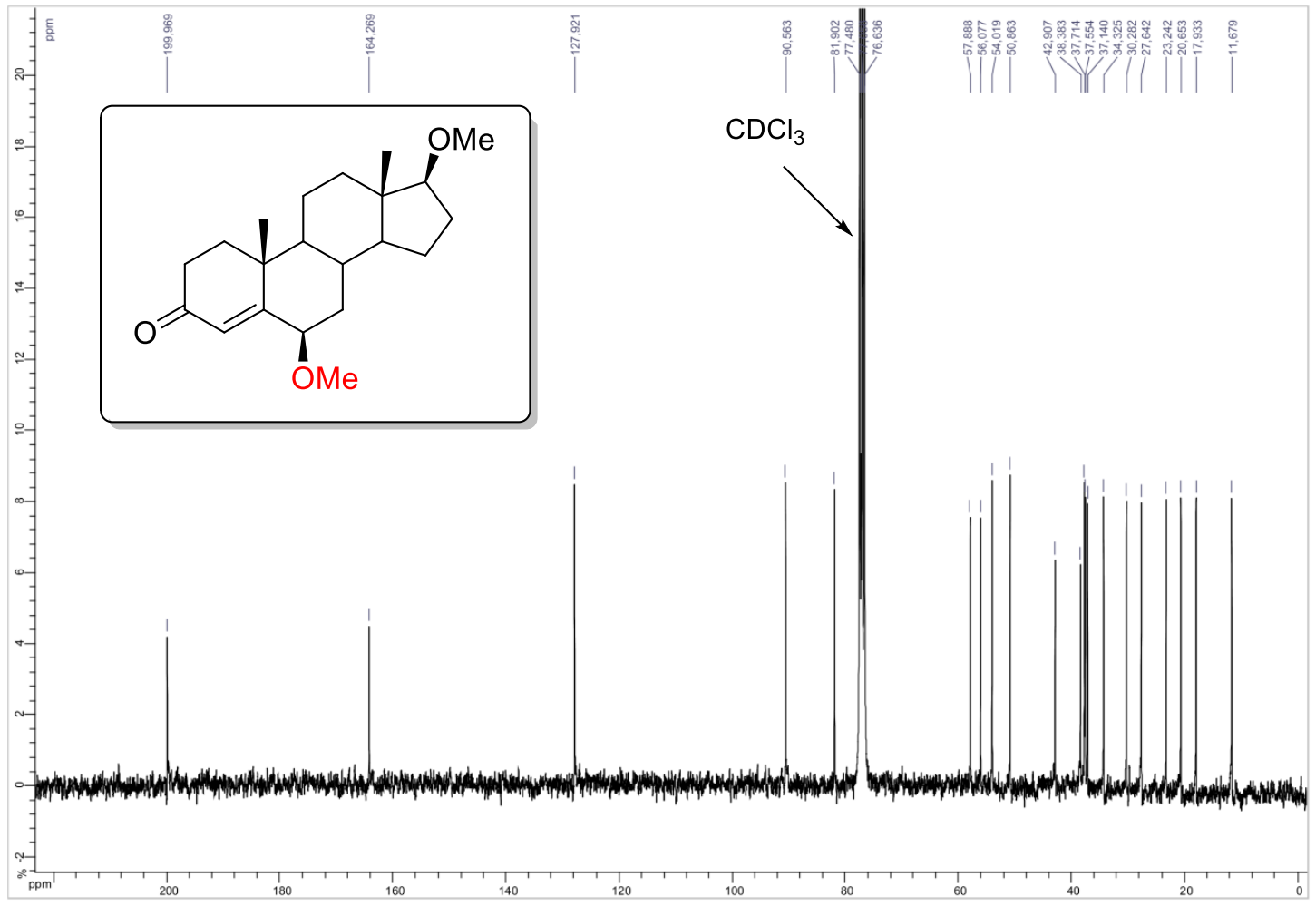


6,17-Dimethoxy-10,13-dimethyl-6,7,8,9,10,11,12,13,14,15,16,17-dodecahydro-1Hcyclopenta[a]phenanthren-3(2H)-one 4ad

${ }^{1}$ H NMR (300 MHz, CDCl3)

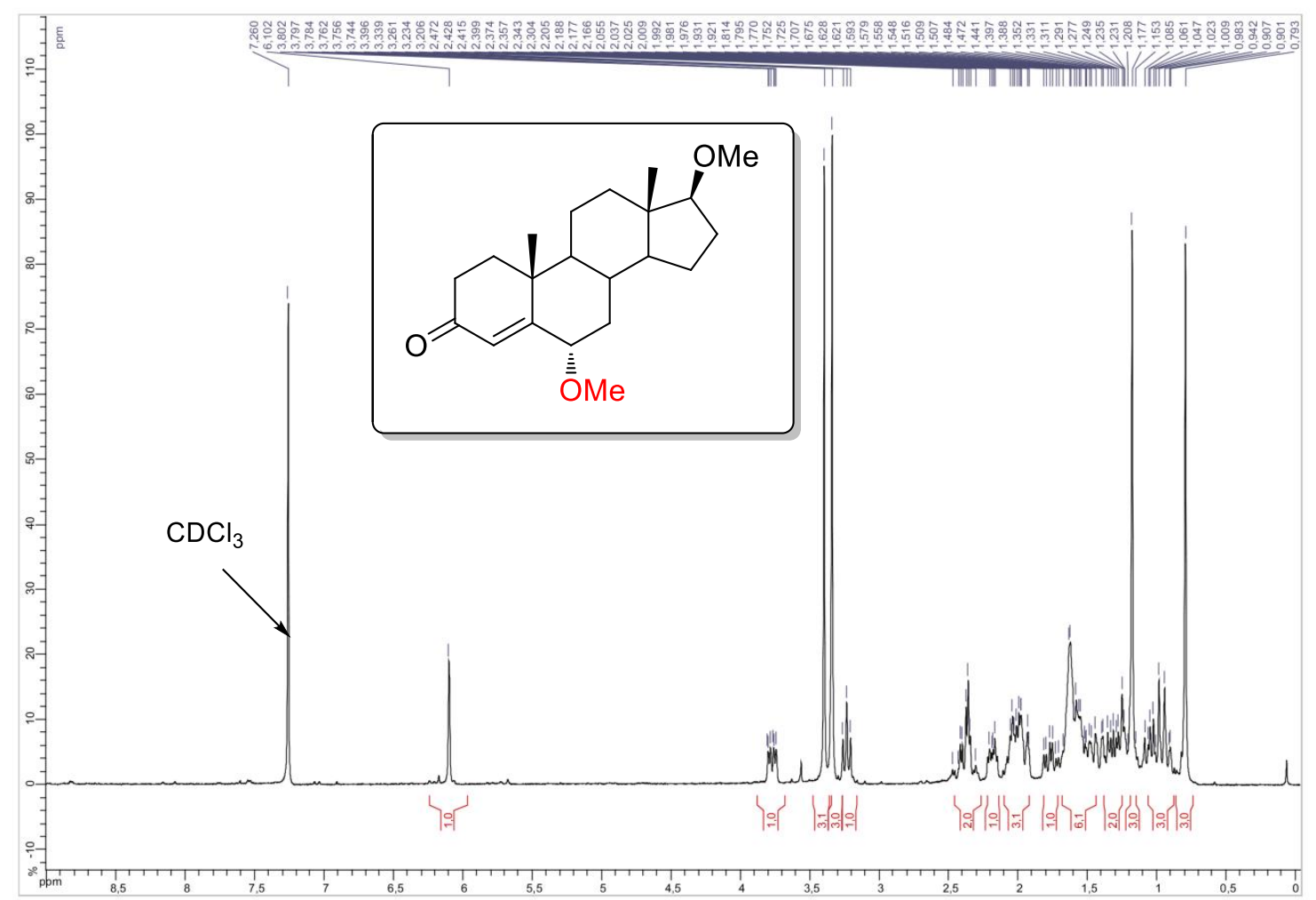

${ }^{13} \mathrm{C}$ NMR (75 $\left.\mathrm{MHz}, \mathrm{CDCl}_{3}\right)$

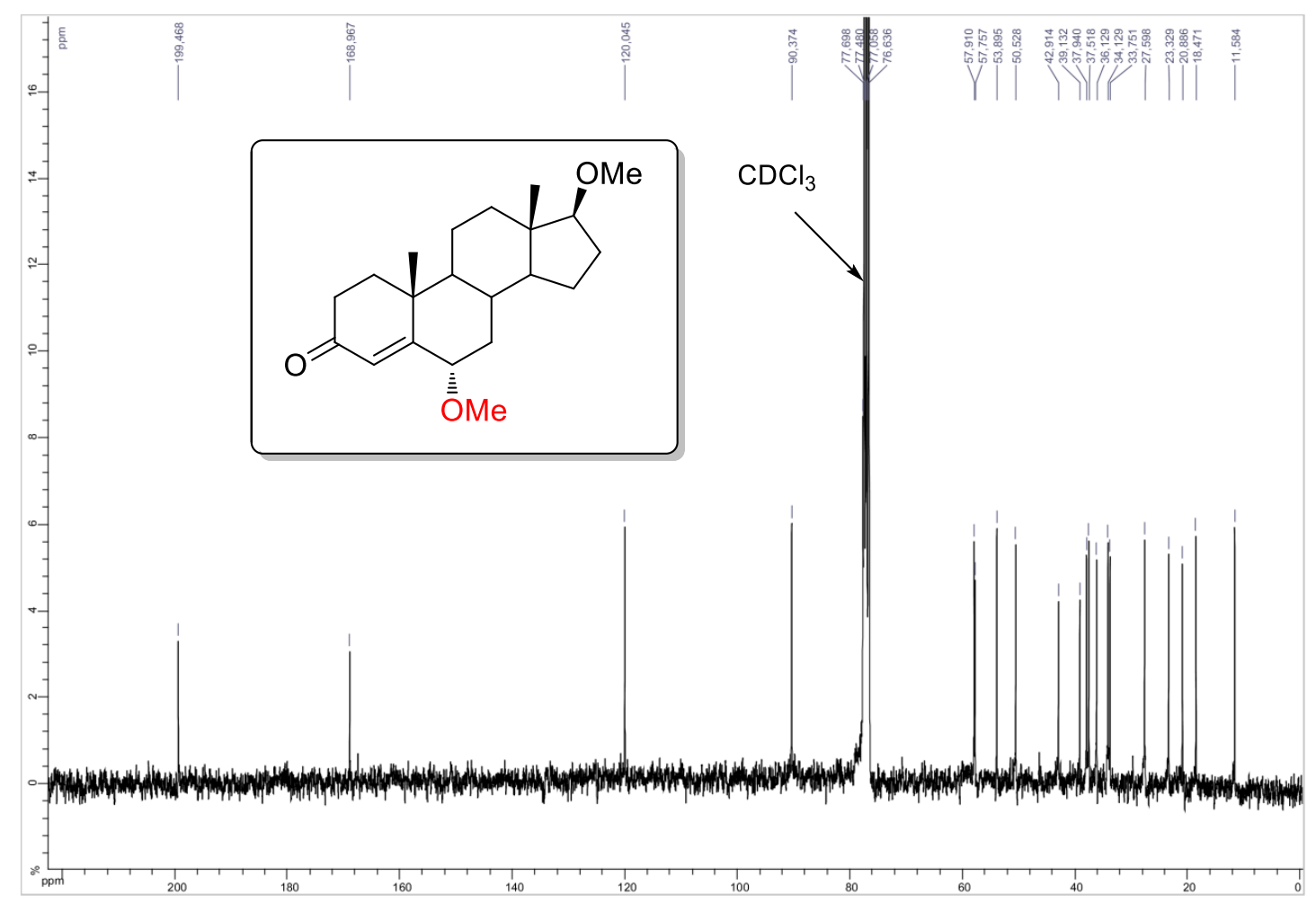


8-Methoxy-4,4a-dimethyl-6-(prop-1-en-2-yl)-4,4a,5,6,7,8-hexahydronaphthalen-2(3H)one 4ae

${ }^{1} \mathrm{H}$ NMR (300 MHz, $\left.\mathrm{CDCl}_{3}\right)$

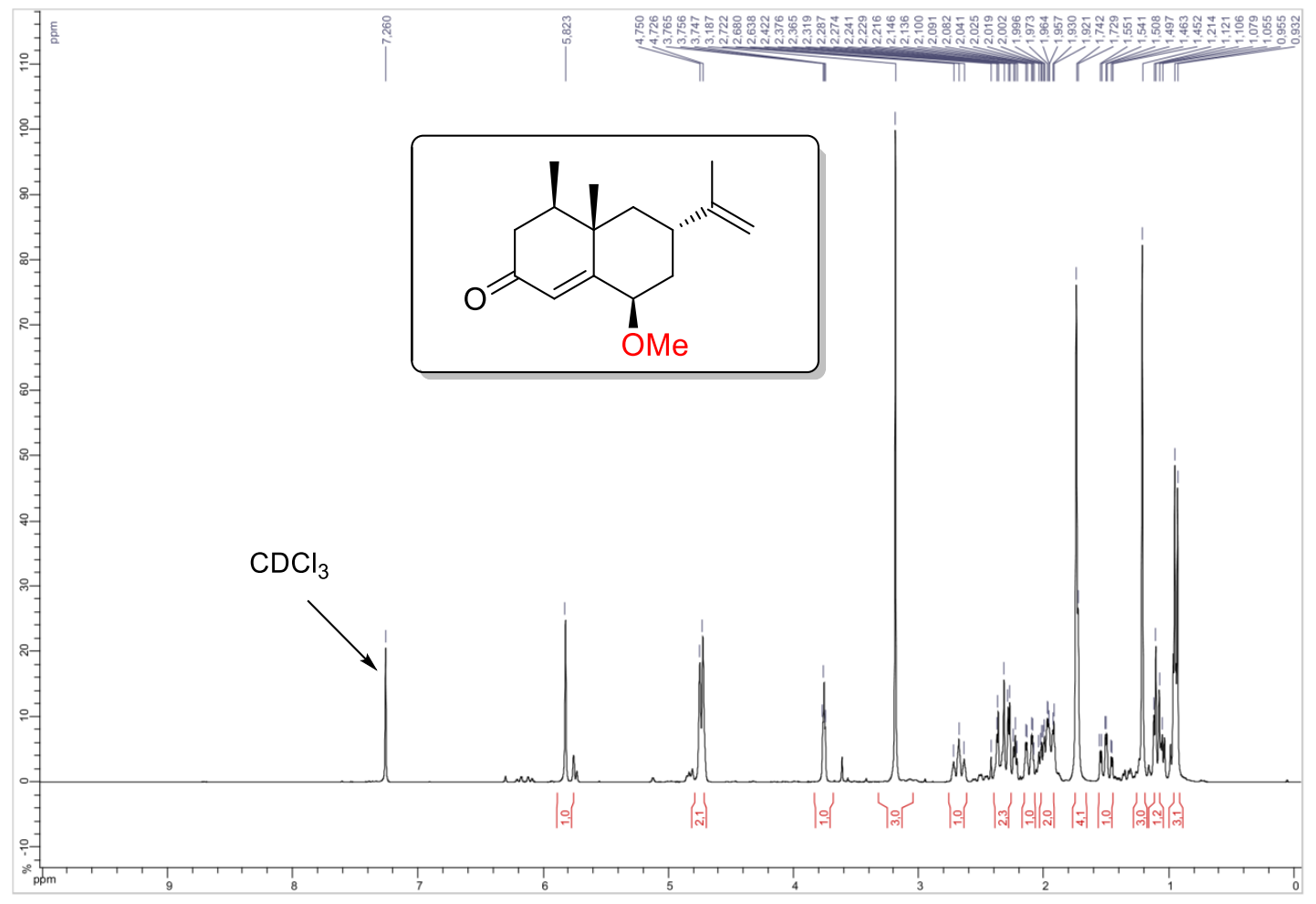

${ }^{13} \mathrm{C}$ NMR (75 MHz, CDCl3)

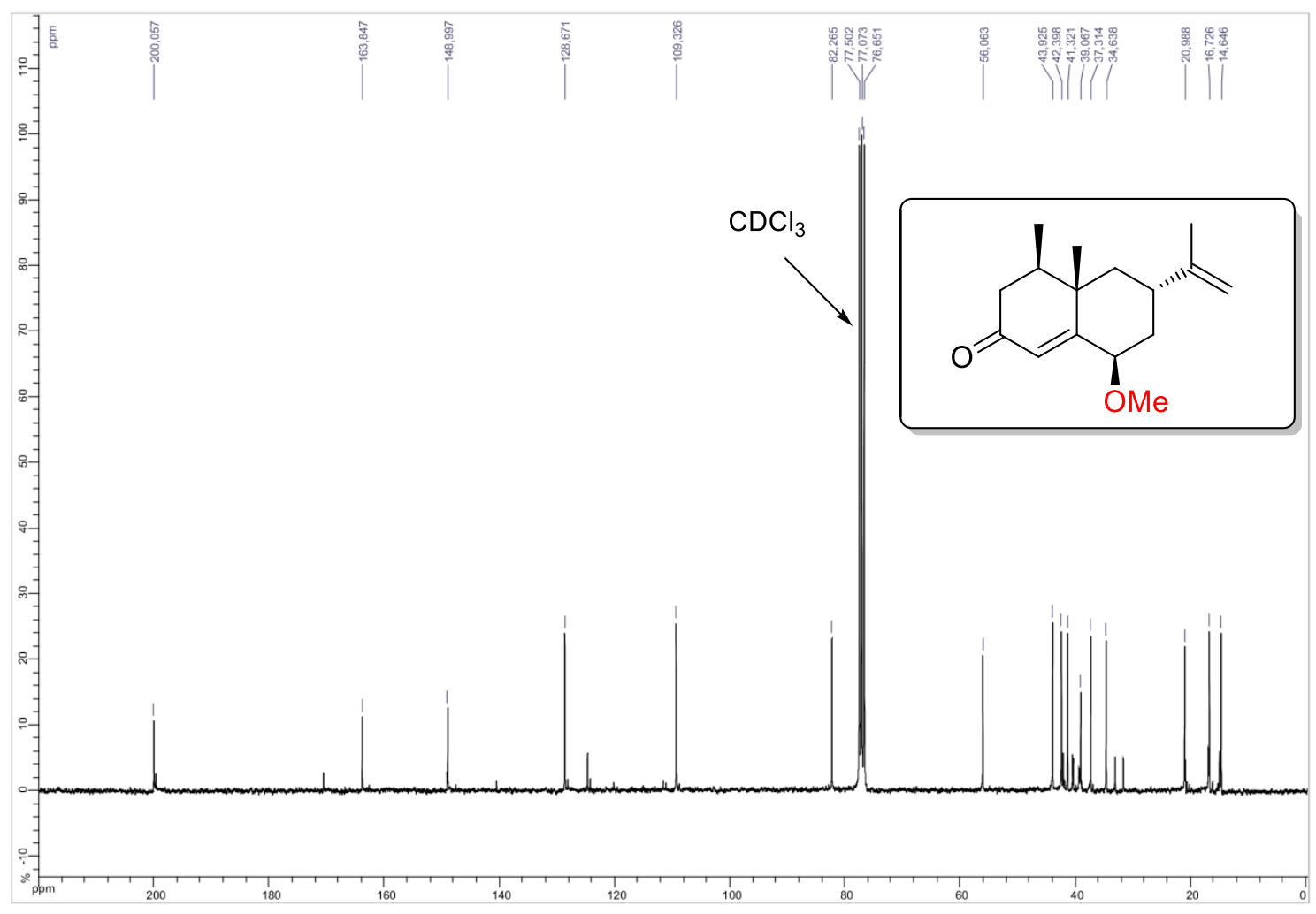


8-Methoxy-4,4a-dimethyl-6-(prop-1-en-2-yl)-4,4a,5,6,7,8-hexahydronaphthalen-2(3H)one 4ae

${ }^{1} \mathrm{H}$ NMR (300 MHz, CDCl3)

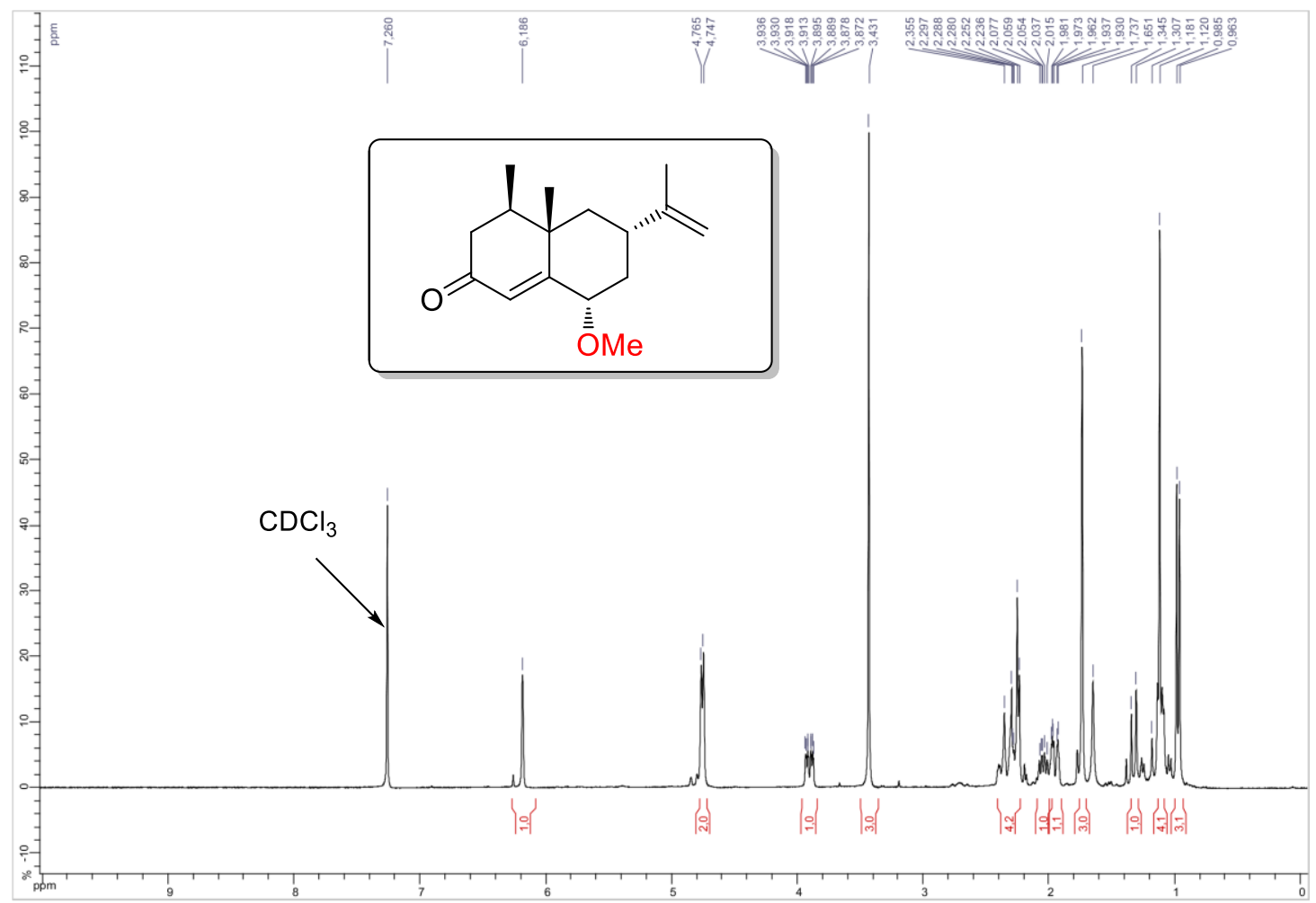

${ }^{13} \mathrm{C}$ NMR (75 MHz, $\left.\mathrm{CDCl}_{3}\right)$

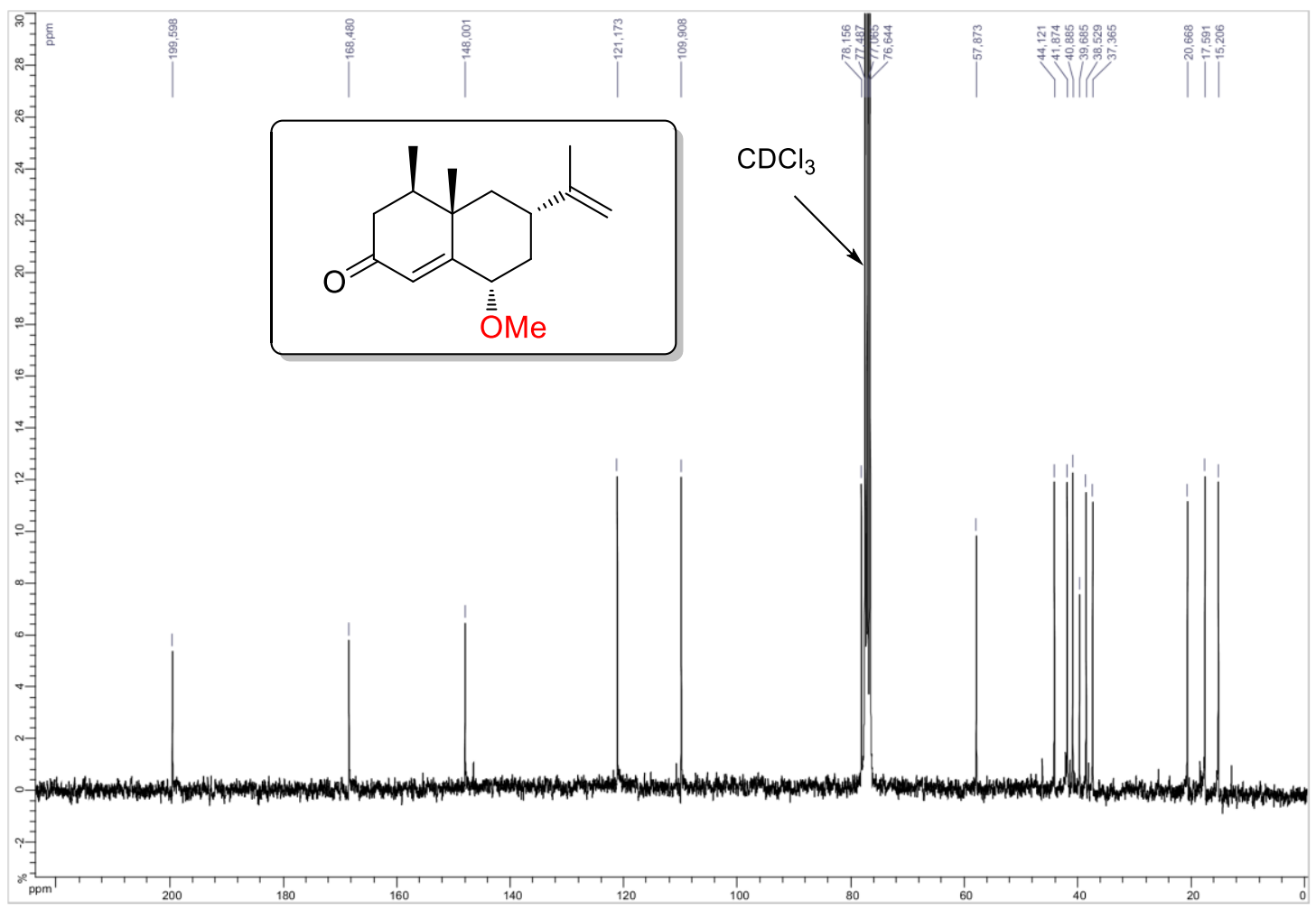


2-Ethoxy-1-(4-fluorophenyl)ethanone 4af

${ }^{1} \mathrm{H}$ NMR (300 MHz, $\left.\mathrm{CDCl}_{3}\right)$

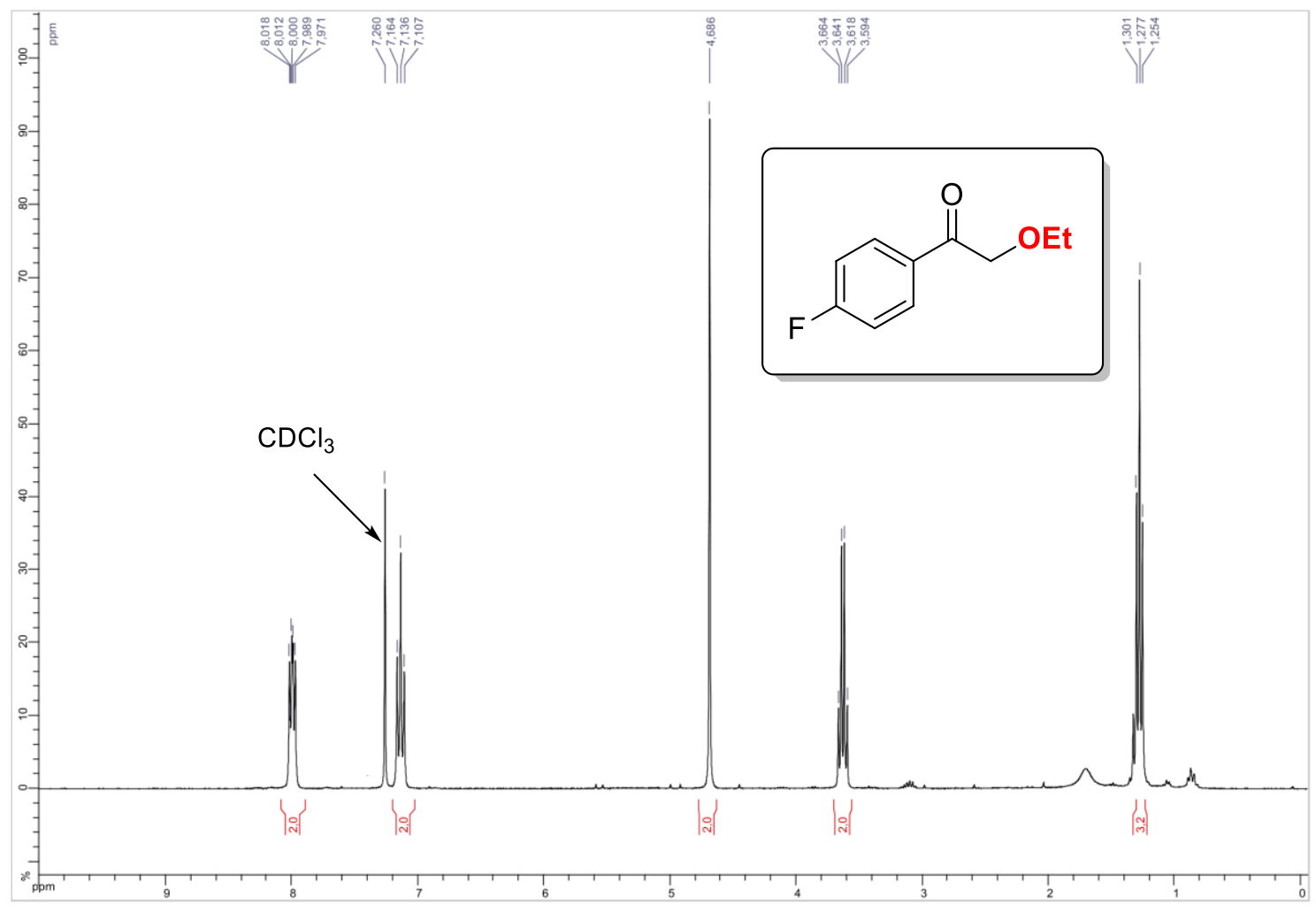

${ }^{13} \mathrm{C}$ NMR (75 MHz, CDCl3)

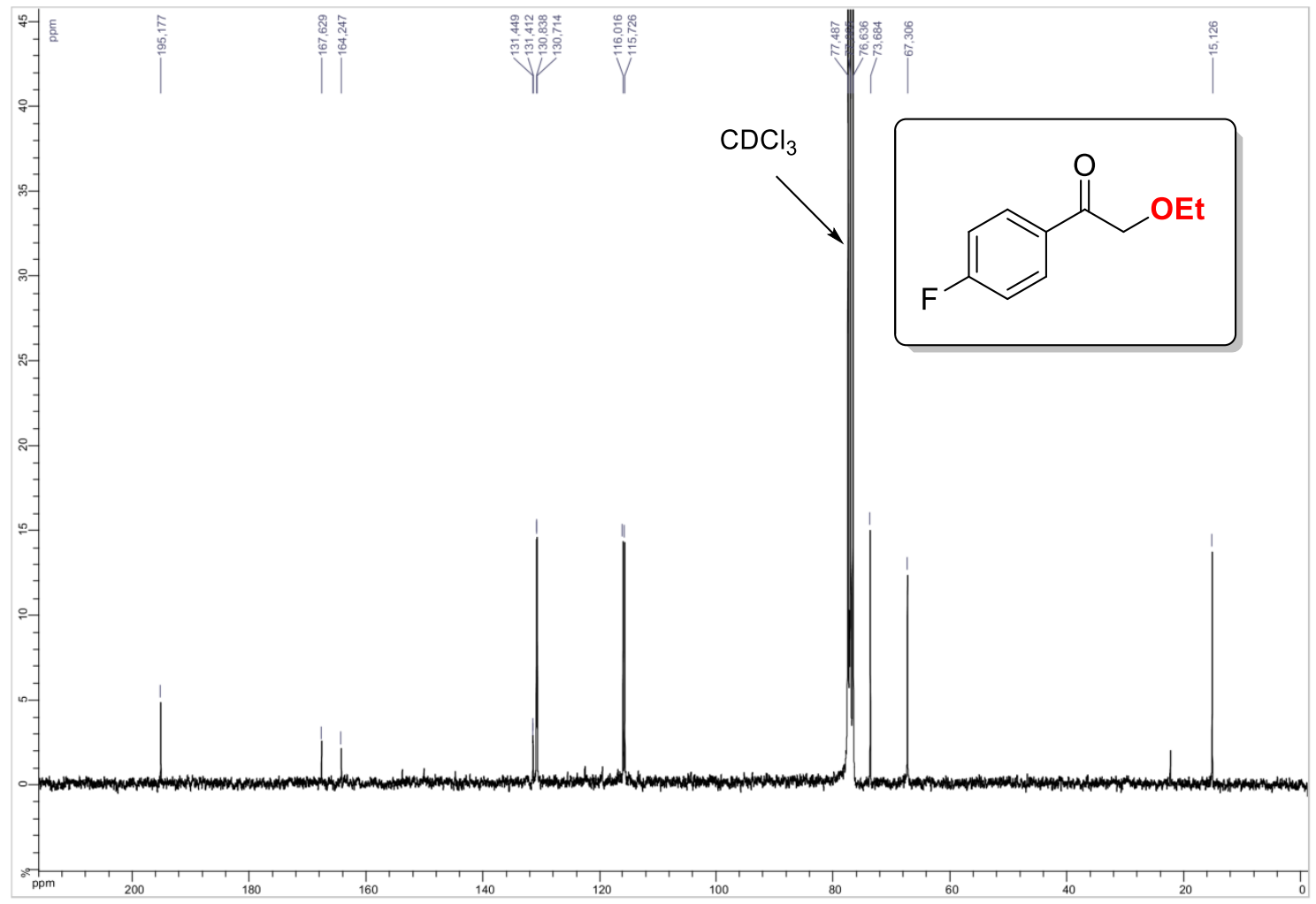


${ }^{19}$ F NMR (282 MHz, CDCl3)

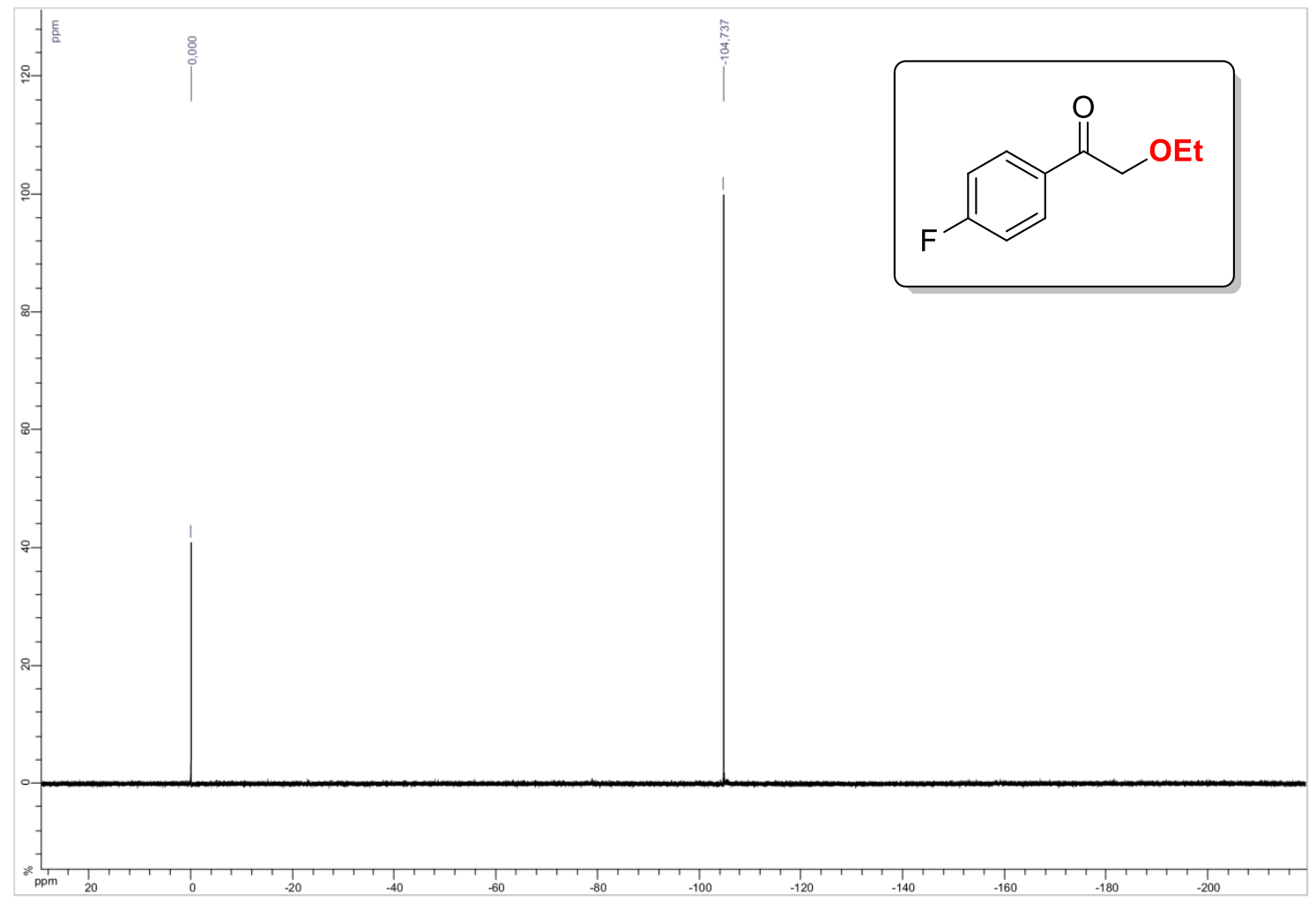


3-(2-(4-Fluorophenyl)-2-oxoethoxy)propanenitrile 4ag

${ }^{1}$ H NMR (300 MHz, CDCl 3 )

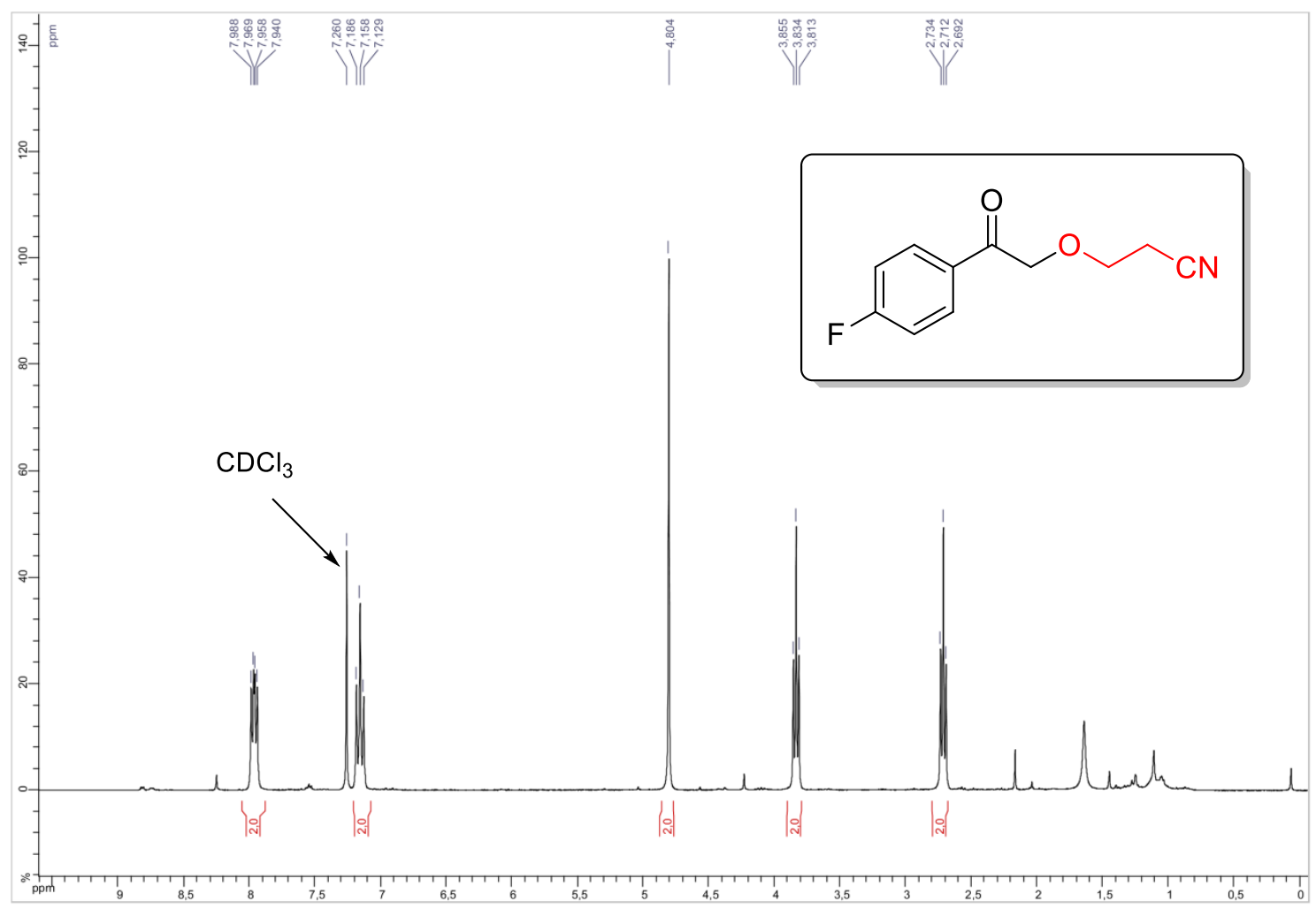

${ }^{13} \mathrm{C}$ NMR (75 MHz, CDCl3)

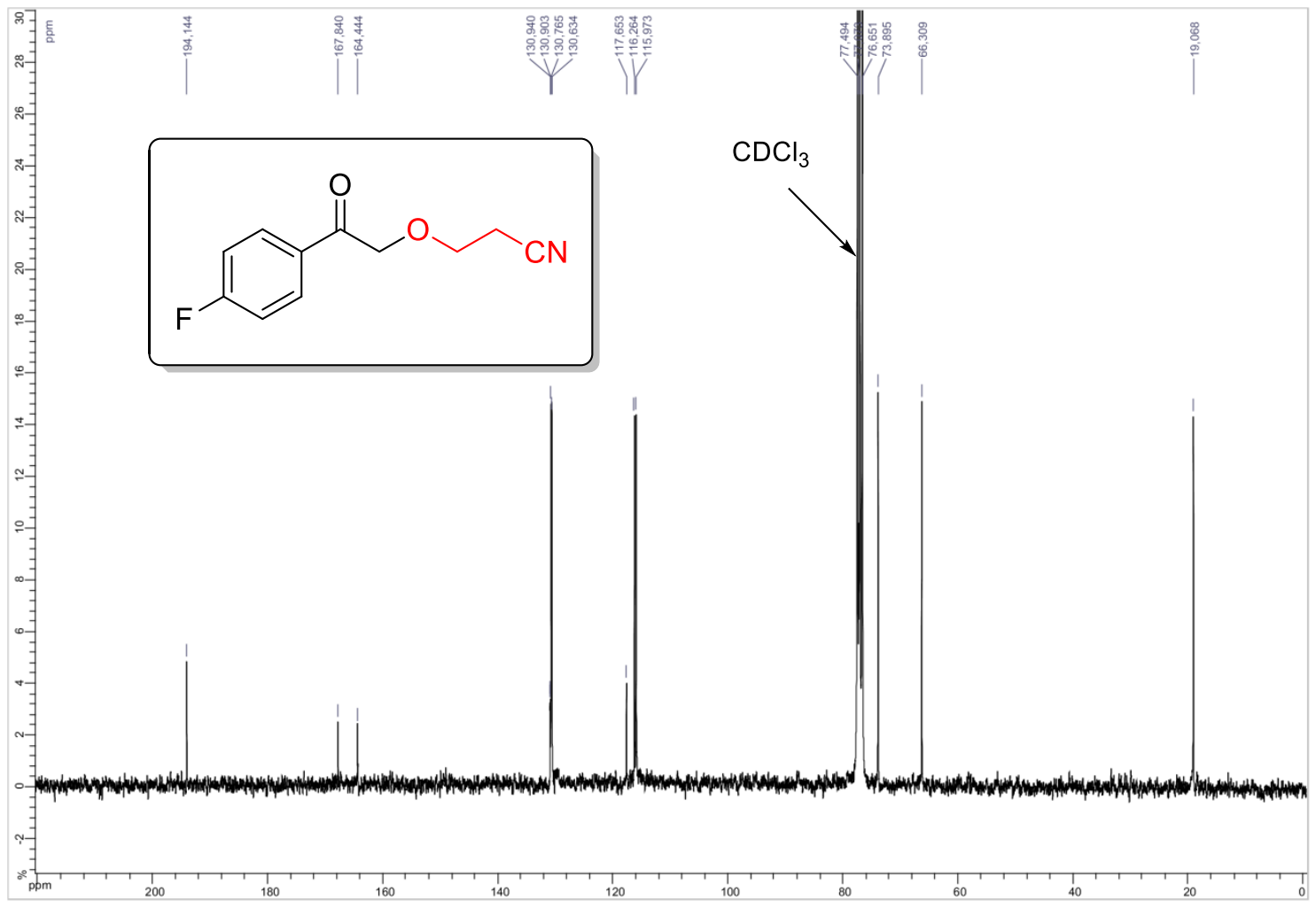


${ }^{19}$ F NMR (282 MHz, CDCl 3$)$

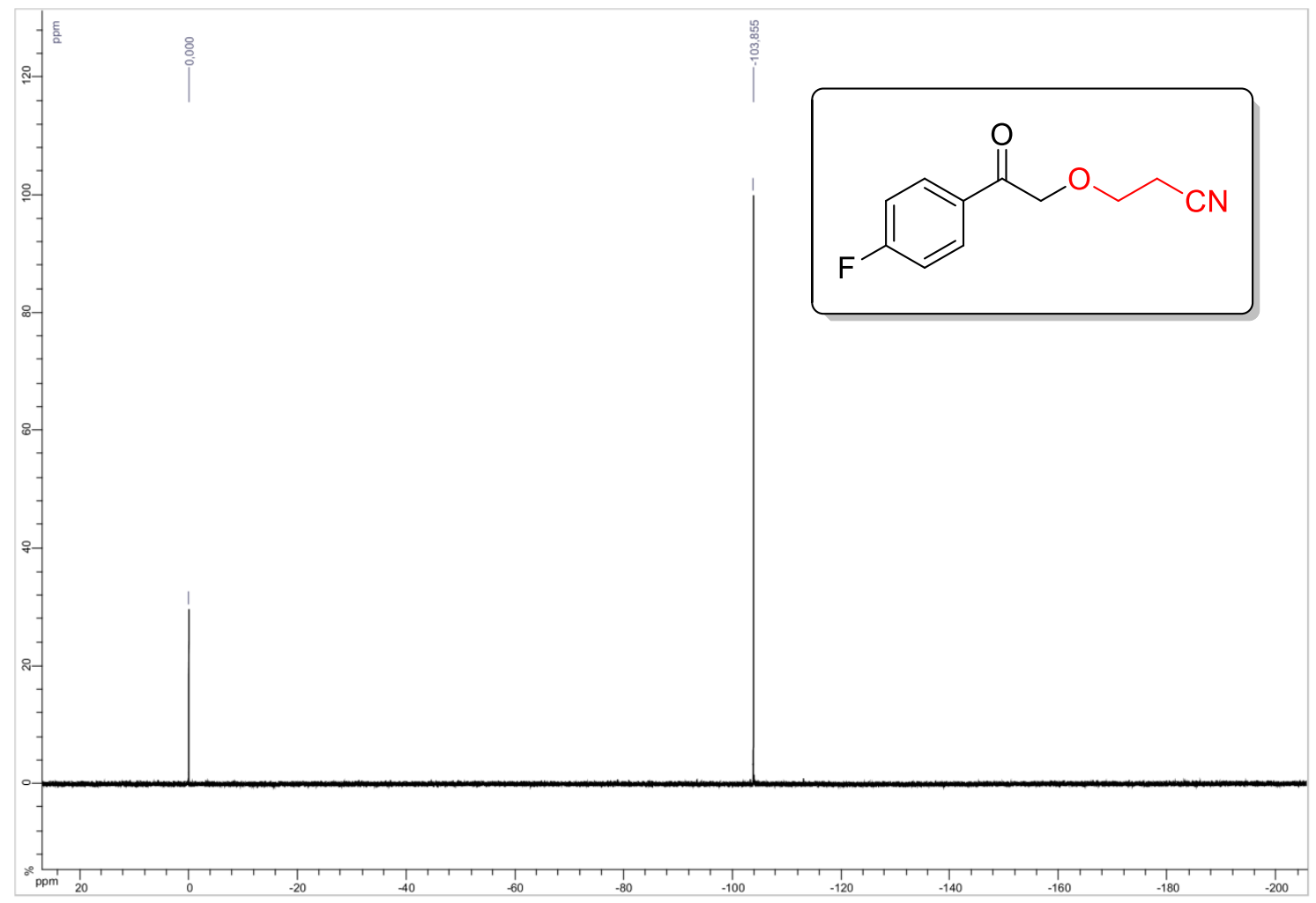


2-(But-2-yn-1-yloxy)-1-(4-fluorophenyl)ethanone 4ah

${ }^{1} \mathrm{H}$ NMR (300 MHz, CDCl 3$)$

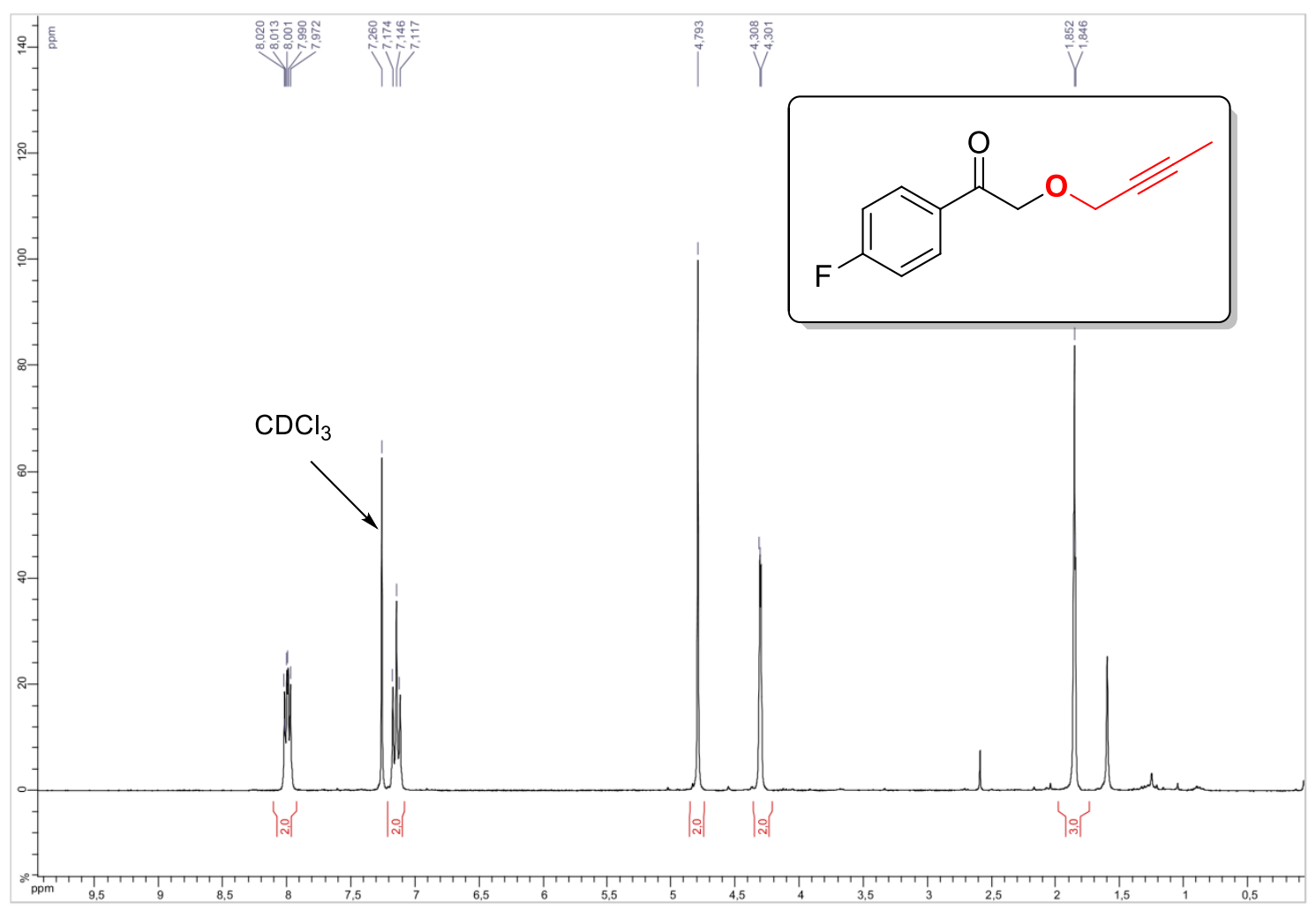

${ }^{13} \mathrm{C}$ NMR (75 MHz, CDCl3)

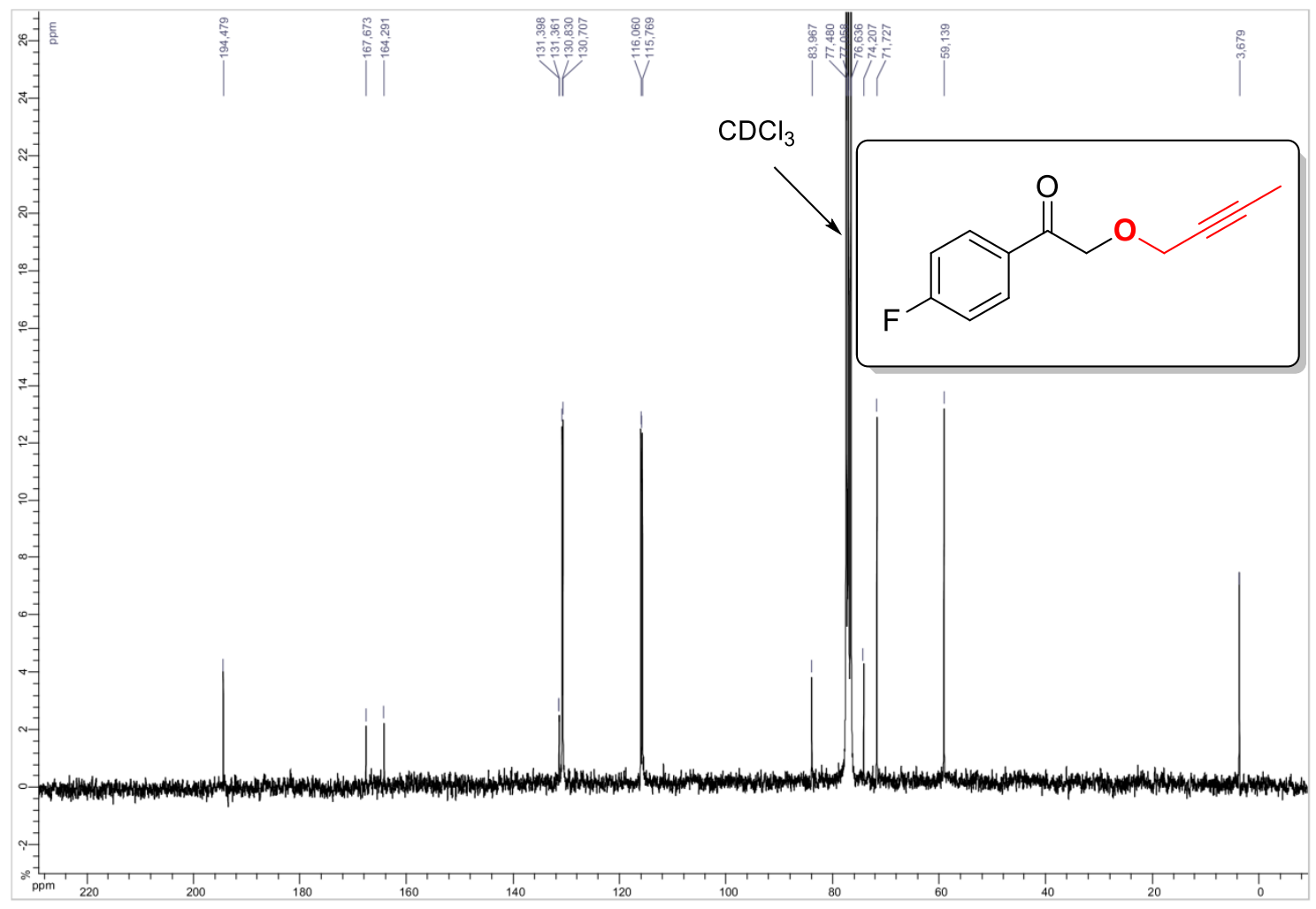


${ }^{19}$ F NMR (282 MHz, CDCl3)

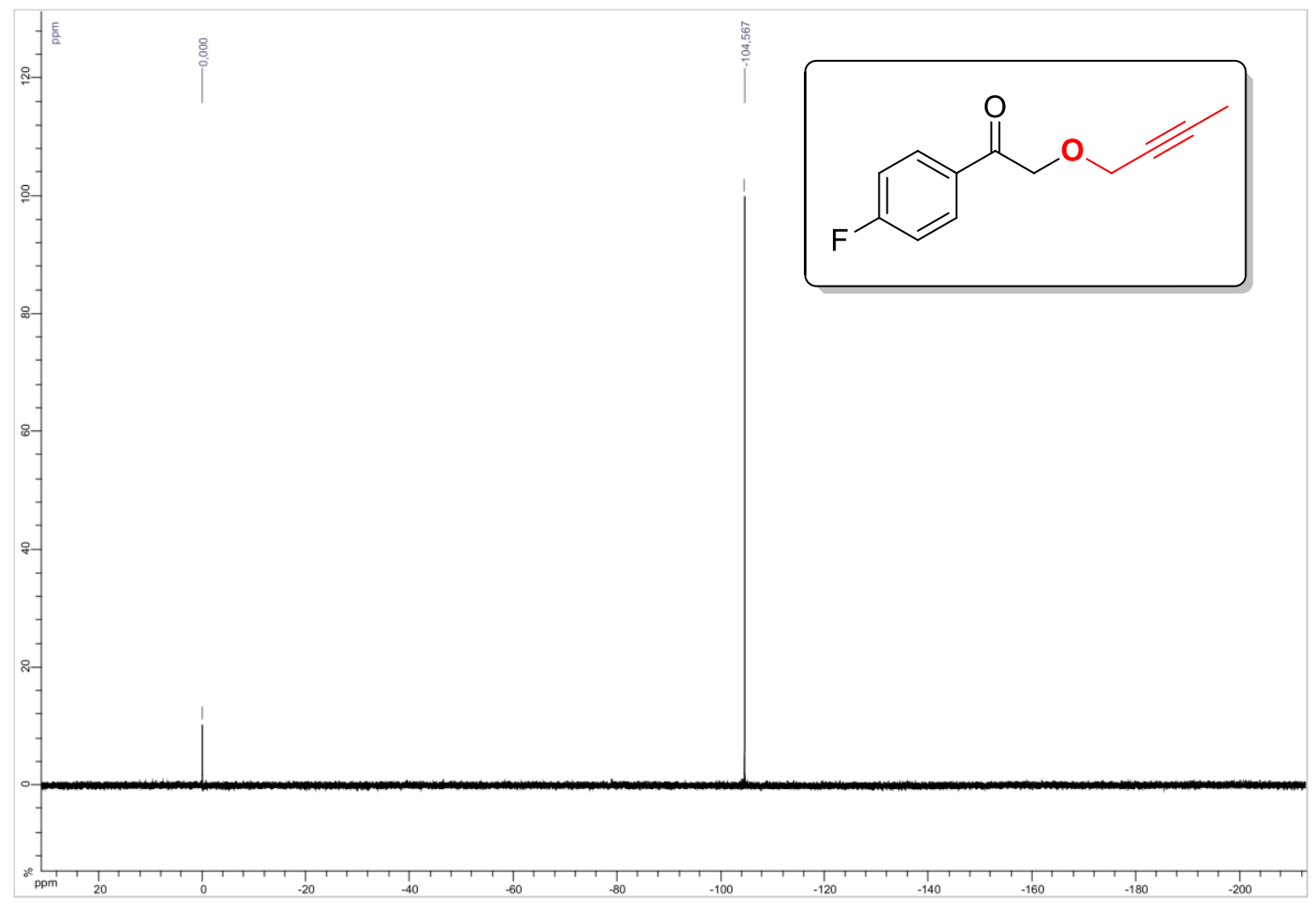


1-(4-Fluorophenyl)-2-(2-phenoxyethoxy)ethanone 4ai

${ }^{1} \mathrm{H}$ NMR (300 MHz, CDCl 3 )

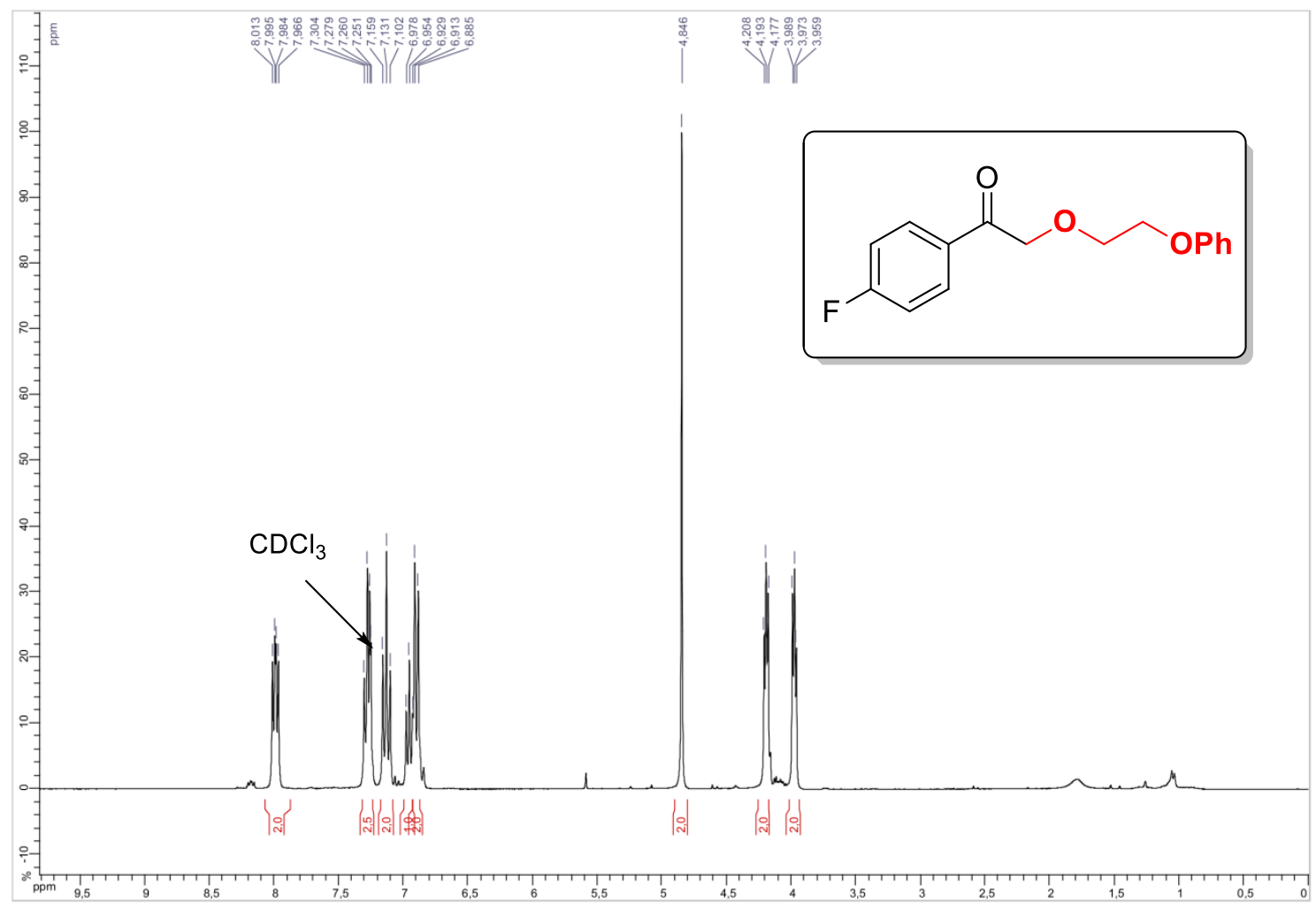

${ }^{13} \mathrm{C}$ NMR (75 MHz, CDCl3)

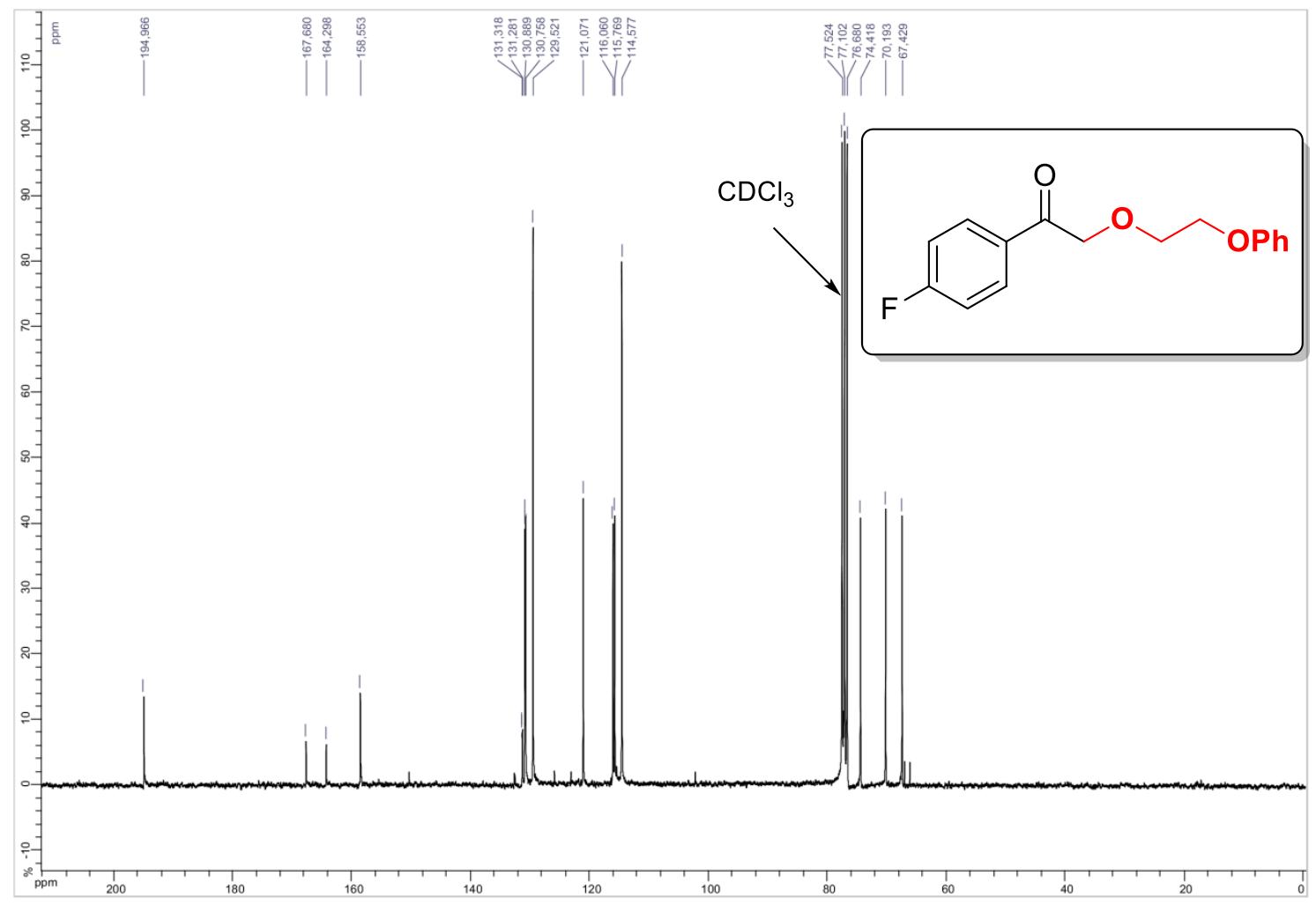


Methyl 3-(2-(3-phenylpropoxy)acetyl)benzoate 4aj

${ }^{1} \mathrm{H}$ NMR (300 MHz, $\left.\mathrm{CDCl}_{3}\right)$

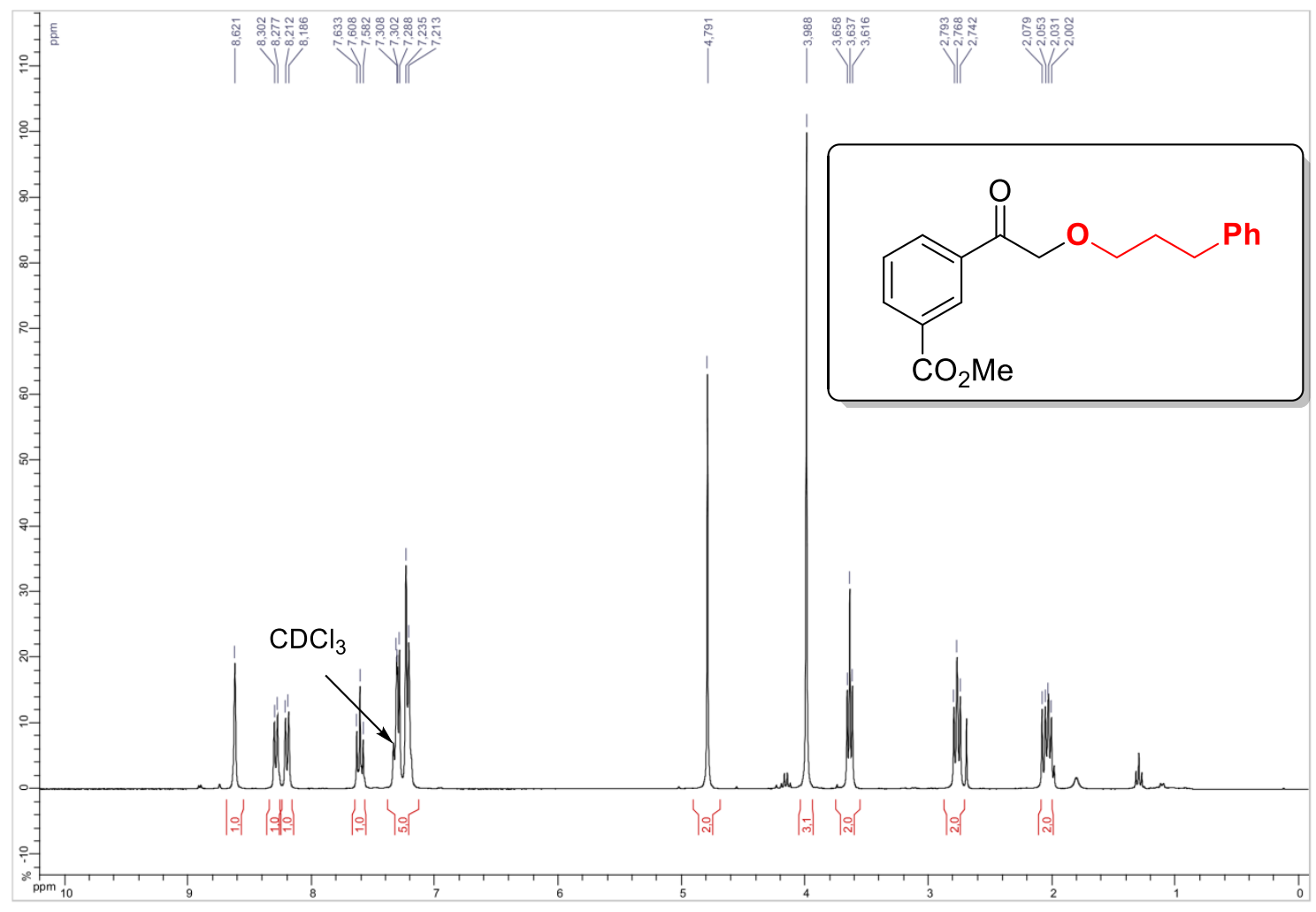

${ }^{13} \mathrm{C}$ NMR (75 MHz, CDCl3)

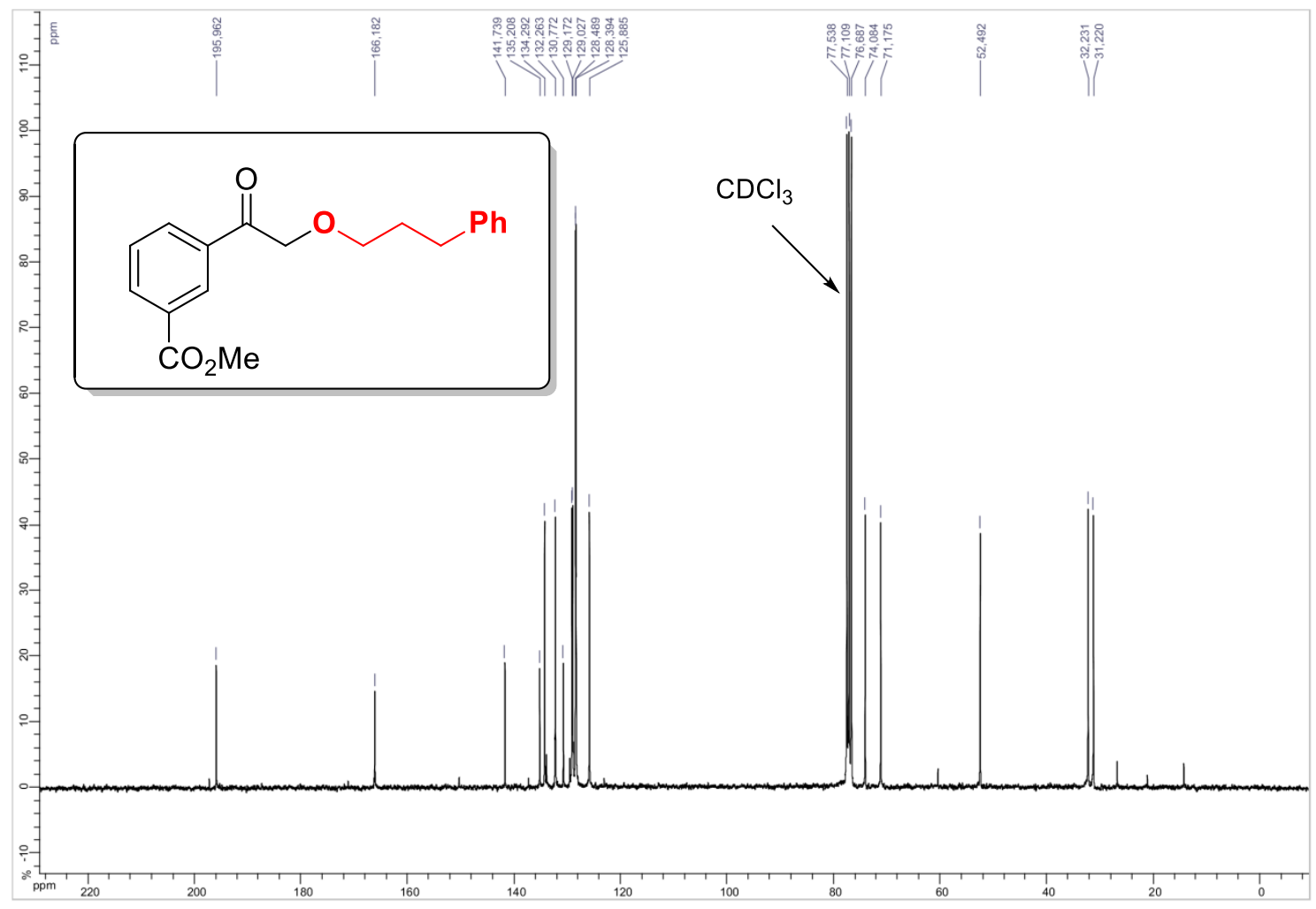


Methyl 3-(2-(3-(benzoyloxy)propoxy)acetyl)benzoate 4ak

${ }^{1}$ H NMR (300 MHz, CDCl 3$)$

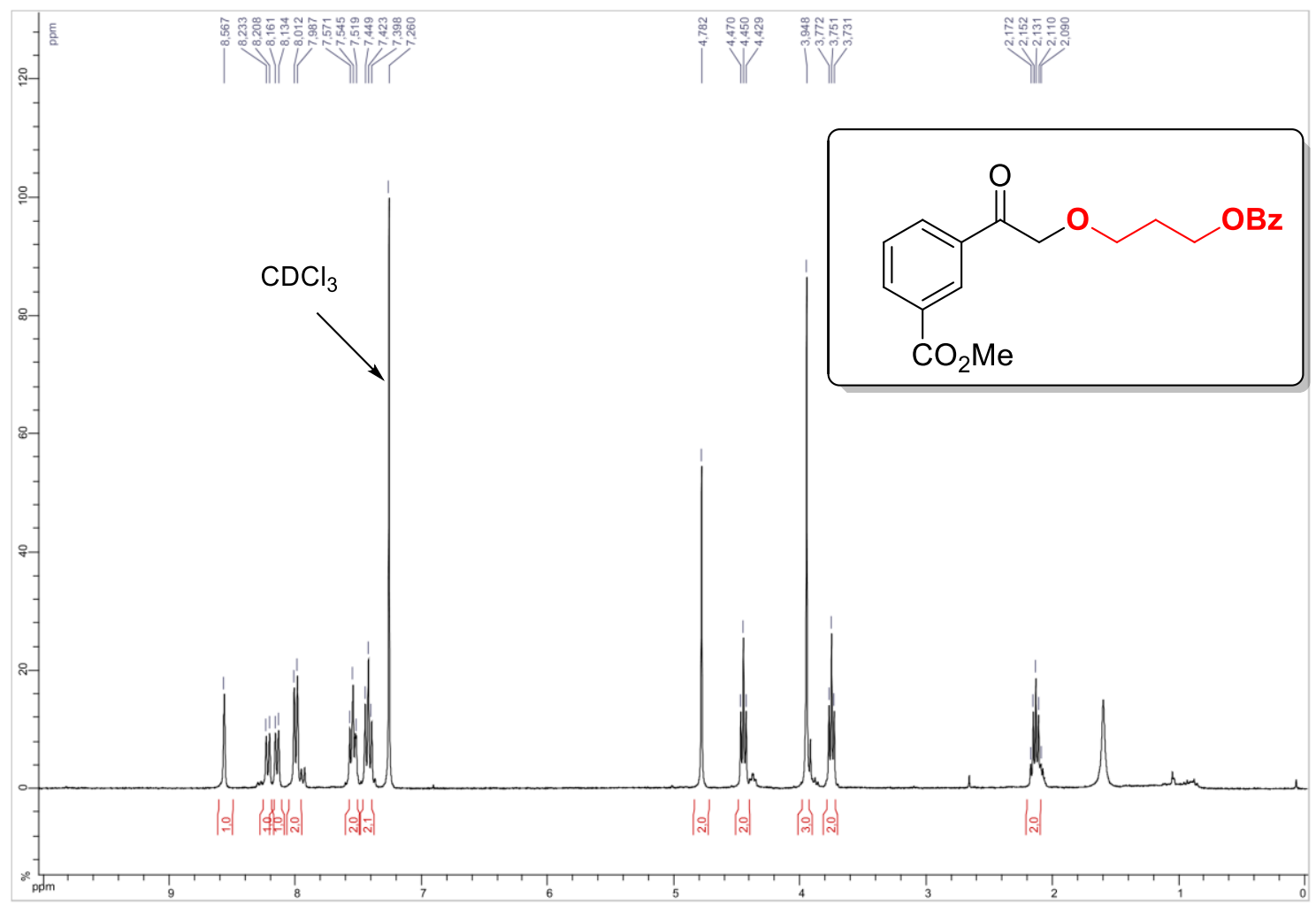

${ }^{13} \mathrm{C}$ NMR (75 MHz, CDCl3)

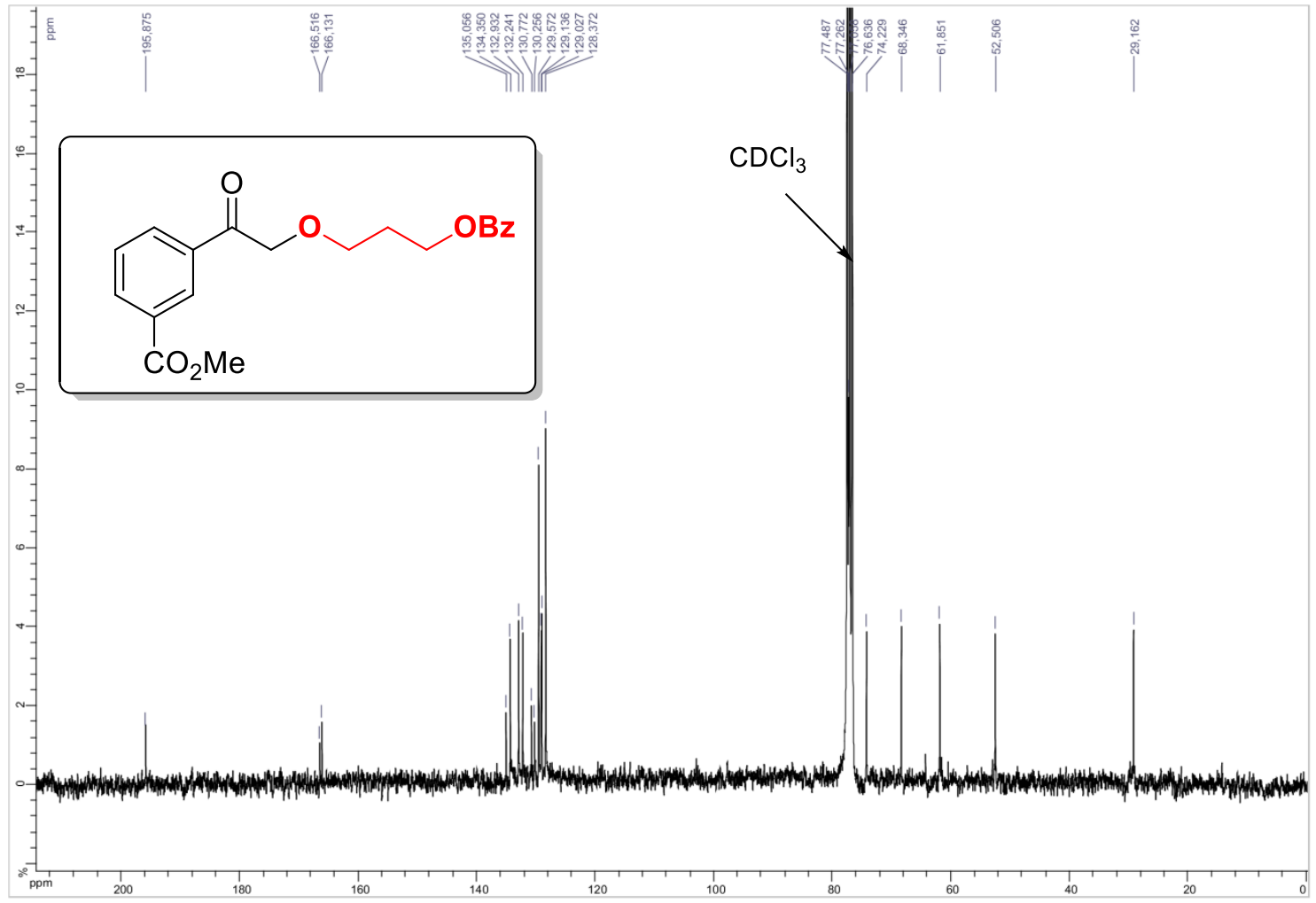


Methyl 3-(2-(3-(phenylsulfonyl)propoxy)acetyl)benzoate 4al ${ }^{1} \mathrm{H}$ NMR (300 MHz, $\left.\mathrm{CDCl}_{3}\right)$

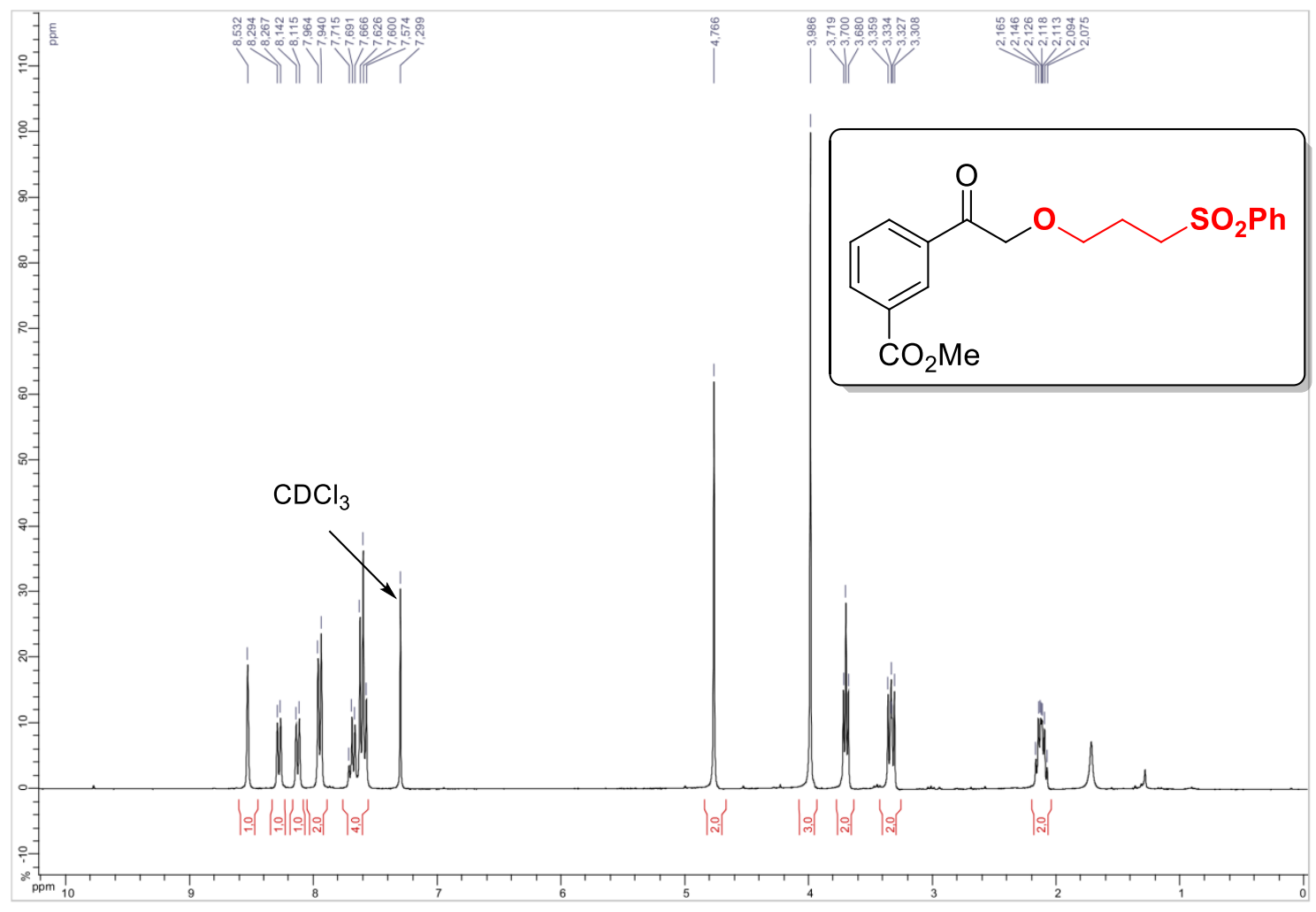

${ }^{13} \mathrm{C}$ NMR (75 MHz, CDCl3)

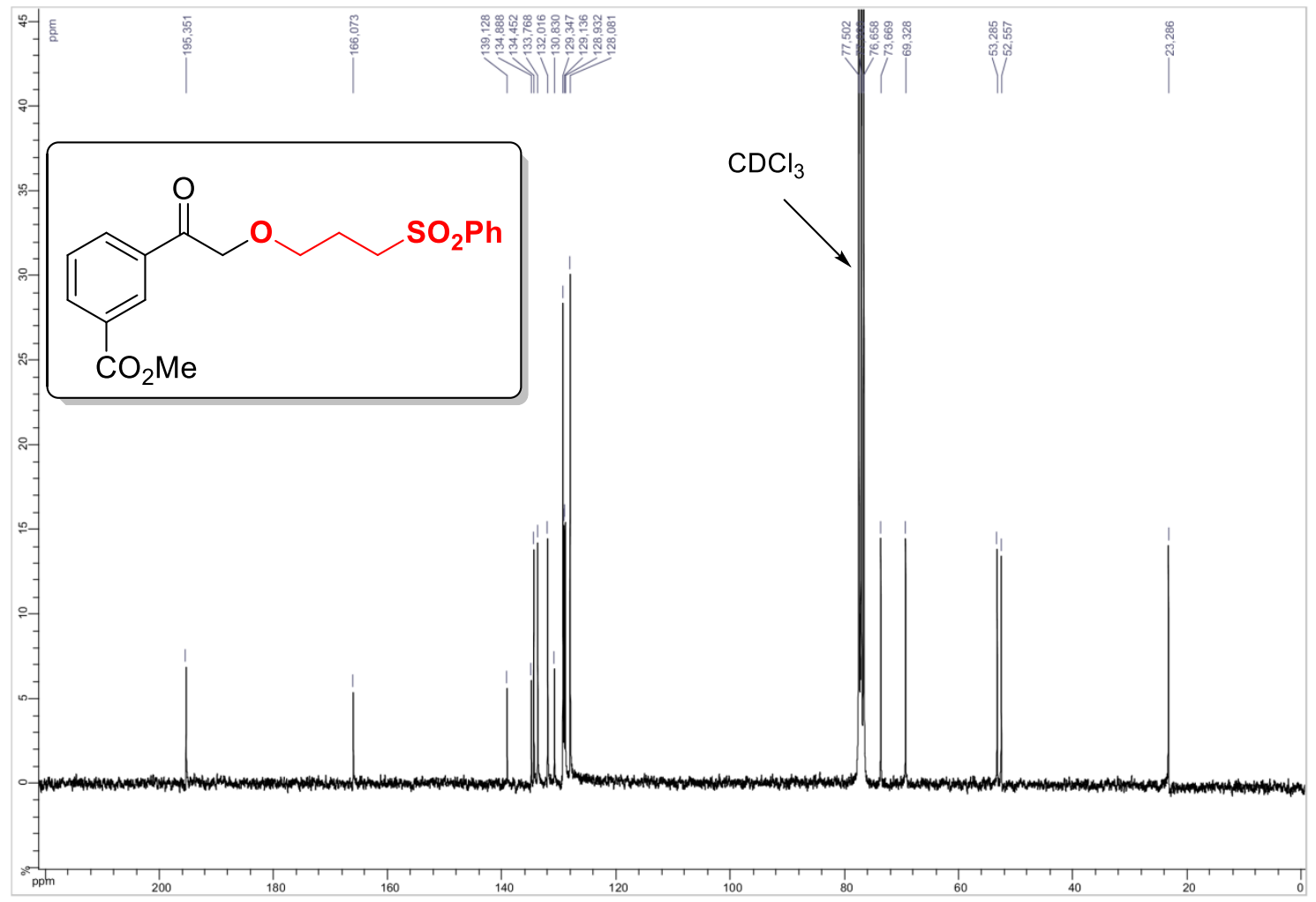


1-(4-Fluorophenyl)-2-isopropoxyethanone 4am

${ }^{1} \mathrm{H}$ NMR (300 MHz, $\left.\mathrm{CDCl}_{3}\right)$

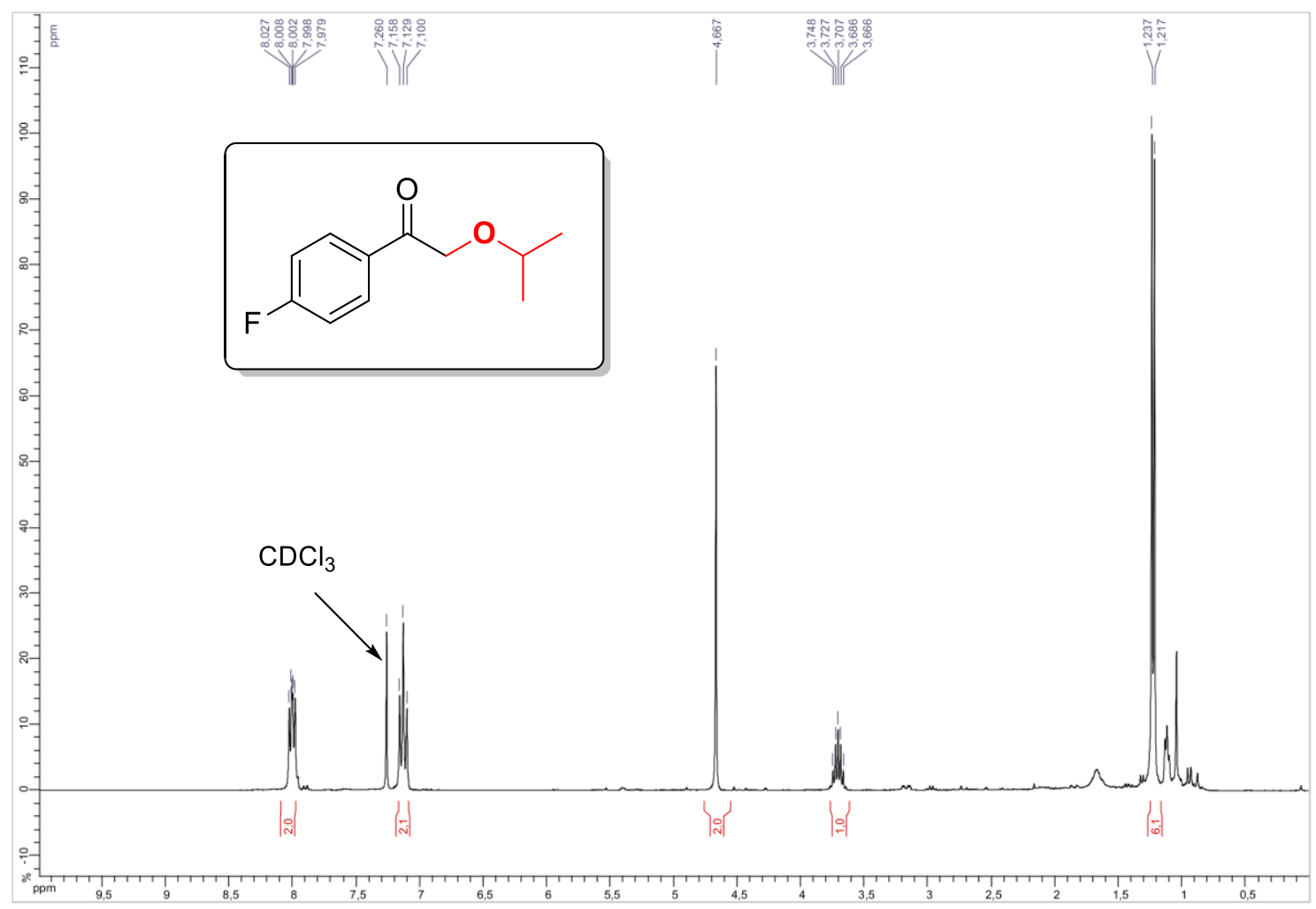

${ }^{13} \mathrm{C}$ NMR (75 MHz, CDCl3)

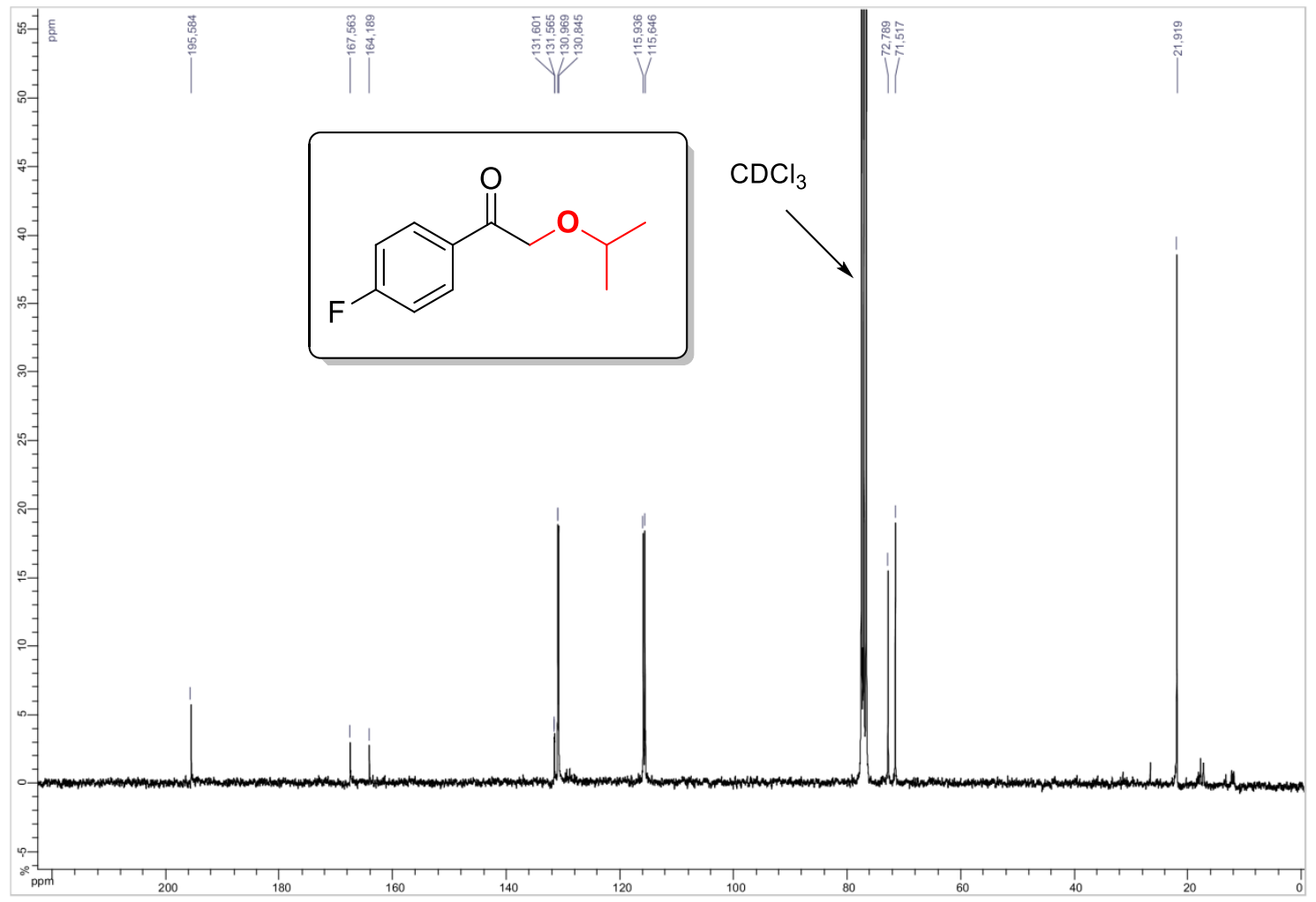


${ }^{19}$ F NMR (282 MHz, $\left.\mathrm{CDCl}_{3}\right)$

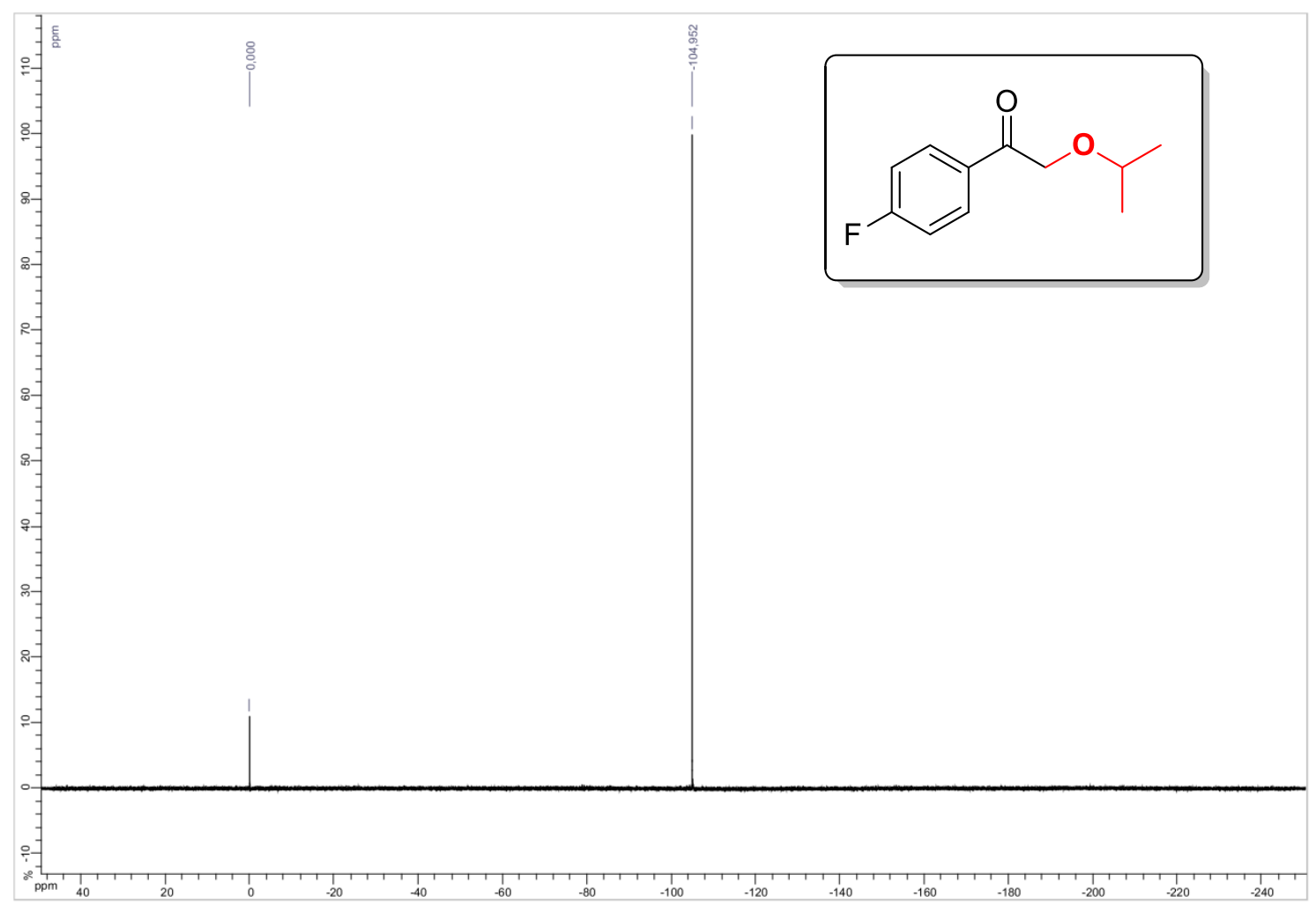


2-((1,3-Difluoropropan-2-yl)oxy)-1-(4-fluorophenyl)ethanone 4an ${ }^{1} \mathrm{H}$ NMR (300 MHz, CDCl 3$)$

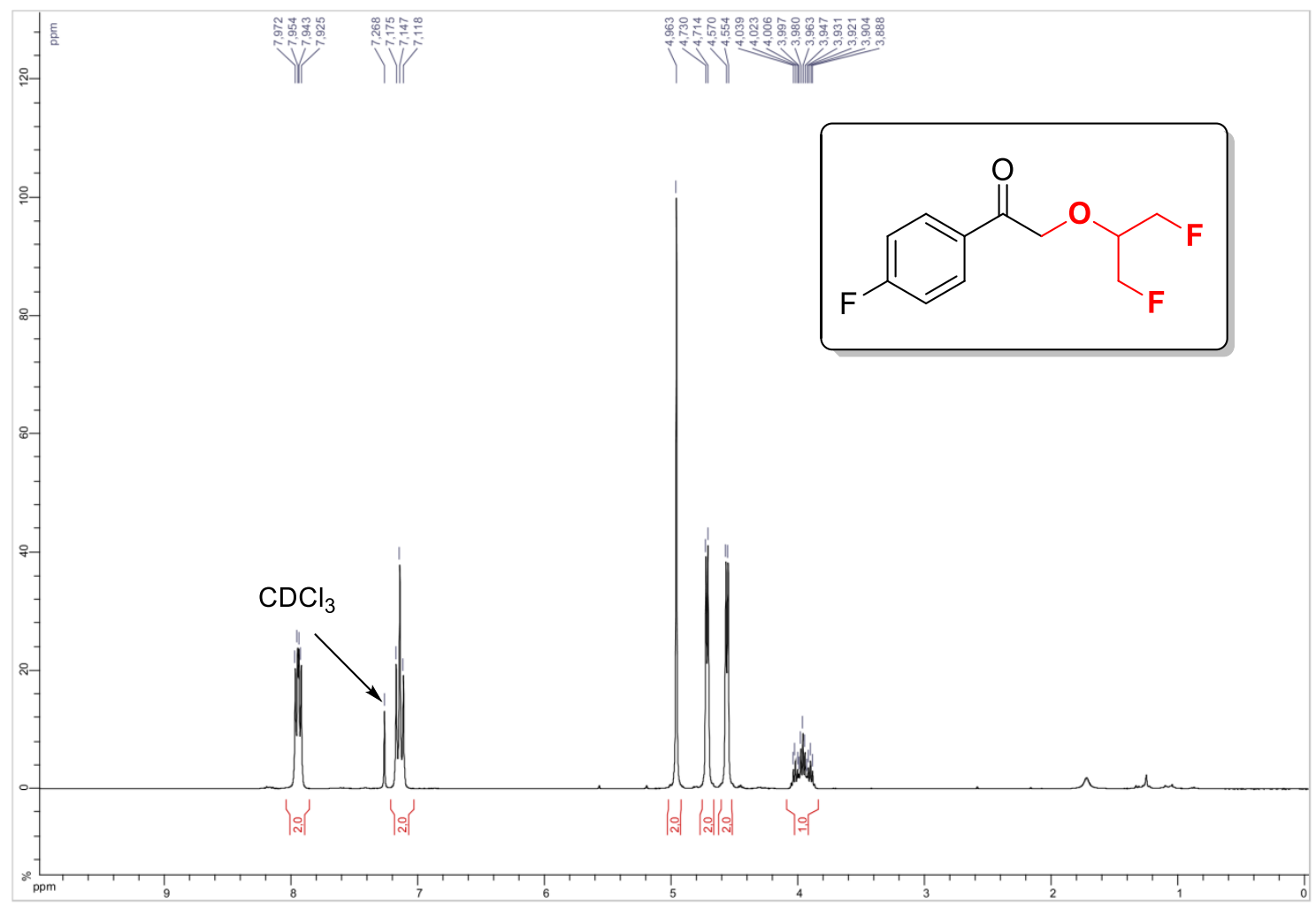

${ }^{13} \mathrm{C}$ NMR (75 MHz, CDCl 3$)$

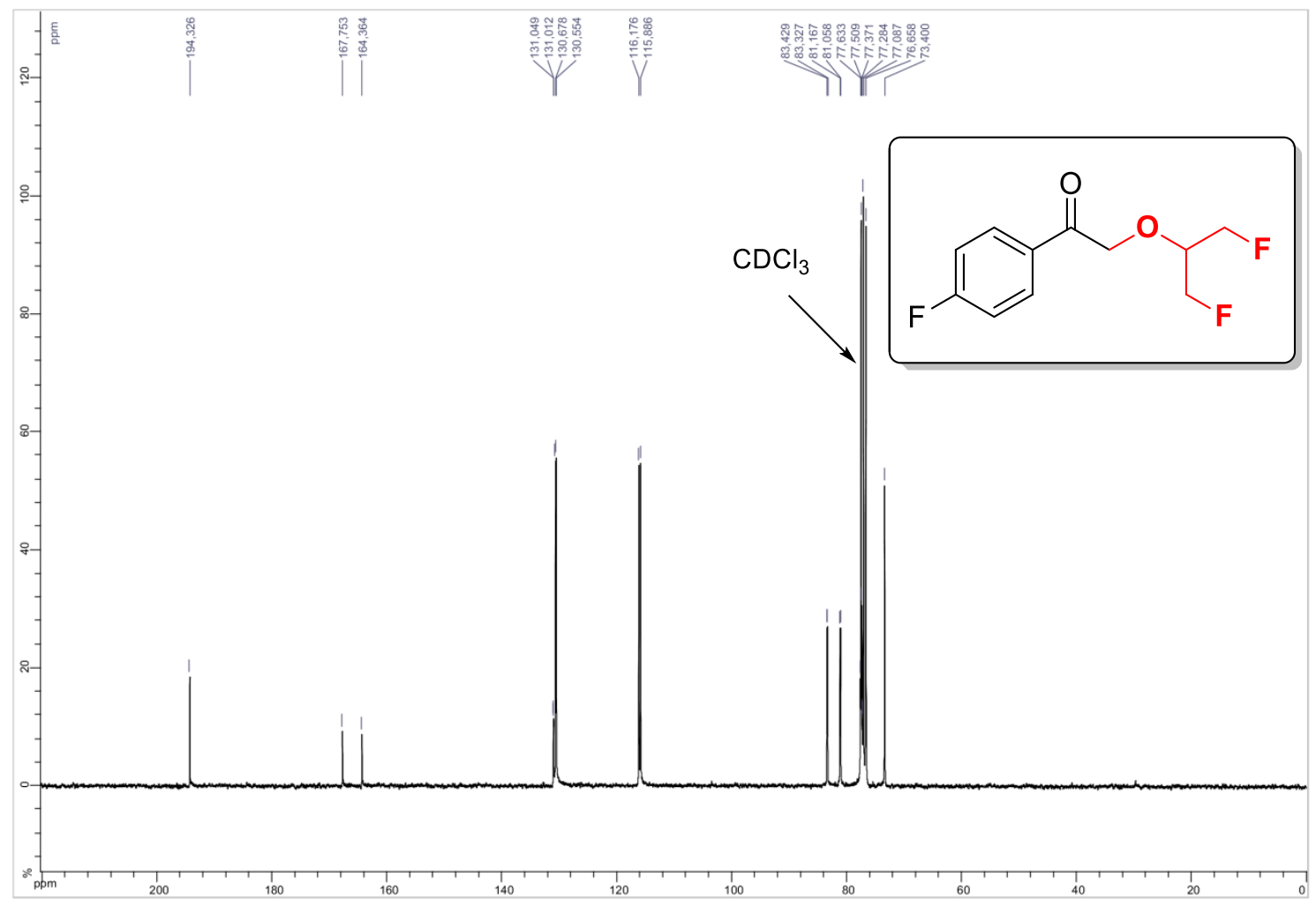


${ }^{19}$ F NMR (282 MHz, CDCl3)

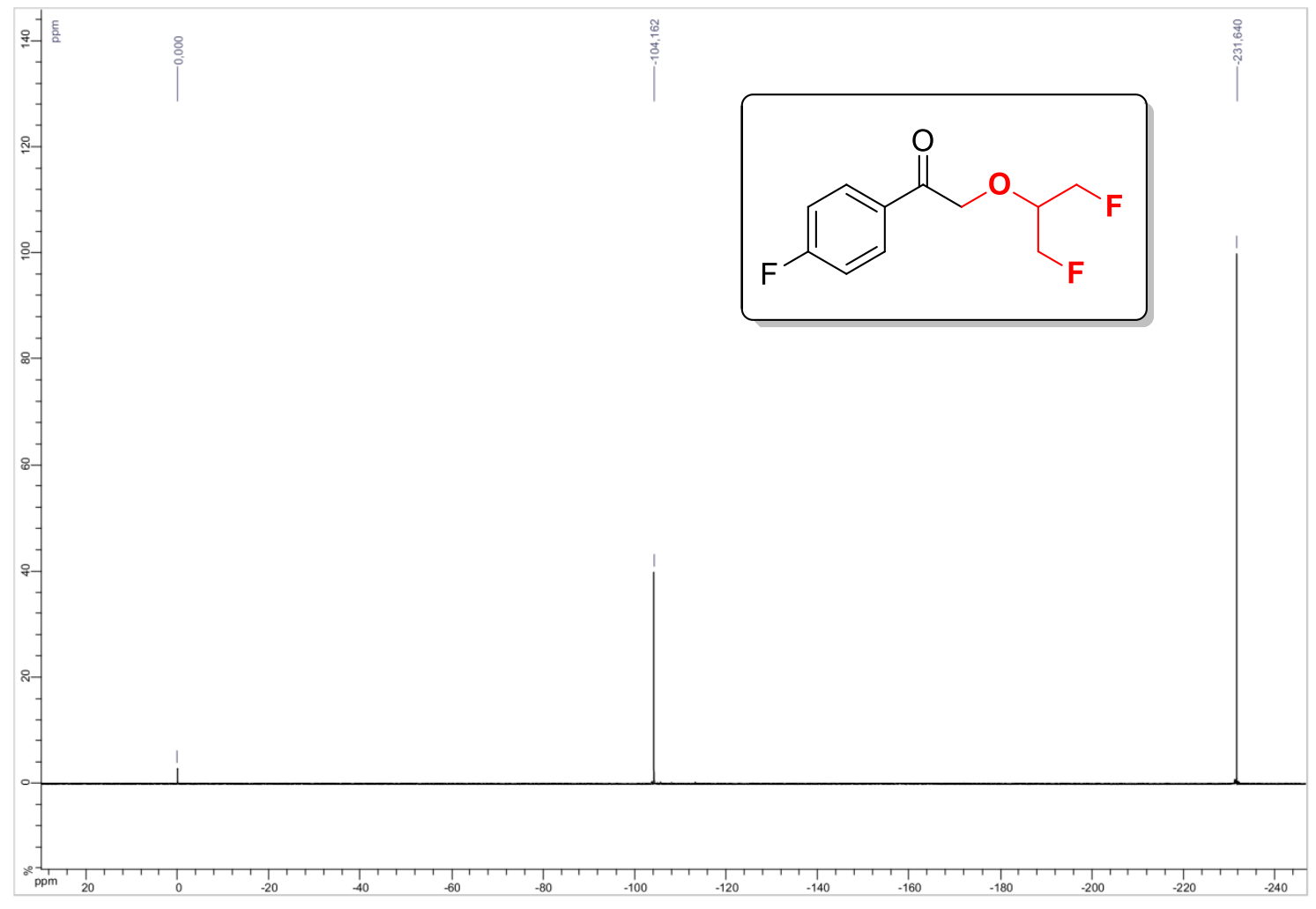


3-(2-Methoxyhexanoyl)oxazolidin-2-one 7a

${ }^{1} \mathrm{H}$ NMR (300 MHz, $\left.\mathrm{CDCl}_{3}\right)$

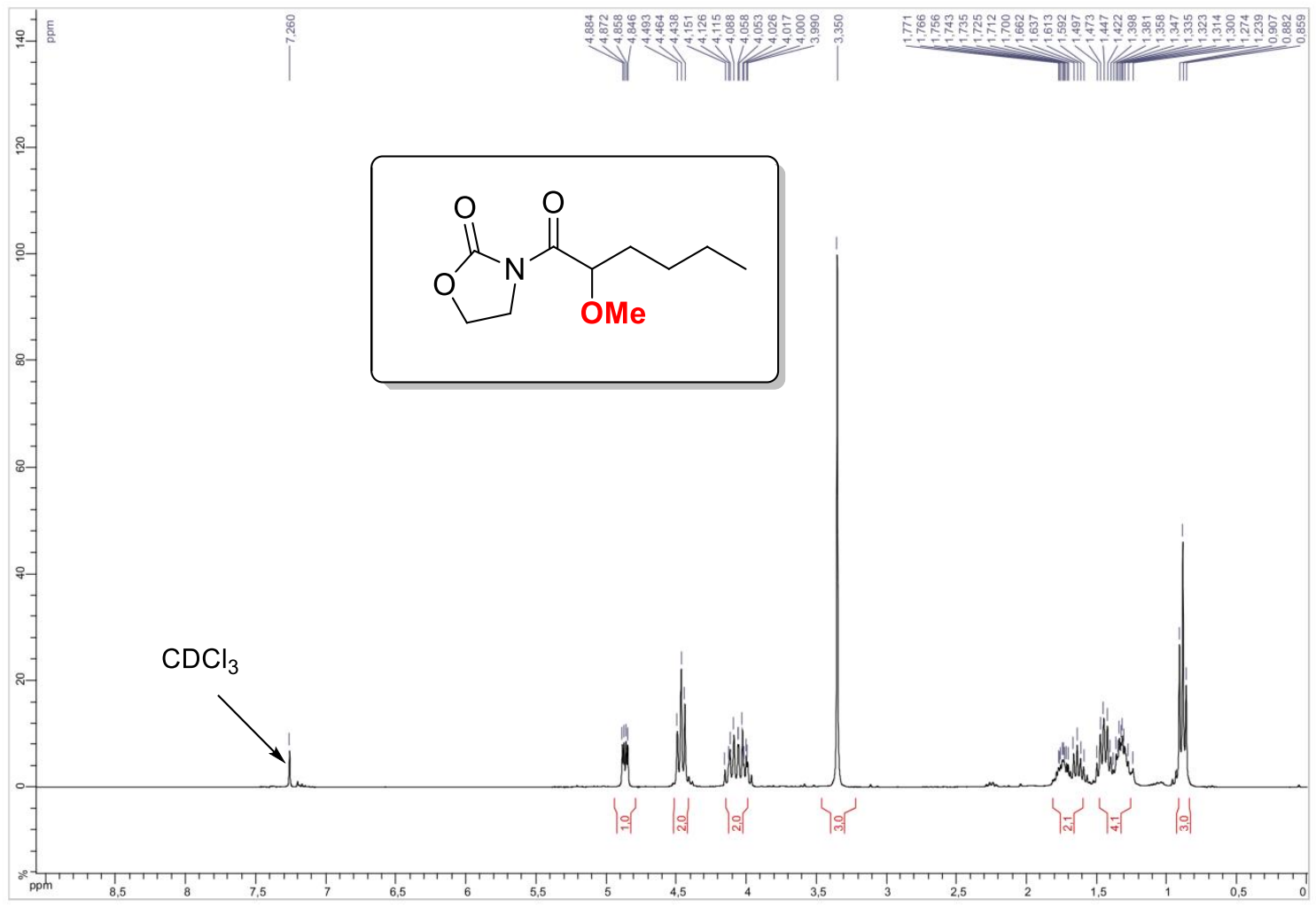

${ }^{13} \mathrm{C}$ NMR (75 MHz, CDCl3)

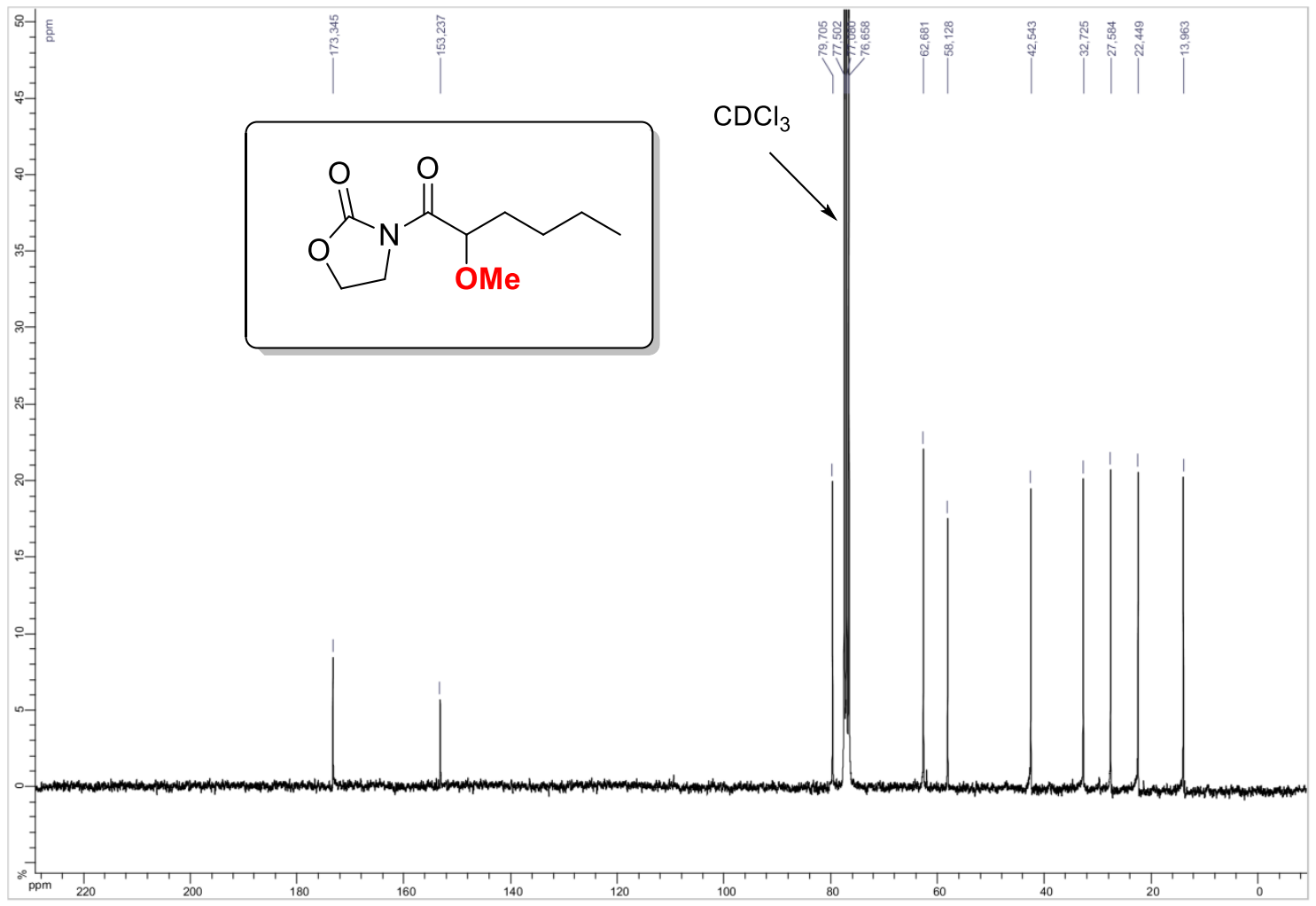


3-(6-bromo-2-methoxyhexanoyl)oxazolidin-2-one 7b

${ }^{1} \mathrm{H}$ NMR (300 MHz, CDCl 3$)$

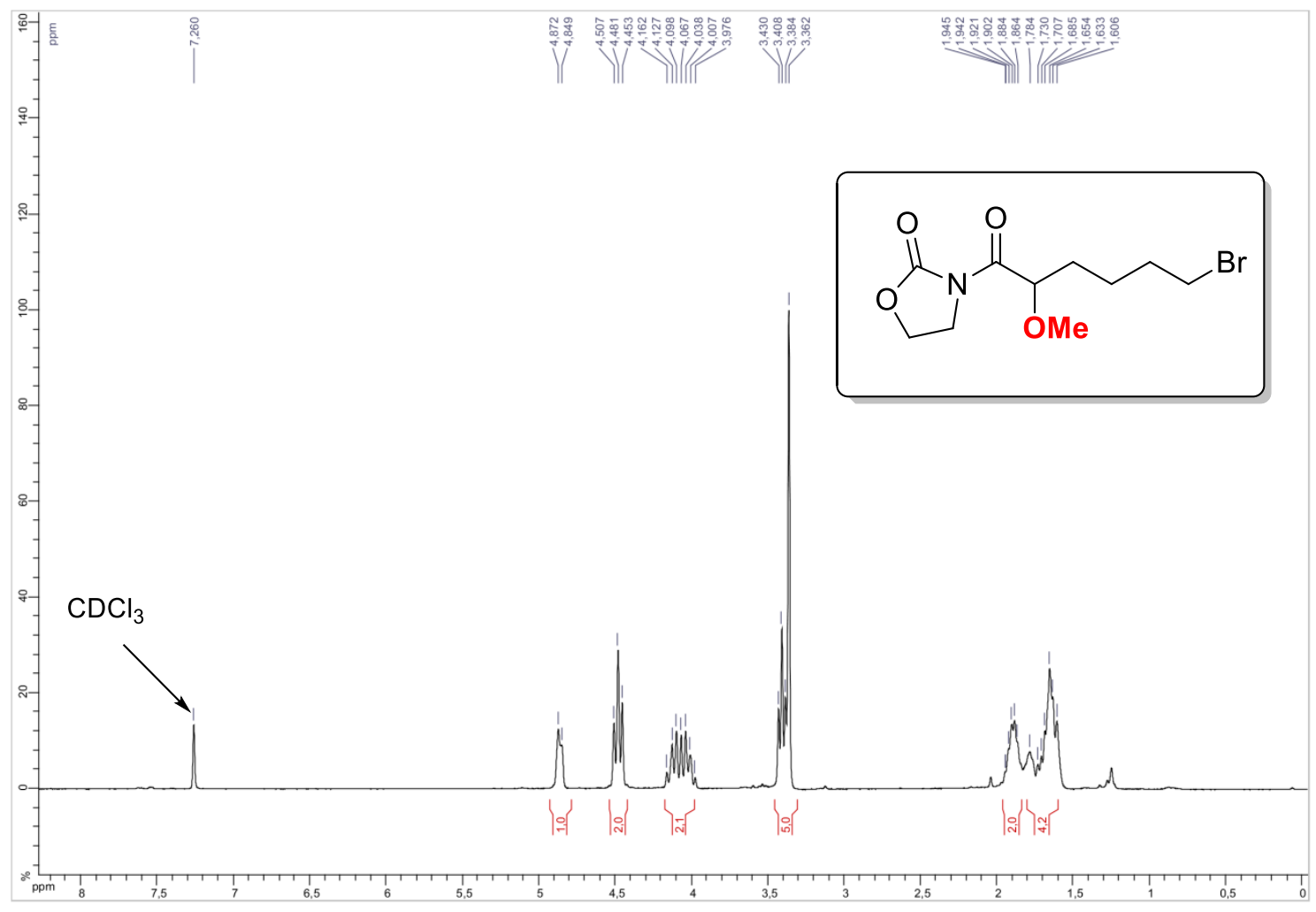

${ }^{13} \mathrm{C}$ NMR (75 MHz, CDCl3)

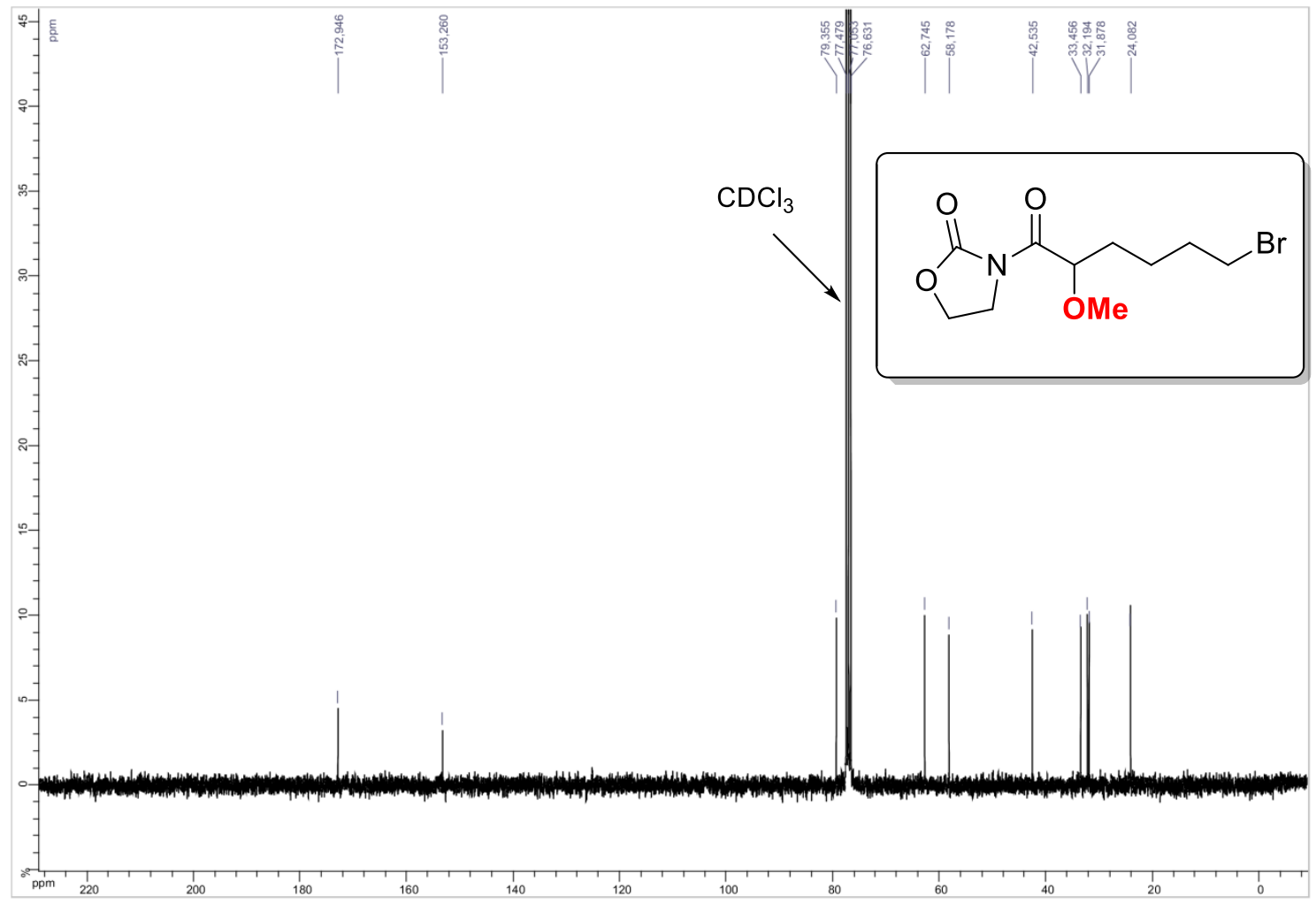


3-(2-methoxy-3-(methylthio)propanoyl)oxazolidin-2-one 7c

${ }^{1} \mathrm{H}$ NMR (300 MHz, CDCl 3$)$

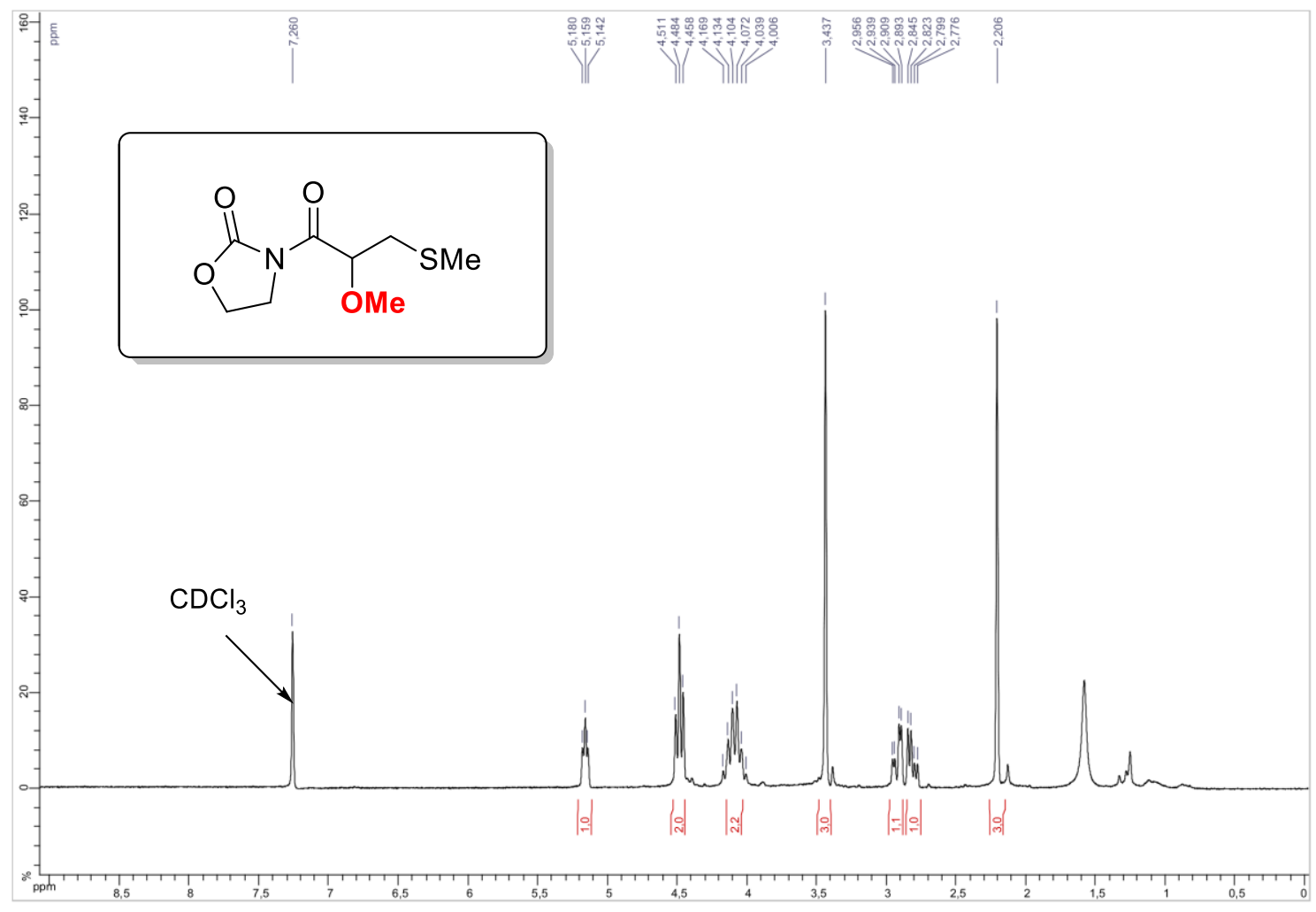

${ }^{13} \mathrm{C}$ NMR (75 MHz, CDCl3)

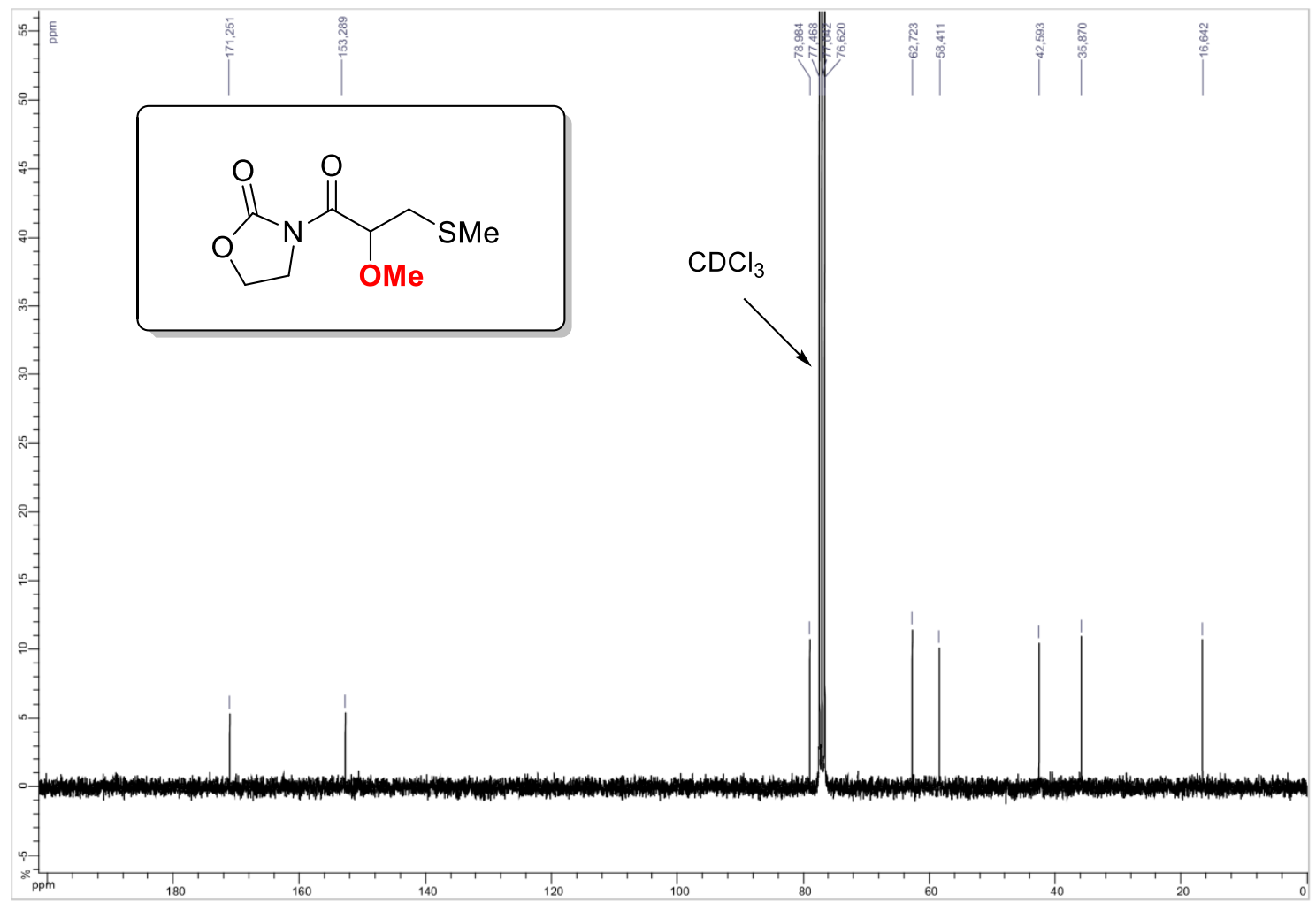


3-(4,4,4-trifluoro-2-methoxybutanoyl)oxazolidin-2-one 7d ${ }^{1} \mathrm{H}$ NMR (300 MHz, $\left.\mathrm{CDCl}_{3}\right)$

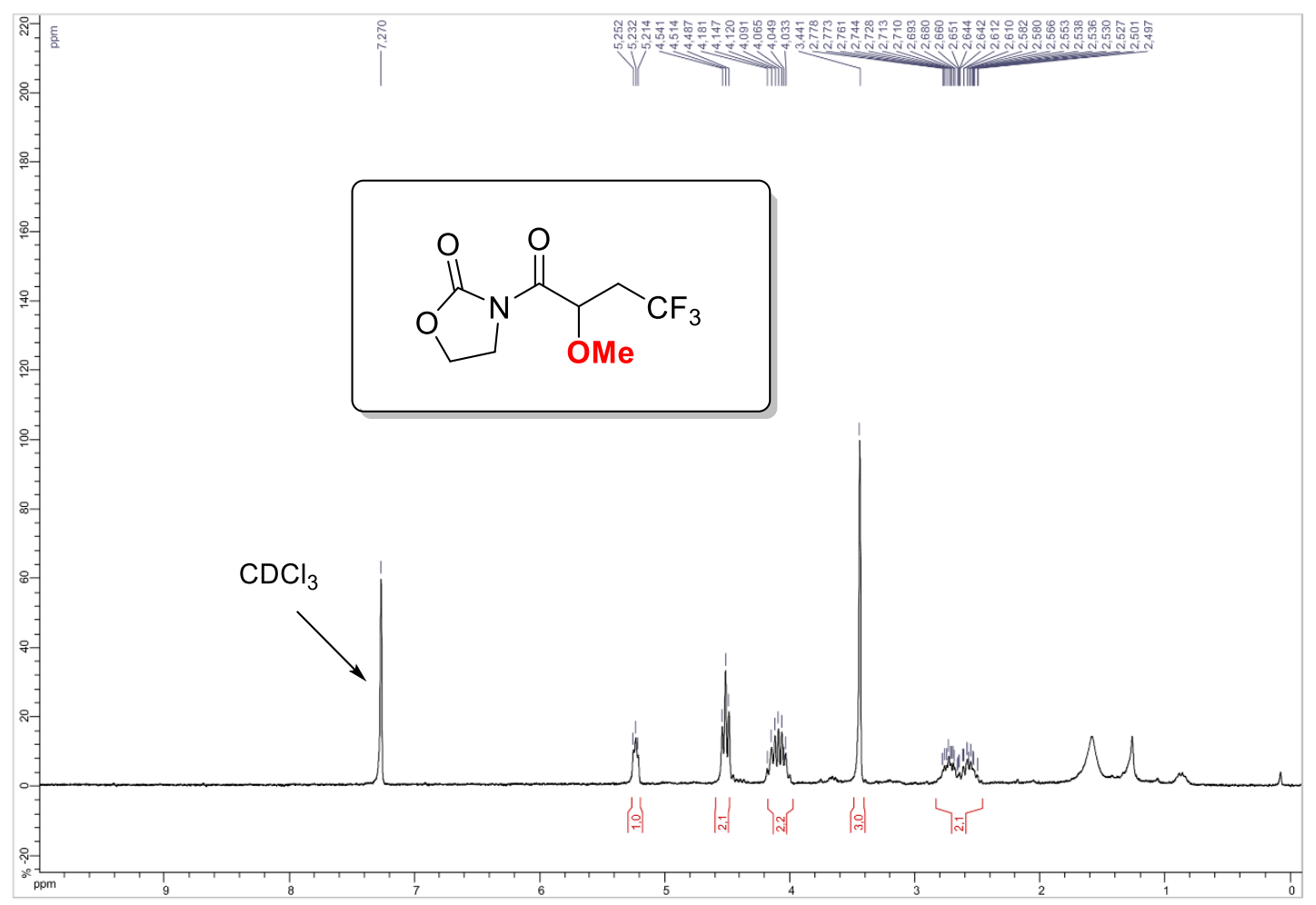

${ }^{13} \mathrm{C}$ NMR (75 MHz, CDCl3)

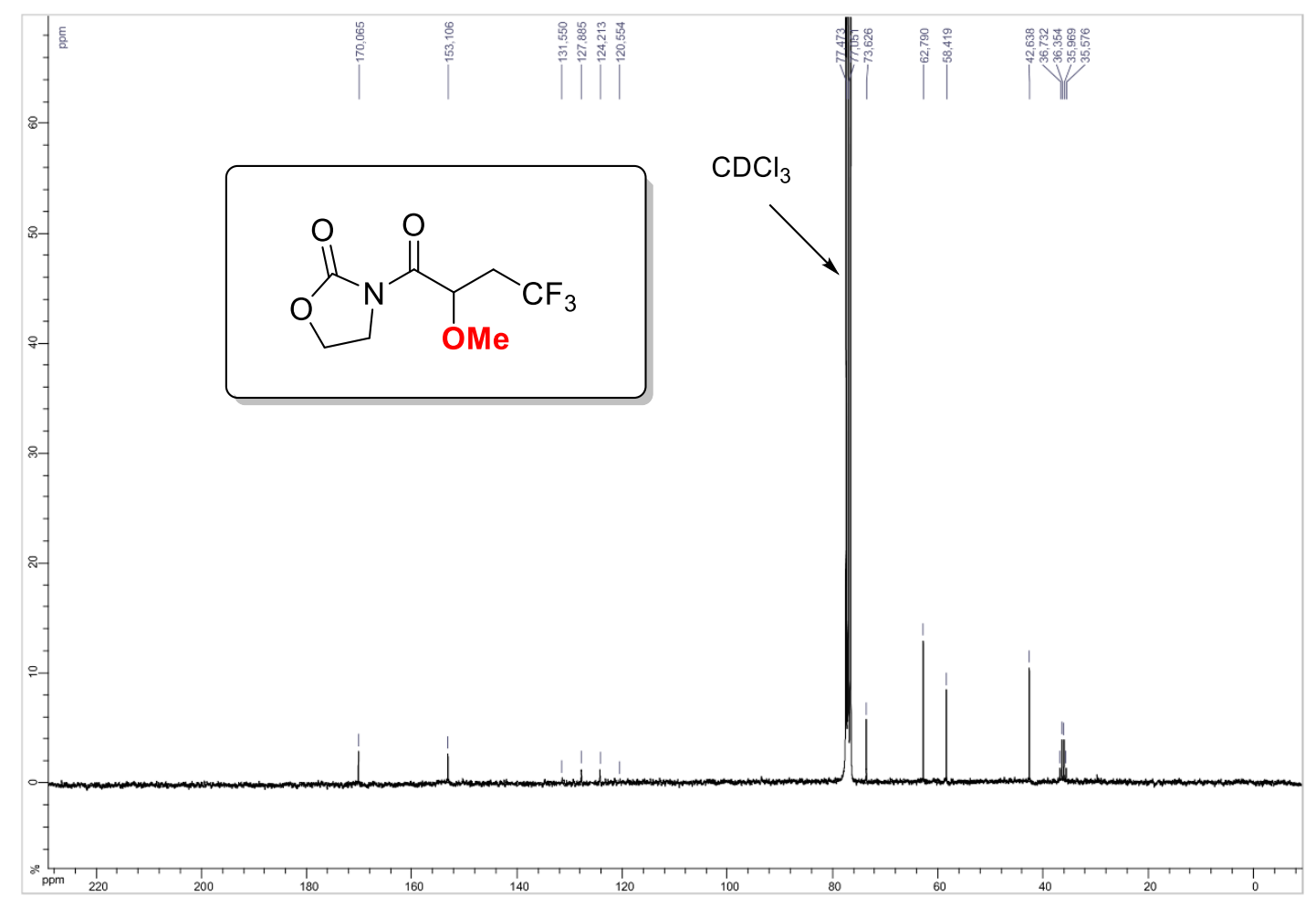


${ }^{19}$ F NMR (282 MHz, $\left.\mathrm{CDCl}_{3}\right)$

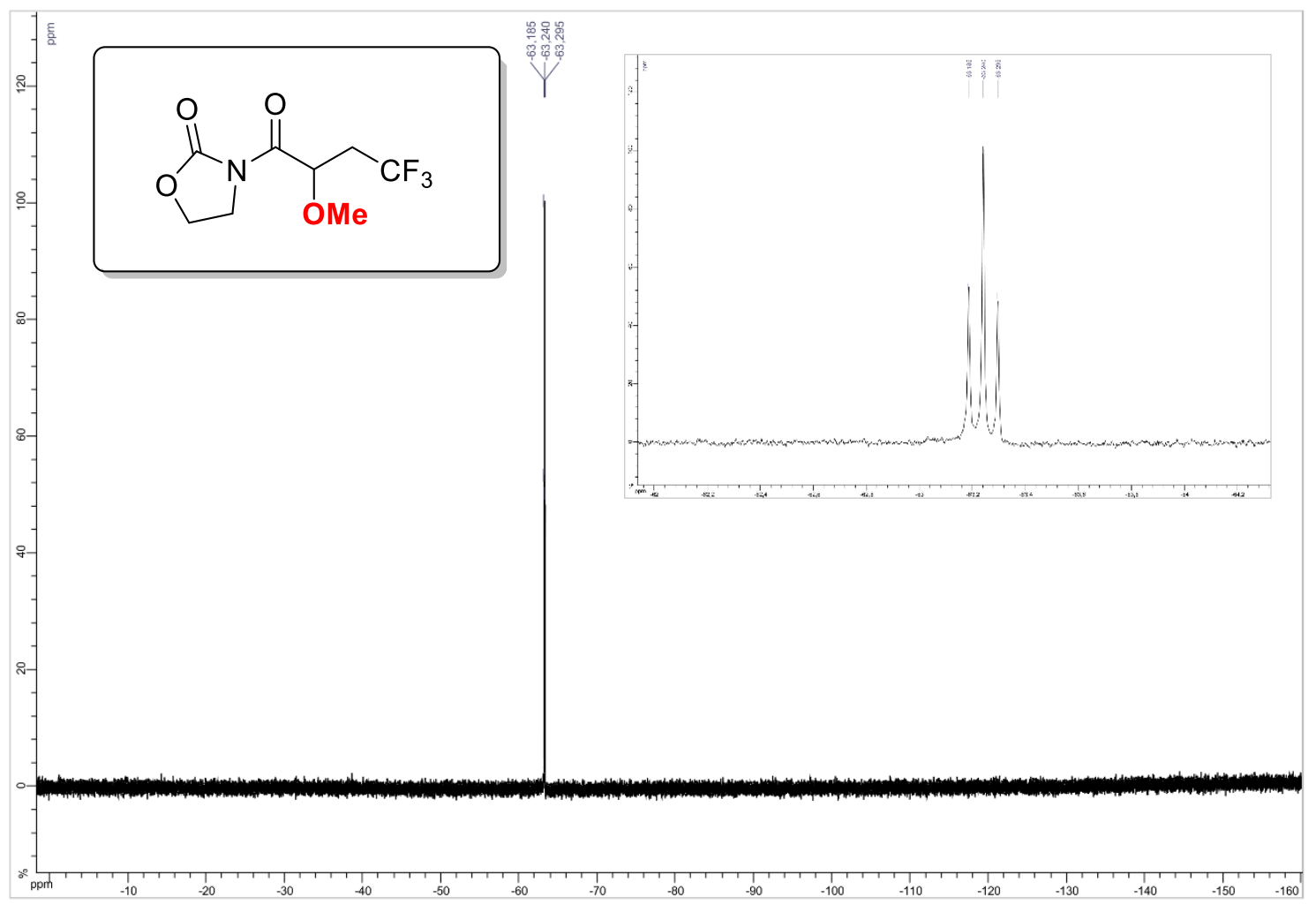


3-(2-Methoxyundec-10-enoyl)oxazolidin-2-one 7e

${ }^{1}$ H NMR (300 MHz, CDCl 3 )

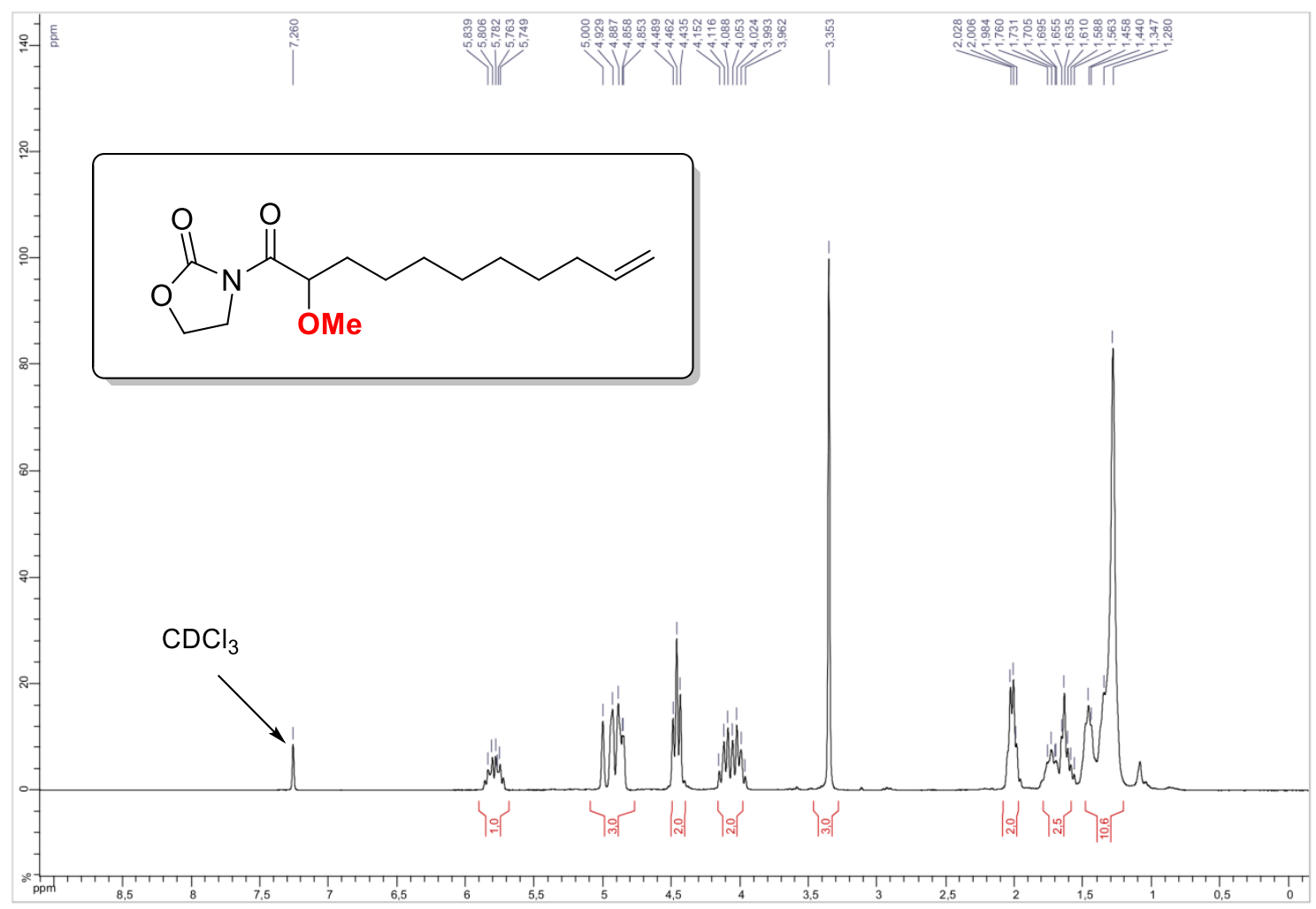

${ }^{13} \mathrm{C}$ NMR (75 MHz, CDCl3)

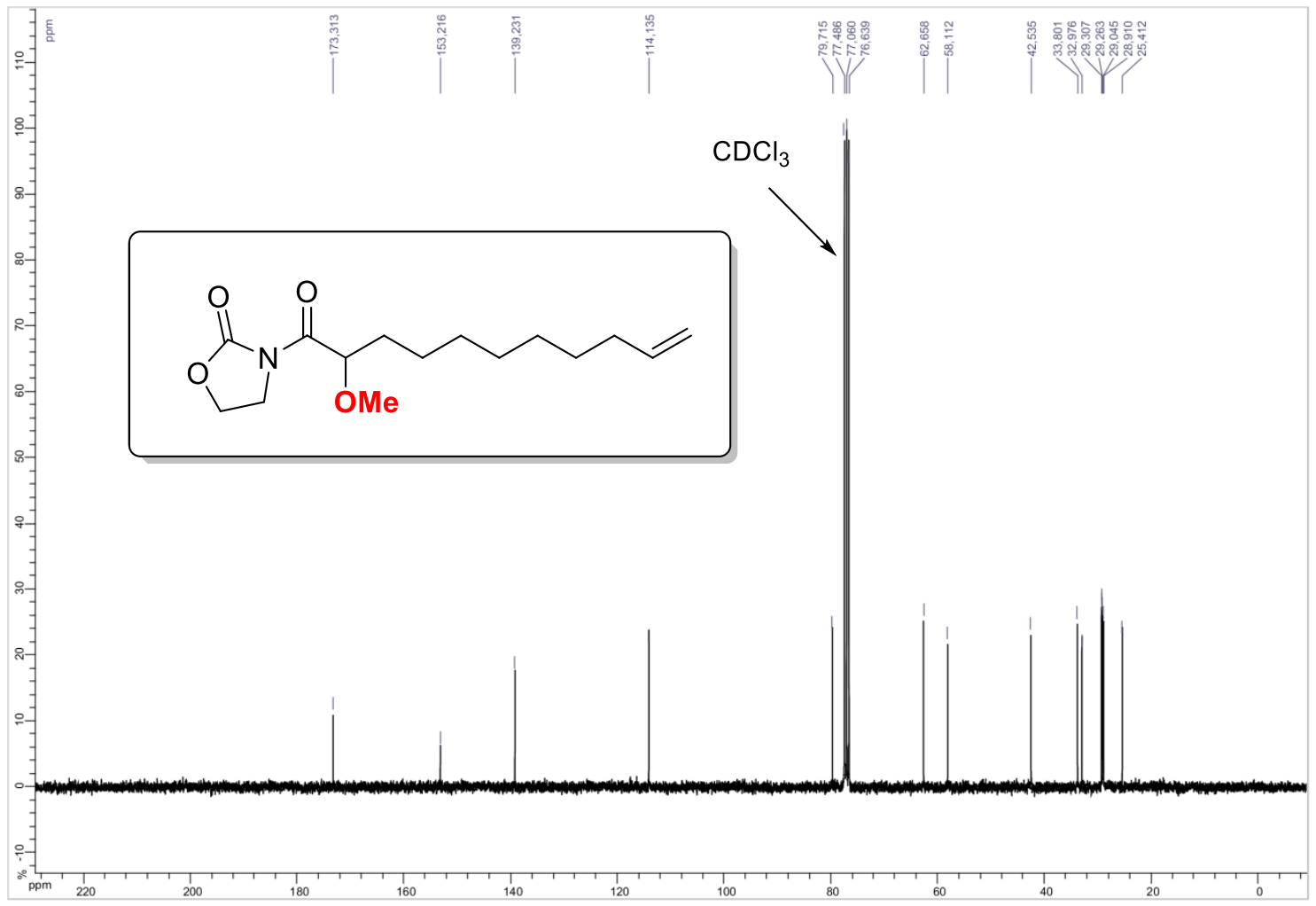


3-(2-Cyclohexyl-2-methoxyacetyl)oxazolidin-2-one 7f

${ }^{1} \mathrm{H}$ NMR (300 MHz, $\left.\mathrm{CDCl}_{3}\right)$

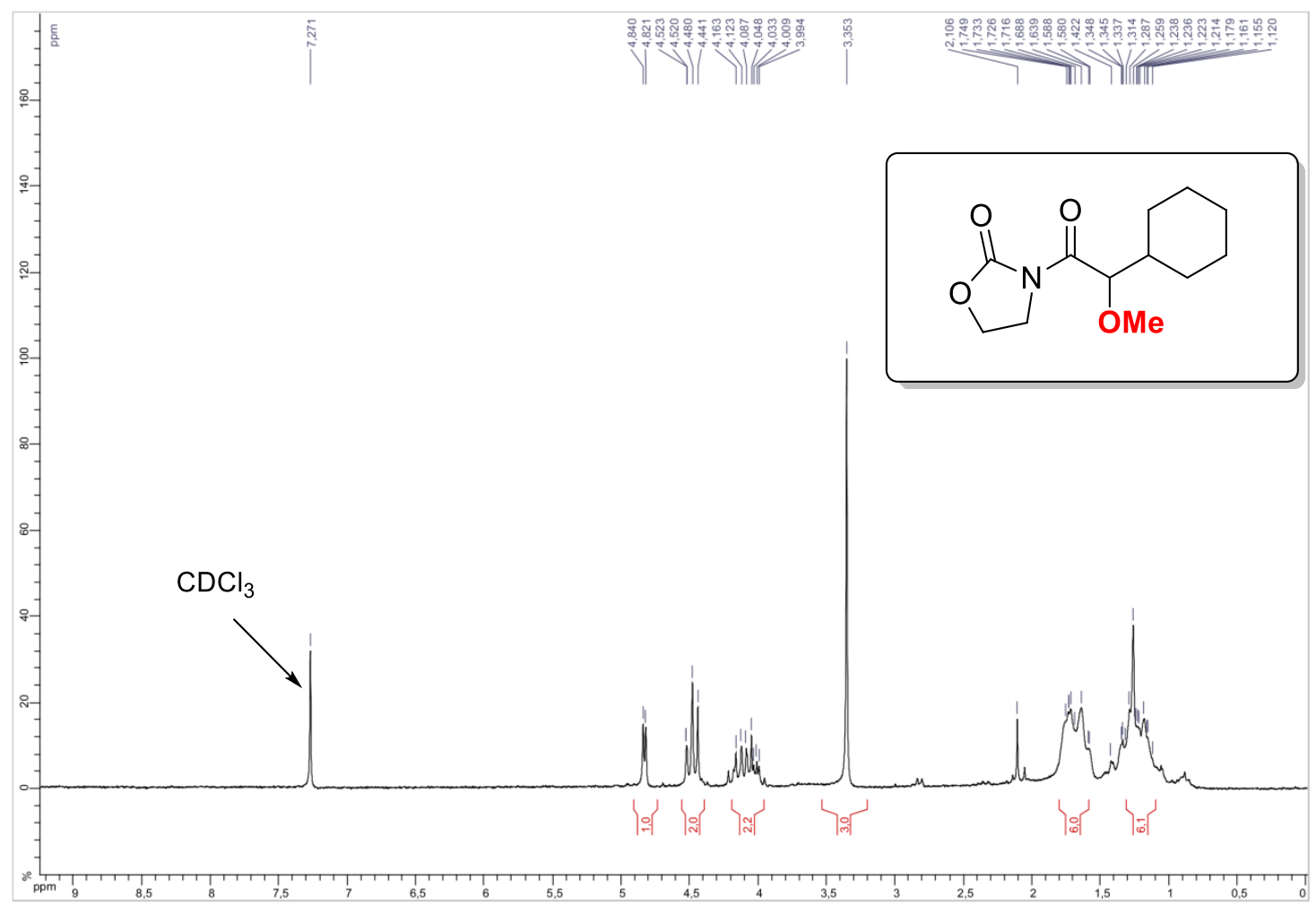

${ }^{13} \mathrm{C}$ NMR (75 MHz, $\left.\mathrm{CDCl}_{3}\right)$

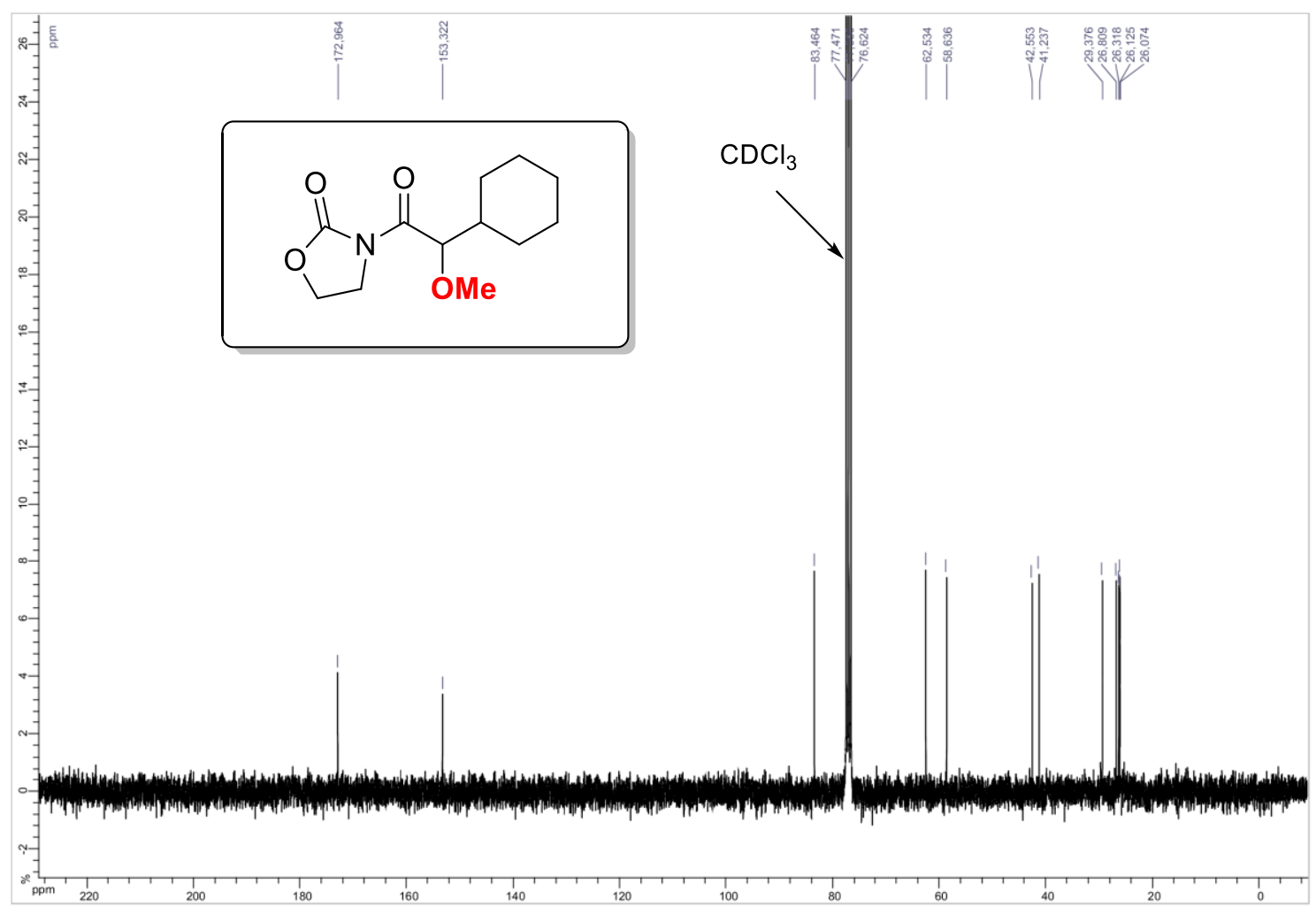


5-((3,5-dimethylphenoxy)methyl)-3-(2-methoxyhexanoyl)oxazolidin-2-one 7g ${ }^{1}$ H NMR (300 MHz, CDCl3)

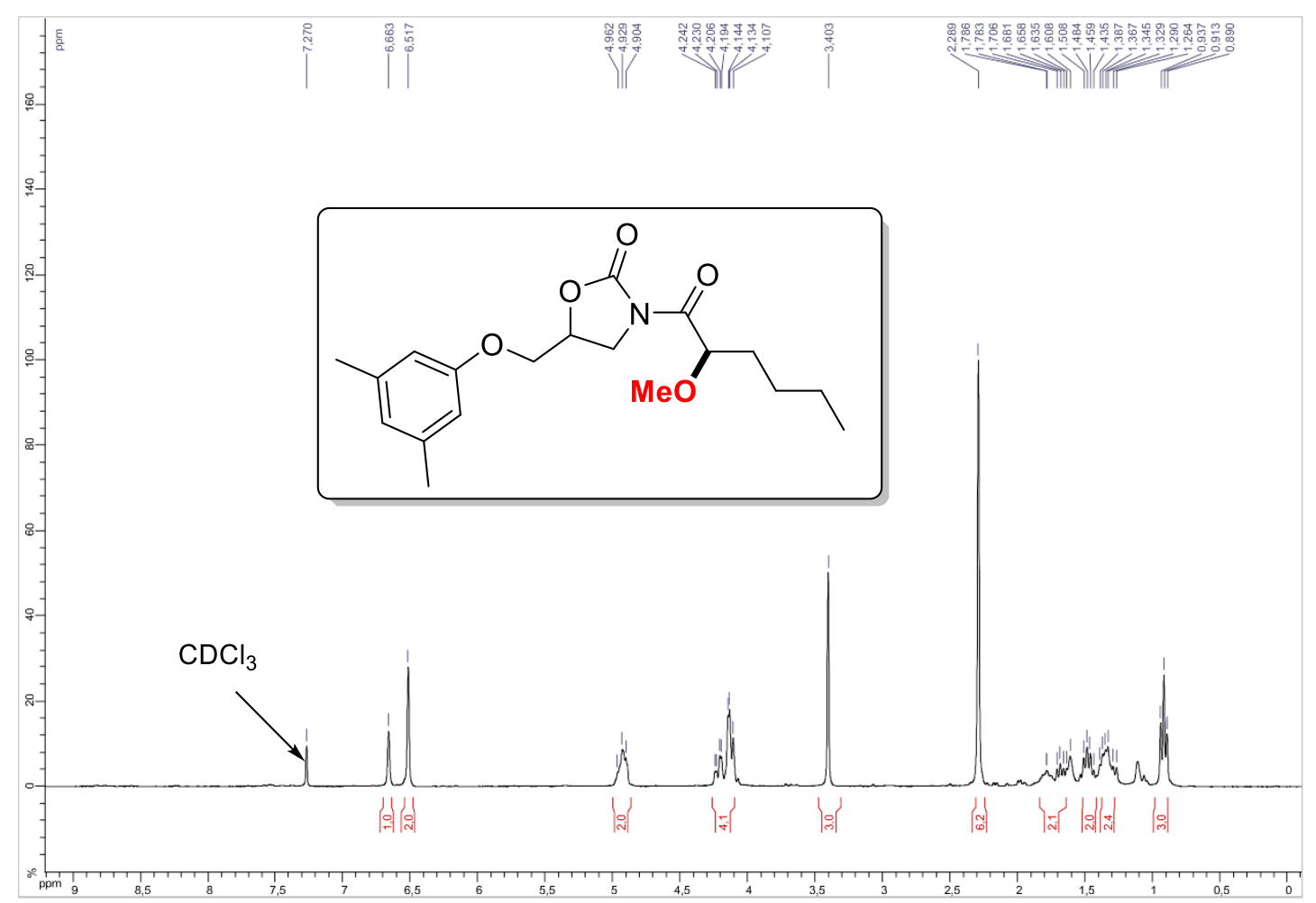

${ }^{13} \mathrm{C}$ NMR (75 MHz, CDCl3)

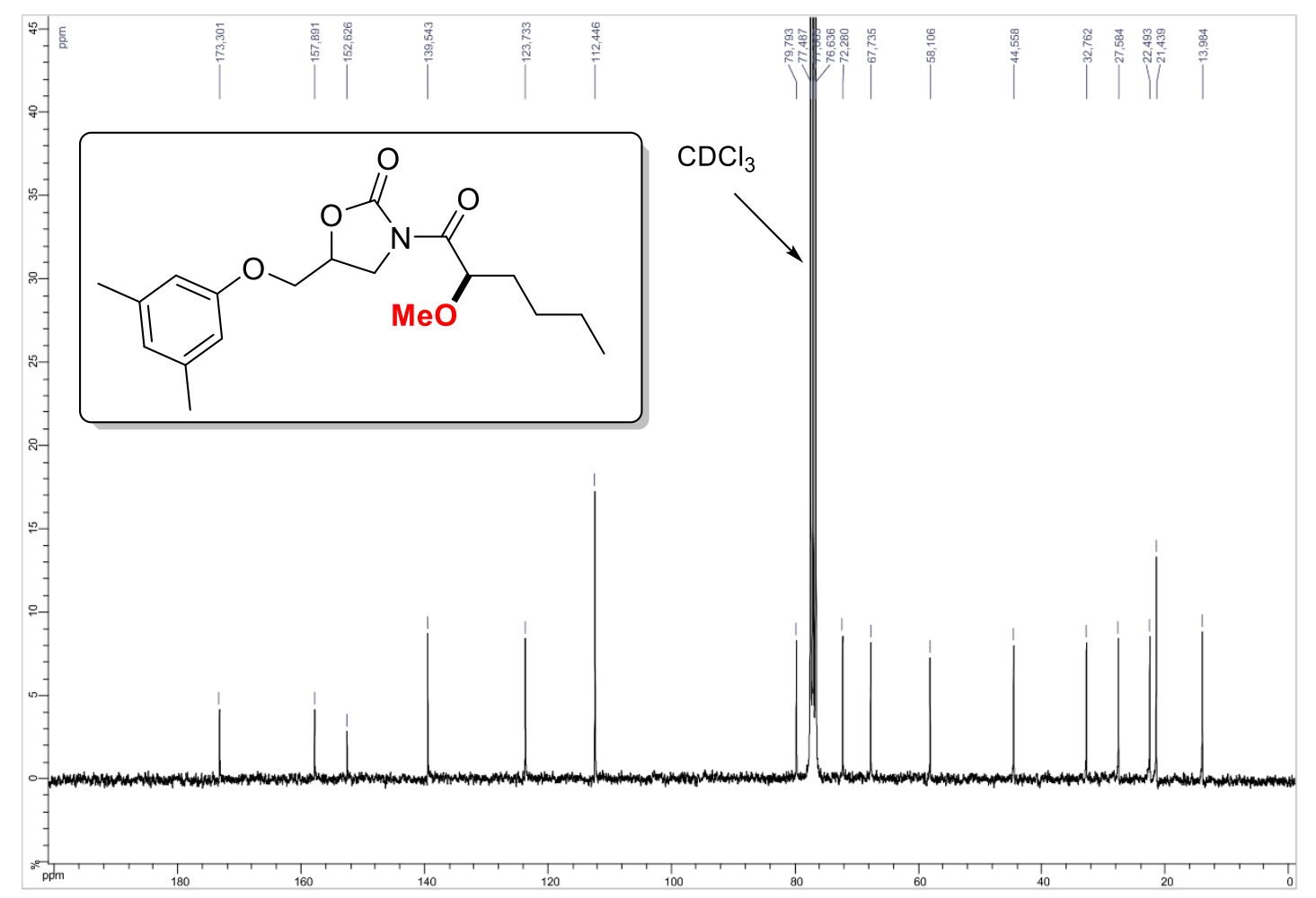


2,4,6-Trimethyl-1-((5-((triisopropylsilyl)oxy)pent-4-en-1-yl)oxy)pyridin-1-ium 4methylbenzenesulfonate 8

${ }^{1} \mathrm{H}$ NMR (300 MHz, CD3CN)

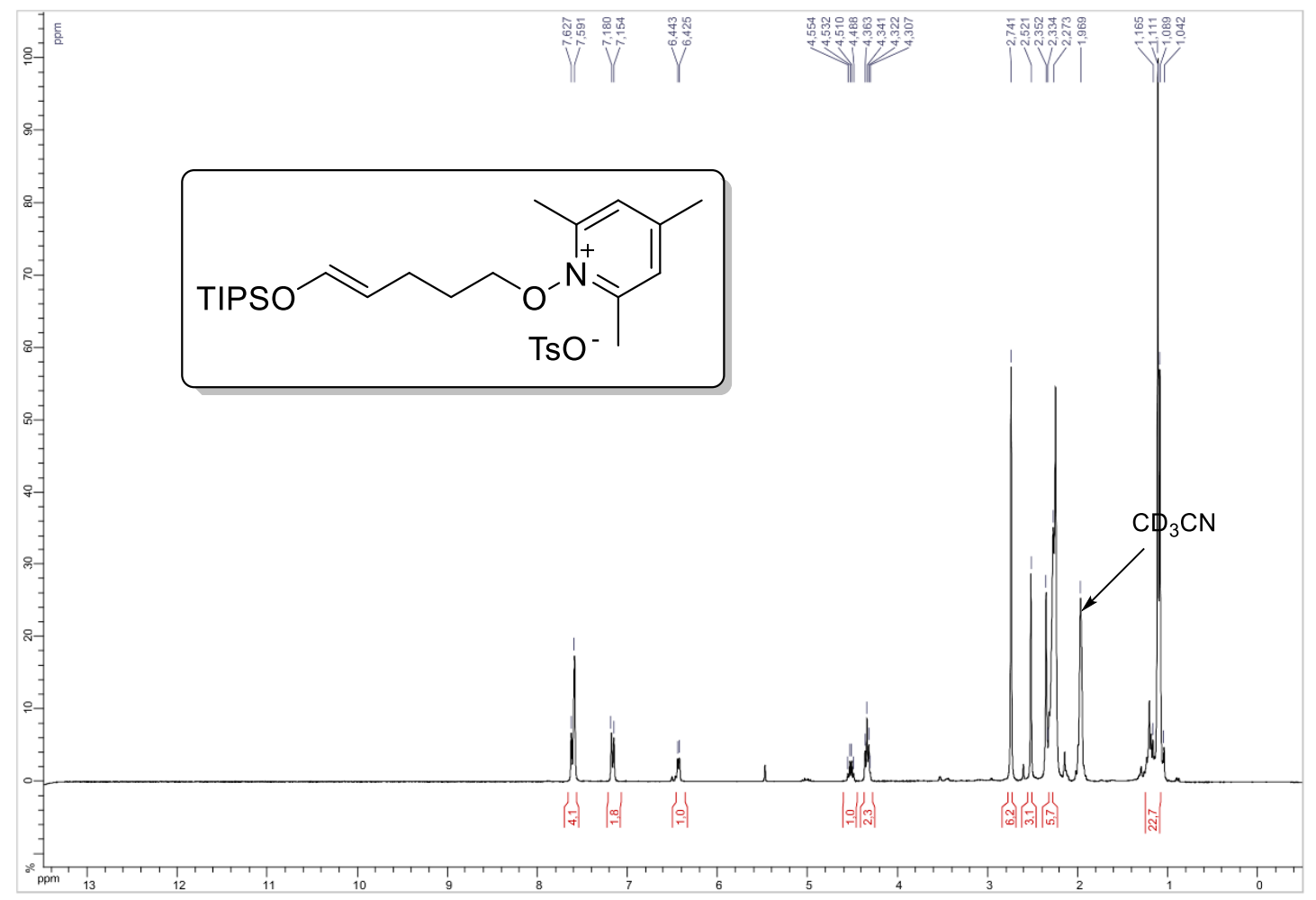

${ }^{13} \mathrm{C}$ NMR (75 MHz, CD3CN)

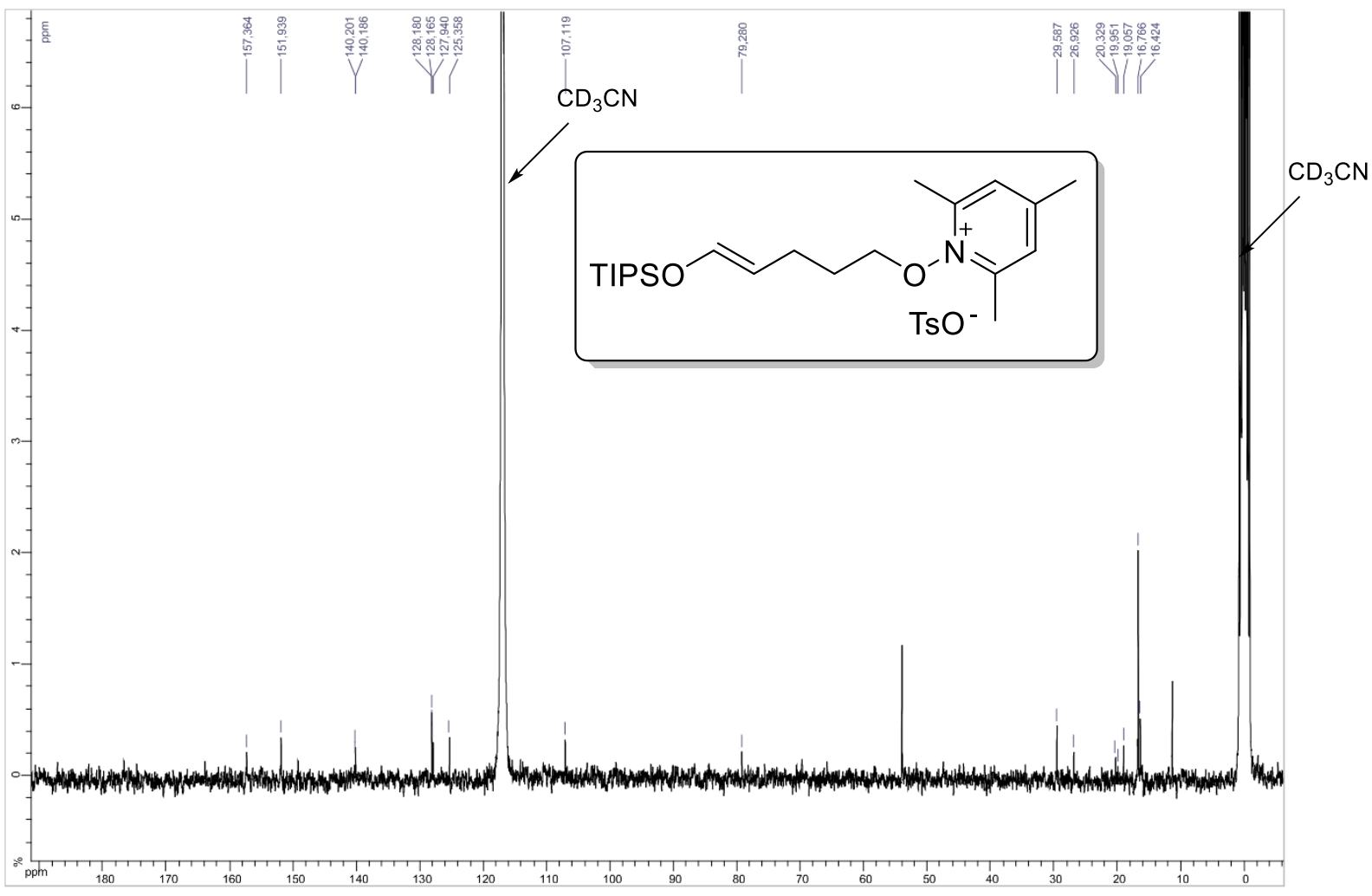


5-Hydroxypentyl 4-methylbenzenesulfonate 11

${ }^{1} \mathrm{H}$ NMR (200 MHz, CDCl 3$)$

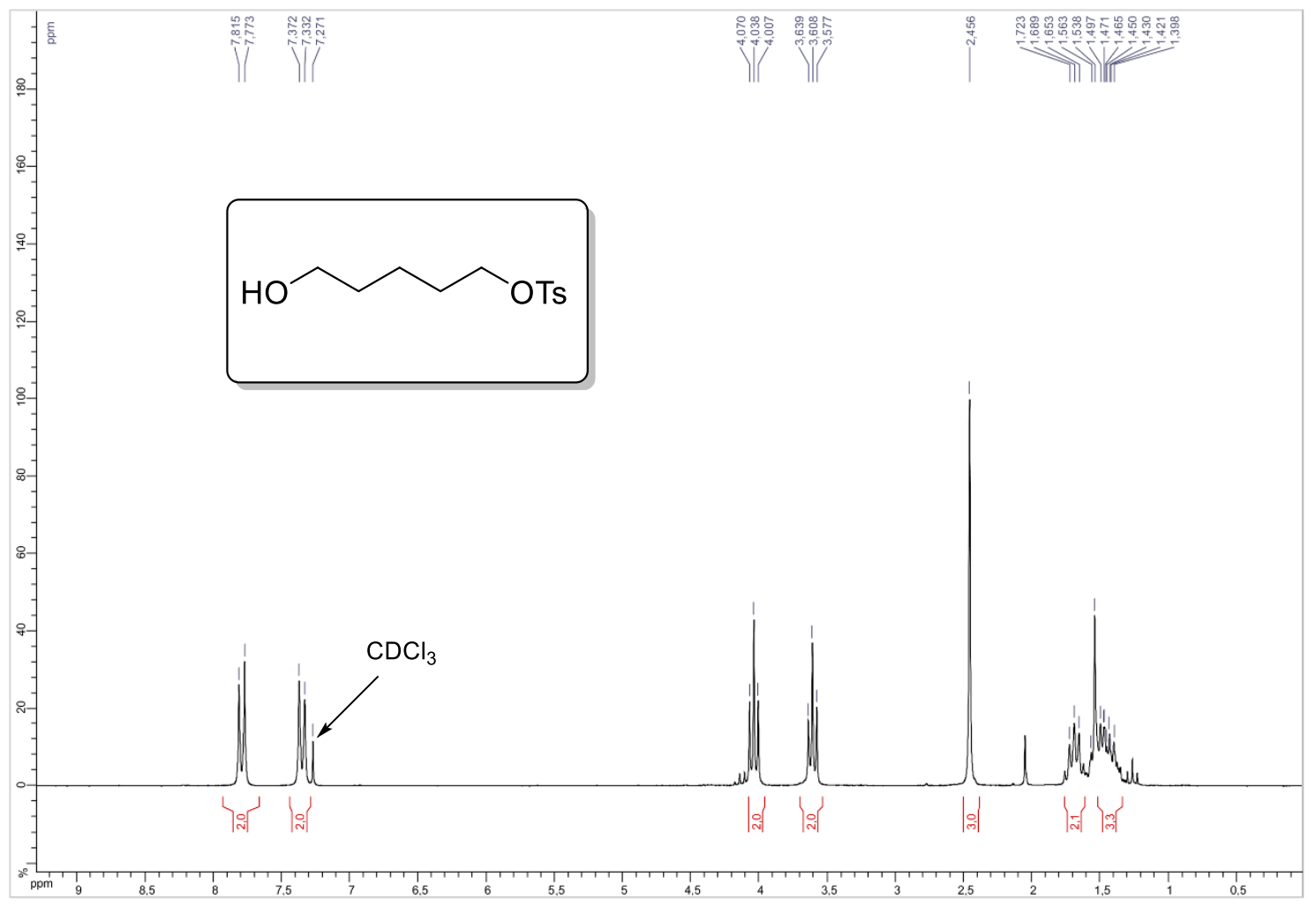


5-Oxopentyl 4-methylbenzenesulfonate 12

${ }^{1} \mathrm{H}$ NMR (200 MHz, CDCl 3$)$

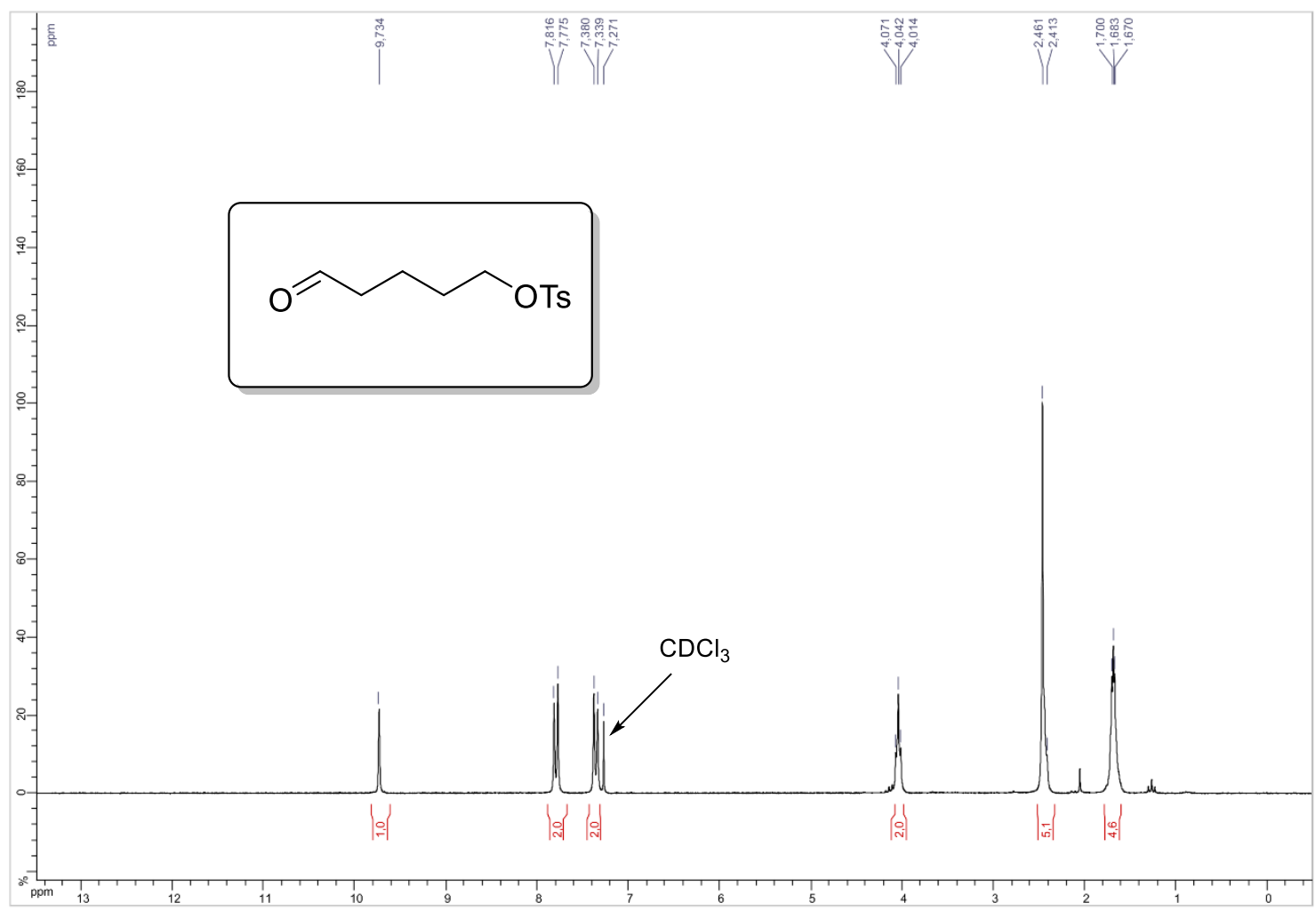


5-((Triisopropylsilyl)oxy)pent-4-en-1-yl 4-methylbenzenesulfonate 13

${ }^{1} \mathrm{H}$ NMR (300 MHz, CD3CN)

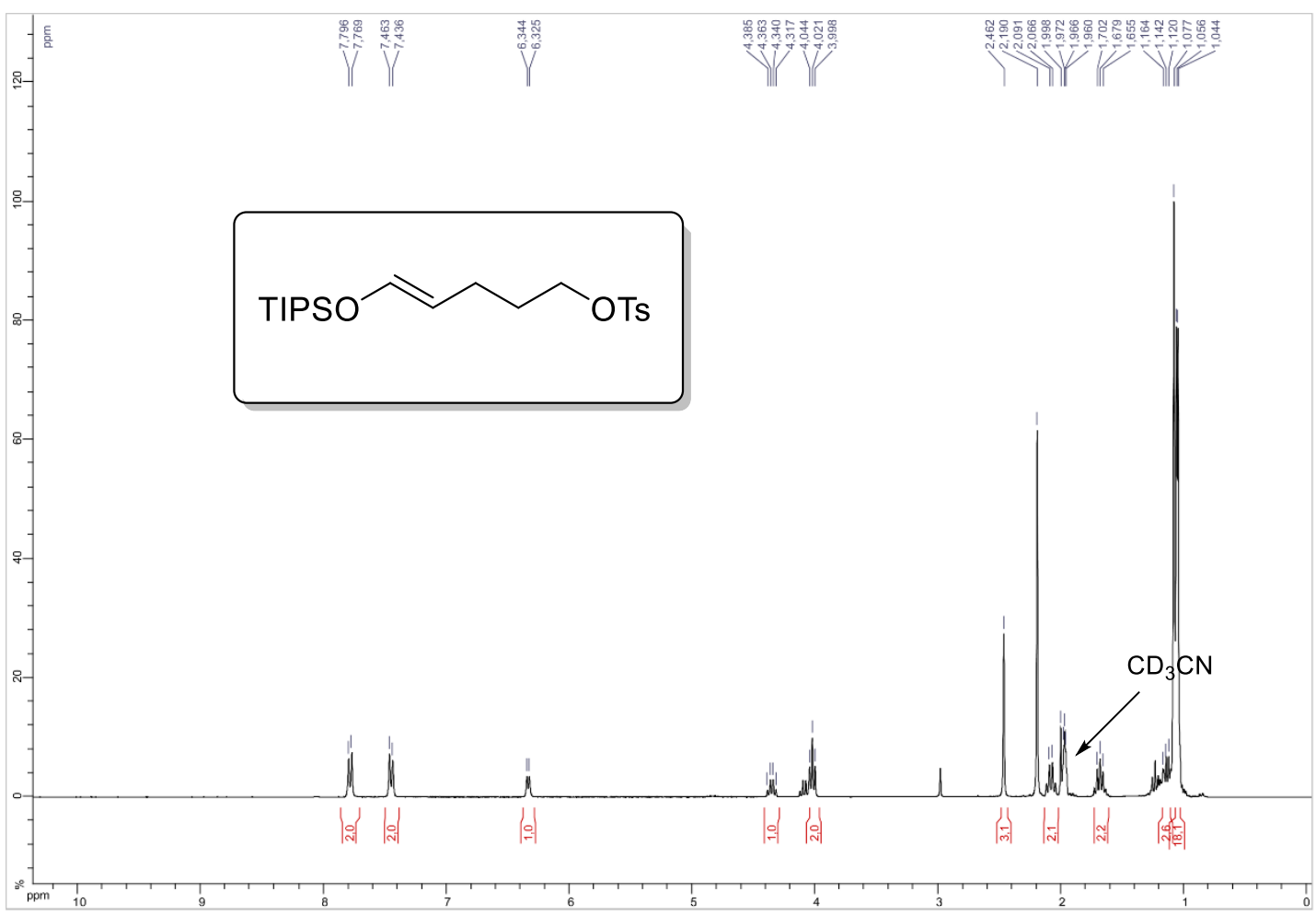

${ }^{13} \mathrm{C}$ NMR (75 MHz, CD3CN)

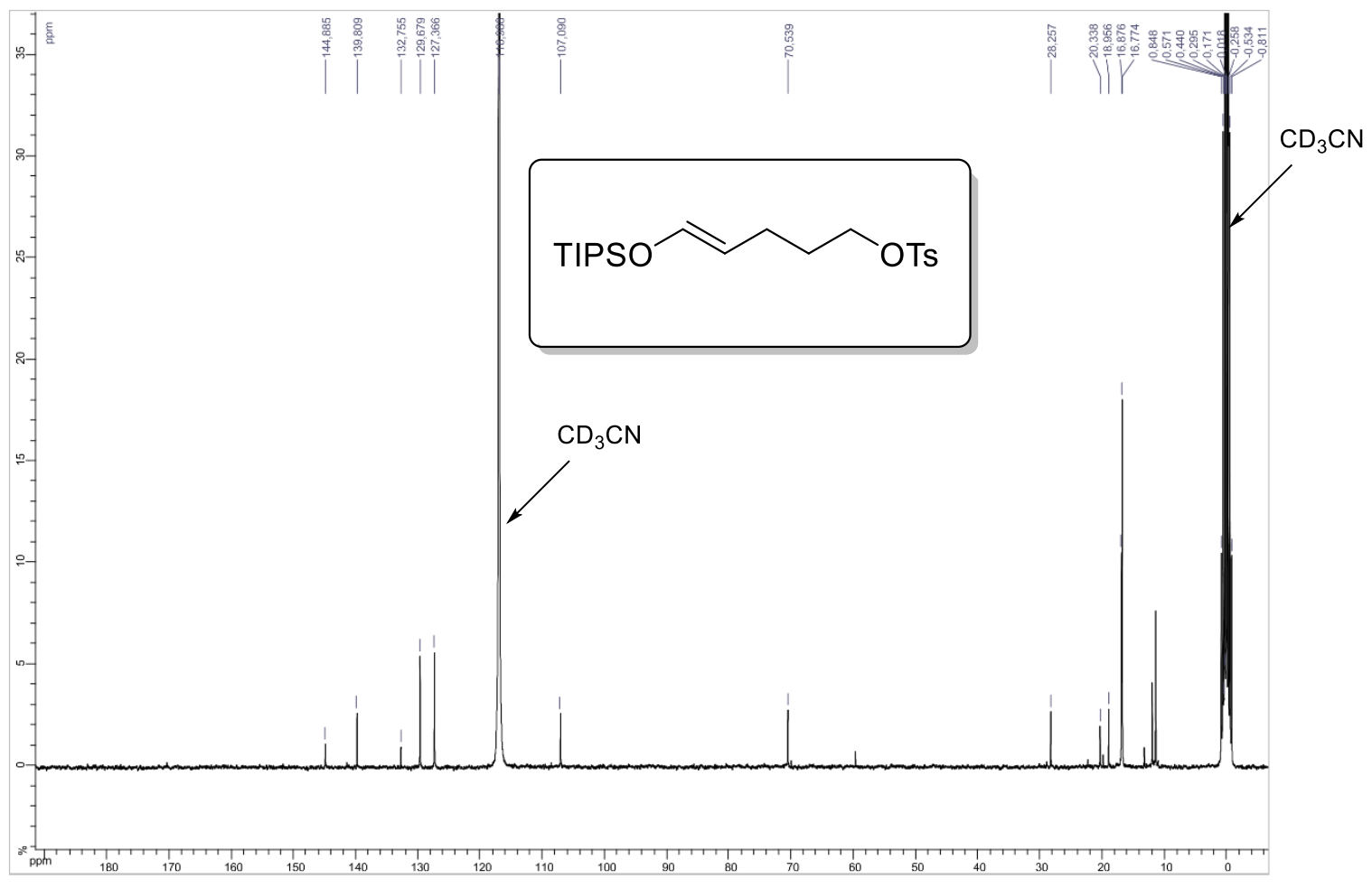


4-Methyl-4-(pyridin-4-yl)pentan-1-ol 14

${ }^{1}$ H NMR (200 MHz, acetone-d6)

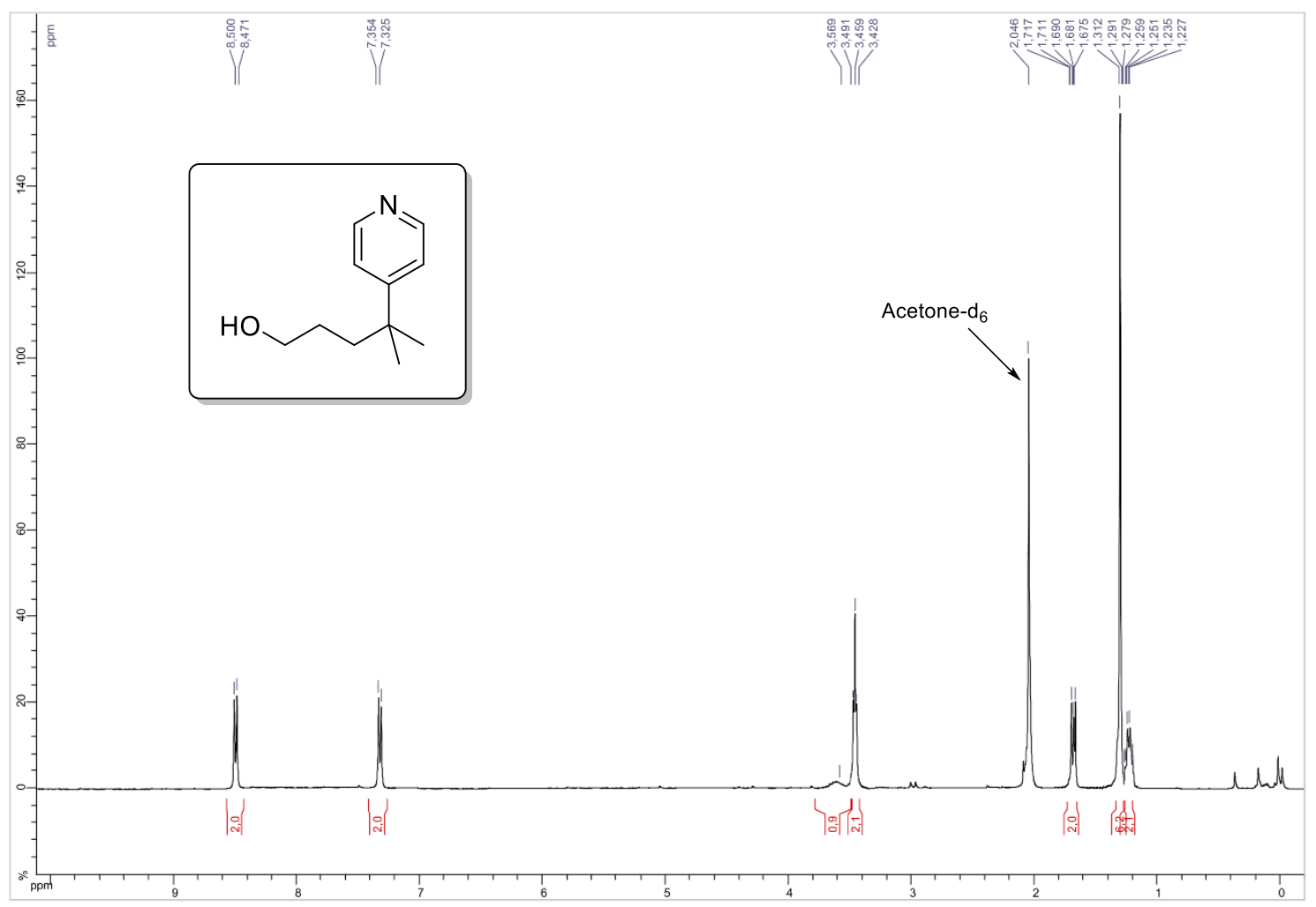




\section{References}

[1] Barthelemy, A.-L.; Tuccio, B.; Magnier, E.; Dagousset, G. Angew. Chem. Int. Ed. 2018, 57, 13790.

[2] a) de Nanteuil, F.; Waser, J. Angew. Chem. Int. Ed. 2011, 50, 12075. b) Chu, X.-Q.; Ge, D.; Wang, M.-L.; Rao, W.; Loh, T.-P.; Shen, Z.-L. Adv. Synth. Catal. 2019, 361, 4082. c) Sun, J.; Kozmin, S. A. Angew. Chem. Int. Ed. 2006, 45, 4991.

[3] Galante, E.; Okamura, T.; Sander, K.; Kikuchi, T.; Okada, M.; Zhang, M.-R.; Robson, M.; Badar, A.; Lythgoe, M.; Koepp, M.; Årstad, E. J. Med. Chem. 2014, 57, 3, 1023.

[4] Zhang, P.; Shen, H.; Zhu, L.; Cao, W.; Li, C. Org. Lett. 2018, 20, 22, 7062.

[5] Kobayashi, S.; Yokoi, T.; Inoue, T.; Hori, Y.; Saka, T.; Shimomura, T.; Masuyama, A. J. Org. Chem. 2016, 81, 1484.

[6] Kim, I.; Park, B.; Kang, G.; Kim, J.; Jung, H.; Lee, H.; Baik, M.; Hong, S. Angew. Chem. Int. Ed. 2018, 57, 15517.

[7] a) Yayla, H. G.; Peng, F.; Mangion, I. K.; McLaughlin, M.; Campeau, L.-C.; Davies, I. W.; DiRocco, D. A.; Knowles, R. R. Chem. Sci. 2016, 7, 2066; b) Ji, Y; DiRocco, D. A.; Hong, C. M.; Wismer, M. K.; Reibarkh, M. Org. Lett. 2018, 20, 2156.

[8] Hatchard, C. G.; Parker, C. A. Proc. R. Soc. London, Ser. A 1956, 235, 518.

[9] Juris, A.; Balzani, V.; Belser, P.; Vonzelewsky, A. Helv. Chim. Acta 1981, 64, 2175. 